DEPARTMENT OF THE INTERIOR

UNITED STATES GEOLOGICAL SURVEY

GEORGE OTIS SMITH, DIRECTOR

BULLETIN 422

\title{
THE ANALYSIS OF
}

\section{SILICATE AND CARBONATE ROCKS}

A REVISION OF BULLETIN 305

BY

W. F. HILLEBRAND

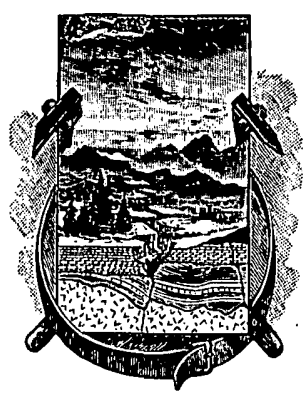

WASHINGTON

GOVERNMENT PRINTING OFFICE

1910 



\section{CONTENTS.}

Introduction . Page.

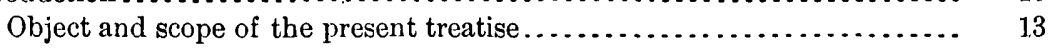

Acknowledgments........................................... 15

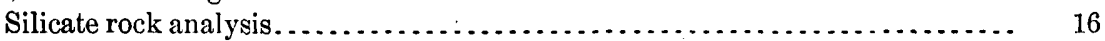

Part I. Introduction....................................... 16

1. Importance of complete and thorough analyses ............... 16

2. The constituents occurring in silicate rocks ................. 22

3. The distribution and occurrence of certain minor constituents.... 23

4. Summation of analy tical results and limits of allowable error ..... 27

5. Statement of analyses............................... 29

6. Time needed for making an analysis .................... 30

7. Useful appliances and apparatus ........................ 30

A. Crucible tongs................................ 30

B. Radiators for volatilizing liquids and solids.............. 31

C. Perforated disk for crucibles........................ 32

D. Weighing scoop or trough .......................... 33

E. Colorimeters:.................................... 33

a. First Survey form ............................. 33

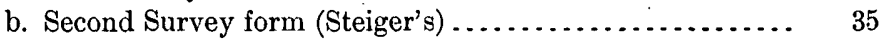

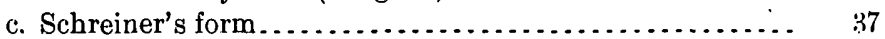

8. Preliminary qualitative analysis $\ldots \ldots \ldots \ldots \ldots \ldots \ldots \ldots \ldots . . \ldots \ldots$

9. Quality of reagents..................................... 39

Part II. Methods applicable to silicate rocks ..................... 41

1. Introductory remarks................................ 41

2. Specific gravity (porosity) .............................. 43

General remarks ................................... 43

A. By suspension in water............................ 43

a. Ordinary method for specific gravity ................ 43

b. Method for specific gravity and porosity combined...... 45

c. Penfield's method for mineral fragments............. 46

B. Pycnometer method .............................. 47

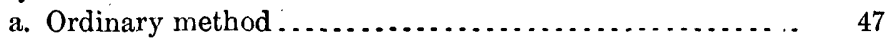

b. Refined method ................................ 48

C. Heavy solutions not suitable for rocks.............. 50

3. Preparation of sample for analysis ...................... 50

A. Quantity of rock to be crushed.................... $\quad 50$

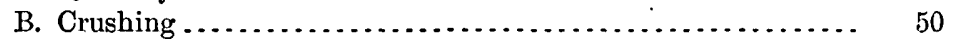

a. Survey methods .............................. 50

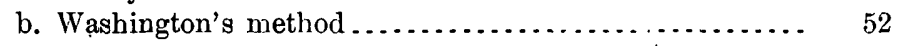

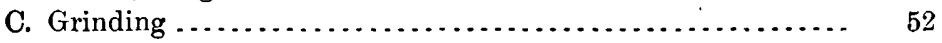

D. Abrasion of mortar and pestle...................... 56

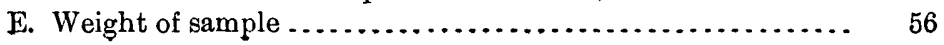


Silicate rock analysis-Continued.

Part II. Methods applicable to silicate rocks-Continued.

Page.

C. Importance of employing air-dry powder for analysis........

D. Argument in favor of including hygroscopic water in the

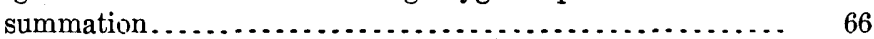

5. Water.-Methods of determination........................ 67

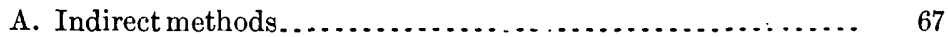

a. General considerations......................... 67

b. Methods involving the use of dehydrating agents :..... 67

c. Methods involving application of heat.............. 69

$\alpha$. Fractional determinations..................... 69

$\beta$. "Loss on ignition" method for total water......... 71

B. Direct methods without absorption tubes-Penfield's methods. $\quad 72$.

a. For minerals easily deprived of their water .......... $\quad 72$

b. For minerals not easily deprived of their water........ 73

C. Direct methods with absorption tubes. .............. 73

a. General considerations........................... 73

b. Penfield's procedure........................... 74

c. Steiger's application of the toluene oven............ 75

d. Chatard's oven for temperatures up to $300^{\circ}$ and over.... 76

e. Gooch's apparatus......................... 78

f. Merits of the above forms of apparatus............. 82

g. Jannasch's methods........................... 82

6. Silica, separation from alumina, etc.................... 83

A. Alternative methods of decomposing the rock.......... 83

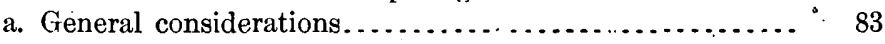

b. Decomposition of refractory silicates by hydrochloric acid under pressure................................ 85

c. The boric-oxide method of Jannasch and Heidenreich... 86

$c$. Preparation of the boric oxide. . . . . . . . . . . . . 86

$\beta$. Treatment of easily decomposable silicates......... 86

$\gamma$. Treatment of refractory silicates................ 86

$\delta$. Further treatment after fusion................. 87

$\varepsilon$. Possible objections to the boric-oxide method....... 87

d. The sodium-carbonate method................... 87

$\alpha$. Advantages of sodium carbonate over sodium potassium carbonate........................ 87

$\beta$. Purity of the sodium carbonate.............. 88

$\gamma$. The fusion with normal sodium carbonate........ 88

$\delta$. Fusion with sodium bicarbonate............. 90

$\varepsilon$. Treatment after fusion. ..................... 90

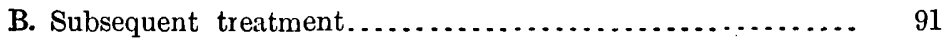

a. Separation of silica............................ 91

$\alpha$. Reasons for adoption of accepted procedure........ 91

$\beta$. Procedure in absence of notable amounts of fluorine. $\quad 92$

$\gamma$. Procedure with rocksand minerals containing fluorine. 94

b. Ignition of silica........................... 94

c. Correction for impurities in the silica. . . . . . . . . . . 95

d. Accuracy of the silica determination ............... 96

e. Composition of the residue obtained from the silica..... 96

f. Platinum in filtrates. . . . . . . . . . . . . . . . . . . . . 97

7. Metal precipitable by hydrogen sulphide................ 98 
Silicate rock analysis-Continued.

Part II. Methods applicable to silicate rocks-Continued. Page.

8. Aluminum-total iron ................................ 98

A. Indirect method for aluminum (titanium) ............. 98

a. Preliminary remarks........................... 98

b. Precipitation of aluminum, iron, etc., without precipitating manganese........................... 99

$\alpha$. Precipitation by ammonia.................... 99

$\beta$. The basic-acetate precipitation.................. 100

c. Precipitation of 'iron, aluminum, etc., together with manganese ............................... 102

$\alpha$. By ammonia and ammonium persulphate........ 102

$\beta$. By ammonia and ammonium sulphide........... 103

d. Recovery of iron and aluminum from the filtrates...... 103

$\alpha$. After an ammonia precipitation................ 103

$\beta$. After a basic-acetate precipitation.............. 103

$\gamma$. After precipitation by ammonia and ammonium persulphate.............................. 104

e. Ignition of the precipitate of iron, aluminum, etc., 0xides.. 104

f. Recovery of silica and possible barium in the alumina precipitate.................................. 105

ж. Silica....................................... 105

$\beta$. Barium ................................... 107

B. Determination of iron in the precipitate of alumina, etc..... 107

a. Without regard to the presence of vanadium......... 107

$\alpha$. Reduction of the iron ...................... 107

$\beta$. Titration of the iron ........................ 108

$\gamma$. Further treatment of the solution after titration..... 109

b. Having regard to the presence of vanadium.......... 109

c. Determination of the true value for ferric iron.......... 110

C. Methods aiming at the more or less direct determination of aluminum .................................. 1111.

a. After first removing iron as sulphide............... 111

b. By extraction with a fixed caustic alkali............. 111

c. Methods of Dittrich............................. 112

d. Direct precipitation of aluminum by phenylhydrazine... 112

9. Manganese, nickel, cobalt, copper, and zinc............... 113

A. Difficulties in the way of a correct gravimetric determination of manganese................................. 113

B. Precipitation of the group and separation of its constituents. - 114

a. The ammonium-sulphide method................. 114

$\alpha$. Its advantages and disadvantages............... 114

$\beta$. Precipitation by ammonium sulphide and separation of manganese and zinc from nickel, cobalt, and copper.

b. Manganese and zinc.............................

c. Nickel, cobalt, copper........................... 116

C. Colorimetric determination of manganese ................ 116

a. Preliminary treatment........................ 116

b. Colorimetric determination of manganese by ammonium persulphate................................. 117

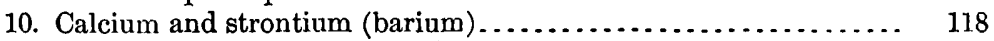

A. Separation from magnesium........................ 118

a. Precipitation and ignition of calcium and strontium oxialates together..............................

b. Notes on double precipitation, washing, and ignition of the oxalates 
Silicate rock analysis-Continued.

Part II. Methods applicable to silicate rocks-Continued.

10. Calcium and strontium (barium)-Continued.

B. Separation of strontium (barium) from calcium ............ 119

a. By ether-alcohol............................... 119

b. By amyl alcohol.............................. 120

C. Behavior of barium ................................. 120

D. Separation of barium from strontium .................. 120

E. Other methods of determining the weight of calcium ....... 121

General statement.................................. 121

a. Volumetric method............................ 122

b. By weighing as the carbonate ...................... 122

c. By weighing as the sulphate...................... 122

d. By weighing as the fluoride ....................... 123

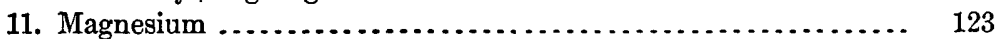

A. Precipitation ...................................... 123

a. Conditions necessary for the production of a normal precipitate....................................... 123

b. Precipitation ...................................... 124

$\alpha$. First precipitation............................ 124

$\beta$. Second precipitation......................... 125

B. Methods of collecting and igniting the precipitate .......... 125

a. Ordinary procedures........................... 125

b. Modification of Ulbricht........................ 126

C. Contamination by and removal of barium, calcium, and manganese......................................... 126

a. Barium .......................................... 126

b. Calcium ....................................... 127

$\alpha$. Preferred method ........................... 127

ß. Alternative method ........................... 127

c. Manganese ..................................... 127

D. Liability to error in the average magnesium determination... 128

12. Titanium .......................................... 128

A. Colorimetric determination with hydrogen peroxide........ 128

a. Principle of the method (Weller's) and the conditions that affect it........................................... 128

b. Preparation of the standard ....................... 130

c. The test solution ............................... 130

d. The color comparison ............................ 132

e. Correction for the color of ferric sulphate............ 133

$\alpha$. By allowing for the coloring power of the iron in the test solution ............................... 133

$\beta$. By addition of iron to the standard ............... 133

$\gamma$. By the use of phosphoric acid .................. 134

f. Correction for the effect of alkali sulphate............. 134

B. Gravimetric methods............................... 134

a. Gooch's method .................................. 134

$\alpha$. Description of the method .................... 134

$\beta$. Gooch's method not directly applicable to rocks containing zirconium .............................. 136

b. Methods of Dittrich and Freund................... 136

C. Superiority of the colorimetric and Gooch methods over the older ones ...................................... 138

13. Barium (zirconium, rare earths, total sulphur, chromium) ...... 138

A. The general method ............................. 138

B. The method without regard to zirconium and sulphur....... 140 
Silicate rock analysis-Continued.

Part II. Methods applicable to silicate rocks-Continued.

14. Zirconium ?

A. Author's method .................................... 141

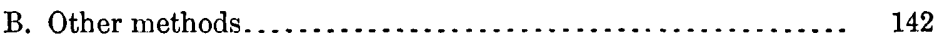

15. Rare-earth metals other than zirconium................... 143

A. Usual method ................................... 143

B. Alternative method ............................... 143

16. Phosphorus ......................................... 144

A. Procedure when material is ample .................. 144

a. Washington's method of preliminary treatment......... 144

b. Alternative method of preliminary treatment.......... 144

c. Subsequent treatment........................... 145

B. Procedure when material is scanty ................... 145

17. Chromium ......................................... 146

A. Gravimetric method ............................. 146

B. Colorimetric method.............................. 147

$\dot{\alpha}$. Preparation and strength of standard solution....... 147

$\beta$. Preparation of test solution................... 147

$\gamma$. Comparison of colors....................... 147

C. A few comparative data.......................... 148

18. Vanadium (chromium) and molybdenum ................ 148

A. Distribution of vanadium and molybdenum............. 148

B. Condition of vanadium in rocks .................... 149

C. Author's method.................................. 150

a. Description of the method....................... 150

b. Confirmatory qualitative tests.................... 151

c. Application of the method in presence of relatively much chromium ..................................... 152

D. Colorimetric method for vanadium .................... 154

19. Ferrous iron ......................................... 154

A. Oxidation of ferrous iron in minerals by grinding-preparation of sample.

B. Comparison of sealed-tube and hydrofluoric acid methodscomparative worthlessness of the former in rock analysis....

C. The modified Mitscherlich method.......................

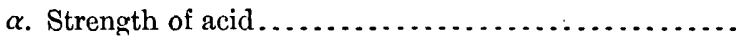

$\beta$. Filling, sealing, and heating of the tube............

$\gamma$. Reason for introducing gas and sealing as above directed ....................................

D. The hydrofluoric acid method....................... 161

a. Principle of the method ......................... 161

b. Defects of the method ......................... 162

$\alpha$. Oxidizability of divalent manganese by permanganate in presence of hydrofluoric acid............... 162

$\beta$. Extreme oxidizability of divalent iron by free oxygen in presence of bydrofluoric acid or a fluoride......

c. Influence of sulphides, vanadium, and carbonaceous matter on the determination of ferrous iron by the hydrofluoric acid method..............................

$\alpha$. Sulphides ................................. 165

ß. Vanadium ................................ 166

$\gamma$. Carbonaceous matter....................... 166 
Silicate rock analysis-Continued.

Part II. Methods applicable to silicate rocks-Continued.

19. Ferrous iron-Continued.

D. The hydrofluoric acid method-Continued. Page.

d. The method in its various modifications............... 167

$\alpha$. According to Pratt (modified) .................. 167

$\beta$. According to Cooke........................... 168

$\gamma$. According to Treadwell...................... 170

E. Uncertainties of the ferrous-iron determination.......... 171

20. Alkalies .......................................... 171

A. The J. Lawrence Șmith method..................... 171

a. Its advantages............................... 171

b. Reagents and apparatus......................... 171

c. Treatment of the mineral powder.................. 172

d. Separation of calcium and sulphuric acid.............. 173

e. Precipitation of potassium...................... 173

f. Sodium and lithium........................... 174

$\alpha$. Gooch's method for separating lithium .......... $\quad 175$

B. Preliminary treatment by other methods............... 176

a. The mercuric oxide method...................... 176

b. The ammonium carbonate method.................. 176

c. The amyl alcohol method ........................ 177

C. Direct separation of potassium by sodium cobaltinitrite..... 177

a. Preparation of the reagent..................... 177

b. Application of the method....................... 178

$\alpha$. Determination as chlorplatinate................ 178

$\beta$. Determination as perchlorate................... 178

21. Carbon dioxide, carbon............................... 179

A. Qualitative test for carbon dioxide.................. 179

B. Quantitative tests for carbon dioxide.................. 179

a. Direct method.................................. 179

b. Indirect method................................ 181

C. Carbon ........................................... 182

22. Chlorine ........................................ 182

A. Condition in rocks............................. 182

B. Determination of water-soluble chlorine............... 183

C. Determination of acid-soluble chlorine................ 183

a. By nitric acid................................ 183

b. By nitric and hydrofluoric acids................... 184

D. Determination of chlorine by alkali fusion............. 184

23. Fluorine (silica in presence of fluorine) .................. 184

A. Imperfection of the qualitative test for fluorine........... 184

B. Direct quantitative determination of fluorine............ 184

a. The method of Berzelius......................... 184

b. Testing of the calcium fluoride.................. 186

c. Accuracy of the method......................... 187

d. Determination of silica......................... 188

C. Indirect quantitative determination of fluorine.......... 188

a. Steiger's method............................... 188

b. Merwin's modification of Steiger's method............ 191

$\alpha$. Introductory remarks ......................... 191

$\beta$. Merwin's method........................... 192 
Silicate rock analysis-Continued.

Part II. Methods applicable to silicate rocks-Continued. Page.

24. Sulphur ........................................... 193

A. The determination of sulphur as barium sulphate......... 193

a. The errors involved ............................. 193

b. Avoidance or correction of errors................... 195

B. Determination of the condition of sulphur in rocks........ 197

C. Quantitative determination........................ 198

a. Total sulphur................................... 198

b. Fractional determinations ....................... 198

25. Boron .............................................. 199

26. Certain constituents in minute traces................... 201

27. The gases and vapors expelled by heat.................. 201

28. Special operations .................................... 203

A. Detection of nepheline in presence of olivine............. 203

B. Determination of soluble silica...................... 203

Carbonate rock analysis........................................... 205

Part I. Introduction...................................... 205

29. Qualitative comparison of carbonate and silicate rocks........ 205

30. Mineral composition of carbonate rocks.................. 207

31. Behavior toward different reagents as a means of distinguishing different carbonates.................................. 209

a. Differing solubility in tartaric and citric acids and in acid potassium sulphate ............................. 210

b. The Meigen reaction for distinguishing calcite from aragonite................................. 210

c. Distinguishing between calcite and dolomite ........... 211

Part II. Refined methods of analysis......................... 212

32. Silica, its separation from alumina, etc..................... 212

A. Methods of decomposing the rock .................. 212

a. When the inorganic residue is to be separately analyzed.. 212

b. When the inorganic residue is not to be separately analyzed. 213

$\alpha$. By solution in acid after strong ignition........... 213

$\beta$. By solution in acid after heating with sodium carbonate................................. 214

B. Separation of silica ............................... 214

33. Aluminum, total iron, titanium (silica, manganese); precipitation

in company with phosphorus, etc..................... 215

A. Precipitation of aluminum, iron, etc.................. 216

a. Collective precipitation of aluminum, iron, manganese, titanium, and phosphorus by ammonium sulphide.....

b. Precipitation of aluminum, iron, titanium, and phosphorus by ammonia.............................

c. Precipitation of aluminum, iron, titanium, and phosphorus by the basic-acetate method................... 217

B. Treatment of the filtrates from alumina, etc............. 217

C. Solution and separation of the oxides obtained in A and B.... 218

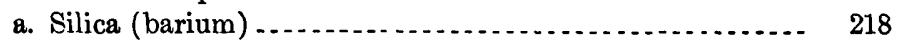

b. Iron .................. 218

c. Titanium ........... 219

d. Aluminum........... 219 
Carbonate rock analysis-Continued.

Part II. Refined methods of analysis-Continued. . . Page.

34. Manganese ....................................... 219

A. In the filtrates from 33. B.......................... 219

B. Separate determination of manganese............... 220

35. Copper, nickel, cobalt, lead, zinc, rare earths, chromium, vainadium, molybdenum............................. 220

36. Calcium, strontium, barium, magnesium (manganese)........ 221

37. Phosphorus ..................................... 222

38. Ferrous iron ......................................... 222

A. In the absence of carbonaceous matter................ 222

a. Ferrous iron soluble in sulphuric acid............... $\quad 222$

b. Ferrous iron in the insoluble residue................ 223

c. Total ferrous iron................................ 223

B. In presence of carbonaceous matter.................. 223

39. Alkalies .......................................... 224

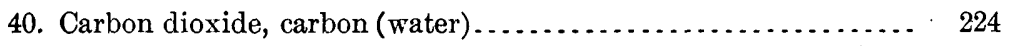

A. Determination of carbon dioxide.................... 224

B. Determination of carbon of carbonaceous matter.......... 224

C. Simultaneous determination of water and of total carbon in both forms ........................................ 225

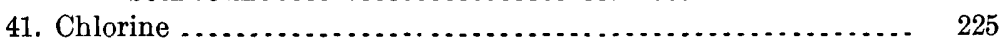

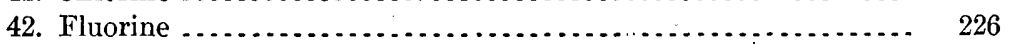

43. Sulphur................................................ 226

A. Tests as to its condition; determination of sulphate sulphur.. 226

B. Determination of total sulphur..................... 226

a. Methods of converting sulphides to sulphates........... 226

$\alpha$. By ignition without flux..................... 226

$\beta$. By ignition with sodium carbonate.............. 227

b. Treatment after ignition......................... 227

44. Water ........................................... 227

A. Hygroscopic water ................................ 227

B. Combined water.................................... 228

Part III. Condensed analysis................................ 228

45. Decomposition and solution ........................ 228

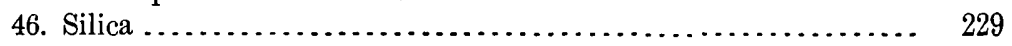

47. Aluminum, iron, etc ............................... 229

48. Calcium ............................................ 230

49. Magnesium .................................... 230

50. Alkalies............................................... 231

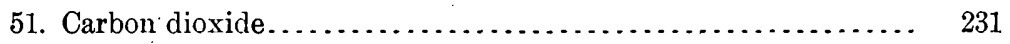

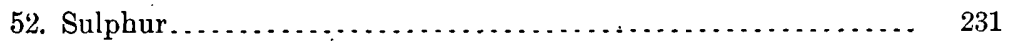

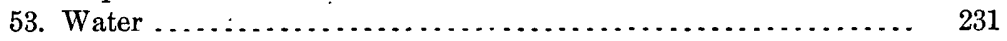

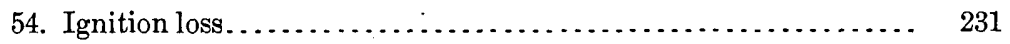

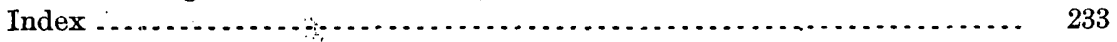




\section{ILLUSTRATIONS.}

1. Page.

FIG. 1. Platinum-tipped crucible tongs. . . . . . . . . . . . . . . . 30

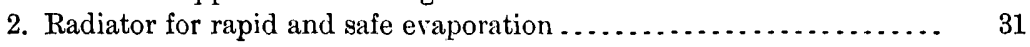

3. Device for excludoing flame gases from crucibles during ignition..... $\quad 32$

4. Weighing trough of platinum . ......................... 33

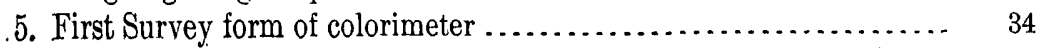

6. Second Survey form of colorimeter (Steiger's) $\ldots \ldots \ldots \ldots \ldots \ldots \ldots \ldots . \ldots \ldots$

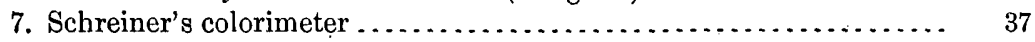

8. Moore's device to remove air from mineral powders............. 49

9. Ellis's steel mortar .................................... 51

10. McKenna ore grinder............................. 55

11. Penfield's tubes for water determination $\ldots \ldots \ldots \ldots \ldots \ldots \ldots \ldots \ldots \ldots . \ldots 2$

12. Penfield's oven for water determination $\ldots \ldots \ldots \ldots \ldots \ldots \ldots \ldots \ldots \ldots . \ldots . \ldots \ldots$

13. Penfield's tube for water determination $\ldots \ldots \ldots \ldots \ldots \ldots \ldots \ldots \ldots \ldots$

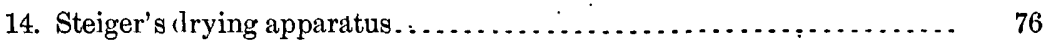

15. Chatard's drying oven for water determination $\ldots \ldots \ldots \ldots \ldots \ldots \ldots \ldots . \quad 77$

16. Modified form of Gooch tubulated platinum crucible for water determination ............................................ 78

17. Arrangement of Gooch apparatus during drying ............. 80

18. Arrangement of Gooch apparatus during fusion $\ldots \ldots \ldots \ldots \ldots \ldots \ldots \ldots . \quad 81$

19. Glass tube for determination of water (Jannasch) . . . . . . . . . . $\quad 82$

20. Glass tube for determination of water in special cases (Jannasch).... 83

21. Cooke's apparatus for ferrous-iron determination $\ldots \ldots \ldots \ldots \ldots \ldots \ldots . . . \ldots 9$

22. Treadwell's apparatus for ferrous-iron determination $\ldots \ldots \ldots \ldots \ldots \ldots \ldots$

23. The J. Lawrence Smith crucible for alkali determinations......... 172

24. Apparatus for carbon dioxide determinations. . . . . . . . . . . . . . . 180

25. Kreider's apparatus for indirect determination of volatile substances.. $\quad 181$

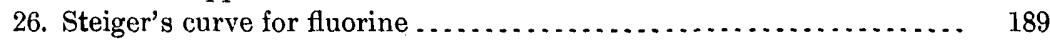

27. Merwin's diagrams for fluorine. ............................ 191 



\title{
THE ANALYSIS OF SILICATE AND CARBONATE ROCKS.
}

\author{
By W. F: Hillebrand.
}

\section{INTRODUCTION.}

\section{OBJECT AND SCOPE OF THE PRESENT TREATISE.}

The following paragraphs are reprinted from Bulletin 305, of which the present volume is a revision.

The literature relating to analysis of silicates is extensive but scattered, and in no single paper up to the time of the publication of Bulletin No. 148 of this Survey was there to be found, in the light of modern methods, a satisfactory exposition of the procedures to be followed or the precautions to be observed, especially in the search for some of the rarer constituents or those which, without being rare, have been of late years recognized as occurring persistently in small amounts. It was not the intention to make the chapter on rock analysis in that bulletin a manual on mineral or even rock analysis, but to show primarily the principles and methods by which. the major part of the analyses made up to that time in the laboratory of the Geological Survey had been conducted, and thus to afford a partial measure of the trustworthiness of those analyses. At the same time it was felt that the experience gained by the chemists of the Survey since the establishment of its first chemical laboratory in Denver might be useful to most chemists engaged in mineral and rock analysis. The favorable reception accorded it by chemists led to the republication of a portion of Bulletin No. 148 in more extended form as Bulletin No. 176. Since the publication of the latter bulletin in 1900 two other treatises on rock analysis have appeared: Manual of the Chemical Analysis of Rocks, by Dr. H. S. Washington (1904), and Anleitung zur Gesteinsanalyse, by Dr. Max Dittrich (1.905). The former is based largely on Bulletin No. 176; the latter, while adopting some of the methods advocated in that bulletin, adheres more to methods which are in greater favor in Germany than in this country. They both differ from Bulletin No. 176 in devoting less space to a discussion of principles and to the determination of the less common constituents of rocks. Doctor Washington's book enters into considerable detail in describing manipulations, since it was written for chemists, petrologists, mining engineers, and others who have not made a particular study of quantitative analysis. These books have not, therefore, rendered the Survey publication superfluous. On the contrary, since some methods have undergone improvement or been superseded by better ones, the time seems ripe for its republication in revised form.

Experience has shown that for the use of the student the outlines of some methods and procedures were too briefly sketched in the earlier publications. Where it seemed called for, therefore, these have received considerable elaboration. 
For the reasons that the chief carbonate rocks form so important an element in the composition of the earth's crust, and that the knowledge of their composition is of moment to the geologist as well as to the cement maker, who now makes enormous use of them, a special section devoted to their analysis has been added. The methods applied to their analysis differ in but unimportant respects from those used with the more siliceous rocks, since they are to a great extent themselves siliceous, contain essentially the same constituents, and therefore the same principles apply to both. Naturally they demand and receive briefer treatment.

The special problems often arising in the analysis of rocks of extraterrestrial originthe more or less stony meteorites-will not be considered here. An analysis of that kind should never be intrusted to the novice, but only to the chemist who has a knowledge of the composition and properties of the peculiar mineral constituents of those bodies and a judgment fit to cope with the oftentimes difficult problems presented by them.

The methods described in the following pages are not restricted altogether to those actually in use in the Survey laboratory. A number of alternative ones are given which are known or believed to be good, in order that those who may wish to use this treatise as a practical guide shall have some choice of selection in case the expensive apparatus or complicated arrangements sometimes preferred are not available. Where silicate and limestone analyses are very frequently made, however, it is a saving of time and money in the end to set up permanent arrangements for convenience in determining water, carbon, dioxide, ferrous iron, in making reductions in hydrogen, etc.

It was my intention to prepare a chapter on the mechanical separation in a small way of minerals by the various processes more or less in vogue, as panning, upward flow of water, heavy solutions, and magnetism, but lack of time, and in fact insufficient experience with some of the methods and solutions, have prevented its development. To those seeking information on some of these methods reference is made to the works of Rosenbusch-Wülfing, Mikroscopische Physiographie der Mineralien und Gesteine, volume 1 (1904); Rosenbusch-Iddings, Microscopical Physiography of Rock-making Minerals, fourth edition (1903); and Ferdinand Zirkel, Lehrbuch der Petrographie, second edition, volume 1 .

As the edition of Bulletin 305 approached exhaustion the question arose whether it should be reissued or revised. In view of certain discoveries that had been made since the appearance of that bulletin in 1907, which necessitated not only a rather radical change in the manner of preparing samples for analysis, but also modifications in some analytical procedures, the decision was made to revise it. This seemed all the more desirable, because a German translation of Bulletin 305 , with considerable new matter added by myself, had been prepared, and has since been published.

The following are the more important changes and additions in the present volume as compared with Bulletin 305. The preparation of the sample has been much modified in the endeavor to meet the need for one that shall not have taken up water from the air or have suffered oxidation with respect to ferrous iron or sulphides during grinding. The effect of fine grinding on the composition of the sample has necessitated changes in carrying out certain determinations, notably that for ferrous iron. The chapters on water have been rearranged, and that devoted to general considerations is now introduced by a section on the rôle of hydrogen in minerals. The 
chapter on the determination of titanium has been in part rewritten, in consequence of the discovery of the bleaching effect of alkali salts on the color produced by hydrogen peroxide. This discovery called also for an important addition to the chapter on fluorine. That on sulphur has been supplemented by a section on the errors involved in the determination of sulphur as barium sulphate and their avoidance or correction. Many other minor additions and alterations have been made.

The author's transfer in 1908 from the Geological Survey to the Bureau of Standards has made it impossible for him to keep as closely in touch with the literature of methods pertaining to or applicable to mineral analysis as he would have liked. This must serve both as excuse and apology if, as is probable, some new methods or modifications of methods that deserve recognition find no mention in the present volume.

\section{ACKNOWLEDGMENTS.}

To Drs. F. W. Clarke, E. T. Allen, E. C. Sullivan, R. C. Wells, A. L. Day, and F. K. Cameron, and Messrs. George Steiger and W. T. Schaller, my thanks are freely rendered for friendly criticism, advice, suggestions, and occasional direct assistance. 


\section{SILICATE ROCK ANALYSIS.}

\section{PART I.-INTRODUCTION.}

\section{IMPORTANCE OF COMPLETE AND THOROUGH ANALYSES.}

The composition of the ultimate ingredients of the earth's crustthe different mineral species. which are there found and of many of which its rocks are made up-was the favorite theme of the great workers in chemistry of the earlier half of the nineteenth century, and for the painstaking care and accuracy of Berzelius; Wöhler, and others the mineralogists and geologists of to-day have reason to be thankful. Considering the limited facilities at their disposal in the way of laboratory equipment and quality of reagents, the generul excellence of their work is little short of marvelous. As an outgrowtip. of and closely associated with the analysis of minerals came that the more or less complex mixtures of them-the rocks-to aid whos study by the petrographer and geologist a host of chemists have for many decades annually turned out hundreds of analyses of all grades of quality and completeness. With the growth and extraordinary development of the so-called organic chemistry inorganic chemistry gradually fell into a sort of disfavor. In many, even the best, European laboratories the course in mineral analysis, while maintained as a part of the curriculum of study, became but a prelude to the ever expanding study of the carbon compounds, which, multiplying rapidly and offering an easy and convenient field for original research and possible profit, proved more tempting to young chemists than the often worked over and apparently exhausted inorganic field. For one student devoting his time to higher research on inorganic lines fifty perhaps were engaged in erecting the present enormous structure of carbon chemistry. The instruction afforded the student in mineral analysis was confined to the ordinary separations of the commoner ingredients occurring in appreciable quantities, with little regard to supposed traces and with still less attempt to find out if the tabulated list really comprised all that the mineral or rock contained.

With the introduction of improved methods of examination by the petrographer, especially as applied to thin rock sections, and the use 
of heavy solutions, whereby, on the one hand, the qualitative mineral composition of a rock could be preliminarily ascertained with considerable certainty, and on the other chemical examination of the more or less perfectly separated ingredients was rendered possible, a great help and incentive was afforded to the few chemists engaged in rock. analysis. The microscope often obviated in part the necessity for tedious and time-wasting qualitative tests, and the heavy solutions, by permitting the concentration and separation of certain components, facilitated the detection of elements whose existence had long been overlooked.

Meanwhile, in the progress of chemistry new methods and reagents for qualitative detection and quantitative separation and estimation were gradually being devised and discovered. The belief that some well-established methods were adequate was shown to be unwarranted; :ome had to be discarded altogether; others were still utilizable after modification. In the light thus shed it became possible to explain many hitherto incomprehensible variations in the composition of some rock species or types, as shown in earlier analyses, and in not a few casps it appeared that the failure to report the presence of one or more c) nnents had obscured relations and differences which more thorough fminination showed to exist (see p. 19). Consequently there arose a "yling of distrust of much of the older work in the minds of those :emists and petrographers who were best fitted to judge of its probable qualities. This, and the incompleteness of nearly all the earlier work (and much of that of to-day, unfortunately), as shown by the largely increased list of those elements now known to enter into the normal composition of rocks, are rendering the old material less and less available to meet the increasing demands of the petrographer. ${ }^{a}$

And yet these demands on his part are, with few exceptions, by no means so exacting as they should be. Frequently the analysis is intrusted to a student without other experience than that gained by the analysis of two or three artificial salts and as many comparatively simple natural minerals, and with a laboratory instructor as adviser whose experience in rock analysis may be little superior to his own. In other words, one of the most difficult tasks in practical analysis is expected to be solved by a tyro, and his results are complacently accepted and published broadcast without question. Even to those thoroughly familiar with the subject rock analysis is a complex and often trying problem. Although long practice may have enabled

$a$ Dr. Fenry S. Washington, in Professional Papers Nos. 14 (Chemical analyses of igneous rocks published from 1884 to 1900 , with a critical discussion of the character and use of analyses) and 28 (The superior analyses of igneous rocks from Roth's Tabellen, 1869 to 1884, arranged according to the quantitative system of classification) of the United States Genlogical Surver, has done a most important work in sifting the great mass of dala accumulated in the thousauds of analyses published since 1869 . After b systematic critical examination he has assigned to each analysis a certain value on a scale of 5 . any of these values may be incorrect, but in most cases they undoubtedly give a fairly true measure the weight to be attached to a particular analysis.

20091-Bull. $422-10-2$ 
one to do certain parts of it almost mechanically, perplexing questions still arise which require trained judgment to properly meet and answer, and there is yet room for important work in some of the supposedly simplest quantitative determinations. If the results are to have any decided value for purposes of scientific interpretation and comparison, they must be the outcome of the work of one who is able to find his way through the intricacies of an analysis in which from fifteen to twenty-five components are to be separated and estimated with close approach to accuracy, and this a beginner can not hope to do. The conscientious chemist should have a live interest in this matter. $\mathrm{He}$ should work with a twofold purpose in view - that of lightening the labors of those who come after him by enabling them to use his work with less supplementary examination, and that of enhæncing his own reputation by meriting encomiums on work that has stood the test of time.

How little understood may be the principles underlying the treatment of bodies so complex and the accurate separation and determination of their constituents, even when these are comparatively few in number, has been strikingly shown during recent years in the work of several committees of chemists charged with the investigation of the methods employed in various lines of technical chemistry involving the analysis or assay of zinc ores, slags from the smelting of copper ores, argillaceous limestones, and cements. In all cases a most woful inability to obtain agreeing results is apparent; not only among those less experienced, but among those supposed to be most expert in each of the particular fields as well. Some improvement in silicate analysis has resulted from the investigations set on foot and the recommendations made by these committees, and further improvement may be expected, but the situation is yet anything but satisfactory. The art of analysis stands in great need of more thorough treatment in our educational institutions.

The petrographer, again, should seek to have his analyses made as complete as possible, and not, as is so often the case, be content with determinations of silica, alumina, the oxides of iron, lime, magnesia, the alkalies, and water. The latter course, it is true, is entirely justifiable at times, and may serve the immediate purposes of the analyses, but their incompleteness may, on the other hand, not only conceal points fruitful of suggestion to the attentive mind, but, what is of still greater importance, they may be actually misleading. Enough instances of totally inaccurate conclusions to be drawn from them have fallen under my own observation to fully justify this plea in favor of greater completeness in rock and mineral analysis made for purely scientific purposes. ${ }^{a}$

a For a further presentation of this subject from the view point of an experienced petrographer, see Washington, H. S., Manual of the Chemical Analysis of Rocks, 1904, pp. 8-17. 
The importance of the points indicated in the foregoing paragraph. is shown by the difference between the analyses given below. The specimens were taken and analyzed at widely separated times and by different persons, it is true, but they were unquestionably from the same rock mass, in which, however much the relative proportions of the different mineral constituents might vary within certain limits, there can be no reason to doubt the general distribution of all the elements shown by the second analysis.

Analyses of specimens taken from the same rock mass at different dates.

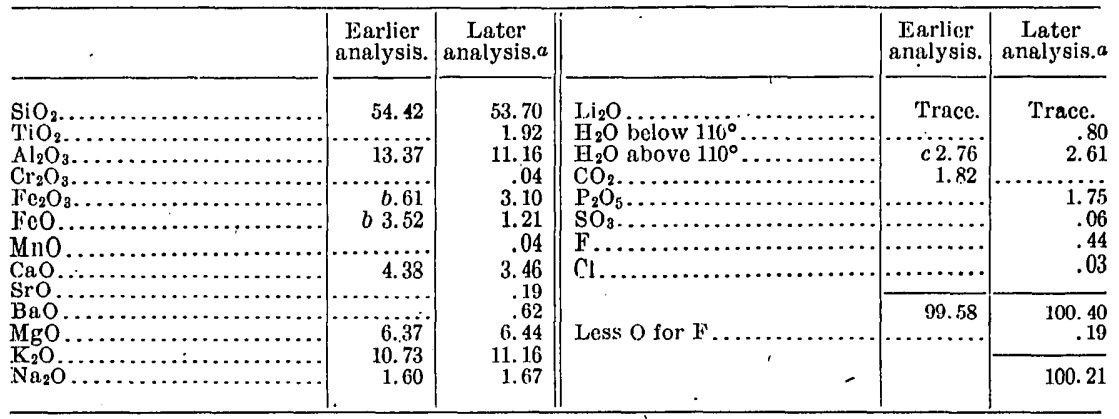

a A still more recent analysis of another of the series of rocks of which this is an example has shown that this "later analysis" is itself probabiy incomplete and incorrect in part-incomplete because of the probable presence of 0.2 per cent or more of $\mathrm{ZrO}_{2}$, incorrect because of the error in $\mathrm{Al}_{2} \mathrm{O}_{3}$ resulting from having counted the $\mathrm{ZrO}_{2} \mathrm{as} \mathrm{Al} \mathrm{O}_{2} \mathrm{O}_{3}$, and from the fact that titanium is not fully precipitable in prosence of zirconium by Gooch's method (the one employed). This latter error involves both the TiOs and the $\mathrm{Al}_{2} \mathrm{O}_{3}$. (See B, p. 136.)

$b$ From the fact that repeated determinations of the iron oxides in this and related rocks from the same region show always a great preponderance of ferric oxide, it is not improbahle that the figures given for the two oxides in the frrst analysis were accidentally transposed.

c In the published analysis it does not appear whether this is total water or, as seems probable, only that remaining above $100^{\circ}$.

Another instance of similar kind is given below. Here, again, certain differences are explainable by natural variations in the proportions of the constituent minerals, but it can hardly be doubted that $\mathrm{TiO}_{2}, \mathrm{BaO}, \mathrm{SrO}, \mathrm{P}_{2} \mathrm{O}_{5}$, and $\mathrm{SO}_{3}$ were present in both specimens in approximately the same amounts. In the earlier analysis determinations of some supposedly unimportant constituents were purposely omitted, or made only qualitatively; with results that can not be otherwise than fatal to a full comprehension of the mineralogical nature of the rock.

Analyses of specimens taken from the same rock mass at different dates.

\begin{tabular}{|c|c|c|c|c|c|}
\hline 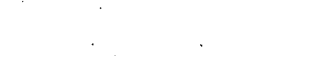 & $\begin{array}{c}\text { Earlier } \\
\text { analysis. }\end{array}$ & $\begin{array}{c}\text { Later } \\
\text { analysis. }\end{array}$ & . & $\begin{array}{c}\text { Earlier } \\
\text { analysis. }\end{array}$ & $\begin{array}{c}\text { Later } \\
\text { analysis. }\end{array}$ \\
\hline $\mathrm{SiO}_{2}$ & 44.31 & 44.65 & $\mathrm{Na}_{2} \mathrm{O}$. & 4.45 & 5.67 \\
\hline (1, & Not est. & .95 & $\mathrm{Li}_{2} \mathrm{O} \ldots \ldots \ldots$ & so & Trace. \\
\hline $\mathrm{Al}_{\mathrm{Fe}_{2} \mathrm{O}_{3}} \ldots \ldots \ldots \ldots \ldots \ldots \ldots \ldots \ldots \ldots \ldots \ldots$ & 17.20 & - 13.87 & $\mathrm{H}_{2} \mathrm{O}$ below $110^{\circ}$.. & .77 & .95 \\
\hline $\begin{array}{l}\mathrm{Fe}_{2} \mathrm{O}_{3} \\
\mathrm{FeO}\end{array}$ & $\begin{array}{l}4.64 \\
3.73\end{array}$ & $\begin{array}{l}6.06 \\
2.94\end{array}$ & $\mathrm{H}_{2} \mathrm{O}$ by ignition.... & 3.30 & \\
\hline $\mathrm{MnO} .$. & .10 & .17 & $\mathrm{CO}_{3} \ldots \ldots \ldots \ldots \ldots$ & ....... & \\
\hline 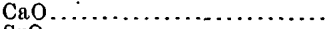 & 10.40 & 9.57 & $\mathrm{P}_{2} \mathrm{O}_{3} \ldots$ & .... & 1.50 \\
\hline ………, & ....... & $a .37$ & $\mathrm{Cl} . . . . .$. & & Trace. \\
\hline MgO. & 6.57 & 5.15 & $\mathrm{SO}_{3} \ldots .$. & & \\
\hline $\mathrm{K}_{2} \mathrm{O}$. & 3.64 & 4. 49 & - & 99.11 & 99.92 \\
\hline
\end{tabular}

$a$ Not entirely free from $\mathrm{CaO}$. 
Prof. F. W. Clarke has shown that the combined percentages of titanic and phosphoric oxides in rocks of the earth's crust, averaged from hundreds of analyses, amount to 0.8 per cent. When the determination of these is neglected the error falls on the alumina. If the alumina is then used as a basis for calculating the feldspars, it is easy to see that a viery large average error in the latter may result, amounting to several per cent of the rock.

In order to emphasize more strongly the importance of completeness in analysis, a few facts brought out by the hundreds of rock analyses made in this laboratory may be cited. It has been demonstrated most conclusively that barium and strontium are almost never-failing constituents of the igneous rocks of the United States and of many of their derivatives. The amounts are usually below 0.1 per cent for each of the oxides of those metals, but higher amounts are by no means uncommon. Furthermore, the weight of barium is almost without exception in excess of that of strontium; but a still more important point is that the igneous rocks of the Rocky Mountain region, so far as examined, show far higher average percentages of both metals than the rocks from the eastern and the more western portions of the United States. The following examples serve to illustrate certain types of Rocky Mountain igneous rocks: Of seven rocks forming a Colorado series, six held from 0.13 to 0.18 per cent of $\mathrm{BaO}$, while in the seventh the percentage was 0.43 . The SrO ranged from 0.07 to 0.13 per cent for six and was 0.28 for that one highest in $\mathrm{BaO}$. Of thirteen geologically related rocks from Montana, embracing basic as well as acidic and intermediate types, the range of $\mathrm{BaO}$ was from 0.19 to 0.37 per cent, with an average of 0.30 per cent. Three others of the same series contained 0.10 per cent or less, while the seventeenth carried 0.76 per cent $\mathrm{BaO}$. The $\mathrm{SrO}$ ranged from 0.37 per cent in the last instance to an average of 0.06 for the other sixteen. Certain peculiar rocks from Wyoming carry from 0.62 to 1.25 per cent $\mathrm{BaO}$ and from 0.02 to 0.33 per cent SrO. Surely this concentration of certain chemical elements in certain geographic zones has a significance which future geologists will be able to interpret if those of to-day are not.

Again, vanadium is an element which few chemists have ever thought of looking for in igneous rocks, though it has long been: known to occur in magnetites and other iron ores. A. A. Hayes, in 1875 , reported its occurrence in a great variety of rocks and ores. To quote from Thorpe's Dictionary of Chemistry: "It is said to be diffused with titanium through all primitive granite rocks (Dieulafait), and has been found by. Deville in bauxite, rutile and many other minerals and by Bechi and others in the ashes of plants and in argillaceous limestones, schists, and sands." It is further reported to 
comprise, as the pentoxide, up to 0.1 per cent of many French and Australian $^{a}$ clays, 0.02 to 0.03 per cent of some basalts, 0.24 per cent of a coal of unknown origin, and 0.45 per cent of a coal from Peru. Still later examinations in this laboratory of about 100 rocks, chiefly igneous, covering nearly the whole continental territory of the United States, show not only its general qualitative and quantitative distribution, but that it predominates in the less siliceous igneous rocks and is absent, or nearly so, in those high in silica. In some of the more basic rocks it occurs in sufficient amount to affect seriously the figures for the oxides of iron unless separately estimated and allowed for (see $\beta$, p. 166), a matter of considerable importance, since the petrographer lays great stress on accuracy in their determinations.

The same investigation has also thrown some light on the distribution of molybdenum, which seems to be confined to the more siliceous rocks and to occur in quantities far below those commonly found for vanadium.

Finally, had it not been my practice of late years to look for sulphur in rocks, even when no sulphides were visible to the eye, its almost invariable presence in the form of sulphide and consequent connection with the long mystifying lack of agreement between results for ferrous iron obtained by the Mitscherlich and the hydrofluoric-acid methods might not have been suspected (see B, p. 157).

While strongly upholding the necessity for more thorough work, necessarily somewhat at the expense of quantity, it is far from my intention to demand that an amount of time altogether disproportionate to the immediate objects to be sought should be expended on every analysis. But it is maintained that, in general, the constituents which are likely to be present in sufficient amount to admit of determination in the weight of a sample usually taken for analysis-say 1 gram for $\mathrm{SiO}_{2}$, $\mathrm{Al}_{2} \mathrm{O}_{3}$, etc., to 2 grams for certain other constituents-should be sought for, qualitatively at least, in the ordinary course of quantitative work, and their presence or absence noted among the results. If present in little more than traces, that knowledge alone may suffice, for it is often more important to know whether or not an element is present than to be able to say that it is there in amount of exactly 0.02 or 0.06 per cent. In the tabulation of analyses a special note should be made in case of intentional or accidental neglect to look for substances which it is known are likely to be present. Failure to do this may subject the analyst to unfavorable criticism when, at some future time, his work is reviewed and the omissions are discovered by new analyses.

Finally, whenever possible, a thorough microscopical examination of the rock in thin section should precede the chemical analysis. This

a J.C. H. Mingaye has lately confirmed its wide distribution in Australian rocks, coals, etc. (Records Geol. Survey New South Wales, vol. 7, pt. 3, 1903, p. 213.) 
may be of the greatest aid to the chemist in indicating the presence of unusual constituents or of more than customary amounts of certain constituents, whereby, possibly, necessary modifications in the analytical procedure may be employed without waste of time or labor. ${ }^{a}$

If the point be raised that many of the published analyses emanating from the Survey laboratories, even my own earlier ones, are not in accord with the advocacy of completeness contained in the foregoing pages, it may be remarked that these ideas have been to a considerable degree evolved during a personal experience of over twentyfive years in this line of work, and that frequently the exigencies were such as to compel restriction in the examination. Where the latter has been the case, subsequent developments have in some cases shown it to be bad policy in every respect. It is better, for both the geologist and the chemist, to turn out a limited amount of thorough work than a great deal of what may ultimately prove to be of more than doubtful utility.

\section{THE CONSTITUENTS OCCURRING IN SILICATE ROCKS.}

It is to the decomposition of the original igneous rocks or their magmas and their derivatives that nearly all ore bodies in the United States owe their origin by one or another process of concentration. A certain class of concentrations probably separated from magmas in the fluid state before solidification. Hence it is the natural and inevitable inference that sufficiently careful examination of these rocks would show them to contain all or nearly all the known elements, not necessarily all in a given rock, but many more than anyone has yet found. ${ }^{b}$ Mechanical and analytical difficulties have thus far stood in the way of experimental proof that this is so, owing to the great scarcity of many of the elements. In exceptional cases it may be desirable to subject a given material to very exhaustive analysis, as, for instance, when searching for the origin of ore deposits. Ordinarily, however, the demands of the petrographer and geologist are satisfied with a knowledge of the quantitative relations of those constituents which can be determined in a limited amount of the sample-say from one-half gram to 2 , or occasionally 5 , grams. In general the discussion relating to silicate analysis in this volume will be confined to such separations as may be required in the analysis of an igneous, metamorphic, or sedi-

\footnotetext{
a See also Washington, H. S., Chemical Analysis of Rocks, 1904, pp.6-7. The foregoing tables and accompanying remarks, including several sentences preceding the tables, have been largely taken from my paper entitled A plea for greater completeness in chemical rock analysis: Jour. Am. Chem. Soc., vol. 16, 1894, pp. 90-93; Chem. News, vol. 69,1894 , p. 163 . See also Distribution and quantitative occurrence of vanadium and molybdenum in rocks of the United States: Am. Jour. Sci., 4th ser., vol. 6, 1898, p. 209; Chem. News, vol. 78, 1898, p. 216; and Bull. U.S. Geol. Survey No. 167, 1900, p. 49.

$b$ F. Sandberger's researches have shown to what extent this is true of a large number of those elements contributing to the filling of metalliferous veins, and L. Dieulafait by his elaborate qualitative researches showed how universal was the distribution of copper, zinc, barium, strontium, etc., in the primordial rocks.
} 
mentary rock of complex mineralogical composition, in which the majority and possibly all of the ingredients in the list given below may occur in weighable or readily discoverable quantities:

$\mathrm{SiO}_{2}, \mathrm{TiO}_{2}, \mathrm{ZrO}_{2}, \mathrm{Al}_{2} \mathrm{O}_{3}, \mathrm{Fe}_{2} \mathrm{O}_{3}, \mathrm{Cr}_{2} \mathrm{O}_{3}, \mathrm{~V}_{2} \mathrm{O}_{3}, \mathrm{FeO}, \mathrm{MnO}, \mathrm{NiO}, \mathrm{CoO}$, $\mathrm{MgO}, \mathrm{CaO}, \mathrm{SrO}, \mathrm{BaO}, \mathrm{ZnO}, \mathrm{CuO}, \mathrm{K}_{2} \mathrm{O}, \mathrm{Na}_{2} \mathrm{O}, \mathrm{Li}_{2} \mathrm{O}, \mathrm{H}_{2} \mathrm{O}, \mathrm{P}_{2} \mathrm{O}_{5}, \mathrm{~S},{ }^{a}$ $\mathrm{SO}_{3}, \mathrm{C}, \mathrm{CO}_{2}, \mathrm{Fl}, \mathrm{Cl}, \mathrm{N}$.

To the above list might be added certain others, as the group of so-called rare earths, besides tin, platinum, tantalum, columbium, boron, glucinum, ${ }^{c}$ helium. Some of these occur at times in determinable amounts, though in my experience tantalum, columbium, and glucinum have not been met with. They may very well have been overlooked by reason of the absence of distinctive tests for identification. Thorium, cerium, and other rare earths are probably more common as constituents of silicate rocks than has been generally supposed. Their presence and amount can be so readily and certainly detected by the methods given in their proper place that the reason for neglecting to look for them is no longer so strong as it used to be, especially when there is microscopic or other evidence of the presence of minerals likely to contain them.

\section{THE DISTRIBUTION AND OCCURRENCE OF CERTAIN MINOR CONSTITUENTS.}

For the suggestion of this section and for much of its contents I am in no small degree indebted to Doctor Washington's book (Manual of the Chemical Analysis of Rocks, pp. 18-21), already more than once referred to.

Doctor Washington says:

"The increased number of analyses of igneous rocks, especially of unusual types, and the more frequent determination of the minor constituents, with the vast mass of data obtained by the use of the microscope, have shown that certain of the rare elements are prone to occur in rocks of certain chemical characters. While our knowledge along this line is far from complete, a few words may be devoted to this subject, as it will often be of use to the analyst to know which elements should be especially looked for and which may be safely neglected."

Titanium.-Not long ago held to be rare, this element is now recognized to be one of the most universally distributed and about tenth in actual abundance in the explored crust of the earth. So far as my experience goes, it is entirely absent from no igneous, metamorphic, or sedimentary rock of a more or less siliceous character. The recognition of this fact has been rendered possible and easy by its characteristic behavior, when in solution, toward hydrogen peroxide. Though

a Usually as pyrite, occasionally as lazurite, not infrequently as pyrrhotite.

$b$ As graphite or coaly matter.

$c$ For probably the best method of separating even very small amounts of glucinum from iron and aluminum see Parsons, C. L., Jour. Am. Chem. Soc., vol. 28, 1906, p. 1589; Chem. News, vol. 95, 1907, p. 188. 
seemingly present even in the most siliceous rocks, it is most abundant in the so-called basic ones. Its chief mineral occurrences are as rutile, octahedrite or anatase, ilmenite, titanite, and perofskite, but it is also a component in smallêr amounts of many pyroxenes, hornblendes, biotites, and garnets. Owing to the refractory nature of some of its compounds it tends to concentrate in the residual products of decomposition of many rocks; hence its high percentage in clays as a class. It is very unusual to find titanium present in amounts that can properly be designated only as traces. In the great majority of cases its amount will not exceed 1 per cent, but it may rise to over 5 per cent.

Zirconium.-The chemically related element zirconium is likewise now known to be more widely distributed than was formerly supposed, but it is much less common than titanium, rarely making up 0.2 per cent of a rock and being usually under 0.05 per cent. "It is most apt to occur in granites, rhyolites, syenites, and in nephelite syenites, phonolites, tinguaites, and tephrites, and is most abundant in those which are high in soda, such as the last four. It is rarely met with in the more basic rocks, especially those rich in lime, magnesia, and iron. Zirconium is usually found as the silicate zircon, especially in granites and syenites, but is also an ingredient of the rare minerals eudialyte, låvenite, and rosenbuschite."

Chromium.- "Chromium is almost wholly confined to the basic rocks, especially those which are high in magnesia and low in silica, and consequently contain abundant olivine, such as peridotite and dunite. It occurs as chromite and picotite (chrome-spinel), and in some augites, biotites, and olivines. It may occur up to one-half of 1 per cent of $\mathrm{Cr}_{2} \mathrm{O}_{3}$."

Vanadium.-The distribution of this element has been in part covered by the remarks on pages 20-21. To leave out of account the wellcharacterized mineral vanadates, its most stable and normal form in the silicates seems to be that of trivalency, corresponding to the oxide $\mathrm{V}_{2} \mathrm{O}_{3}$, in which form it replaces alumina and possibly ferric oxide to a very limited extent - a few hundredths of one per cent-in pyroxenes, hornblendes, and biotites. It hence predominates in the less siliceous igneous rocks. As the mineral roscoelite, essentially a vanadioalumino-potassic silicate, or some other similar silicate, it is rather widely distributed as a component of certain sandstones in western Colorado and eastern Utah, sometimes in large amount. ${ }^{a}$ It may well exist in a similar condition in clays and the ashes of coals. The conclusion being based on the above observations, it should not occur as a constituent of nonaluminous and nonferric rock-forming minerals.

$a$ Hillebrand, W. F., and Ransome, F. L., Am. Jour. Sci., 4th ser., vol. 10, 1900, p. 120; Bull. U. S. Geol. Survey No. 262, 1905, p. 9. 
As to olivine, at least, this conclusion has been found to be justified. Vanadium is also a constituent of ilmenite in titaniferous iron ores. The existence of the copper sulphovanadate, sulvanite, and the occurrence of vanadium in apparent combination with sulphur in certain peculiar carbonaceous ores of eastern Utah and Peru ${ }^{a}$ indicate a possible wider range of combination in exceptional cases.

Manganese.-This element is found in ferromagnesian minerals in nearly all rocks, though as the result of their alteration it may sometimes appear, particularly on surfaces of limestones and sandstones, in a more or less peroxidized condition. Its amount will rarely exceed 0.3 per cent. I fully agree with Doctor Washington in regarding the high figures commonly reported as due to analytical error. There is no element which the average chemist is more prone to report too high, by gravimetric methods.

Nickel and cobalt.-These elements are found in the olivine of peridotite rocks and also in pyrite and pyrrhotite, hornblende, and biotite. Even in peridotites the percentage of nickel will seldom exceed 0.1 per cent, while that of cobalt rarely if ever exceeds a trace. As with manganese, the percentage of nickel has often been reported too high.

Copper.-Owing to the extreme precautions necessary to exclude its introduction from utensils and reagents during analysis, there is less positive information regarding the distribution of copper than of some other elements which do not exceed it in amount. Nevertheless, there is reason to believe that it is almost if not quite as universally distributed as most of the other minor constituents. Its particular home appears to be in diabase, gabbro, amphibolite, and other basic rocks with pyroxene and amphibole. J. B. Harrison, in a recent report (1906) to the Science and Agriculture Department of British Guiana, shows its occurrence in hundredths of 1 per cent in many igneous rocks of that colony. So far as my experience goes, it can be found almost invariably, if looked for, in the rock analyses carried out in the Survey laboratory. But for the reason above given it is seldom reported unless extra precautions have been taken to prevent its extraneous entry into the analysis.

Barium and strontium.- Feldspathic rocks are those most likely to carry these elements, the former almost always in excess of the latter. According to Washington, there is considerable evidence, in part unpublished, that barium is apt to be most abundant in 'rocks which are high in potassium. It occurs "in orthoclase (as the hyalophane molecule) and possibly also in labradorite and anorthite (as celsian) as well as in a few biotites and muscovites." It has been found in a few peculiar rocks in excess of 1 per cent, in terms of the oxide, but in the greater part of the silicate rocks of the United States its percent-

$a$ As to the Peruvian occurrence see Hewett, Foster, Eng. Min. Jour., vol. 82, 1906, p. 385; Bravo, José J., Bol. Soc. Ing. (Lima), vol. 8, 1906, p. 171; Hillebrand, W. F., Am. J,our. Sci., 4th ser., vol. 24, 1907, p. 141; and Jour. Am. Chem. Soc., vol. 29, 1907, p. 1019. 
age is well below 0.2. Strontium has been found as high as 0.3 to 0.4 per cent, but generally there is little more than a trace of it.

Lithium.-Although one of the most universally distributed of the elements, it is almost never met with in rocks in more than spectroscopic traces. Aside from the lithium minerals lepidolite and spodumene, it is found in the alkali feldspars, in muscovite, beryl, and other minerals. According to Washington, there is reason for the belief that it is especially prone to occur in highly sodic rocks.

Phosphorus.-This element is found in greatest abundance in the more basic igneous and metamorphic rocks, and is practically never entirely absent. It especially affects those rocks "which are high in lime and iron rather than in magnesia." Its chief mineral occurrence is in apatite, though it may be found in xenotime and monazite. While the percentage is usually well under 1 , it may considerably exceed this figure.

Sulphur.-Aqueous extraction of a powdered rock will in manycases remove traces of sulphur and chlorine. These are to be regarded in almost every case rather as derived from infiltrating waters than as products of decomposition of the constituents of the rock itself. Essential rock-forming minerals containing sulphur in the oxidized condition are limited to hauynite and noselite, minerals which are found chiefly in the more basic rocks, and especially those high in soda. In the sulphide condition the element is of very wide distribution, both as pyrite and pyrrhotite, less often as chalcopyrite and possibly other sulphides, also in the mineral lazurite, and here too in greater abundance in the basic rocks. It is a very common error of most chemists to report the sulphur in silicate and carbonate rocks, clays, etc., as $\mathrm{SO}_{3}$ instead of $\mathrm{S}$. It may now and then occur in both states, but much more often only in the sulphide condition.

Chlorine. - "Chlorine is present most abundantly in rocks which are high in soda, and especially when so low in silica that nephelite is present, though it is also found sometimes in nephelite-free rocks, and in a few cases in quartz-bearing ones. It is an essential component of sodalite [also noselite--W. F. H.], and is also present in scapolite and in a few apatites." Its amount when present rarely exceeds 0.2 or 0.3 per cent. When seeking for the chlorine of these minerals it is always well to extract the powder first with cold water (see preceding paragraph). It is to be remembered that fluid inclusions in minerals sometimes contain sodium chloride, which would be largely extracted from the powder by water.

Fluorine.- "Fluorine seems to have no special preference as to magma, though, on the whole, it is found more frequently in acid than in basic rocks. It is also apparently most apt to be met with as fluorite in rocks containing nephelite, as foyaites and tinguaites. It is an essential constituent of fluorite and most apatite, and as an integral 
part of the last mineral is almost universally present. It also occurs in biotites and other micas, in some hornblende and augite, as well as in tourmaline, topaz, chondrodite, etc." When determined it is usually reported in amounts under 0.1 per cent, but freedom of the weighed calcium fluoride from contandination being assumed, the reported amount is invariably too low because of the inaccuracy of the method for its separation (see c, p. 187).

Other minor constituents.- "Glucinum, as a component of beryl, is most frequent in granites, pegmatites, and quartzose gneisses. Tin is confined to the acid rocks, granite, quartz porphyry, and rhyolite, and its presence is due [often-W. F. H.] to pneumatolytic processes. It occurs as cassiterite, and in traces in ilmenite, micas, and feldspars. The rare-earth metals occur in allanite, xenotime, monazite, and other minerals of even greater rarity, and seem to be especially frequent in acid rocks and possibly those with much soda. Molybdenum tungsten, and uranium are almost exclusively confined to the very siliceous rocks. Zinc has been met with in granite, as well as in basic rocks, but no generalization in regard to it is possible as yet [nor in regard to lead, which can often be found by using considerable amounts of rock material, as in some of the rocks of Leadville, Colo., and in those of British Guiana.-W. F. H.] Platinum is, found almost exclusively in peridotites, but is occasionally met with in connection with gabbros. Boron, as a constituent of tourmaline, is most apt to occur in highly siliceous rocks." Boron is also common in many contact-metamorphic schists. Gold and silver have been found repeatedly. According to Harrison, ${ }^{a}$ some of the British Guiana rocks contain gold enough to account for the commercially valuable teposits in residual soils resulting from the rock decomposition. Tantalum and columbium occur in some granites and pegmatites, but have never been reported in percentages in rock analysis, so far as known to me.

\section{千. SUMMATION OF ANALYTICAL RESULTS AND LIMITS OF ALLOWABLE ERROR.}

As is well known, a complete silicate-rock analysis which foots up less than 100 per cent is generally less satisfactory than one which shows a summation somewhat in excess of 100 . This is due to several causes. Nearly all reagents, however carefully purified, still contain, or extract from the vessels used, traces of impurities, which are eventually weighed in part with the constituents of the rock. The dust entering an analysis from first to last is considerable, washings of precipitates may be incomplete, and if large filters are used for small precipitates the former may easily be insufficiently washed. 
Given the purest obtainable reagents, an ample supply of platinum, facilities for working, and a reasonably clean laboratory, there is usually little excuse for failure on the part of a competent chemist to reach a summation within the limits 99.75 and 100.50. 。Failure to attain 100 per cent in several of a series of analyses of similar nature should be the strongest evidence that something has been overlooked. Excess above 100.5 per cent should be good ground for repeating portions of the analysis in order to ascertain where the error lies, for it is not proper to assume that the excess is distributed over all determined constituents. It is quite as likely, in fact more than likely, to affect a single determination and one which may be of importance in a critical study of the rock from the petrographic side.

Doctor Washington would extend the allowable limits above given to 99.50 and 100.75 . If the analysis has to be made in other vessels than those of platinum a higher limit than 100.50 is certainly called for, but it is extremely doubtful if a lower one than 99.75 should be accepted, for, as has been said, there is a tendency toward too high results in any case, and a summation of less than 99.75 with vessels other than platinum would be indicative of rather gross error on the part of the analyst.

As to the allowable variation in duplicate determinations of the same constituent no rigid rules can be laid down. Doctor Washington has proposed the following, ${ }^{a}$ with which the independent experimental values of Doctor Dittrich ${ }^{b}$ are in substantial agreement: For. $\mathrm{SiO}_{2}$ and others. which amount to 30 per cent and over, from 0.2 to 0.3 per cent; for $\mathrm{Al}_{2} \mathrm{O}_{3}$ and others which amount to from 10 to 30 per cent, 0.1 to 0.2 per cent; for constituents which amount to from 1 to 10 per cent, 0.05 to 0.1 per cent. These percentages are in terms of the whole rock and not of the particular constituent.

While it is desirable sometimes, and for the beginner imperative, to make duplicate determinations, agreement is not to be taken as proof that the results are correct unless they have been arrived at by different methods.

Too great stress can not be laid upon the importance, especially for the analyst of limited experience, of consistently testing most of his final precipitates and filtrates as carefully as possible in order to be sure, on the one hand, that the substances reported by a given name are wholly such and, on the other, that all is reported. In no other way can an analyst so soon acquire the needed confidence in himself and his methods.

a Manual of the Chemical Analysis of Rocks, 1904, p. 24.

$b$ Neues Jahrb. f. Min. u. Geol., vol. 2, 1903, p. 69. 


\section{STATEMENT OF ANALYSES.}

Until recently it has been the practice in this laboratory to tabulate the constituents of a rock somewhat in the order of their determination, beginning with $\mathrm{SiO}_{2}$ as the chief constituent and grouping together all chemically related oxides, as shown, for instance, on page 19.

From a strictly scientific point of view a chemical classification - founded on a separation into basic and acidic atoms or radicals would be more satisfactory, but until we learn to find out what silicic radicals are present and in what relative amounts, also how much free silica there may be, it is useless to think of employing the arrangement so valuable in stating water analyses.

Of late, petrographers have begun to demand, with considerable reason, an arrangement "which shall bring the essential chemical features-both the percentage figures and the molecular ratios-prominently and compactly before the eye, so that the general chemical character and the relations of the various constituents may be seen at a glance." $a$

In accordance with this demand it is now our practice to follow pretty closely the arrangement proposed by Pirsson and later strongly advocated by Washington, ${ }^{b}$ namely:

$\mathrm{SiO}_{2}, \mathrm{Al}_{2} \mathrm{O}_{3}, \mathrm{Fe}_{2} \mathrm{O}_{3}, \mathrm{FeO}, \mathrm{MgO}, \mathrm{CaO}, \mathrm{Na}_{2} \mathrm{O}, \mathrm{K}_{2} \mathrm{O}, \mathrm{H}_{2} \mathrm{O}$ (above $105-$ $110^{\circ}$ ), $\mathrm{H}_{2} \mathrm{O}$ (below $105-110^{\circ}$ ), $\mathrm{CO}_{2}, \mathrm{TiO}_{2}, \mathrm{ZrO}_{2}, \mathrm{P}_{2} \mathrm{O}_{5}, \mathrm{SO}_{3}, \mathrm{Cl}, \mathrm{Fl}$, $\mathrm{S}\left(\mathrm{FeS}_{2}\right), \mathrm{Cr}_{2} \mathrm{O}_{3}, \mathrm{~V}_{2} \mathrm{O}_{3}, \mathrm{NiO}, \mathrm{CoO}, \mathrm{CuO}, \mathrm{MnO}, \mathrm{SrO}, \mathrm{BaO}, \mathrm{Li}_{2} \mathrm{O}, \mathrm{C}, \mathrm{NH}_{3}$.

By this arrangement the nine constituents which in the great majority of cases determine the character of the rock are placed at the head of the list, thus greatly facilitating the comparison of different analyses similarly arranged, especially when, as Washington recommends, the molecular ratios are calculated for these leading constituents and placed immediately after the corresponding oxides. The order of the remaining members is determined somewhat by the following considerations: $\mathrm{CO}_{2}$ is placed next after $\mathrm{H}_{2} \mathrm{O}$, since these two are generally a measure of the alteration the rock may have undergone. $\mathrm{TiO}_{2}$ and $\mathrm{ZrO}_{2}$ naturally follow $\mathrm{CO}_{2}$ on chemical grounds, and $\mathrm{SO}_{3}$ and $\mathrm{Cl}$, being common constituents of the sodalite group, are conveniently placed together.

It may be said with regard to the use of the word "trace" that the amount of a constituent thus indicated is supposed to be below the limit of quantitative determination in the amount of the sample taken for analysis. It should in general, for analyses laying claim to completeness and accuracy, be supposed to indicate less than 0.02 or even 0.01 per cent.

a Washington, H. S., The statement of rock analyses: Am. Jour. Sci., 4th ser., vol. 10, 1900, p. 61. $b$ Loc. cit. 


\section{TIME NEEDED FOR MAKING AN ANALYSIS.}

The question has often been put, "How long does it take to complete an analysis of this kind?" This will depend, of course, on the mineral complexity of the sample and on the personal factor of the individual worker. If there is a competent assist-

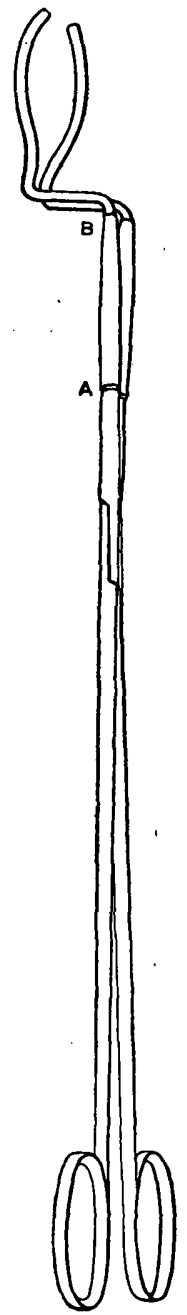

FIG. 1.-Platinum-tipped crucible tongs. The parts $A, B$, also of heavy platinum, are hollow, to serve as sockets for the cheaper metal of the handles. ant to do the grinding, and specific-gravity determinations are not required, it is quite possible after long experience for a quick worker to learn to so economize every moment of time in a working day of seven hours, with an abundance of platinum utensils and continuous use of air and water or steam baths through the night, as to finish every three days, after the completion of the first analysis, barring accidents and delays, one of a series of rocks of generally similar character, each containing from eighteen to twenty quantitatively determinable constituents excluding, for instance, fluorine, carbon as such, nitrogen, metals of the hydrogen sulphide group, and cobalt. But such an output of work implies an unusual freedom from those occasional setbacks to which every chemist is exposed.

\section{USEFUL APPLIANCES AND APPARATUS.}

In connection with the foregoing remarks it is in place to mention a few aids to the chemist which are in constant use in this laboratory and have come to be well-nigh indispensable. None is novel in principle and all are in use elsewhere, but some are not so commonly known as they deserve to be, hence this allusion to them. Certain cheap and simple forms of colorimeters have been found very useful, and since they are adapted to the determination of a variety of substances their description finds most appropriate place here.

\section{A. CRUCIBLE TONGS.}

Fig. 1 represents a form of platinum-tipped crucible tongs devised by Dr. A. A. Blair many years ago. With them a crucible can be securely grasped and brought into any desired position while still hot. To the contents, if in fusion over the blast flame, can be imparted the 
rotatory motion so often desirable. Above all, the cover need not be in the slightest degree displaced, as when using the common form of platinum-tipped tongs.

\section{B. RADIATORS FOR VOLATILIZING LIQUIDS AND SOL.IDS.}

Fig. 2 represents a very useful adjunct to the worktable and especially to the draft cupboard, whereby the liquid contents of crucibles can be speedily evaporated at almost any desired temperature and the

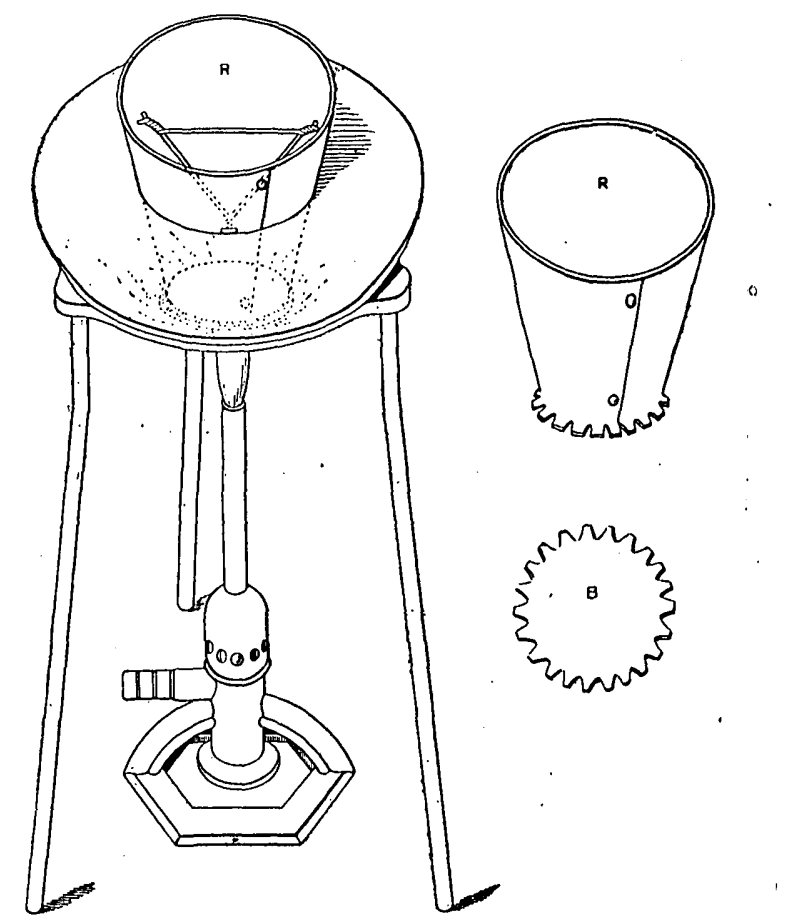

FIG. 2.-Radiator for rapid and safe evaporation. $R$ is of sheet iron or aluritinum, also nickel (Jannasch). A convenient height is $7 \mathrm{~cm}$, width at top $7 \mathrm{~cm}$. and at bottom $5 \mathrm{~cm}$. The base, $B$, may be of iron, nickel, or platinum, but not aluminum, which will not stand the temperature of the direct flame. Platinum is most satisfactory by reason of its long life and radiating power. The manner of attaching $B$ to $R$ is by turning the cogs of $B$ up and over those of $R$. Evaporation may be greatly hastened when desired by placing upon $R$ a cast-iron ring with its opening somewhat larger than the mouth of the underlying crucible.

dehydration of many solids effected much more safely than on an iron plate or sand bath. I do not recall who originated this form of air bath, but it has been in use here for over twenty years and is identical in principle with the "Nickel-becher" of Jannasch. Nickel undoubtedly has a certain advantage in not rusting as does iron, but the form depicted in fig. 2 can easily be made anywhere of sheet iron riveted at the joint; the bottom being securely held by a notched flange at the extremity of the truncated cone. A crucible placed on the platinum triangle. becomes uniformly heated by hot air, and large quantities of 
liquid, even sulphuric acid, can be thus volatilized in a short time without ebullition or spattering. The life and effectiveness of this appliance is greater if the bottom is of sheet platinum, but if an iron

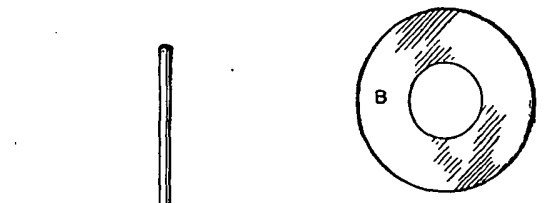
bottom wears out it is usually possible to replace it one or more times before the sides fail. A coating of aluminum paint on the sides adds to their life.

By an extension of the principle illustrated in fig. 2 very considerable amounts of ammonium salts and other easily volatile solids may be driven off from platinum dishes with little danger of loss by spattering and none by.overheating. The dish containing the dry or nearly dry matter is placed in another of such size that the bottom of the inner dish is at some distance from that of the outer. The outer dish may be of iron, direct contact between it and the inner dish being prevented by wisps of asbestos overlapping the edge of the former, but the excellent conductivity and radiation of platinum recommends it above anything else. Aluminum will not stand the application of a full

Fig. 3.-Device for excluding flame gases from the interior of crucibles during ignition. A, Asbestos board; B, stiff platinum foil of sizo suitable to the crucible used and having an opening to admit the crucible to about twothirds of its depth. This disk may be used alone upon the ring of an ordinary stand and in horizontal position, or, preferably as shown in the figure, in combination with the asbestos board, in which is an opening considerably larger than the crucible. The combination should then be given the inclined position shown, so as to allow the products of combustion to flow to one side without the possibility of their enveloping the mouth of the crucible. In the lack of platinum a perforated asbestos board alone will yield fair service with a blast, !but not as a rule with an ordinary burner.

flame. If the contents of the dish are moist at first, a low flame will soon dry them, when the heat may be increased at will and the operation allowed to proceed without supervision. A sand bath may sometimes be substituted for the radiator.

\section{PERFORATED DISK FOR CRU-} CIBLES.

Fig. 3 shows an arrangement for the ignition of crucibles when it is desired to exclude flame gases from their interior, as in the ignition of ferric oxide and the determination of sulphur by fusion with an 
alkali carbonate. The original idea of J. Löwe ${ }^{a}$ was to use perforated clay disks in determining the ash of coals. Later, Lunge and others advocated asbestos board, but this has the disadvantage that it lasts but a short time and the fibers adhere to the crucible to some extent. By neither of these is it easy to secure a very high temperature in the crucible. Therefore a disk of platinum is preferable because of its durability, cleanliness, and high conducting power, though its cost may preclude having a set to fit different sizes of crucibles. The platinum disk and asbestos board may be conveniently used in combination, as in the figure. The asbestos then has a large hole, over which a smaller perforated disk of platinum is laid.

\section{WEIGHING SCOOP OR TROUGH.}

Fig. 4 represents a most convenient receptacle for the sample that is being weighed on the balance. It is made of platinum and is counterpoised by a leaden weight. It has the advantage over a watch glass in that its shape permits of ready introduction of its contents into narrow-mouthed receptacles when desired.

\section{E. COLORIMETERS.}

\section{a. FIRST SURVEY FORM.}

The Survey form of colorimeter shown in fig. 5 consists of two glass reservoirs and a darkened box. The glasses, $G$, may be of square or rectangular section, 8 to $12 \mathrm{~cm}$. high and 3 to $3 \frac{1}{2} \mathrm{~cm}$. inside measurement between those sides through which the liquid is to be observed. ${ }^{b}$ These sides should, of course, be exactly parallel, the others need not be, but should be blackened externally. In order to exclude further the effect of side light, it is very convenient to have a simple light box, B, that can be easily held in the hand, stained black inside and out and with one end closed by a piece of ground glass, W, the other being open. For a space equal to the width of the glasses the cover is removed at the top next the glass end to permit the insertion of the glasses side by side in such a way that no light shall penetrate around their sides or between them. Immediately back of the glasses is a partition, $\mathrm{P}$, with openings of appropriate size

a Zeitschr. anal. Chemie, vol. 20, 1881, p. 224.

$b$ The allowable error in distance between the corresponding pairs of sides of the two glasses should not in any case exceed 1 per cent. Unfortunately there has been a disinclination on the part of dealers in this country to furnish glasses fulfiling this requirement, and held together by a durable cement which shall be proof against dilute sulphuric acid and alkali, though some have been obtained through Messrs. Eimer \& Amend, of New York City. Canada balsam answers well for a time, but sooner or later it cracks, leaks then appear, and the sides soon drop off. It is, however, but a simple matter to cement them on again. But the use of Canada balsam is precluded with alkaline solutions, and hence for the chromium determination, as the solution at once becomes turbid. 
cut in it. A stiffly sliding black cardboard shutter, S, is movable up and down immediately back of the partition, so that all light can be cut off except that which comes through the liquid. The ground-glass window, W, sliding up and down in slots, affords a much more uniform illumination than can be had without it.

Precautions of this kind are necessary if accurate results are to be counted on. Except for mere traces, this combination of glasses and darkened box insures greater accuracy and rapidity of work than Nessler tubes, and is preferable likewise, so far as my experience goes, to expensive instruments like the colorimeter of Soleil-Duboscq, etc.
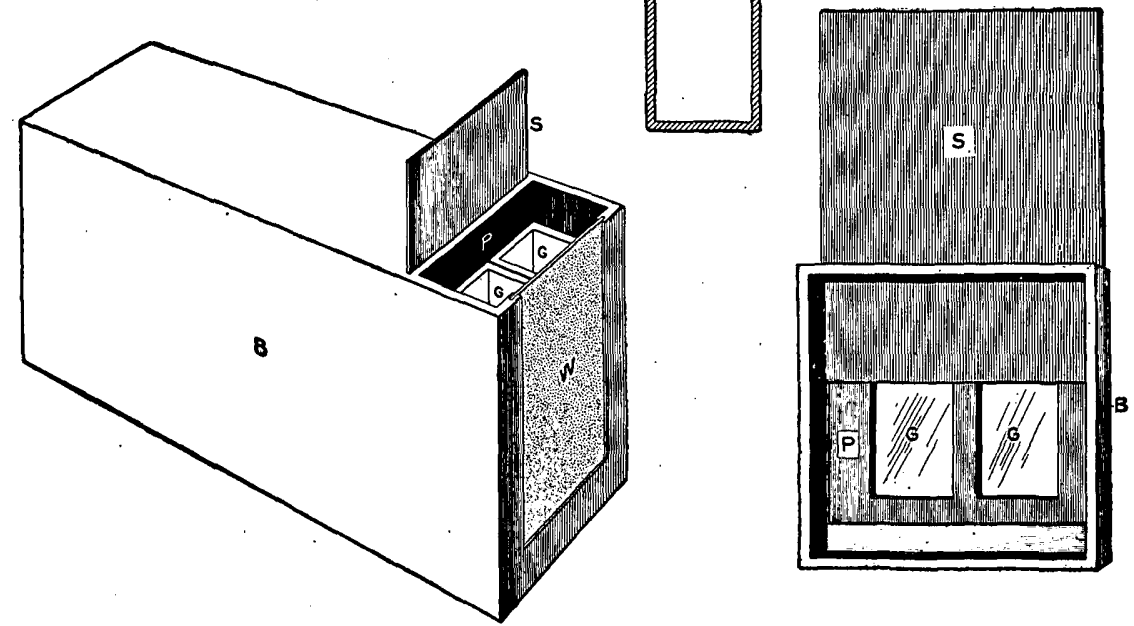

Fig. 5.-First Survey form of apparatus for colorimetric determinations, different aspects. G, One of two glasses of square or rectangular section, 8 to $12 \mathrm{~cm}$. high and 3 to $3 \frac{1}{2} \mathrm{~cm}$. inside measurement between those sides through which the liquid is to be observed. The other sides are blackened on the outside. B, Rectangular box about $35 \mathrm{~cm}$. long and $12 \mathrm{~cm}$. square, stained black inside and out, one end closed by a ground-glass window, $W$, the other open, and a portion of the top removed. $P$, Blackened partition, with openings corresponding to the interior dimensions of the glasses when in position. S, Blackened cardboard shutter sliding stiffly up and down back of the partiticn.

The manner of using the colorimeter is as follows: Any suitable amount of the standard solution, which is purposely made stronger than the solution to be tested, is placed in one of the glasses and an indefinite part or the whole of the test solution in the other. Water is then added from a burette to the standard until there is no distinction as to color. Perfect mixture is brought about by a glass rod flattened at one end. In making the color comparison the box is best held close to a window, so as to get a full, strong light. Daylight is far preferable to artificial light. The amounts of the substance to be determined and that in the standard cylinder are of course proportional to the volumes of the matched solutions. 
b. SECOND SURVEY FORM. (STEIGER's.)

The colorimeter shown in fig. 6 was devised by George Steiger. Though more elaborate in its construction than the one just described, it permits making any number of readings with the same standard solution and has given Mr. Steiger excellent service. Its construction and use are thus described by himself. ${ }^{a}$.

Instruments using the principle upon which this one is based-the ratio of the thickness of the liquid through, and not the actual dilution to equal concentrationsare not applicable to al: colorimetric determinations. It will be found, in comparing

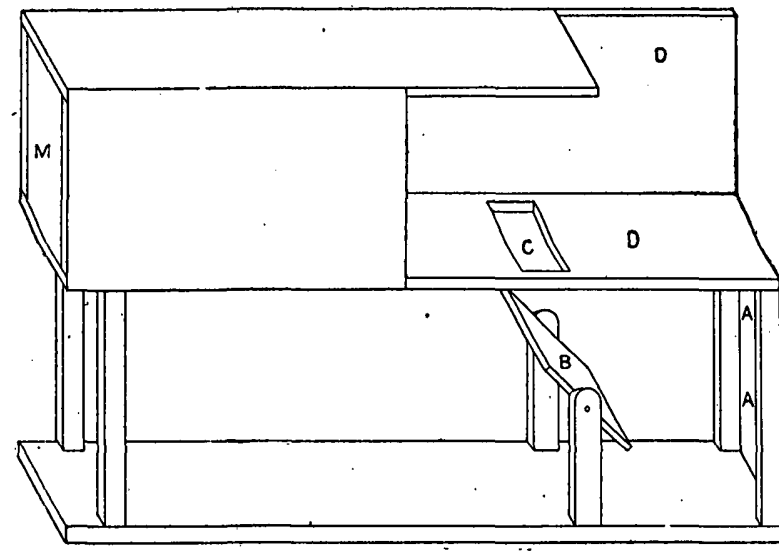

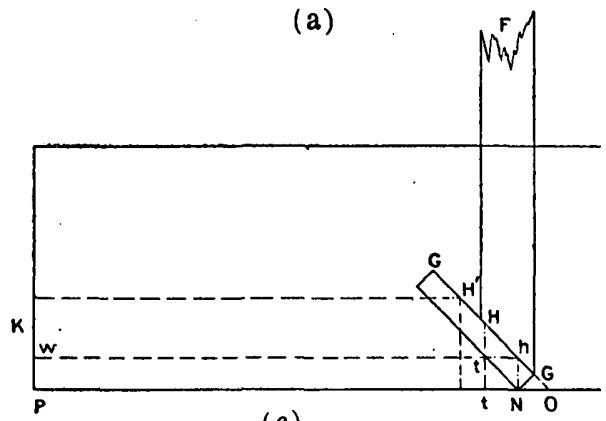

(c)

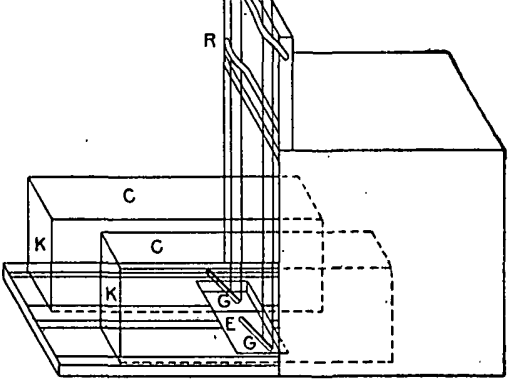

(b)

Fic. 6.-Steiger's colorimeter (second Survey form).

such a solution as is used in the colorimetric determination of manganese and some other substances, that there is a change not only of the intensity of the coler, but also of the color itself, making it impossible to find a point at which two solutions of different concentrations will have the same depth of tint. In some other cases, as for instance the yellow color of the higher titanium salts, this principle gives perfect satisfaction:

The instrument to be described consists of two wooden boxes, the interior portions of which are finished in dead black. In fig. 6 (a), AA is a piece of finely ground glass, and this should be illuminated with the full light of the sky. B is a mirror mounted to swing so that light may be thrown perpendicularly through the hole $\mathrm{C}$.

The second portion of the apparatus consists of a box, as shown in fig. 6 (b), made with two parallel grooves in the bottom, in which the two glass cells $\mathrm{CC}$ can be moved back and forth, and the hole $\mathrm{E}$, which admits light reflected by the mirror $\mathrm{B}$ of fig. 6 (a). 
These cells are about $15 \mathrm{~cm}$. long, $2.5 \mathrm{~cm}$. wide, and $5 \mathrm{~cm}$. deep. On the bottom of each cell and near the outside edge is engraved a scale, a convenient unit for which is the millimeter. FF are glass tubes with mirrors, GG, attached to the lower ends at an angle of $45^{\circ}$. These tubes may be lifted up when it is desired to remove the cells; they may also be removed entirely from the clips RR for cleaning purposes, but they should be pushed down when in use so that the lower edges of the mirrors touch the bottoms of the cells. When ready for use this box is placed in the space marked DD, fig. 6 (a). Care should be taken to place the mirrors at an exact angle of $45^{\circ}$.

Under these conditions, in each cell, all light coming through the bottom of the cell and reflected through the end $\mathrm{K}$ will go through the same thickness of liquid, and if the mirror were a reflecting surface coming in direct contact with the liquid this distance would be represented by the line OP, fig. 6 (c). There is a small error here, due to the converging of the rays to the eye; this is so slight; however, as not to cause any perceptible uneven illumination.

The mirrors being made of ordinary looking glass, the reflecting surface will be the upper side GHG, fig. 6 (c), and the light must go through the glass of the mirror before striking the reflecting surface, and the same on leaving. The distance which the light travels through the glass of the mirror will be represented by twice the length of the hypotenuse of an isosceles right-angle triangle, the equal sides of which are each equal to the thickness of the glass, and must be deducted from the length OP.

A ray of light entering the glass at the point marked $\mathrm{N}$ will travel to $h$ and then be reflected to $t$. From $t$ to $w$ it will go through the colorimetric solution, and this distance is therefore the length to be measured. A point, $\mathrm{H}$, is marked on the mirror near the outer edge, so that it may be seen in the same line of vision as the scale on the bottom of the cell, and perpendicularly above $t$. In looking through the end $\mathbf{K}$, this mark will be recorded at the point $t^{\prime}$ directly below it on the scale, and $t^{\prime} \mathrm{P}$ being the same at $t w$, the distance desired can be read off. The position of the point $H$ is determined by measuring off, on the back of the mirror, a distance from the lower edge equivalent to three times the thickness of the glass. It may be convenient, if thin looking glass has been used, to have this point farther up on the mirror $\left(\mathrm{H}^{\prime}\right)$, in order that it may be seen more plainly, but if so moved an addition must be made to the observed reading equivalent to one of the sides adjacent to the right angle of an isosceles right-angle triangle, the hypotenuse of which is equal to the distance this point has been removed from $\mathrm{H}$. It is convenient in making the graduation on the cell to allow for this correction. The reading can then be made directly.

Glass cells to answer the purpose may be had of any of the large supply hosues, but not graduated; the graduation must be done in the laboratory. The supports FF can be made of rather heavy walled glass tubing, about $1 \mathrm{~cm}$. outside diameter.

The mirrors are made of a good grade of looking glass, the lower and top edges blackened, and cemented to the ground ends of the glass tubes with Canada balsam, after which the backs are coated with paraffin. Paraffin answers well as a coating for a large number of colorimetric solutions. In case a liquid is to be used which attacks paraffin, a substitute must be employed which is unaffected by the liquid in question. It will be found necessary to replace the mirrors from time to time, as it is not possible to so protect the silvered surfaces as to prevent the gradual eating in from the edges by the various solutions used.

The comparison is made by pouring a solution of known strength into one of the cells. The unknown solution made up to a definite volume is put in to the other. The left-hand cell is then placed at a convenient point, which should be determined by the depth of color of the solution it contains. The right-hand cell is then moved back and forth till, on looking in the end M of the apparatus, fig. 6 (a), the two mirrors appear to be of the same shade.

The strengths of the two colorimetric solutions being inversely proportional to the thickness of the liquids looked through, by substituting in the following equation the amount of the material to be determined may be found. 
Let $R$ equal the reading of the cell containing the known solution with a concentration $\mathrm{C}$, and $r$ the reading of the cell containing the unknown solution, which has a concentration $c$, then

$$
c=\frac{\mathrm{RC}}{r}
$$

\section{SCHREINER'S FORM.}

Dr. Oswald Schreiner, of the United States Department of Agriculture, has devised a colorimeter (fig. 7 ) ${ }^{a}$ which has done good service in colorimetric work on phosphates, etc. Its description, personally communicated by Doctor Schreiner, is as follows:

The colorimeter in its simplest form consists essentially of graduated glass tubes $\mathrm{B}$, containing the standard and the unknown colorimetric solution, the column of the liquid in both tubes being changed by means of two smaller immersion tubes $\mathrm{A}$, also of glass. The upper ends of the tubes A are mounted in blocks of wood which fit into grooves on the body of the colorimeter. This permits the ready removal of the tubes from the colorimeter for cleaning when the liquids are changed. The graduated tubes B are supported by a block of wood about midway of the camera and are held in place by brass springs or clamps $\mathrm{C}$, the tension of which can be regulated to suit the diameter of the tube, so as to allow it to be moved freely up or down by hand and yet be firmly held in position when the setting is made. It will be noticed from the figure that the glass tubes diverge slightly. This has been found of advantage, as it allows the operator to look down the center of both immersion tubes, as well. as to bring the upper parts of the tubes, and consequently the images, closer together. The openings to the immersion tubes can be covered by means of a microscope slide to prevent dust from falling into the tubes. 'The tubes are illuminated from the reflector $\mathrm{D}$, below, which carries a white sheet of cardboard or opal glass, and after passing through the tubes the light is reflected by a mirror, $\mathrm{E}$, in the upper part of the instru-

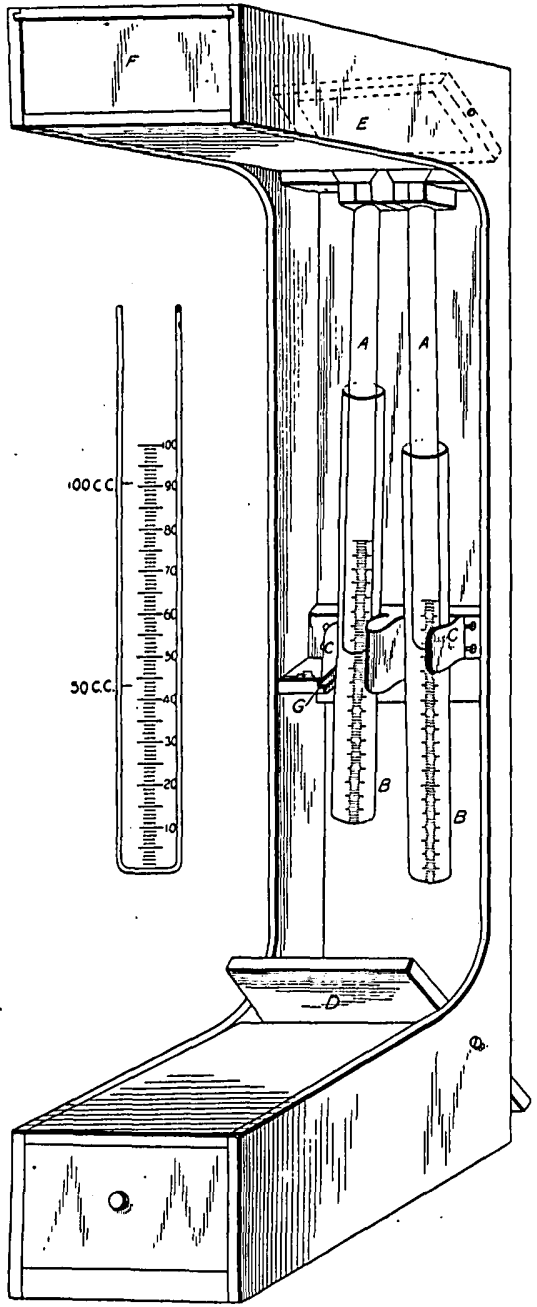

Fig. 7.-Schreiner's colorimeter. ment to the eye of the observer at $F$. The dimensions of the camera are about 70 by 32 by $16 \mathrm{~cm}$. Standard glass slides may be inserted at $\mathrm{G}$ below one of the immersion tubes in place of the tube containing the standard solution, the immersion tute being retained so as to give similar images when viewed from above. 
The measuring colorimeter tubes, $\mathrm{B}$, are $25 \mathrm{~cm}$. long, with inside diameter as nearly as practicable $27 \mathrm{~mm}$.; in no case less than $25 \mathrm{~mm}$. and not greater than $29 \mathrm{~mm}$. The glass should be colorless and the bottoms well ground and polished, with internal surfaces of the bottoms plane, in no case appreciably convex or concave. The bottoms must be ground down sufficiently to make the ground surfaces a little larger than the internal diameters of the tubes; each of the tubes is provided with an etched scale of one hundred 2-mm. divisions. The scale begins at the level of the inner surface at the bottom, the length of the marks being $6 \mathrm{~mm}$. and every fifth mark $12 \mathrm{~mm}$., and every tenth mark numbered on the right side, 10, 20,30, etc., beginning at the bottom. On the reverse side of each measuring tube may be etched two capacity marks, if desired, one for $50 \mathrm{~cm}^{3}$, the other for $100 \mathrm{~cm} .{ }^{3}$.

The smaller tubes are likewise $25 \mathrm{~cm}$. long, with outside diameter not greater than 20 $\mathrm{mm}$. and not less than $18 \mathrm{~mm}$., with the thickness of the glass the same in all the tubes. The bottoms of these tubes must be carefully ground and polished and the inside of the bottom never sensibly convex or concave. They must be of colorless glass, with the bottoms so ground that the diameter of the ground portion exceeds the internal diameter of the tube. These tubes are to be provided with neither scale nor capacity marks.

The standard colorimetric solution is poured into one of the graduated tubes and put into place in the camera together with the immersion tube. The unknown colorimetric solution, made up to definite volume, is put into the other graduated tube and similarly placed in the instrument. The tube containing the solution of unknown strength is set at a convenient height, say fifty scale divisions, and the other tube containing the standard moved up or down, the operator watching the effect on the image in the mirror through the opening in the front of the camera. By moving the tube so that the image is alternately weaker or stronger than the standard, the setting can be accurately and quickly made. When both images show the same intensity of color, the setting is read by noting the division mark on the graduated tube opposite the ground bottom of the immersion tube. This reading will give the height of column of the standard which exactly corresponds with the intensity of color of the column of the unknown solution. It follows that the strengths of these two colorimetric solutions are inversely as the heights of the columns-that is, as the readings on the two tubes. If $\mathrm{R}$ is the reading of the standard solution of strength $\mathrm{C}$, and $r$ is the reading of the colorimetric solution of unknown strength $c$, then $c=\frac{\mathrm{R}}{r} \mathrm{C}$. The same formula, of course, applies when the standard is fixed and the unknown solution moved up or down until the images are of equal. intensity.

\section{PRELIMINARY QUALITATIVE ANALYSIS.}

A complete qualitative analysis of a rock preceding the quantitative examination is in most cases a sheer waste of time. A few constituents may now and then be specially looked for, but in general time is saved by assuming the presence of most of them and proceeding on that assumption in the quantitative analysis.

This statement must not. be misinterpreted, as has occurred, so as to convey the impression that qualitative analysis receives little consideration in the chemical work of the Survey. For ores, mineral species, waters, etc., it is indispensable, as also in the testing of precipitates and filtrates during the course of quantitative analysis.

The student of qualitative analysis will find in the recent publications of A. A. Noyes, W. C. Bray, and E. B. Spear ${ }^{a}$ a. vast fund

a Jour. Am. Chem. Soc., vol. 28, 1907, p. 137; vol. 30, 1908, p. 481; vol. 31, 1909, p. 611; Chem. Nev's, vol. 95,1907 , pp. 89 , etc.; vol. 98,1908 , pp. 6 , etc. 
of information, such as is to be had nowhere else, relating not only to the making of separations, but, and this is often of greater importance, to the completeness of these separations and to the possible limits of detection. The scope of the work is less restricted than its title might be thought to imply, for such elements as glucinum, uranium, vanadium, titanium, zirconium, and thorium are included.

\section{. 9. QUALITY OF REAGENTS.}

Unquestionably the greatest hindrance to good work in otherwise well-equipped laboratories is the difficulty in securing satisfactory reagents. This fact early became apparent to the Survey chemists, and it is mainly to their strenuous complaints and initiative that the present movement in favor of better quality is due. Much of the glassware on the market is of a very inferior grade and utterly unsuited for analytical work.

All analyses performed in the Survey laboratory have been made with the purest reagents obtainable, either by purchase in the open market or by special preparation on the part of manufacturers or in the laboratory, but the most ceaseless vigilance has at all times to be practiced. As is now generally known, a "C. P." label is no guaranty whatever of the purity of a reagent. Every new purchase must be examined if it is one in which purity is a desideratum. In general, all so-called "C. P." chemicals should at least stand the tests laid down by Krauch. . The "guaranteed" or special reagents, sold at high prices, have been found at times to be worse than those emanating from sources which make no claim to special purity for their goods.

Acids made in this country can be had of a high grade, and these in most cases need no redistillation except for special experiments, but exceptions have been noted.

Hydrofluoric acid had to be always freshly distilled with potassium permanganate until the introduction of ceresin bottles afforded an article sufficiently pure for the most exacting work. Care must be taken to see that no particles of paraffin or ceresin are floating on the acid as it is poured out, and that this is free from traces of hydrochloric acid whenever it is to be used for attacking silicates with a view to determining chlorine (C. b, p. 184). An important use for hydrofluoric acid is the decomposition of refractory silicates in the determination of ferrous iron. For this purpose it is necessary that it be free from any substance that will reduce permanganate. Sulphurous acid was found in one or two shipments, but this is a contamination against which care in manufacture can easily guard.

a Die Prüfung der chemischen Reagentien, 3d ed., Berlin, Julius Springer, 1896. English translation by .T. A. Williamson, D. Van Nostrand Co., 1902. 
Ammonia ought always to be redistilled at short intervals, after first shaking up with slaked lime in order to decompose any ammonium carbonate that it may hold. Glass stock bottles may be coated inside with ceresin so as to prevent contact between the glass and the ammoniacal solution, a device that may be serviceable with other reagents.

Potassium bisulphate should be entirely free from silica, alumina, and heavy metals. The only correct way to get an accurate silica determination is to fuse the salt with a considerable amount of strong sulphuric acid, allow to cool, dissolve in water, and digest on the steam bath to coagulate the silica, which is then filtered; or, the salt may be dissolved in water and evaporated with a large excess of sulphuric acid till fumes of the latter rise copiously. On cooling, the mass is dissolved and treated as above. If a satisfactory article is not to be purchased, it can be made from equivalent parts of potassium sulphate and sulphuric acid. Even then the normal salt has first to be examined, for it has been found to contain notable amounts of lead, calcium, and silica. Sodium bisulphate is for some purposes preferable to the potassium salt. Both should, however, be converted to pyrosulphate by fusing in a large platinum dish and maintaining in fusion till all spattering has ceased and sulphuric acid rises copiously. The salt thus treated will not show the disagreeable frothing and spattering that is so objectionable when making fusions with the bisulphate and it is more quickly effective.

The phosphorus salt used for precipitating magnesium has been found to contain iron and silicon.

Ammonium oxalate almost always contains calcium, and must therefore be purified or specially prepared. The same may be said of oxalic acid. According to A. Classen, ${ }^{a}$ ammonium oxalate sometimes contains lead.

Ammonium chloride has been known to contain manganese.

Hydrogen peroxide sometimes contains fluorine, which renders it unfit for use as a chemical reagent. The test for fluorine is carried out as follows: To $50 \mathrm{~cm}^{3}$ of the reagent add a slight excess of sodium carbonate and warm the solution. Filter if a precipitate forms, and to the boiling solution add calcium chloride in excess. Filter and gently ignite the precipitate. Treat it with dilute acetic acid, drop by drop, till the calcium carbonate is dissolved, filter, wash, gently ignite the residue, and test it with strong sulphuric acid in the usual way for fluorine. The perhydrol of E. Merck, now on the market, is a very pure and strong peroxide.

Sodium carbonate, one of the most important reagents in silicate analysis, sometimes can be had which does not carry more than $2 \frac{1}{2} \mathrm{mg}$. 
of total impurity (see $\beta$, p. 88 ) in 20 grams (0.012 per cent). This should be reserved for the main portions, in which silica, alumina, etc., are to be determined. For other constituents, as phosphoric acid, fluorine, sulphur, a poorer grade is quite allowable, provided it is free from the element to be determined and from any other which might interfere with its determination. Silica is to be tested for by evaporating the solution of the carbonate or the dry salt with sulphuric acid in large excess till fumes of the acid are copiously evolved, dissolving the cooled mass in water, and digesting on the steam bath in order to coagulate the silica before filtering. Evaporation with hydrochloric acid will not yield all of the silica. Not long ago a very objectionable impurity in the otherwise acceptable article was encountered, namely fluorine. The explanation of its presence is probably that cryolite had been used as the source of the carbonate. Sodium phosphate in small amounts seems to be a common contaminant of even good grades of sodium carbonate.

Sodium hydroxide made from sodium has been found with a large amount of lead, a fatal obstacle to successful mineral analysis, and also with small amounts of nickel.

Platinum ware may be mentioned here, too, although not a reagent. Recently iron in large amount has been found in shipments fresh from the factory. It may be readily detected by digesting hydrochloric acid in the ware, and sometimes shows on the surface, after ignition, as a red coating.

Owing to the solvent action on glass of many solutions of solid reagents, these solutions should be made up at frequent intervals in limited quantities, or, preferably, the solid should be dissolved as wanted. That this is no unnecessary refinement in really good work has been amply demonstrated, and it is particularly called for with such reagents as ammonium oxalate and microcosmic salt.

\section{PART II.-METHODS APPLICABLE TO SILICATE ROCKS.}

\section{INTROD̃UCTORY REMARKS.}

The order hereinafter followed in describing the various chemical separations has little relation to the affinities of the constituents of the rock, but those are grouped together which can be conveniently determined in the same portion of rock powder. Thus, in the main portion are usually determined $\mathrm{SiO}_{2}, \mathrm{TiO}_{2}, \mathrm{MnO}, \mathrm{NiO}, \mathrm{CaO}, \mathrm{SrO}, \mathrm{MgO}$, total iron, and the combined weight of all the following: $\mathrm{Al}_{2} \mathrm{O}_{3}, \mathrm{TiO}_{2}, \mathrm{P}_{2} \mathrm{O}_{5}$, $\mathrm{ZrO}_{2}$, all iron as $\mathrm{Fe}_{2} \mathrm{O}_{3}$, and nearly if not quite all vanadium, as $\mathrm{V}_{2} \mathrm{O}_{5}$, also perhaps rare earths if present. In a separate portion is estimated $\mathrm{FeO}$, and also the total iron, as well as $\mathrm{BaO}$, if these last are desired as checks. The alkalies need a portion for themselves. In another, $\mathrm{ZrO}_{2}, \mathrm{BaO}$, rare earths, and total sulphur are very conveniently deter- 
mined. For $\mathrm{V}_{2} \mathrm{O}_{3}$ and $\mathrm{Cr}_{2} \mathrm{O}_{3}$ still another and usually much larger portion is to be used. Determinations of $\mathrm{CO}_{2}, \mathrm{C}, \mathrm{H}_{2} \mathrm{O}, \mathrm{Fl}, \mathrm{Cl}$ are all best made in separate portions of substance, though various combinations are possible, as $\mathrm{CO}_{2}$ and $\mathrm{H}_{2} \mathrm{O}, \mathrm{C}$ and $\mathrm{H}_{2} \mathrm{O}$, or $\mathrm{H}_{2} \mathrm{O}, \mathrm{Fl}$, and $\mathrm{Cl}$. In fact, by a judicious selection and combination of methods a very satisfactory analysis can sometimes be made on 4 grams. of material without omission of anything of importance, though the time consumed will be greater than if ample material is available.

As an illustration of the advantage to be gained by a little judgment in the combination of methods, the case of sulphur, barium, zirconium, and rare earths may serve. Many chemists never look for any but the first of these, but by following the procedure given under A, page 138, not much more labor is expended in confirming their presence or absence than that of sulphur alone.

With only occasional exceptions nearly all the constituents mentioned on page 23 can be determined if present in portions of powder not exceeding 1 gram each in weight.

This is a convenient weight to take for the main portion in which silica, alumina, etc., the alkaline earths, and magnesia are to be sought; but it should, in general, be a maximum, because if larger the precipitate of alumina, etc., is apt to be unwieldy. Its weight can not often be much'reduced with safety if satisfactory determinations of manganese, nickel, and strontium are to be expected. For the alkali portion one-half gram is a very convenient weight. In general, it may be made a rule not to use more than 2 grams for any portion which has to be fused with an alkali carbonate, as for sulphur, fluorine, and chlorine. For carbon dioxide the weight may rise to 5 grams, or even more, if the amount of this constituent is very small, without expenditure of any more time than is required by $1 \mathrm{gram}$ and with correspondingly greater approach to correctness in the result. For vanadium also a larger weight than 2 grams is usually demanded.

For the attainment of accurate results, such as are needed for the close classification of a rock in the system devised by Cross, Iddings, Pirsson, and Washington, ${ }^{a}$ too great stress can not be laid on the exercise of the utmost care throughout the analysis. This applies not only to routine manipulations, but even more in the matter of double precipitations, where these are called for, and in the testing of filtrates and precipitates to insure complete recovery of the desired substances, on the one hand, and freedom of them when obtained from matter which should not to be there, on the other. These cautions are especially urgent in the case of those having little experience. It must always be remembered that analysis can at best afford but approximate results, which will be more serviceable the greater the care bestowed in obtaining them. 
- GENERAL REMARKS.

Though a knowledge of the specific gravity of rocks is seemingly less prized now than formerly by petrographers, it is occasionally needed and is ever a most important criterion in the identification of mineral species. As ordinarily carried out, whether by the method of suspension or by the pycnometer, the results fall short of the accuracy which is generally credited to them. A really accurate determination of the specific gravity of solids by the pycnometer, in which the error shall not exceed one or two points in the third decimal, is to be achieved only with the most painstaking precautions (see B. b, p. 48). Without this care the error in the second decimal may, and often does, amount to several points. For these reasons the subject of specific gravity is treated in some fullness. Although the porosity of a rock is a constant seldom used by the petrographer, it is one that is most useful in the comparative study of building stones; and since its determination as often carried out is likewise faulty and gives a value which does not represent the pore space in terms of the volume of stone, it has been deemed expedient to devote space to this subject also, especially as the determination of porosity involves that of specific gravity.

\section{A. BY SUSPENSION IN WATER.}

a. ORDINARY METHOD FOR SPECIFIC GRAVITY.

This determination; when required, is best made on one or several fragments weighing up to 20 grams. They are held together by a fine platinum wire ready for suspension from the balance, and thus held are placed in a small beaker to soak over night in distilled water under the exhausted receiver of an air pump side by side with a similar beaker of water. Boiling is, of course, a much less effective means of removing air than the air pump, and the boiling water may exert an undesirable solvent and abrading effect. In the morning the wire is attached to the balance arm, the rock fragments remaining immersed in the water; a thermometer is placed in the companion beaker of water, now likewise in the balance case, and the weight is at once taken. Both vessels of water having precisely the same temperature, it is quite unnecessary to wait for the water to assume that of the balance should it not already possess it. The fragments are now lifted out, without touching the vessel, and carefully transferred to a tared crucible or dish. The wire is removed and at once reweighed, with the precaution that it dips just as far into the water now as when weighted. Hereby a special weighing of the wire out of water is avoided. The sample may now be dried on the water bath and then at $110^{\circ}$ for some hours to certainly expel all absorbed water, and 
weighed after prolonged cooling in the desiccator. It is better to ascertain the weight of the dry rock after soaking in water than before, in order to avoid the error due to possible breaking off of a few grains between the two weighings. Should the density of the rock in air-dry condition be required, it may be left exposed to the air for a long period after drying and before weighing; ${ }^{a}$ but the difference will only in exceptional cases affect the second decimal by more than a single unit. For instance, an undried rock of 2.775 specific gravity containing in the uncrushed state the high percentage of 0.3 hygroscopic moisture will have a density of 2.79 when dry; a rock of 2.982 specific gravity, undried, will have a density of 3.00 after removal of 0.3 per cent of moisture. The difference becomes greater as the density of the rock increases.

This method of ascertaining the specific gravity of rocks is certainly more convenient than, and for compact rocks is believed to be decidedly preferable to, that of the pycnometer, in which the fragments must be reduced to small size with consequent formation of more or less powder, which is subject to slight loss in the various manipulations. To exclude this powder and employ only small fragments would introduce a possible source of error, since it is likely to consist largely of the most easily abraded minerals and consequently not to have the average composition of the mass. By following the instructions given above, loss of material is absolutely avoided, a decided saving in time is effected, and considerable weights can be easily employed with consequent lower probable error in the results. To vesicular rocks, however, notably certain lavas, the above pro-

$a$ In view of the uncertainty as to what constitutes hygroscopic water (see pp. 58-59), this course is perhaps more to be-commended than the former, and seems imperative for certain zeolitic rocks. In such cases it is best to weigh the rragments before putting to soak, and afterwards to collect on a Gooch crucible the grains which may have fallen off in the water. Should no crucible of this kind be available, a paper filter may unhesitatingly be used and incinerated with the powder, owing to the small amount of which last the error due to loss of even all its water during ignition is quite negligible.

Since the first publication of the foregoing in Bulletin No. 148, a series of determinations on eight rocks, in which the weighing was done after drying led to an interesting observation. The lumps were heated for a short time on the steam bath until dry on the surface, then allowed to cool in air and weighed from time to time during two or three days to constant weight. It was observed that some lost weight from the start, while others gained. For instance:

$\begin{array}{rrr}14.3803 & 9.5342 & 17.0846 \\ .3730 & .5178 & .1089 \\ .3666 & .5121 & .1159 \\ .3483 & .5024 & .1413 \\ .3481 & .5016 & .1388 \\ .3450 & .5016 & .1379 \\ .3457 & & \end{array}$

The losses in the first and second cases may reasonably be ascribed to the gradual evaporation of water absorbed in the pores, while the increase in thc third case is doubtless indicative of the presence of zeolitic constituents, from which even brief exposure to the heat of the steam bath had sufficed to drive out a good deal of their loosely held water. Subsequent careful analysis confirmed this view, for each rock that gained in weight was found to hold a relatively large amount of water removable at $105^{\circ}$, and the microscope revealed the presence of zeolitization in them and not in the others. Hence, this procedure may be sometimes of value as a test for the presence of zeolitic minerals in advance of or as confirmation of the microscopic diagnosis. 
cedure is, of course, inapplicable, since it is probable that many of the interior pores would be inaccessible to penetrating water.

b. METHOD FOR SPECIFIC GRAVITY AND POROSITY COMBINED.

E. R. Buckley ${ }^{a}$ has called deserved attention to the faultiness of the methods generally employed for the determinations of these constants as applied to building stones, and has brought together in the work cited the fruits of his experience. His recommendations are essentially as follows:

The specimens should be cubes, such as are commonly employed for the other physical tests, measuring usually 2 inches square. These are dried for at least twenty-four hours at $110^{\circ}\left(100^{\circ}\right.$ is not high enough to insure the removal of all interstitial water) and weighed to centigrams. They are then placed on the bottom of a large bottle, the cork of which can be hermetically sealed. Through the cork pass three tubes, two of which end below the cork and connect with a vacuum pump and manometer, respectively. The third tube, provided with a stopcock, ends near the bottom of the bottle and leads into a basin of water which can be heated to boiling. The bottle itself rests in a water bath, so that its contents can be kept hot all the time. At first the cubes rest in a shallow layer of water only, the better to facilitate the escape of air and entrance of water under reduced pressure, which should not exceed one-twelfth of an atmosphere. The cubes should remain in this state for seventy-two hours, experiment having shown that little or no absorption takes place after this lapse of time. Water is slowly let in from time to time, till the cubes are wholly immersed. In this way it is thought that thorough saturation can be attained. The saturated samples are transferred to an open dish filled with water and taken to the balance, where their weight in water is ascertained by suspension. They are then removed from the water, rapidly dried on the surface with bibulous paper, and the weights then taken in air. ${ }^{b}$ They are dried at $110^{\circ}$ for twentyfour hours and again weighed. The second dry weight was found by Doctor Buckley to vary rarely from the first by as much as 0.2 gram, and often by not more than 0.01 . "When reckoned in per cent, the error would. be such a small fraction of 1 per cent as not to be

$a$ Building and ornamental stones of Wisconsin: Bull. Wisconsin Geol. Nat. Hist. Survey No. 4, 1898, pp. 63, 70.

$b$ The above procedure differs from that of Doctor Buckley in the inversion of the order of weighing in water and after wiping. The more perfect the vacuum the lese need be the temperature of the bath of immersion to insure the effective aid, by boiling of the water drawn up into the pores of the specimen, in securing expulsion of the inclosed air. A vacuum desiccator can perhaps be substituted for the bottle with much advantage. It will not be necessary to open the desiccator for the periodic introduction of water if to the evacuating tube is attached a $Y$ or $T$ with stopcocks, one branch leading to the pump and manometer, the other to a basin of water. By proper regulation of the cocks water can be drawn in at pleasure. In a later publication (Missouri Bureau Geol. and Mines, 2d ser., vol. 2, 1904) Doctor Buckley states that he has reduced the time of immersion to twenty-four hours and the vacuum to one-fourth of an atmosphere, without finding appreciable differences in the results obtained. 
worthy of attention. The specific gravity was determined by dividing the average of the two dry weights by the difference between the dry weight and the weight of the cube suspended in water." For arriving at the true porosity or actual pore space compared with the volume of the sample tested, the following formula is to be used:

$$
\frac{(B-A) \text { sp. gr. } 100}{(B-A) \text { sp. gr. }+A}=P
$$

in which $A$ is the average dry weight, $B$ the saturated weight, and $P$ the percentage of porosity or actual pore space. The value thus obtained is always much higher than that ordinarily taken as the measure of porosity. This last would better be called, according to Doctor Buckley, "ratio of absorption," being found by dividing the weight of water absorbed by the drý weight of the stone and multiplying by 100 . Doctor Buckley finds no definite relation between porosity and ratio of absorption, although in a general way the former is a little more than twice the latter:

In calculating the weight of a stone in pounds per cubic foot the pores should be included in this volume. The weight sought is found by multiplying the apparent specific gravity by 62.5 -that is, by the weight of a cubic foot of water. The apparent specific gravity can be calculated from the above data by the formula

$$
\frac{\mathrm{A}}{\mathrm{B}-\mathrm{C}} \text {, }
$$

in which $\mathrm{A}$ is the dry weight, $\mathrm{B}$ the saturated weight, and $\mathrm{C}$ the weight in water.

T. W. Thörner ${ }^{a}$ and H. Seger and E. Cramer ${ }^{b}$ have described apparatus alike in principle for determining porosity by the use of which a direct specific-gravity determination is avoided and the operations are very simple. The apparatus of Seger and Cramer are of simpler construction than those of Thörner, and have stood the test of many years' use. By both, however, the true porosity as well as the "ratio of absorption". is ascertainable.

C. PENFIELD'S METHOD FOR MINERAL FRAGMENTS.

Penfield ${ }^{c}$ recommends the following modification of the suspension method as more convenient than that by the pycnometer in many cases for small fragments of minerals.

After boiling in water, the substance is transferred with water to a small glass tube about 8 by $35 \mathrm{~mm}$., provided with a fine platinum wire for suspension. This is weighed full of water in another vessel of water, and again after the removal of the mineral, the weight of which is found after drying. 
This method is, of course, more applicable to homogeneous minerals than to rock fragments, and will therefore be applied in rock analysis chiefly to the determination of the specific gravity of the mineral grains separated by heavy solutions or acids.

\section{B. PYCNOMETER METHOD.}

a. ORDINARY METHOD.

If the pycnometer has to be used, as is generally the case when the density of any one of the mineral ingredients of a rock is desired after separation by one of the approved methods, it being then in a more or less finely divided state, a good procedure is that adopted in this laboratory by L. G. Eakins a number of years ago, provided there is no evaporation whatsoever around the stopper, a condition seldom, if ever, fulfilled, unless a thin film of vaseline is used, the weight of which need not exceed a half milligram. ${ }^{\checkmark}$ The pycnometer used is one with a capillary stopper, provided with a millimeter scale etched in the glass, the divisions being numbered both ways from the center and calibrated by mercury, so that the value of each one in weight of water is known. The capacity of the flask filled with water to the zero division is then calculated for every half degree of temperature from $0^{\circ}$ to $30^{\circ}$ by making a series of careful weighings in which, the capacity of the stem being known, it is quite immaterial at what level the water stands, provided it is within the limits of the scale. The exact temperature is obtained by an accurate thermometer placed in a companion vessel of similar shape to the pycnometer and containing a like amount of water, both being left in the balance case till its temperature has been nearly or quite assumed, as shown by a second thermometer. The weighing must of course be made before the thread of water has sunk beneath the lowest division, which it will do after a time, even though at first filling the bore to the top of the stopper; and the corrected weight full of water to the zero mark is found by adding or subtracting the needed amount, as shown by the height of the thread on the scale.

For each pycnometer in use, and these are of different sizes, is prepared a table showing its weight, the value of each scale division in grams of water, and the capacity of the flask at different temperatures, as indicated above. The preparation of such a series of flasks is time saved in the end, for the weighing of the flask full of water each time a density determination is made is rendered superfluous. All that is necessary is to look up in the table the weight corresponding to the temperature.

- The density of the previously weighed substance in this case is now determined in much the same way as in A. a, page 43 , after the unstoppered pycnometer containing it and nearly filled with water has stood 
with its companion vessel of water under the air pump the necessary length of time. The water needed to fill the flask is taken from its companion.

All who have used the pycnometer method for fine substances know the difficulty experienced in preventing a portion from being held at the surface, despite all attempts at making it sink. Hence it often happens that a very small portion runs out around the sides of the stopper on inserting it. If the flask rests in a small tared dish the grains thus forced out may be washed down into it and weighed after evaporation in order to get the correct weight of that in the flask; or, after weighing, the contents of the ftask may be emptied into a tared dish and the water slowly evaporated off in order to get the weight of the mineral. Usually this way is less to be recommended than the other.

\section{b. REFINED METHOD.}

In their elaborate study of the thermal properties of the feldspars ${ }^{a}$ Drs. A. L. Day and E. T. Allen found the ordinary methods of specific-gravity determination of powders by the pycnometer to be affected with grave errors. In the following is given their procedure, slightly modified since publication, for obviating them to such a degree that with $25 \mathrm{~cm} .^{3}$ pycnometers and 5 to 10 grams of material of from 2.50 to 2.75 specific gravity the error from all causes should never be greater than one unit $( \pm 1)$ in the third decimal place.

A chief source of error is the evaporation of water around the stopper. In order to reduce this to a negligible quantity, the grinding should be very fine-and the stopper should be slightly vaselined before the final weighing. If the excess of vaseline is carefully wiped off, the error due to its weight will not affect the result in the third decimal with $25 \mathrm{~cm} .^{3}$ flasks. The best flasks are those with capillary stoppers, and Day and Allen now have them made with necks $2 \mathrm{~mm}$. thick, so as to avoid springing when the stopper is pressed in, and ground square off to prevent the adhesion of a film of .water between stopper and neck above the joint. With the stopper slightly vaselined, such a flask does not lose at $30^{\circ}$ more than 1 to $1 \frac{1}{2}$ milligrams per half hour.

To obviate another serious source of error, incomplete removal of air from the powder and undue loss of powder during filling and stoppering, the device of G. E. Moore ${ }^{b}$ is made use of, slightly modified, as shown in fig. 8. The flask containing the powder is attached to a water pump, with the intervention of a tube and bulb attachment, as shown. The bulb A contains boiled water. When the system has been nearly freed from air by the pump, water from $A$ is made to flow into the flask. Then by tapping and plunging the latter into water

$a$ Pub. Carnegie Institution of Washington, No. 31; and in less detail in Am. Jour. Sci., 4th ser., vol. 19, 1905, p. 93; Zeitschr. phys. Chemie, vol. 54,1905, p. 1 .

b Am. Jour. Sci., 3d ser., vol. 3, 1872, p. 41; Jour, prakt. Chemie, vol. 2, 1870, p. 329. 
warmed to $40^{\circ}$ to $50^{\circ}$, in order to produce boiling witnin the flask, the air is soon removed. During the boiling powder will usually be thrown up into the connecting tube; but this can be washed back by water from A or, after disconnecting, by boiled water from a wash bottle. Should any particles remain in the tube, they are washed into a tared dish and weighed, after evaporation of the water. ' It is absolutely essential that not the smallest grain be caught between the stopper and the neck. To this end the neck is wiped out with a bit of filter paper, which is burned in the tared dish in order to obtain the proper correction.

Constant temperature is attained in a water thermostat electrically regulated to tenths of a degree at a temperature slightly above that of the balance case. In the thermostat there is also placed a small vessel with water for the final filling. If the powder is very fine, the flask

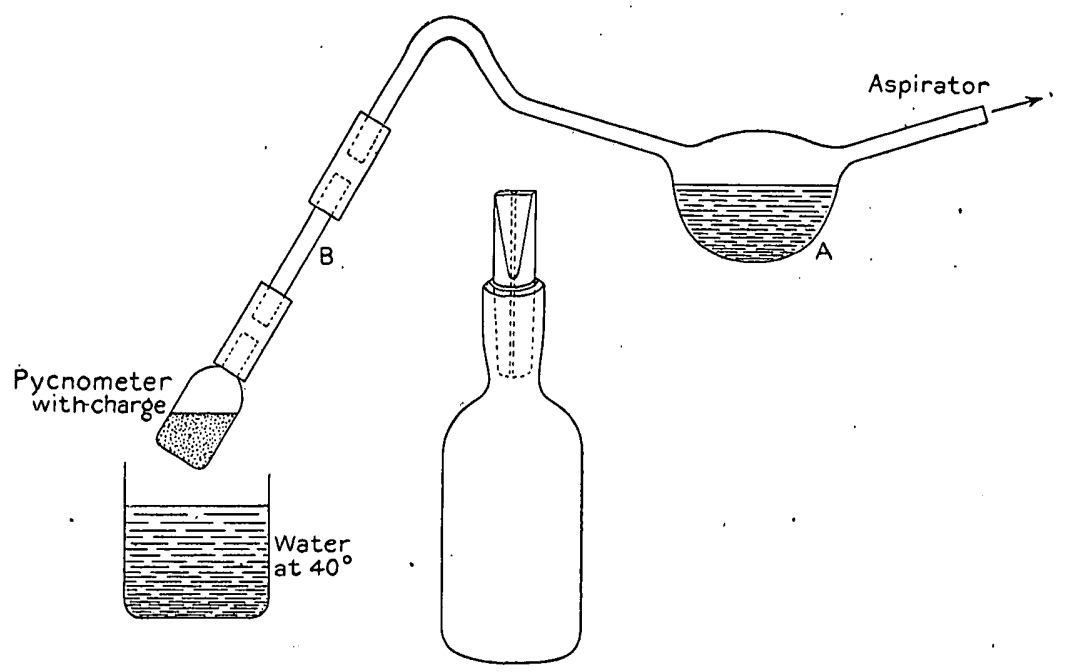

FIG. S.-Moore's device to remove air from mineral powders.

may have to remain in the thermostat several hours to allow complete settling before wiping the neck, vaselining, and stoppering. When filled and stoppered, it is advisable to return it to the thermostat for a time, with a short piece of rubber tube slipped part way over the end of the stopper, so as to form a cup for a drop of water and insure that the capillary remains filled. At the proper time the rubber is removed and the flask quickly wiped with bibulous paper and weighed without delay. A blank test must of course be made with water alone, or the pycnometer may be calibrated once for all within any desired range of temperature. A determination of the accuracy attainable in this way is subject to a correction for buoyancy.

(See p. 64 for remarks by Doctors Day and Allen on another source of error in the determination of the specific gravity of powders.)

20091-Bull. 422-10_-4 


\section{HEAVY SOLUTIONS NOT SUITABLE FOR ROCKS.}

Because of their roughness, porosity, and complex mineral composition the density of rock fragments can not be accurately determined by that of heavy solutions in which they may remain suspended.

\section{PREPARATION OF SAMPLE FOR ANALYSIS.}

\section{A. QUANTITY OF ROCK TO BE CRUSHED.}

In the great majority of cases a few chips from a hand specimen will well represent the average of the mass, but with rocks in which a porphyritic structure is strongly developed the case is different. Here a large sample should be provided, gaged according to the size of the crystals, and the whole of this should be crushed and quartered down for the final sample. Unless this is done, it is manifest that the analysis may represent anything but the true average composition of the rock. The geologist should see that a fairly representative sample is provided, else the chemist's work, however careful, may count for little.

\section{B. CRUShing.}

For accurate analyses the use of steel power crushers and mortars is out of the question because of the danger of contamination by particles of metal and the impossibility of cleansing the roughened surfaces after they have been in use a short time. Extraction, by the aid of a magnet, of steel particles thus introduced into the powder is quite inadmissible, since the rocks themselves, almost without exception, contain magnetic minerals.

\section{a. SURVEY METHODS.}

For breaking large pieces of rock to small sizes a thick steel plate with specially hardened surface and a similarly hardened pounder, such as street pavers use, will probably render the best service, but the hardening must be done with extreme care.

A method of rough crushing on a small scale that gives fair satisfaction in practice is to place each fragment as received on a hard steel plate about $4 \frac{1}{2} \mathrm{~cm}$. thick and $10 \mathrm{~cm}$. square, on which is likewise placed a steel ring $2 \mathrm{~cm}$. high and of about $6 \mathrm{~cm}$. inner diameter, to prevent undue flying of fragments when broken by a hardened hammer. In this. way a considerable sample can soon be sufficiently reduced for transfer to the agate grinding mortar with a minimum of metallic contamination.

This method is now superseded in the Survey laboratory by one devised by C. W. H. Ellis, mechanician in the geophysical laboratory of the Carnegie Institution, which entirely obviates loss of material by flying fragments. The method of operating is apparent from 
fig. 9. The block, cylinder, and pestle are of the best chilled tool steel, surface hardened to the utmost limit. The cylinder fits snugly into a depression in the block, after the manner of an ordinary "diamond" mortar, but the pestle has a diameter less than the inner diameter of the cylinder. The crushing is done by the pestle, without the aid of a hammer, and may be carried so far, if necessary (see C, p. 52), as to furnish by the aid of a fine sieve, preferably of the best silk bolting cloth, a powder that is at once fit for analysis without needing further pulverization in a mortar. For very many rocks and minerals a material that passes a sieve of 30 meshes to the linear centimeter can be used directly for the analysis. Much the greater part of this will consist of grains of a diameter less than that of the mesh of the sieve, even when the intervals between successive applications of the pestle and shiftings are very short.

Metal sieves must on no account be used if search is to be made for elements identical with those of which the sieves are made.

An imperative precaution, when using either of these methods, however, is to refrain absolutely from the least grinding or rubbing motion, with the hammer in the one case or the pestle in the other. Even quartz can be broken down by vertical blows to a size fit for the agate mortar without show-

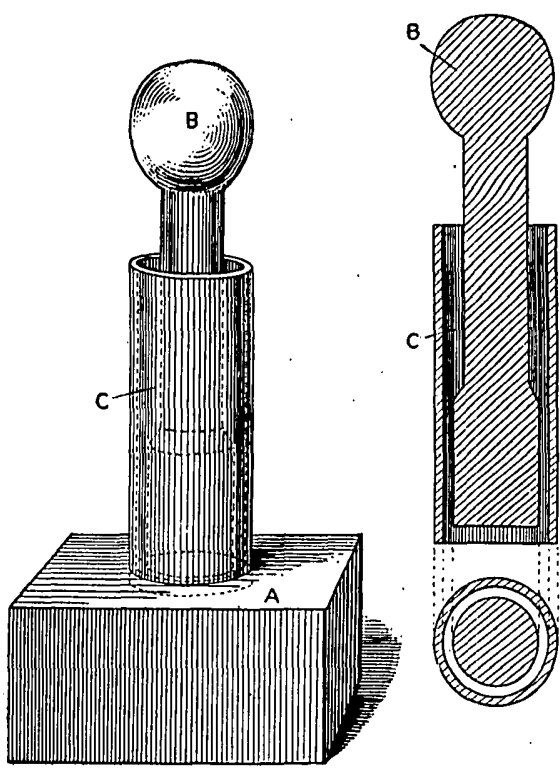

FIG. 9.-Ellis's mortar for crushing coarse materlals. All parts of best chilled and surface-hardened tool steel. A, Block, $12 \frac{1}{2}$ by $12 \frac{1}{2}$ by $6 \mathrm{~cm}$., with depression in center $0.6 \mathrm{~cm}$. deep; $\mathrm{B}$, pestle, $20 \mathrm{~cm}$. high, $3.5 \mathrm{~cm}$. diameter at base; $C$, cylinder, $12 \frac{1}{2} \mathrm{~cm}$. high, $5 \mathrm{~cm}$. outside diameter, $4.4 \mathrm{~cm}$. inside diameter, fitting accurately the depression in the block.

ing any darkening in color, whereas if a rubbing motion has been employed to even a limited extent, the powder will, by contrast, be perceptibly dark after grinding in the mortar. The hardest steel is very susceptible to abrasion by minerals less hard than quartz. ${ }^{a}$ The crushing must be carried to such a degree of fineness that after transferring the grains to the mortar the grinding motion of its pestle

$a$ When the crushing has been done with proper care, the amount of steel contaminating the sample is so small as to cause no important error. This steel is, apparently, for the most part not in splinters but as thin films on the mineral grains. Its presence can be readily shown by grinding the crushed sample to a very fine powder in presence of water. The films thus become separated from the grains and still further flattened, and as the grinding progresses they rise as a visible scum to the surface of the water, whence they can be removed by careful handling of a magnet. This behavior of the films might perhaps afford a means of testing for native iron in rocks, provided the use of steel for crushing could altogether be avoided. 
will not occasion further loss of flying particles, since the portions lost will, in case of all but glassy or very fine-grained material, not have the same composition as those retained.

If grinding is not to follow crushing, the sieve should be chosen of a mesh that will afford a powder susceptible of complete or nearly complete decomposition within twenty minutes by boiling dilute hydrofluoric acid (see C, below, and 19, p. 157). In order to obtain the minimum of "flour" it is important to sift at very frequent intervals. Some flour is, however, essential to the production of a homogeneous sample when flaky minerals, like the micas, are present. If the flour is first separated from a micaceous powder, no amount of mixing of the coarser material will afford a sample in which the mica does not show in streaks instead of being uniformly distributed. The sample finally obtained is to be very thoroughly mixed on glazed paper or by gentle shaking in a clean bottle.

\section{b. WASHINGTON'S METHOD,}

The rock is first reduced, as by the methods in a, to lumps that will fit into a " diamond" steel mortar of Plattner's form, the bottom of the cavity being hemispherical and matched in shape by the end of the pestle. The lumps are crushed, one by one, in the mortar by a dozen blows of a half-pound hammer (billet of wood, Dittrich), and the contents of the mortar are emptied into a cylindrical glass box about $3.5 \mathrm{~cm}$. deep and $7.5 \mathrm{~cm}$. internal diameter, with walls about 2 $\mathrm{mm}$. thick. When the whole sample, or a quartered portion if large, has been thus crushed a piece of best silk bolting cloth with about 25 meshes to the linear centimeter is stretched over the open side of the box and clamped fast by a brass ring $1 \mathrm{~cm}$. in height. The box is now inverted and the finest powder gently sifted onto a sheet of glazed paper. The material left on the cloth is recrushed in the mortar and resifted, these operations being repeated until all has passed the sieve. It will, of course, not do to reject any portion, for thereby the composition of the mass will be affected. The sample is now fine enough to be reduced by grinding in a large agate mortar.

Doctor Washington finds that in this way there is no danger of serious contamination by metallic iron (see preceding footnote) and that any error resulting from introduction of cloth fiber is negligible. This last will hardly be true of the method practiced in parts of Europe (the German "beuteln"), which, at least as it was carried out in Bunsen's laboratory, differs in essential points from the gentle sifting of Doctor Washington.

\section{GRINDING.}

Ordinarily an extremely fine state of division is unnecessary, except in the case of those portions in which alkalies and ferrous iron 
are to be determined, or where soluble constituents are to be removed by acids, etc., and in such cases the final grinding can be done at the balance table on a small portion of the crushed sample.

Not only is fine grinding for most purposes needless, but it may be positively harmful, allowing escape of contained gases, and, as $R$. Mauzelius has recently shown, ${ }^{a}$ it is absolutely imperative for the correct determination of ferrous iron to use the coarsest powder that can be decomposed completely by the method of attack employed. I have since shown ${ }^{b}$ that this is true for the water that is not given off at $100^{\circ}-110^{\circ}$. Mauzelius finds that the finer the material (containing ferrous iron) is ground the less ferrous iron is found. In all his experiments on rocks and on iron-bearing minerals separated from rocks the oxidation was marked and in the most extreme case amounted to about 40 per cent of the total ferrous iron (3.13 per cent $\mathrm{FeO}$ found in the finest powder against 5.13 per cent in the coarsest). This totally unexpected behavior has been confirmed by myself (see 19, p. 154). The necessity for an entire reversal of the hitherto prevalent practice of preparing a very fine powder for the ferrousiron determination is obvious.

The preparation of two samples, one coarse for the ferrous iron and another much finer for the other constituents, though perhaps unavoidable at times, is objectionable for several reasons. (1) The finer the powder the more loosely held (hygroscopic) water it contains, easily 5 to 20 times as much as unground grains, and also the more water that is not given off at $100^{\circ}$. If two samples are used the water given off both below and above $100^{\circ}$ must be determined in each and a correction applied to the other constituent or constituents that may be determined in the finely ground sample. (2). The composition of the unground sample will in another respect differ from that of the ground material, by the amount of silica derived from the mortar. This may easily reach or exceed one-half of 1 per cent of the weight of the mineral powder (see p. 56), depending on the hardness of the minerals and on the length of grinding.

In order to obviate the need for two samples I have made experiments with a view to secure fine grinding and at the same time avoid oxidation. These experiments need to be multiplied before positive conclusions can be reached and precise directions formulated. So far as they go, however, they show that grinding in a nonoxidizing medium, such as absolute alcohol, is fairly satisfactory. Water is somewhat less satisfactory, notwithstanding the lower solubility in

a The determination of ferrous iron in rock analysis: Sveriges Geol. Undersökning, Arsbok 1, 1907, No. 3.

$b$ The influence of fine grinding on the water and ferrous iron content of minerals and rocks: Jour. Am. Chem. Soc., vol. 30, 1908, p. 1120; Chem. News, vol. 98, 1908, pp. 205, 215. 
it of oxygen. ${ }^{a}$ Alcohol does not seem to exercise a reducing effect on ferric minerals when they are ground in it. These experiments have served to show at the same time that the oxidation of the iron is not due merely to the greater exposure of surface, but that it is probably aided by intense local heat from friction and fracture of the grains under the pestle. Probably grinding in an inert gas would be more satisfactory, but while this might be arranged with a mechanical grinder, it would be impossible when the grinding has to be done by hand, and in any case would involve much inconvenience and trouble. When alcohol is used, it should be in small quantities at a time, enough to wet the powder thoroughly and to give a very liquid mass. A second and third application of alcohol are sometimes needed, because of the rather rapid evaporation. When the grinding is finished the alcohol is allowed to evaporate spontaneously under a cover placed a few centimeters above the mortar and pestle, so as to exclude falling dust and yet allow free circulation of air. When thoroughly free from alcohol the powder is removed gently from mortar and pestle with a flexible knife blade and then bottled.

Formerly all rock samples in the Survey laboratory were, after crushing, reduced to powder by hand grinding, with expenditure of much time and labor. Since then, except in special cases, the work has been done by mechanical grinders run by a small electric motor. It will be evident from the foregoing statements, however, that hereafter such grinders are likely to be less used than they have been.

The grinder manufactured by the McKenna Brothers Brass Company (Limited), Pittsburg, Pa., is shown in fig. 10, with the addition of simple attachments intended to keep metal dust and oil out of the mortar. These additions have been found to be quite essential for our work. As made for the technical industries the machine is largely of brass, nickel plated, but the latest made for the Survey is of steel throughout, without plating. It was found that the pulley belts caused too much abrasion of the softer metal to permit its use for much of our work, on account of the danger of copper, zinc, and nickel getting into the sample in traces, and that in spite of a very. limited use of oil this invariably became scattered over the contents of the mortar, though its presence never became visible to the eye except when white materials were ground. The last contamination is prevented by the copper cylinder, A, soldered securely onto the pestle holder so as to form a cup large enough for the purpose intended. The tin shield, B; with a superposed rubber cloth, C, covering the slit required for the proper adjustment of the shield, greatly lessens the danger of metallic contamination, even with the all-steel machine. At first it was thought that a rubber cloth covering the mortar, with the pestle snugly fitting a hole in the cloth, would be an effective pro- 
tection, but it was found that there was sufficient friction on the rubber itself to cause contamination of the powder beneath.

With this apparatus a large amount of work can be done in a short space of time, though there is much loss of fine powder if the speed of

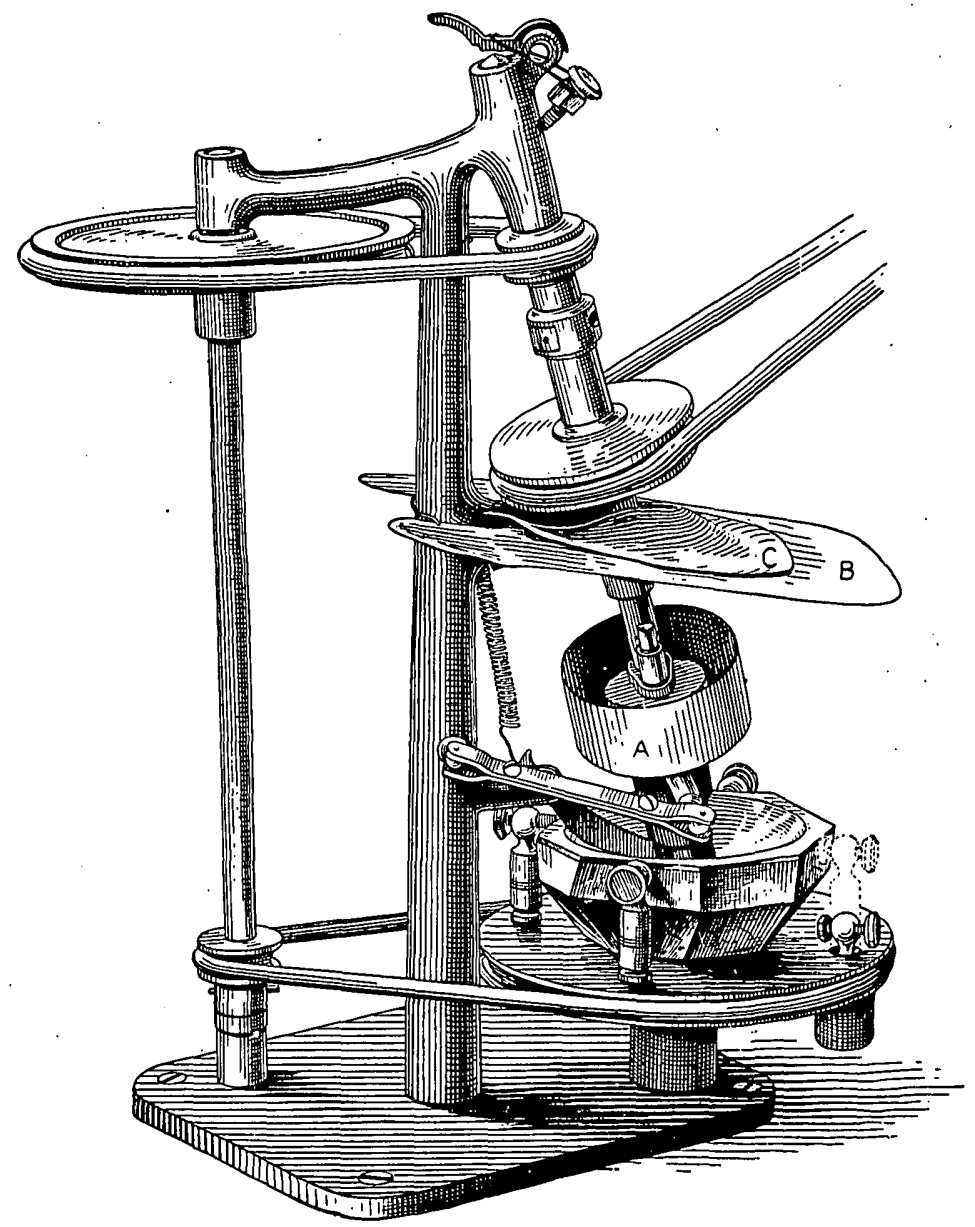

FIG. 10.-McKenna ore grinder, slightly modified by the addition of the following attachments to prevent oil and dust from belts and bearings from entering the mortar. A, Copper cup soldered to head of pestle holder. This partakes, without the slightest friction, of the motion of the latter. B, Tin plate with deep sinus, the latter being covered in turn by the rubber cloth, $\mathrm{C}$, which fits closely about the metal shaft. "The spring at the top of the sliding rod, to which the agate pestle is attached at the bottom, can be adjusted to give any desired pressure or can be thrown back entirely to allow the pestle to be raised in removing the agate mortar. This is accomplished by loosening a set screw and dropping one of the four posts holding the mortar in place. The pestle should make about 200 revolutions per minute, the mortar moving very slowly in the same direction. The scraper keeps the ore in the center of the mortar and the combined rolling and sliding motion controlled by the ball and socket supporting side arm reduces the hardest ore very rapidly."-Iron Age.

revolution is too high. In our actual practice, three of these machines are operated, one or all, on a single table from the same shaft, the whole being covered with a glass case, divided by glass partitions, so as to exclude dust from the room and to separate the grinders. 


\section{ABRASION OF MORTAR AND PESTLE.}

The abrasion of crushing apparatus has been touched on in B.

The abrasion of pestle and mortar becomes far more manifest with machines like the above than with hand grinding, notwithstanding that each has its motion simulating as far as possible that of the hands. There is undoubtedly a very considerable introduction of silica into the sample from these sources, but in comparison with the amount invariably present in an igneous rock the quantity abraded is hardly of moment. Rocks normally free from silica, or nearly so, like many iron ores, are as a rule softer than igneous rocks and consequently cause less abrasion of the grinding implements.

Dr. E. T. Allen, in order to get an idea of the amount of silica introduced into an ordinary rock sample through the abrasion of the grinding implements, caused 200 grams of quartz sand to be ground in portions at a time till all passed a sieve of 150 meshes to the linear inch (60 to the centimeter), the time required being 19.5 hours. The mortar (635 grams) lost 0.189 gram in weight and the pestle (268 grams) lost 0.102 gram, a total of 0.291 gram, or 0.1455 per cent of the weight of the sand. The powder obtained in this case was less fine than that ordinarily sought at that time by rock analysts, but, on the other hand, the material was all quartz, a mineral much harder than most of those constituting the average rock.

W. Hempel ${ }^{a}$ has experimentally tested the effect of grinding in mortars of agate, glass, iron, and hardened steel; and has found that for hard bodies, like glass, hardened steel is far superior to agate, and that even mortars and pestles of green bottle glass showed much less abrasion than agate. In grinding 10 grams of glass to very fine powder the agate mortar and pestle, weighing 416 grams, lost 0.052 gram in weight (or $5 \mathrm{mg}$. per gram of glass), against a tenth of that loss with a hardened steel mortar and pestle of the same weight that had been some time in use.

Notwithstanding these observations in favor of steel, the use of metal in rock analysis is, as said above, forbidden by the conditions of the case. Still more so is that of glass because of its complex composition. If impurity can not be avoided, it is far better that it should be of one kind only, and that one productive of less disturbance than any other.

\section{E. WEIGHT OF SAMPLE.}

The sample when crushed or ground should weigh not less than 10 grams, and preferably 20 in case it should be necessary to repeat or advisable to employ unusually large portions for certain determinations, notably carbonic acid. Rock analysis has in this respect an 
advantage over mineral analysis, since material is almost always available in ample quantity and any desired number of separate portions may be used, whereas with a mineral the analyst is frequently compelled to determine many or all constituents in a single, often very small, portion of the powder. This course often involves delay and the employment of more complicated methods of separation than are usually necessary in rock analysis.

\section{WATER-GENERAL CONSIDERATIONS.}

\section{A. THE RÔLE OF HYDROGEN IN MINERALS.}

Although the condition of the hydrogen that is a constituent of all rocks is of less concern to the petrographer than that in minerals is to the mineralogist, yet the subject is of sufficient importance to the former and to the analyzer of rocks to warrant some discussion with regard to both rocks and minerals.

It is unnecessary to enter upon an historical discussion of the views that have prevailed as to the conditions of and the rôle that hydrogen plays in minerals and of the causes that have led to changes in those views. ${ }^{a}$ It will suffice to present the existing situation as it appears to the writer.

Broadly, we have to consider (1) hydrogen that is essential to the chemical or physical structure - that is, constitutional in either the chemical or the physical sense-and (2) that which is physically held otherwise and is unessential.

The subdivisions of the first of these greater divisions are $(a)$ hydrogen that is constitutional in the sense of possessing basic or acidic character, $(b)$ the hydrogen of water of crystallization, $(c)$ the hydrogen of solid solutions, which is commonly referred to the physical division.

For convenience in discussion we may adopt, as sanctioned by common usage, the term "water" instead of hydrogen, since in all its forms the hydrogen is expelled as water by heat.

\footnotetext{
$a$ The following authors in particular have contributed to our present understanding of the role of water in minerals:

Van Bemmelen, J. M., Zeitschr. anorg. Chemie, vol. 13, 1897, p. 233; and subsequent volumes down to the present time.

Tammann, G., Zeitschr. phys. Chemie, vol. 10, 1892, p. 255; vol. 27, 1898, p. 323; Zeitschr. anorg. Chemle, vol. 15,1897 , p. 319 .

Le Chatelier, H., Zeitschr. phys. Chemie, vol. 1, 1887, p. 396; Compt. Rend., vol. 104, 1887, pp. 1443, 1517; Bull. Soc. franc. min., vol. 10, 1887, p. 204.

Zambonini, F., Mem. Accad. Sci. fis. e mat. di Napoli, vol. 14, ser. 2, No. 1, 127 pp.

Tschermak, G., Zeitschr. phys. Chemie, vol. 53, 1905, p. 349; Zeitschr. anorg. Chemie, vol. 63, 1909, p. 169. Löwenstein, E., Zeitschr. anorg. Chemie, vol. 63, 1909, p. 69.

The list does not include all worthy of mention. Others had anticipated to some extent the major conclusions of Van Bemmelen and Tammann, without receiving the recognition that is now seen to be their due, as is fully set forth in the paper of Zambonini.
} 
The minerals of groups $a$ and $b$ are generally in a state of stable equilibrium with their surroundings over a considerable range of temperature and water-vapor pressure. Expulsion of their water is accomplished in theory either all at a certain temperature or vapor pressure or by steps. In those of group $c$ this is not true, but their water is progressively removable by increase of temperature or reduction of the water-vapor pressure of the surrounding medium. It is in respect to this third group that the above broader division shows deviation from the common, but the inclusion of this group in division 1 seems fully justified by the fact that in all three groups a change in at least some of the physical properties of the mineral necessarily accompanies loss of moisture. In other words, on the presence of the water in minerals of these groups depend certain of the physical properties. Without it the minerals as we know them could not exist. This dependence is noted, as a rule, most conveniently and quantitatively, by following the changes in certain optical properties that accompany variations in the water content, though at times other changes are more prominent. The hydrogen of subdivision $a$, probably also of $b$, forms part of the chemical molecule, while that in $c$ is supposed not to.

The subdivisions of the second of the larger divisions, aside from actual inclusions of liquid water, are less easy to define, as is apparent from the terms that are in more or less common use-hygroscopic, adsorbed, absorbed, occluded, imbibed, in addition to dissolved and solid solution already mentioned as transferred to the first division. Much uncertainty and confusion prevail, not only in the use of these terms but also in the meanings attached to them by different writers. Their number should certainly be reduced, for some are synonymous. Such differences as there may be between those physical conditions that seem to merit distinct recognition are probably rather of degree than of kind, conditioned by a more or less open structure of the solid particles. There is no certainty that a loose molecular or submicroscopic structure differs fundamentally in its attractive power for water from one in which the pores are visible, or in which, finally, only plane surfaces are concerned.

Certainly there does not seem to be any good ground for specific discrimination between hygroscopic water and that which is held to be adsorbed. Both are assumed to be results of surface attraction. Further, fine grinding of anhydrous minerals results in the assumption not only of loosely held (hygroscopic) water, removable at and below $100^{\circ}$, but also of water that may require a temperature of several hundred degrees for its complete expulsion. If the curve representing the progressive loss of such water at or slightly above the boiling point were found to end there, a difference of condition would have to be admitted. An actual interruption in the escape of 
water at this point seldom and perhaps never occurs. A break there may be, but not a total cessation of the upward trend of the curve representing loss of water. Such a break might be due merely to the fact that water happens to undergo a physical change at that point, and its sharpness will vary with different substances. Experiments made for the express purpose of testing this hypothesis are lacking, but are needed.

That, however, a distinction between this kind of water and that regarded as absorbed or imbibed may be justified is shown by the observation that the amount of the former depends on the fineness of division of the mineral particles, while there are many minerals, of which the zeolites afford excellent examples, besides some igneous glasses, which contain in the uncrushed state amounts of water that are very large, in well-defined minerals sometimes exceeding 20 per cent of their weight. This water, like that adsorbed, is progressively removable as the temperature is increased, and it too, in many cases, can be reabsorbed wholly or in large part, just as happens with the adsorbed water. But no change in the properties of the minerals accompanies this loss in either case, a fact which serves to differentiate them from those with water in solid solution. It is desirable that experiments should be instituted to ascertain what effect fine grinding would have on the water content of such minerals. So far as known to the writer, few determinations of the water in uncrushed minerals of this kind have been made for comparison with that in the same material ground. The meager data furnished by F. von Kobell ${ }^{a}$ afford little light on this point. ${ }^{b}$

Whatever may be the ultimate decision as to distinguishing between so-called adsorbed (hygroscopic) water and absorbed water, there is no question as to the difference in meaning of the words themselves, and they should not be used synonymously, as is frequently done. Adsorption implies surface condensation as contrasted with the idea of penetration conveyed by absorption, even though, as already hinted, there may be no fundamental difference in the causes that produce the states. It were better to avoid the use of the term absorbed in the above limited sense and to adopt instead imbibed. Absorbed might well be employed in a generic sense to cover the states of imbibition and adsorption.

a Jour. prakt. Chemie, vol. 107, 1869, p. 150; Chem. News, vol. 20, 1869, p. 119.

$b$ Since the above was written a paper by St. J. Thugutt has appeared (Centralbl. 1 . Min., Geol. u. Pal., 1909, p. 677) on "Sources of error in the determination of water in zeolites," which supplies in part the missing information. Thugutt shows, by a series of tests, that most zeolites and one or two other hydrous minerals examined take up water during grinding, but that the mineral apophyllite shows the opposite behavior. He further contends that the laws of Proust and Dalton maintain their full validity with respect to the zeolites, provided the material analyzed is strictly homogeneous and not too finely ground. As a corollary to the above he, too, advocates the double determination of water in mineral samples when fine grinding is necessary for the general analysis, and states that percentages should be reported on the basis of the water content of coarse material. 
As already said, the hydrogen in all conditions, essential as well as unessential, is expelled from inorganic minerals in the form of water by elevation of temperature. The temperature need not be high for water of crystallization and that physically held, but may be and in general is high for that which is basic or acidic. Two or more of these conditions can exist simultaneously in a mineral, and the temperatures at which they are in equilibrium with their surroundings may. overlap. As full criteria are lacking for the recognition of these conditions, the establishment of a probable formula is very often most difficult and not seldom impossible.

For convenience it may be well to follow established usage and call hygroscopic water that which is plainly not water of crystallization or of closer chemical union or of solid solution and is removable at or slightly above the boiling point of water or over strong dehydrants, always provided it is shown that the loss does not continue, even at a slower rate, above the given temperature. If such additional loss is shown to an appreciable amount, only careful study of the further behavior on heating and on subsequent exposure to a moist atmosphere will throw light on the nature of the water evolved. Water which is removable at the boiling point of water should also be removable by dehydrants like concentrated sulphuric acid or phosphorus pentoxide, provided time enough is allowed; the time will be shorter if a vacuum instead of atmospheric pressure is employed for the test. If a mineral undergoing progressive loss of water, by the aid either of a dehydrant or of heat below or above the boiling point of water, shows no change in its optical properties, it may be concluded that its hydrogen is not chemically held. The converse, that an optical change is proof of the presence of hydrogen in chemical combination is not, however, necessarily true, for it is not impossible that optical changes may occur from loss of imbibed water, the water of absorption of Zambonini. For minerals showing such apparent anomalies, like the zeolites, a micellar or alveolar or mesh structure is predicated by some writers. The high temperature is supposed to act on the walls of the mesh in a manner to weaken and finally overcome entirely their absorptive power. A contraction in volume accompanies the change; hence such minerals, when heated above a certain temperature, are able to reabsorb only a part or none of their original water content. The voids diminish in number and afterward refill with increasing slowness according to the degree of heat and its duration. Such physical change in structure is believed by Zambonini and others to be perhaps sufficient to account for observed changes in optical behavior.

Zambonini noticed, in experiments on the rehydration of partly dehydrated heulandite and other zeolites, sharp variations in the rate of absorption, not depending solely on the quantity of water 
evolved. Writing of heulandite, ${ }^{a}$ he regards this observation as "in complete disagreement with the hypothesis that the zeolites are solid solutions, since in this case there ought to have been continuous modifications in the rate of absorption, much less sharp than those observed with heulandite. $* * *$ What has been observed with this mineral finds perfect analogy in many properties of the hydrogels." He admits that there are close resemblances between the water of solid solutions and that of absorption [imbibition] compounds, and that the zeolites, "so far as concerns many of their physical properties, may be regarded either as solid solutions or as compounds of absorption." Whether or not Zambonini is right in his contention must be held an open question till further data on this obscure subject are procured and it has received adequate discussion.

The water of solid solutions, as has been said, is in full equilibrium with its surroundings for a given temperature and hygrometric state of the atmosphere. Therefore, no matter how fine a mineral containing it may be ground, the mineral should contain just as much, no more and no less, after grinding as before. This should, in theory, afford a means of distinguishing between such water and water that is simply absorbed or imbibed, if a distinction is really permissible, for it would seem as if imbibed water should be affected by the degree of division of the material containing it. The determination of this is, however, complicated by the fact that taking up of water usually accompanies fine grinding, so that if there were loss of water of imbibition on grinding this loss might be compensated more or less by adsorption or surface condensation. Viewed from this point, a reasonable doubt might arise whether imbibed water is really in a different condition from that called adsorbed.

In regard to water of solid solution it may not be amiss to refer to an experiment of Carl Barus, ${ }^{b}$ which has been cited more than once in support of the existence of solids containing water in solution and as explaining the high content in water of many igneous glasses, like the pitchstones and some obsidians, formed at great terrestrial depths. Barus heated to a high temperature in a closed bomb water and a powdered glass. After cooling under pressure, the glass was found to have taken up a very large volume of water (about equal to its own volume), and there remained a solid mass, still a glass, which seemed to be homogeneous and permanent. Time showed, however, that the water did escape, the glass becoming opaque and finally crumbling. It is also a fact, never published, that Barus was unable to cause all glasses to take up water in a similar way, and that it was only with the common soft glasses that the experiment succeeded as above outlined. This illustration serves to emphasize the conclu- 
sion that the igneous glasses above mentioned may be simply supercooled liquids of extreme viscosity and not comparable with the solutions represented by many crystallized bodies.

As to the chemically combined hydrogen, this should be evolved for each degree of hydration or each manner of linking in the molecule at a definite temperature with a constant vapor tension so long as any of the material in the state undergoing change is still present. This is in the main so for the true hydrates, but to a much less degree for those minerals which hold their hydrogen with a firmer bond. The latter usually require a much higher temperature for dehydration than the hydrates, and to account for the frequently observed continuous instead of discontinuous escape of their water, there has been suggested a contraction or sintering, whereby greater resistance to escape is offered, and also a possible difference of temperature between the interior of the mass of particles and those nearer the external source of heat (Zambonini).

In explanation of the fact that the last traces of water are often removable from undoubted hydrates at a far higher temperature than is required for the major part, I would offer the suggestion that the water of crystallization may have been displaced as such at the proper temperature and that any water still remaining above that temperature is some of the original water of crystallization now held physically by surface attraction.

The interpretation of the analytical results obtained for hydrogen is thus, to repeat briefly, often a most difficult problem, one requiring much knowledge of mineralogy and of the relationships between different minerals and groups of minerals. In many cases it is quite impossible, with our present knowledge, to discriminate practically between the hydrogen of water of crystallization and that of closer chemical union, and even between these and some of that physically held, especially when more than one of these conditions exist together. Ordinarily the two former show more or less simple stoichiometrical relations to the other constituents, but the hydrogen of chemical constitution regarded as basic or acidic offers frequent exceptions. This may be and often is due, no doubt, to its forming one of two or more members which are simultaneously present in isomorphous relationship tọ each other, of which hydroxyl and fluorine show striking examples in a number of minerals.

For the methods that have been applied in the study of water in minerals and the hydrogels reference must be made in part to the above-cited papers of Zambonini, Van Bemmelen, Tammann, and Löwenstein, and also of A. Hamberg, ${ }^{a}$ since they apply more to the examination of minerals than of rocks. To these may be added 
papers by W. W. Coblentz, ${ }^{a}$ who has applied an entirely noviel method to discriminate between molecular water and that in the form of hydroxyl. As these papers have escaped the attention of mineralogists, it may be well to quote from the author's summary, although his deductions may not prove to be warranted:

The transmission and reflection spectra of at least 120 compounds have been examined, many of them to $15 \mu$., by means of a mirror spectrometer, a rock-salt prism, and a Nichols radiometer. The interpretation of the results is based on the assumption that if the union of the oxygen and the hydrogen in the molecule is similar to that in water, then the absorption spectra of minerals containing these two elements thus united should show the absorption bands of water superposed upon the absorption spectrum of the other constituents. On the other hand, minerals containing oxygen and hydrogen as water of constitution should not show the water bands, except hydroxyl groups, which should show a band at $3 \mu$.

The results show that of about 30 minerals containing "water of crystallization" there are no important exceptions to the rule that they should show the bands of water. On the other hand, the one important exception to the rule that minerals containing "water of constitution" should not show water bands is cane sugar. Minerals containing hydroxyl groups generally have a marked band at $3 \mu$. Sulphates have a strong band at $4.55 \mu$ and a less constantly recurring band at $9.1 \mu$, due to the $\mathrm{SO}_{4}$ ion. On the other hand, silicates do not have such definite bands, which would seem to indicate that the structure of the silicate radical is different in each mineral containing that element.

The following tentative scheme of classification summarizes the foregoing very brief discussion. In this discussion I have refrained from any direct comparison of the condition of the water held by amorphous bodies with that in crystallized minerals. Our knowledge of colloids and gels is too meager to make comparison profitable. Where, or whether at all, such bodies fit in the classification is therefore left open.

\section{Provisional classification of the condition of hydrogen in minerals.}

A. Essential or structural hydrogen:

a. Basic and acidic. Discontinuously removable for Loss of any of these necesb. In water of crys- $\left\{\begin{array}{l}\text { a given form of union-that } \\ \text { is, at a fixed temperature or }\end{array}\right\} \begin{aligned} & \text { sarily involves a change } \\ & \text { in physical properties of }\end{aligned}$ tallization. vapor pressure. c. In solid solution (dissolved)................ the mineral, for example,

B. Unessential hydrogen:

a. Liquid inclusions of water.

b. Absorbed as water:
$\alpha$. Adsorbed, including as "hygroscopic" that
removable at the boiling point of water.
$\beta$. Imbibed, as water is held by a sponge......
Progressively removable with increase of tempera- ture or reduction of vapor pressure without necessa. rily involving changes in optical properties.

a Carnegie Institution of Washington, Pub. No, 65, 1906; Bull, Bureau of Standards, vol. 2, No. 3, 1906, p. 457 , 


\section{B. WATER TAKEN UP OR LOST ON GRINDING.}

Before considering methods of determination attention must again be called to the effect of fine grinding of mineral powders on their ability to take up from the air and retain at temperatures far above $100^{\circ}$ very appreciable amounts of water, even in excess of 1 per cent. (See p. 53.) The following table contains typical data on this subject. For fuller details see the paper already cited. ${ }^{a}$

Water content of powders of varying fineness, ground in air.

\begin{tabular}{|c|c|c|c|c|}
\hline \multirow{2}{*}{ No. } & \multirow{2}{*}{ Material. } & \multirow{2}{*}{ Condition. } & \multicolumn{2}{|c|}{ Water. } \\
\hline & & & $\begin{array}{l}\text { Below } \\
100^{\circ} \text {. }\end{array}$ & $\begin{array}{c}\text { Above } \\
100^{\circ} .\end{array}$ \\
\hline $\begin{array}{l}1 \mathrm{a} \\
1 \mathrm{~b} \\
1 \mathrm{c} \\
2 \mathrm{a} \\
2 \mathrm{~b} \\
3 \mathrm{a} \\
3 \mathrm{~b} \\
4 \mathrm{a} \\
4 \mathrm{~b} \\
5 \mathrm{a} \\
5 \mathrm{~b} \\
6 \mathrm{a} \\
6 \mathrm{~b}\end{array}$ & 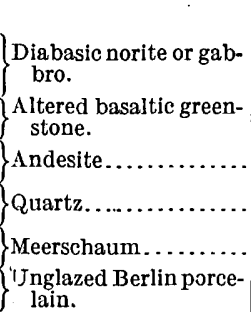 & 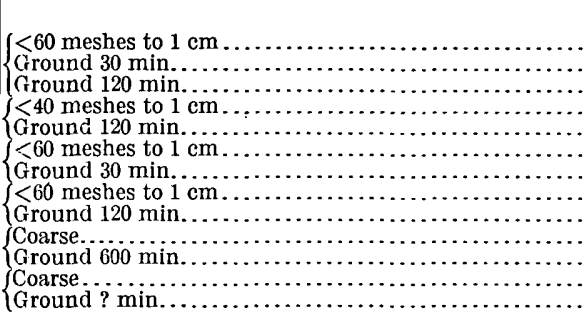 & $\begin{array}{r}\text { Per cent. } \\
0.03 \\
.10 \\
.74 \\
.22 \\
1.70 \\
1.01 \\
1.18 \\
\text { None. } \\
.35 \\
5.76 \\
6.33 \\
\text { None. } \\
.62\end{array}$ & $\begin{array}{r}\text { Per cent. } \\
0.66 \\
.66 \\
1.00 \\
3.13 \\
4.19 \\
3.40 \\
3.54 \\
.06 \\
.45 \\
6.79 \\
9.81 \\
\text { None. } \\
.18\end{array}$ \\
\hline
\end{tabular}

On the other hand, water of crystallization may be expelled from minerals by long-continued grinding. Thus the content in water of a specimen of gypsum was reduced from over 20 to about 5 per cent. ${ }^{b}$ The inference is obvious that water of crystallization should be determined on unground material, if possible.

The following excerpt from the work of Doctors Day and Allen ${ }^{c}$ on the feldspars, already cited, has direct relation to the above:

There is another error to which accurate specific-gravity determinations upon powdered minerals will be subject unless suitable precaution is taken. The exposure to the air during the period of grinding the samples gives opportunity for the condensation of sufficient atmospheric moisture upon the grains to affect the weight in air. The amount varies measurably with the size of the grains, as will be seen from the accompanying data, and probably with the degree of saturation of the atmosphere and the time of exposure.

a Jour. Am. Chem. Soc., vol. 30, 1908, p. 1120; Chem. News, vol. 98, 1908, pp. 205, 215.

$b$ Idem.

c Pub. Carnegie Institution of Washington, No. 31, pp. 56-57; Am. Jour. Sci., 4th ser., vol. 19, 1905, p. 93. Consult also Mauzelius, R., Sveriges Geol. Undersökning, Arsbok 1, 1907, No. 3; and Hillebrand, W. F., Jour. Am. Chem. Soc., vol. 30, 1908, p. 1120; Chem. News, vol. 98, 1908, pp. 205, 215; and the footnote on $\mathrm{p} .44$ of the present volume. 
Determination of moisture in 1 gram of powdered mineral upon exposure to the air.

$[<=$ finer than. $>=$ coarser than.]

\begin{tabular}{|c|c|c|}
\hline Mineral. & $\begin{array}{c}\text { Fineness (meshes to } \\
\text { the linear inch). }\end{array}$ & Moisture. \\
\hline 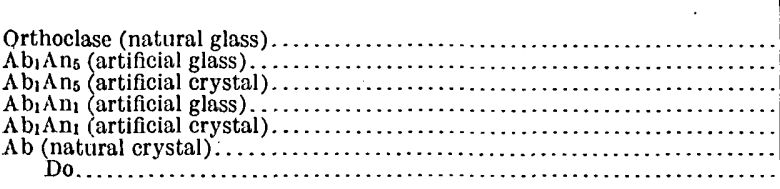 & 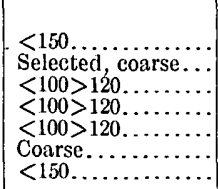 & $\begin{array}{r}G r a m . \\
0.0061 \\
.0000 \\
.0010 \\
.0007 \\
.0010 \\
.0006 \\
.0069\end{array}$ \\
\hline $\begin{array}{l}\text { Orthoclase (natural crystal) } \ldots \ldots \ldots \ldots \\
\text { Orthoclase (same sample) } \ldots \ldots \ldots \\
\text { Do... }\end{array}$ & $\begin{array}{l}<120>150 \ldots \ldots \ldots \\
<1.50 \ldots \ldots \ldots \ldots \\
\text { Still finer............ }\end{array}$ & $\begin{array}{l}.0011 \\
.0031 \\
.0059\end{array}$ \\
\hline $\begin{array}{l}\text { Orthoclase (artificial glass) } \ldots \ldots \ldots \ldots \ldots \ldots \ldots \ldots \ldots \ldots \ldots \ldots \ldots \ldots \ldots \ldots \ldots \\
\text { Orthoclase (portion of same) } \ldots \ldots \ldots \ldots \ldots \ldots \ldots \ldots\end{array}$ & $\begin{array}{l}\text { Everything }<100 \ldots \\
>150 \ldots \ldots \ldots \ldots \ldots\end{array}$ & $\begin{array}{l}.0065 \\
.0022\end{array}$ \\
\hline
\end{tabular}

In the last two groups note that the moisture in graded portions of the same sample varies with the fineness.

We also verified the conclusion of Bunsen $a$ that this absorbed moisture is not altogether removed at temperatures only slightly above $100^{\circ}$, but requires $600^{\circ}$ to $800^{\circ}-$ equivalent to low red heat. Several samples for which the moisture had been determined were laid away in corked test tubes for a number of weeks, after which redetermination gave exactly the former value.

It is worth noting in this connection that these measured quantities of absorbed water are of the same order of magnitude as those usually obtained for the water content in feldspar analyses, where again, of course, the finer the sample is ground for the analysis the greater the possible error from this cause. It may be that a part and occasionally all of the moisture usually found in these analyses is adsorbed and the significance of its presence there mistaken.

\section{IMPORTANCE OF EMPLOYING AIR-DRY POWDER FOR ANALYSIS.}

The time-honored custom of drying a powdered specimen before bottling and weighing has long seemed to me one that has no sound basis in reason. Its object is plain, namely, that of securing a uniform hygroscopic condition as a basis for convenient comparison of analytical results, because some rock powders contain more hygroscopic moisture than others and the amount is dependent further on the degree of fineness of the powder. Nothing, however, is more certain than that by the time the dried substance is weighed it has reabsorbed a certain amount of moisture, small indeed in most cases, but very appreciable in others; and, further, that with every opening of the tube moisture-ladened air enters and is inclosed with the remainder of the dry powder. Therefore, it may very well happen that a powder at first dry will, after several openings of the tube, especially at considerable intervals, be nearly as moist as when first inclosed. Dittrich has expressed views similar to the above. 
It is preferable to weigh the air-dry powder and to make a special determination of moisture. If all the portions necessary for an analysis are weighed out one after another, or even at different times on the same day, the error due to difference of hygroscopicity in dry and moist weather, which for most of the separate portions is an entirely negligible quantity, is eliminated. Only in the main portion, in which silica and the majority of the bases are to be estimated, can it ever be an appreciable factor. Especially is this true if a rather coarse powder is employed, such, for instance, as passes a sieve of 30 to 60 meshes to the linear centimeter. In powders of this grade the moisture will fall very often below 0.1 per cent, whereas in those that have been ground for one to two hours it may easily exceed 1 per cent.

\section{ARGUMENT IN FAVOR OF INCLUDING HYGROSCOPIC WATER IN THE} SUMMATION.

The question has been asked: "If the so-called hygroscopic water is not always such, but not infrequently includes essential water, why are its separate determination and entry in the analysis entirely unnecessary? Why make a distinction which after all may not be a true one?" The question involves the further consideration of the need in any event of including the loss at $100^{\circ}$ or $110^{\circ}$, a question that has been covered to some extent in section $\mathrm{A}$.

It would seem to be a reasonable desire on the part of petrographers to have all analyses referred to a moisture-free basis in order that they shall be strictly comparable. This involves the omission of the "hygroscopic" water from the list of constituents, which would be eminently proper were it always possible to be sure that the loss at $100^{\circ}$ to $110^{\circ}$ truly represents such water. Since it very often represents more, and the determination as to whether or not it does in each case is not always possible and would add to the time required for the analysis, it seems necessary to include this water. What errors may arise from its exclusion the following rather extreme case well illustrates: Certain rocks of Wyoming in powder form lost from 1 to 2 per cent of moisture at $110^{\circ}$. That not even an appreciable fraction of this was truly hygroscopic is fully demonstrated by the fact that the uncrushed rocks lost the same amount; yet the rule followed by many chemists and petrographers would have involved the removal of all this water as a preliminary to beginning the analysis, and not only would a most important characteristic have passed unnoticed, but the analyst would have reported an incorrect analysis, intiting to false conclusions and possibly serious confusion. (See also second paragraph of footnote, p. 44, and examples cited on p. 69.)

Hence it may be said that the estimation of the loss at $100^{\circ}$ or $110^{\circ}$ and its separate entry in the analysis are advisable as affording to the 
lithologist an indication of the mineral character of one or more of the rock constituents, thus perhaps confirming the microscopical evidence or suggesting further examination in that line. A marked loss in weight at $100^{\circ}$ by a coarse powder other than that of certain igneous glasses may be taken as almost positive proof of the presence of zeolites or other minerals which characteristically contain loosely held water. It has been objected that the hygroscopic moisture varies with the degree of comminution of the sample and with the condition of the air at the time of weighing, and that it is therefore improper to incorporate it in the analysis; but this variation is ordinarily not at all great if the powder is coarse.

\section{WATER.-METHODS OF DETERMINATION.}

With the coarse powders that are needed to afford a true value for the hydrogen content of rocks and minerals, one or another of the methods described in this chapter will be found more or less unsatisfactory, especially with those materials that lose their hydrogen at very high temperatures or that are difficult to decompose with fluxes. Unfortunately, at present no directions can be given to cover all such cases. In any event duplicate determinations should be made until the analyst has acquired the experience that will permit him sometimes to make exceptions.

\section{A. INDIRECT METHODS.}

a. GENERAL CONSIDERATIONS.

The means employed for the indirect determination of water differ according as fractional or total amounts are needed. For the latter, simple ignition is adopted; for the former, exposure to the effects of dehydrating reagents or different temperatures. These methods, as ordinarily carried out, while exceedingly simple, are affected in most cases by serious errors, which will be considered in turn in their proper places. The methods should not be employed, therefore, when accuracy is essential, unless it is known that the errors are too slight to be significant or unless modifications are introduced to guard against them. A complication, sometimes serious, is added by the fact that with coarse powders the time required for expulsion of the water may be much greater than when a fine powder is used.

\section{b. METHODS INVOLVING THE USE OF DEHYDRATING AGENTS.}

Calcium chloride, sulphuric acid, and phosphorus pentoxide are the chemical dehydrants commonly employed in mineral analysis. Of these sulphuric acid finds most extended use, generally in a condition of high concentration, but in varying degrees of known con- 
centration if detailed study of the behavior of a mineral during dehydration is in progress. I have shown ${ }^{a}$ the importance of employing in desiccators only sulphuric acid of maximum dehydrating power when the full effect of the acid is desired. That which has stood long in desiccators that are in constant use is by no means so effective as fresh strong acid. Furthermore, the acid becomes dark from access of organic matter from dust or from the lubricant of the vessel, and this gives rise to the formation of appreciable amounts of sulphur dioxide.

A mineral which loses much water over a strong dehydrant may need an exposure of several days or even weeks for its complete extraction. If the weighings are made from day to day, the apparent limit may be reached long before all water really removable has been taken up by the acid. Whenever the crucible, after weighing, is replaced in the desiccator it is no longer in a dry but a more or less moist atmosphere, and its contents, even when covered, sometimes absorb a part of this moisture and retain it so persistently that the acid is unable to bring the powder beyond its previous state of dryness in the next twenty-four hours. In fact, it may be unable even to reach it unless greater time is allowed. An experiment on tyrolite, made and published many years ago, illustrates this point in part:

Experiment of drying 1 gram of tyrolite over strong sulphurio acid.

\begin{tabular}{|c|c|c|c|}
\hline $\begin{array}{c}\text { Time } \\
\text { exposed. }\end{array}$ & Loss. & $\begin{array}{c}\text { Time } \\
\text { exposed. }\end{array}$ & Loss. \\
\hline \multirow{2}{*}{$\begin{array}{r}\text { Hours. } \\
18 \\
26 \\
23 \\
24 \\
23 \\
24 \\
25\end{array}$} & \multirow{2}{*}{$\begin{array}{c}\text { Gram. } \\
0.0231 \\
.0083 \\
.0029 \\
.0012 \\
.0008 \\
.0001 \\
.0003\end{array}$} & $\begin{array}{r}\text { Hours. } \\
24 \\
24 \\
48 \\
24\end{array}$ & $\begin{array}{r}\text { Gram. } \\
0.0002 \\
.0003 \\
.0006 \\
.0002\end{array}$ \\
\hline & & 283 & .0380 \\
\hline
\end{tabular}

The experiment might reasonably have been considered ended after the one hundred and thirty-eighth hour, when a loss of but 0.1 milligram was shown during twenty-four hours; but nevertheless a nearly steady loss of $0.3 \mathrm{mg}$. per day took place for six days more, and might have been longer observed but for the interruption of the experiment. The sample was in fine powder. Had it been coarse the rate of dehydration would have been much slower. The time can, of course, be greatly reduced by operating in a vacuum. Given sufficient time, the use of a dehydrant entirely without water-vapor tension should be as effective as a temperature corresponding to the boiling point of water; provided the surrounding medium is itself entirely dry. 
The indirect method can be made more accurate by heating the mineral or rock in a weighed tube, through which a current of air or other gas can be passed, either dry or of definite moisture content, according to the object in view, and then determining the loss in weight of the tube and its contents. If the drying agent for the gas is phosphorus pentoxide, it is well to cause the gas to pass first over or through calcium chloride or sulphuric acid, for the effectiveness of the pentoxide rapidly lessens as it becomes glazed with metaphosphoric acid.

c. METHODS INVOLVING THE APPLICATION OF HEAT.

$\alpha$. Fractional determinations.-As to the temperature to be adopted for drying in order to determine so-called hygroscopic moisture, the practice has varied at different times and with different workers, ranging from $100^{\circ}$ to $110^{\circ}$. For the great majority of rock specimens it is quite immaterial which of these temperatures is adopted, since no greater loss is experienced at the higher than at the lower temperature, given a sufficient time for the latter. It is the present practice in this laboratory to employ a toluene bath giving a temperature of about $105^{\circ}$. Should the results show a very unusually high loss, the powder is reheated at, say, $125^{\circ}$, in order to learn if the loss is progressive with increased temperature, as this knowledge is important for the interpretation, of results. 'To insure accuracy this experiment should never be made in crucibles or dishes which must be cooled in a desiccator. One instance will suffice: A gram of a mineral mixture containing about 17 per cent of water, of which about 3 per cent was driven off at $100^{\circ}$ and 8 or 9 per cent at $280^{\circ}$, was, after several hours heating at the latter temperature, placed in a desiccator over sulphuric acid and weighed as soon as cold, then replaced and again weighed the next day. It had regained $1 \frac{1}{2}$ per cent of its original weight, although the desiccator was tightly closed and the crucible covered, showing apparently a drying power superior to that of the acid, or having, in other words, a vapor tension less than that of the acid.

A specimen of tyrolite was found on one occasion to lose 10.34 per cent at $280^{\circ}$, and on another occasion 14.33 per cent. In the latter case the drying and heating at progressive temperatures had continued during a period of 528 hours, the weighings being made usually from day to day; whereas in the former the duration of the experiment was much shorter and the.. intervals between weighings were but a few hours each.

An important research of G. Friedel ${ }^{a}$ well shows what errors are possible in the determination of this easily removable water, since he found that certain zeolites which had been largely dehydrated but not

a Bill. Soc. min., vol. 19, 1896, pp. 14, 94; Compt. Rend., vol. 122, 1896, p. 1006. 
heated to the point of rupture of the molecular net could then absorb, instead of water, various dry gases in which they might be placed, as carbon dioxide, ammonia, carbon disulphide, and others, even air in large quantities, and certain liquids. In the light of this observation the cause of the great increase of $1 \frac{1}{2}$ per cent in weight of the partially dehydrated mineral mentioned above may very possibly be attributed to air from the desiccator instead of moisture, as was at the time supposed. At any rate, as Friedel says, the danger of accepting a loss in weight as an index of the amount of water lost is clearly shown, and thus that method of determining water is for many cases fully discredited. Just what method to adopt must be largely left to the judgment of the operator, who will often be guided by the mineral composition of the rock as revealed by the unaided eye or the microscope.

Friedel ${ }^{a}$ indicates a means for determining the true weight of water lost by minerals behaving like the zeolites, even without collecting the water lost, namely, by driving out of the dehydrated and weighed mineral, under proper precautions, any air it may have absorbed in the process of drying and cooling, and collecting and measuring this air and thus finding its weight, which, added to the apparent loss, gives the true contents in water.

But aside from the above reasons for avoiding the use of indirect methods of water determination, there is another very important one. They never give correct results where air baths are used in the ordinary way, for the reason that the drying is performed in an atmosphere far from dry, and the substance can not, therefore, lose all its moisture. As examples, if the indirect method shows 0.12 and 0.75 per cent in two samples, the direct method may afford 0.17 and 1.00 per cent, respectively. Similar observations have been repeated many times, and admit of no dispute. They apply as well to rocks as to minerals. Therefore, if the moisture content is more than an insignificant factor the indirect method must be avoided, or so arranged as to insure a constantly changing dry atmosphere.

To effect this the powder should be heated in a weighed tube, through which a current of dry air can be passed, and allowed to cool therein, after which a second weighing gives the loss of moisture. The degree of drying will depend on the nature of the reagent used for drying the air, sulphuric acid being more effective than calcium chloride, and phosphoric pentoxide most effective of all. When using the last, it is well to cause the air first to pass over or through one of the first-named dehydrants, for its effectiveness rapidly lessens as it becomes glazed with metaphosphoric acid. For experiments carried out at room temperatures no bath is required, but for artificial temperatures the tube should pass through a suitable thermostat. 
$\beta$. "Loss on ignition" method for total water.-In a few cases the simple loss on ignition of a rock will give the total water with accuracy, but in the great majority there are so many possible sources of error that this old-time method can rarely be used with safety. Only when the rock is free from fluorine, chlorine, sulphur, carbon, carbon dioxide, and fixed oxidizable or reducible $\left(\mathrm{MnO}_{2}\right)$ constituents can the loss be accepted as the true index of the amount of water present, and it is rare that a rock is met with fulfilling these conditions, especially as to the absence of ferrous iron. Blast ignition in presence of carbon dioxide alone of the above list may give a correct result, after separate estimation of the carbon dioxide, provided this emanates from carbonates of the earths and not from those of iron or manganiese. Another source of error lies in the fact that from some minerals the hydrogen is not all driven out, even at the highest temperature obtainable by a blast lamp. Such heat may, on the other hand, drive out other difficultly volatile constituents, as the alkalies. This happens particularly. when mixtures of alkaliferous silicates and alkaline-earth carbonates are concerned, such as argillaceous limestones.

The long-maintained idea that in presence of ferrous iron a sufficiently correct result is obtainable by adding to the observed loss an amount needed for oxidizing all ferrous iron is not justifiable. There can be no certainty that the oxidation has been complete, especially. in the case of readily fusible rocks, and at the high temperature of the blast a partial reduction of higher oxides is not only possible but sometimes certain. ${ }^{a}$ The inability to insure complete oxidation by simple ignition is illustrated in the case of precipitated ferric hydroxide which has been ignited in contact with its filter paper. If the quantity was in any degree large it is sometimes decidedly magnetic, presumably from presence of magnetic oxide, which no amount of heating wholly oxidizes, especially in the larger grains. Neither is evaporation with nitric acid and reignition sufficient to destroy the magnetic property of the oxide, as has been claimed.

P. G. W. Bayly has published a paper ${ }^{b}$ on recent methods of rock analysis, in which he gives the following method for determining water. The total ignition loss is corrected for carbon dioxide when this is present, and for ferrous oxide by determining the ferrous iron both before and after ignition. Deduction of the separately determined moisture gives finally the more firmly held water. It need. hardly be said that this method presupposes very exact determinations of both moisture and ferrous oxide.

a H. Warth (Chem. News, vol. 84, 1901, p. 305, also mentions this and draws attention to the effect of alumina, not only in decolorizing ferric oxide when the two are heated together, but also in restraining the reduction of the latter to the magnetic oxide.

$b$ Australasian Assoc. Adv. Sci., Adelaide meeting, 1907. 


\section{B. DIRECT METHODS WITHOUT ABSORPTION TUBES-PENFIELD'S METHODS.}

a. FOR MINERALS EASILY DEPRIVED OF THEIR WATER.

If no other volatile constituents than water are present, the beautifully simple method first used by Prof. G. J. Brush and extended by Prof. S. L. Penfield ${ }^{a}$ leaves nothing to be desired for accuracy. It consists simply in heating the powder in a narrow tube of hard glass, enlarged at the closed end and provided with one or two further enlargements in the middle to hold the water and prevent its running back and cracking the hot glass. A capillary glass stopper fitted in with rubber tubing prevents loss of water by circulating air currents. The tube being held horizontally; the bulb is heated to any required degree by the Bunsen or blast flame. Moistened filter paper or cloth

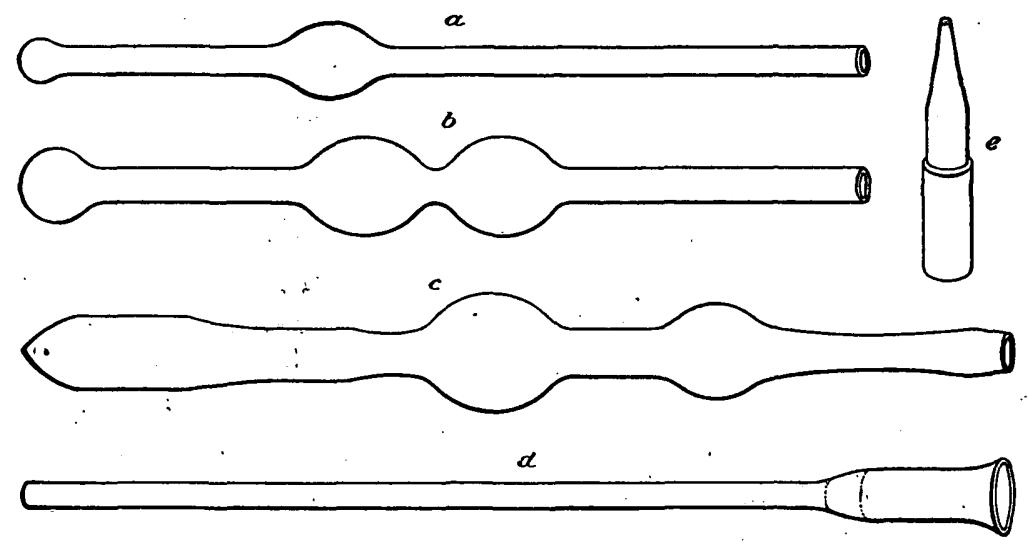

FIG. 11.- Penfield's tubes for water determination in minerals. . $a, b, c$, Different forms of tubes; $d$, thistle tube for introducing the powder; $e$, capillary-tipped stopper.

wound about the cooler parts of the tube insures condensation of all water. The heated end being finally pulled off, the tube is weighed after cooling and external cleansing, and again after the water has been removed by aspiration. For most rocks, as they contain little water, central enlargements of the tube are hardly needed.

Various forms of tubes used by Penfield are shown in fig. 11 .

Before using, even if apparently dry, "these tubes must be thoroughly dried inside, which is best accomplished by heating and aspirating a current of air through them by means of a glass tube reaching to the bottom."

How this simple tube is made to afford entirely satisfactory results with minerals, even when carbonates are present, is fully set forth in the paper cited. 
Comparatively fêt rocks are altogether free from other volatile constituents. Hence for refined work the application of this apparatus in the simple manner above set forth is limited. It may, however, be used with the addition of a retainer for fluorine, sulphur, etc., in the shape of calcium, lead, or bismuth oxides. It gives, of course, the total water.

b. FOR MINERALS NOT EASILY DEPRIVED OF THEIR WATER.

When minerals are present that do not give up their water wholly, even over the blast, as talc, topaz, chondrodite, staurolite, etc., Penfield's simple combination of fire-brick and charcoal oven, depicted in fig. 12, must be used, either with or without a retainer for fluorine, as circumstances demand. The part of the tube in the fire is to be protected by a cylinder of platinum foil tightly sprung about its end, and the part outside by asbestos board, as well as by wet cloth or paper. A piece of charcoal is likewise laid on the tube, as well as

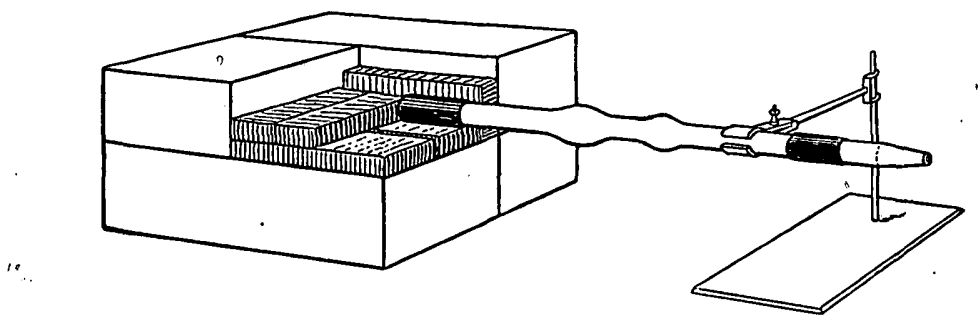

FIG. 12.-Penfield's fire-brick and charcoal oven for use in determining water.

beneath and behind, and the blast flame is given a horizontal direction, so as to play upon the side of the apparatus. In this way a most intense temperature can be reached.

In whichever way the apparatus may be used, the water found is the total water, from which that found separately at $105^{\circ}$ may be deducted if desired.

\section{DIRECT METHODS WITH ABSORPTION TUBES.}

a. GENERAI, CONSIDERATIONS.

In general it is preferable to collect and weigh the water in suitable absorption tubes after its expulsion from the mineral or rock. The method is especially suited for the determination of total water or of that escaping above $105^{\circ}$ when the apparatus of Gooch (e, p. 78.) is employed. It is less so for those fractional determinations where the escape of water is very slow at the different temperatures, for the absorption tube may in such cases gain weight, other than that of water from the mineral, sufficient to introduce appreciable error. This may sometimes be corrected to a certain extent by running $\Omega$ long blank test. 
A precaution too often overlooked is to see that the drying agent used for the air current is the same as that in the absorption tube in which the water from the sample is collected-not only the same in kind, but as near as may be in strength as well, especially with calcium chloride. Therefore, the contents of drying and absorption apparatus should be changed often, and when refilled the reagents should be taken from the same stock. Fresh calcium chloride in absorption tubes should be exposed to a current of carbon dioxide and the excess removed by air before use. Furthermore, all connections should be as nearly as possible glass against glass, with the least exposure of rubber. With long rubber connections the error may be a very sensible plus one. Again, between refillings of the absorption tube the direction of the air current through it should always be the same. Care must also be taken that the conditions at the several times of weighing of the tujes are nearly alike as to temperature and hygrometric state of the atmosphere, for the afternoon weight of a tube may be appreciably different from that on the following morning. It is well, therefore, to avoid error from this cause by approximately counterpoising the absorption tube by another of about the same volume and weight. Finally, the electrifying effect of wiping a tube before placing it on the balance must not be overlooked. In my experience this has often amounted to as much as 1 to 2 centigrams in a balance resting on glass (always an increase in weight) after wiping with a clean linen handkerchief. The charge can be removed by repeated application of the hand, but disappears slowly without such aid. A strongly electrified condition makesitself apparent by marked irregularities in the swing of the needle. While more pronounced in cold weather, the phenomenon is not peculiar to winter. ${ }^{a}$

The absorption tubes should not in general weigh over 30 grams each, filled with calcium chloride. Those preferred by me are shown in fig. 15 (p. 77), and are 10 to $11 \mathrm{~cm}$. over all in height. Sulphuric acid as an absorbent has no apparent advantage over calcium chloride if the drying apparatus for the air contains also calcium chloride of the same absorptive power as that in the collecting tube.

\section{b. PENFLELD'S PROCEDURE.}

The simplest of these methods as to apparatus, and one permitting, by the use of auxiliary arrangements such as are shown and described on page 77, the determination of the hygroscopic as well as any other fraction of the water, is the following glass-tube arrangement (fig. 13) of Penfield, ${ }^{b}$ whereby the brick and charcoal oven already referred to (fig. 12) comes again into play, but without the half brick shown in that figure. 
The tube is of about $15 \mathrm{~mm}$. internal diameter, and is fitted with two platinum cylinders at $A$, one inside, the other outside, where the heat exposure is to be most intense.

These are made from pieces of platinum foil, about $0.07 \mathrm{~mm}$. in thickness and 8 by 11 $\mathrm{cm}$. in diameter, which have been previously bent around glass tubes of such a size that when applied to the combustion tubing the spring of the metal will hold them in place. A large platinum boat, 7 to $8 \mathrm{~cm}$. long and 11 to $12 \mathrm{~mm}$. in diameter, with a cross section like $b$, should be used, since this will readily hold a gram of mineral mixed with 5 grams of sodium carbonate. * * * The tube is placed in the angle formed by the charcoal lining, some pieces of charcoal are placed at the sides in front, leaving an opening through which the flame may be directed, and an additional piece is laid on top. The tube can readily be brought to a full white heat, and by forcing a slow current of dry air through the apparatus the carbon dioxide resulting from the decomposition can be removed and the water carried over into the weighed absorption tube. The glass fuses between the platinum casings, and in a number of experiments that have been tried there has not been a single instance where the glass tube has broken or shown any indication of breaking. After heating, the tube will not crack if.it is left to cool slowly on the charcoal, but it can not be used a second time. $* * *$ At the high temperature to which the glass is subjected it of course becomes very soft and the ends must be properly supported; also the rubber connections and absorption

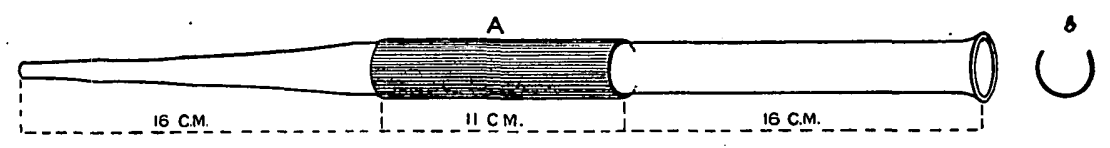

FIG. 13.-Tube for water determination according to Penfield. A, Outer protecting covering of platinum foil. A second similar foil on the inside prevents the glass from collapsing when heated to softness. $b$, Cross section of platinum boat.

apparatus must be carefully screened by asbestos board. By constructing a cover for the boat no material need be lost by spattering, and after making the water determination the contents may be used for the remainder of the analysis.

The inner cylinder of platinum serves to prevent the glass from collapsing as it softens, whereby distortion of the boat would result and its withdrawal for further examination of its contents would be impossible.

C. STEIGER'S APPIICATION OF THE TOLUENE OVEN.

The apparatus devised by George Steiger consists simply of a $\mathrm{U}$ tube of home construction and a small toluene oven, in the cover of which is an opening of suitable shape to receive the tube (fig. 14). The open space between the uprights is closed when in operation by a piece of asbestos board. The horizontal end of the tube is made long, so that in the event of much water being given off it can be readily driven over into the absorption tube by a low flame, which if applied from the first may prevent any appreciable condensation of water before reaching the calcium chloride. The interior of the tube having been first freed from adhering moisture by a current of dry air and the absorption tube attached, the stopper is lifted without 
shutting off the air current, and the weighed powder quickly introduced by means of the clean metal trough in which it has been weighed (fig. 4, p. 33). The stopper is as quickly replaced and the

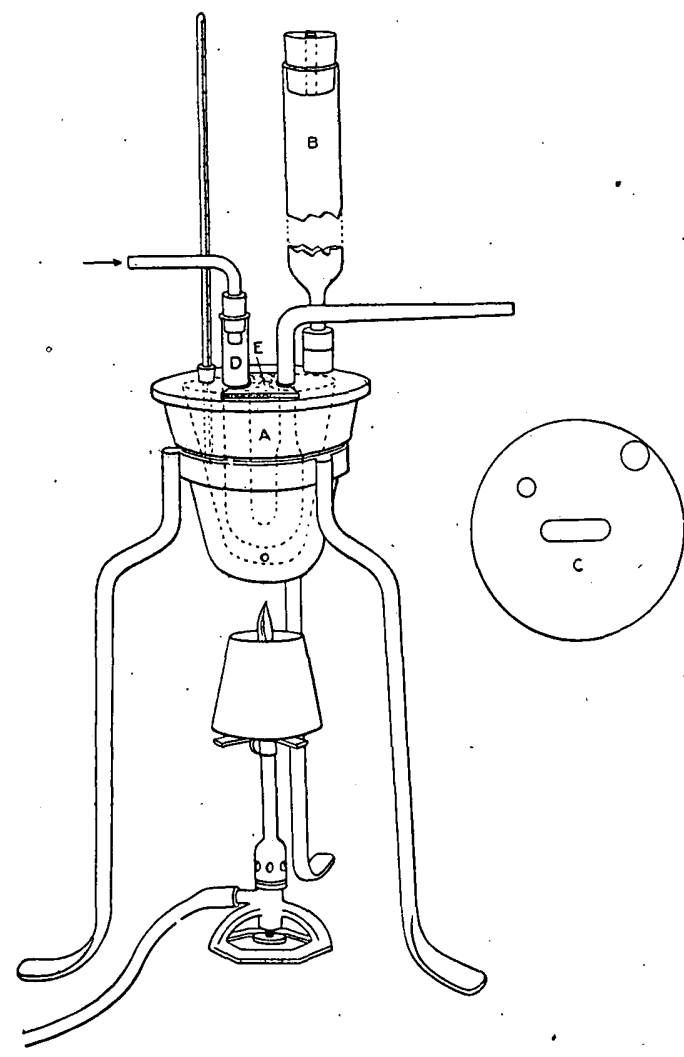

FIG. 14.-Steiger's form of drying apparatus. A, Toluene oven; $\mathrm{B}$, condenser, about $50 \mathrm{~cm}$. high; $\mathrm{C}$, cover of bath, showing openings; $\mathrm{D}$, tube for mineral powder; $\mathrm{E}$, asbestos board cut so as to close central opening in cover. dehydration begins at once and is often nearly finished by the time the thermometerindicates the maximum temperature.

By employing a $U$ tube with glass stoppers, like A, fig. 15, it would be quite possible to ascertain the loss in weight of the powder as well as. the gain of the absorption tube, or to do away with the latter altogether. In this case, however, it would be necessary to. vaseline the stoppers very lightly, and perhaps to introduce the 'powder through a dry funnel because of the vaseline film on the inner side of the end of the tube. The moderate heat on the top of the bath would suffice to prevent condensation of moisture before reaching the absorption tube, or to soon remove what might condense at first, but there must be no long rubber connections.

By employing a suitable oven this form of tube can be used for higher temperatures, but the stoppers must fit air-tight, for vaseline can not be employed.

d. CHATARD'S OVEN FOR TEMPERATURES UP TO $300^{\circ}$ AND OVER.

A form of drying oven devised by Dr. T. M. Chatard ${ }^{a}$ is in use in this laboratory for determining water at different temperatures up to $350^{\circ}$, and gives entire satisfaction. It is an asbestos-covered copper box, B, shown in different aspects and parts in fig. 15. The box is so constructed that the tube with its contents can be removed with-

a Am. Chem. Jour., vol. 13, 1891, p. 110; Bull. U. S. Geol. Survey No. 78, 1891, p. 84. 
out detaching from either the drying or collecting tubes, which is a great advantage if it is desired to apply afterwards the direct heat of a lamp in order to expel the water retained at $300^{\circ}$ to $350^{\circ}$. To facilitate this removal the stand is on rollers, so that after clamping the projecting end of the tube and removing the front of the box, $\mathrm{F}$, and the little side pieces, $\mathrm{S}$, closing the horizontal slits, the oven can be rolled bodily backward, leaving the tube and its attachments in their original position, ready for further heating over a burner or blast.

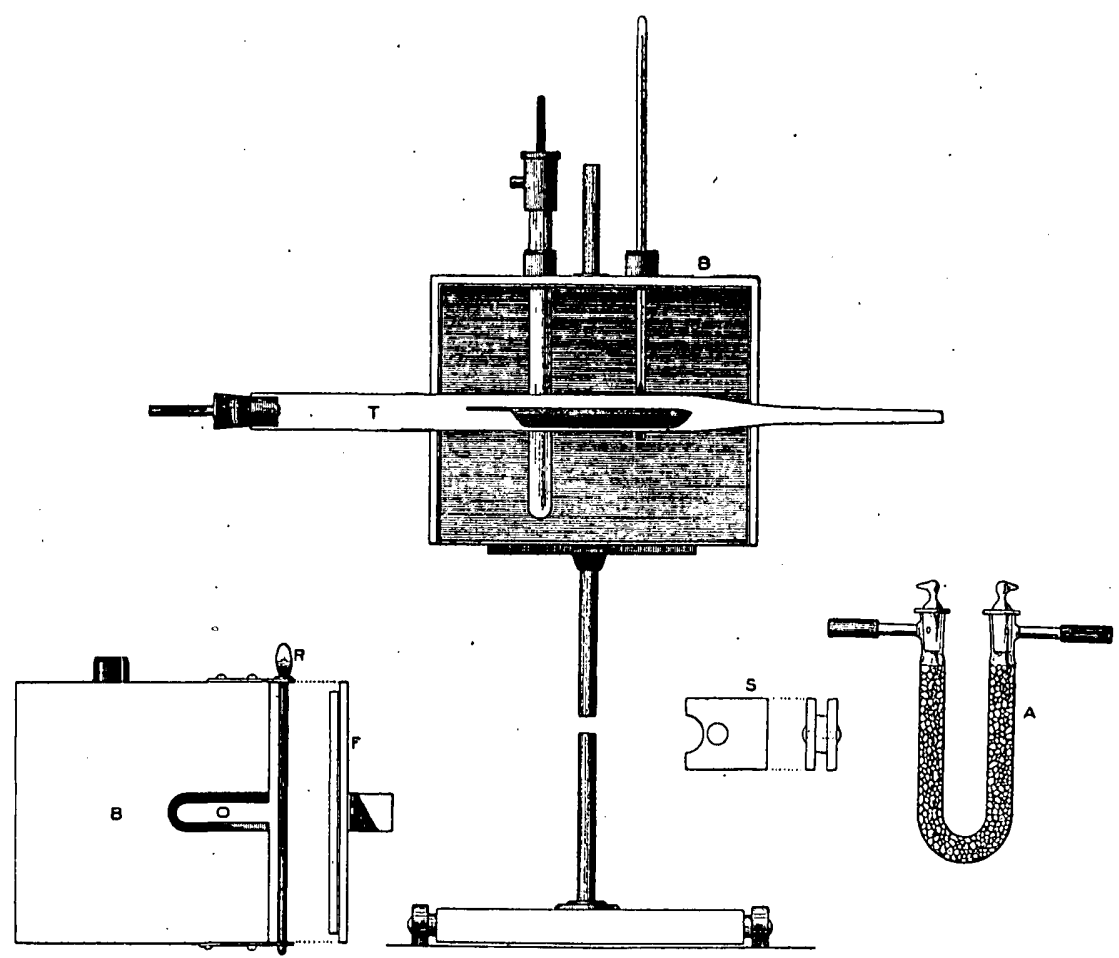

F1G. 15.-Chatard's form of drying oven for water determinations. B, Copper box, $18 \mathrm{~cm}$. long, $10 \frac{1}{2} \mathrm{~cm}$. high, $9 \mathrm{~cm}$. wide, open in front, its sides and top covered with asbestos board; $\mathrm{s}$, two slides of different sizes to close openings, $O$, after the tube is in position; $F$, asbestos-board front stiffened by an interlaid sheet of copper; $\mathrm{R}$, metal rod to hold front in place; $\mathrm{T}$, glass ignition tube; $\mathrm{A}$, calcium-chloride absorption tube. For mineral substances with very high water content the tubes $\mathrm{T}$ and $\mathrm{A}$ may preferably have the original form given them by Chatard, as depicted in Bulletin 176, p. 37. The use of a straight tube, as shown in fig. 15, permits a less complicated construction of box and stand than that here shown, since round holes in opposite ends of the box admit the ignition tube, and the roller base is then superfluous.

The removable front, $F$, of the oven is made of two pieces of sheet asbestos board stiffened by an interlaid piece of sheet copper. The inner piece of asbestos board fits snugly into the box, while the outer one, being slightly larger, by its projecting edges hinders the door from falling in and helps to prevent air currents. This door is held in place by the metal rod, $R$. The little slides, $S$, are made in a somewhat similar manner, and are intended to slip in from the front and close the two openings, $\mathrm{O}$, after the tube is in place, but before closing the front. 
For other forms of tubes adapted to similar determinations, see pages 75 and $82-83$.

e. Gooch's apparatus.

Of more elaborate apparatus, designed to be used with fluxes, the tubulated platinum crucible invented by Doctor Gooch ${ }^{a}$ is capable of affording most excellent service, and it is the one by which far the larger number of water determinations in this laboratory have been and are made.

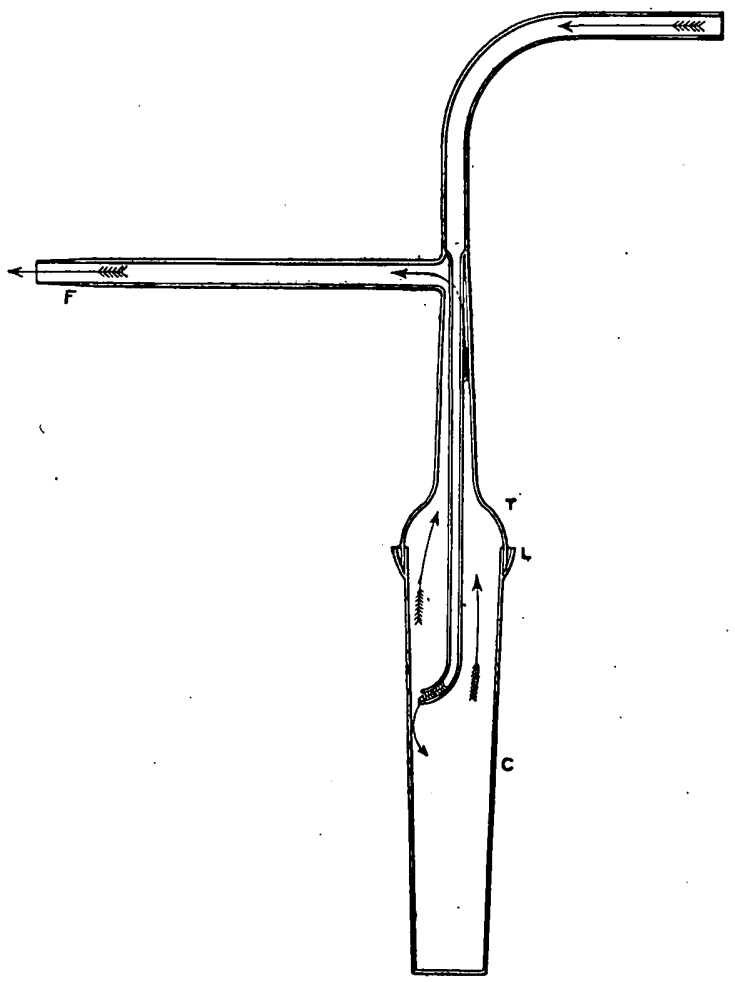

Fig. 16.-Modified form of Gooch tubulated platinum crucible for the determination of water, one-half natural size. Weight about 75 grams.

Fig. 16, which hardly needs detailed description, shows it in a modified form, which differs from the original forms of Gooch in that the tubes for connecting with both the drying and absorption vessels are constructed wholly of platinum instead of lead glass. The form of apparatus depicted in fig. 16 differs from that given in Bulletin 176 by a considerable shortening in length of the inlet and especially the outlet tube, whereby the cost is reduced and attachment can be made to a glass-stoppered $U$ tube, which is the most satisfactory form for absorption tubes. With tubes of the lengths shown in the 
figure there is absolutely no danger of the ends becoming hot enough by conduction to scorch or soften the rubber connection.

As an adjunct to its convenient use there is needed an ordinary. upright iron ring stand, with two small sliding rings, and a sliding ring burner provided with entering ducts for gas and air blast. Across the uppermost ring there is an arrangement of stout platinum wire (S, fig. 17), forming at the center of the ring a secure seat for the upturned flange of the crucible proper. Both rings and burner can be clamped firmly at any height.

The rock powder, having been placed in the cylindrical crucible (C, fig. 16), is there mixed with not more than 3 or 4 grams of fully dehydrated sodium carbonate, ${ }^{a}$ or more of lead chromate if carbon is to be likewise determined. The crucible is sunk in its seat, $\mathrm{S}$ (fig. 17), in the upper ring, $R^{\prime}$, and the tubulated cap, $T$ (fig. 16), is fitted on and attached to the calcium-chloride drying towers-preceded by one containing potassium hydroxide if carbon dioxide is likewise to be estimated-on the one side, and to a sulphuric-acid bulb tube, $B$ (fig. 18), on the other. Powdered sodium tungstate ${ }^{b}$-free from arsenic, which would soon ruin the crucible lips-is now poured into the flanged lip, $\mathrm{L}$ (fig. 16), in which the cap rests, and a metal vessel of cold water having been raised up by the lower ring, $R^{\prime \prime}$ (figs. 17 and 18), until the platinum crucible is sufficiently immersed, the flame of an ordinary blast lamp is turned on to melt the tungstate. As soon as this is fused the flame is removed and the salt solidifies and makes an air-tight joint, the test of which is the permanence of the column of sulphuric acid in the bulb tubes caused by the contraction of the air in the platinum apparatus as it cools.

After drying by a current of air at $105^{\circ}$ for two hours, more or less (see below, p. 81), by means of an air or toluene bath, as shown in fig. 17, the absorption tube, A (fig. 18), is interposed between the sulphuric-acid bulbs and the apparatus, and, while a slow current of air continues to pass, the gradual heating and subsequent fusion of the flux is brought about by the blast-fed sliding ring burner, $\mathrm{R}^{\prime \prime \prime}$ (figs. 17 and 18). The sodium-tungstate joint is shielded from the flame by small pieces of asbestos board, P (fig. 18), cut out so as to fit the crucible. It is well to begin fusing at the top of the mixture in the crucible and to lower the blast gradually till the bottom is reached. In this way may be avoided all danger of the outlet tube becoming stopped by flux carried up by the escaping carbon dioxide.

a This has been heated for a length of time to near its fusing point over a free flame or in an air bath, to decompose the bicarbonate it usually contains, and then placed in a desiccator. Thus heated it is not very hygroscopic. Penfield found that 2.5 grams of it, spread out on a watch glass, gained only 0.0002 gram in fifteen minutes. Potassium carbonate and potassium-sodium carbonate are too hygroscopic by far to be available.

$b$ Private advice from E. W. Morley conveys the information that sodium carbonate is perfectly satisfactory as a substitute for the tungstate. If so, its use is preferable when the contents of the crucible are to be used for other determinations. 
When fusion is complete, as shown in the case of sodium-carbonate flux by the decided slackening of the gas current through the safety bulbs attached to the drying tube, the flame is extinguished and a current of air is allowed to continue until the apparatus is cold.

This apparatus suffers from the drawback of being slightly permeable to combustion gases at high temperature. The defect can be overcome by causing the flame to play upon an outer ordinary platinum crucible, kept permanently filled with sodium-potassium carbonate. This protective crucible, however, is soon ruined for

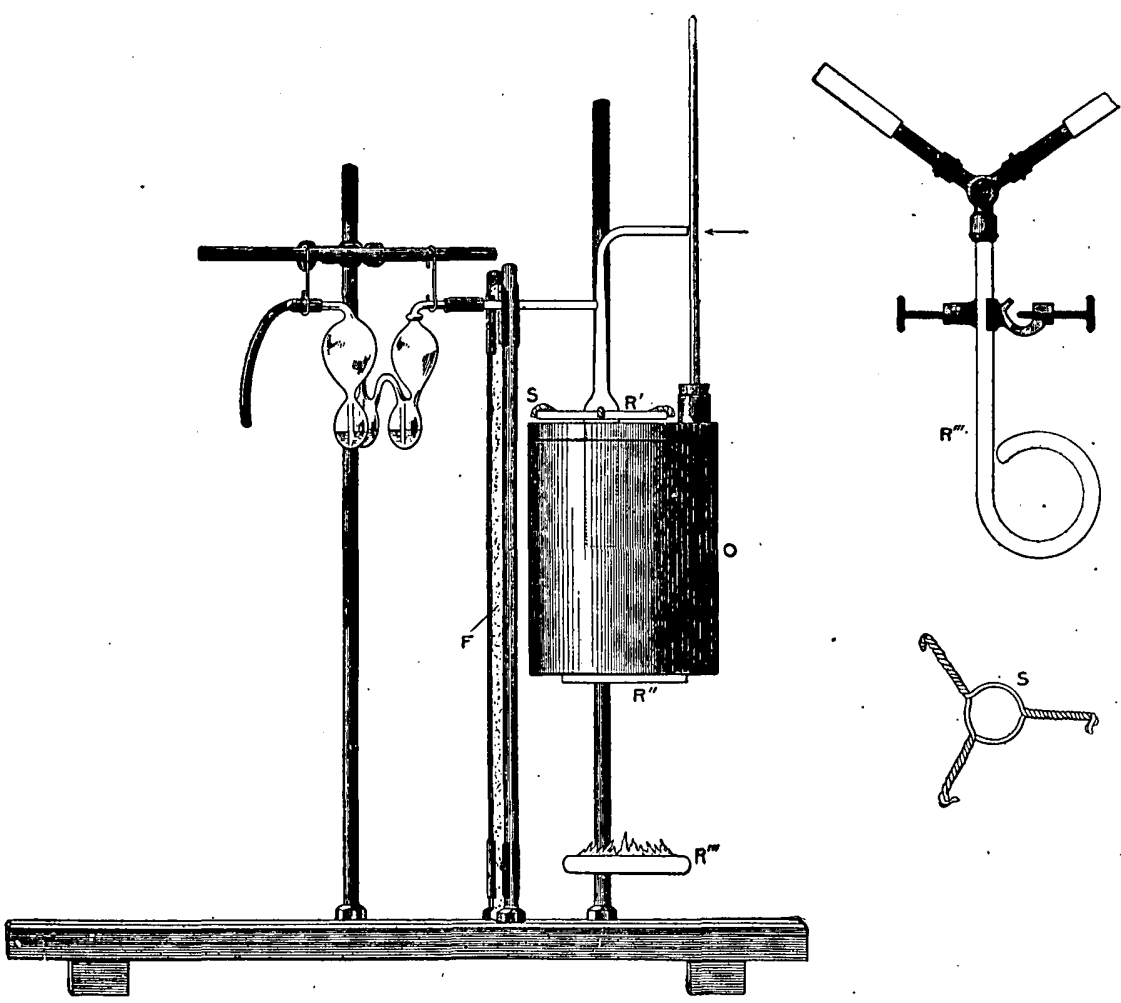

Fia. 17.-A rrangement, during drying, of Gooch apparatus for determining water. S, Seat of stout platinum wire resting on ring $\mathrm{R}^{\prime}$ and serving as a support for the crucible; $\mathrm{R}^{\prime \prime}$, blast-fed ring burner; $\mathrm{R}^{\prime \prime}$, support for air or toluene bath, $O ; F$, asbestos-board shield.

other purposes, being distorted by the alternate expansion and contraction of the carbonate, unless this is poured out before it solidifies after each fusion.

It has been found that if the operation is carried out expeditiously and the final full heat applied for but a few minutes, the error due to penetrating water gases is inappreciable. This hastening may be rendered safer by using rather finely powdered calcium chloride in the central section of the U-shaped absorption tube to avoid large air channels. Through this or any other apparatus based on similar principles the air current should always be forced, not drawn. A 
warm air blast directed upon the exit tube near its entrance into the absorption tube greatly shortens the time required and is to be reconimended.

In this apparatus only the water expelled above $100^{\circ}$ to $110^{\circ}$ should as a rule be determined, and to effect drying of the mixed mineral powder and sodium carbonate, after luting the tubulated cap on the cylindrical crucible with sodium tungstate, the tube is sunk through a round hole in the cover into a small cylindrical air bath

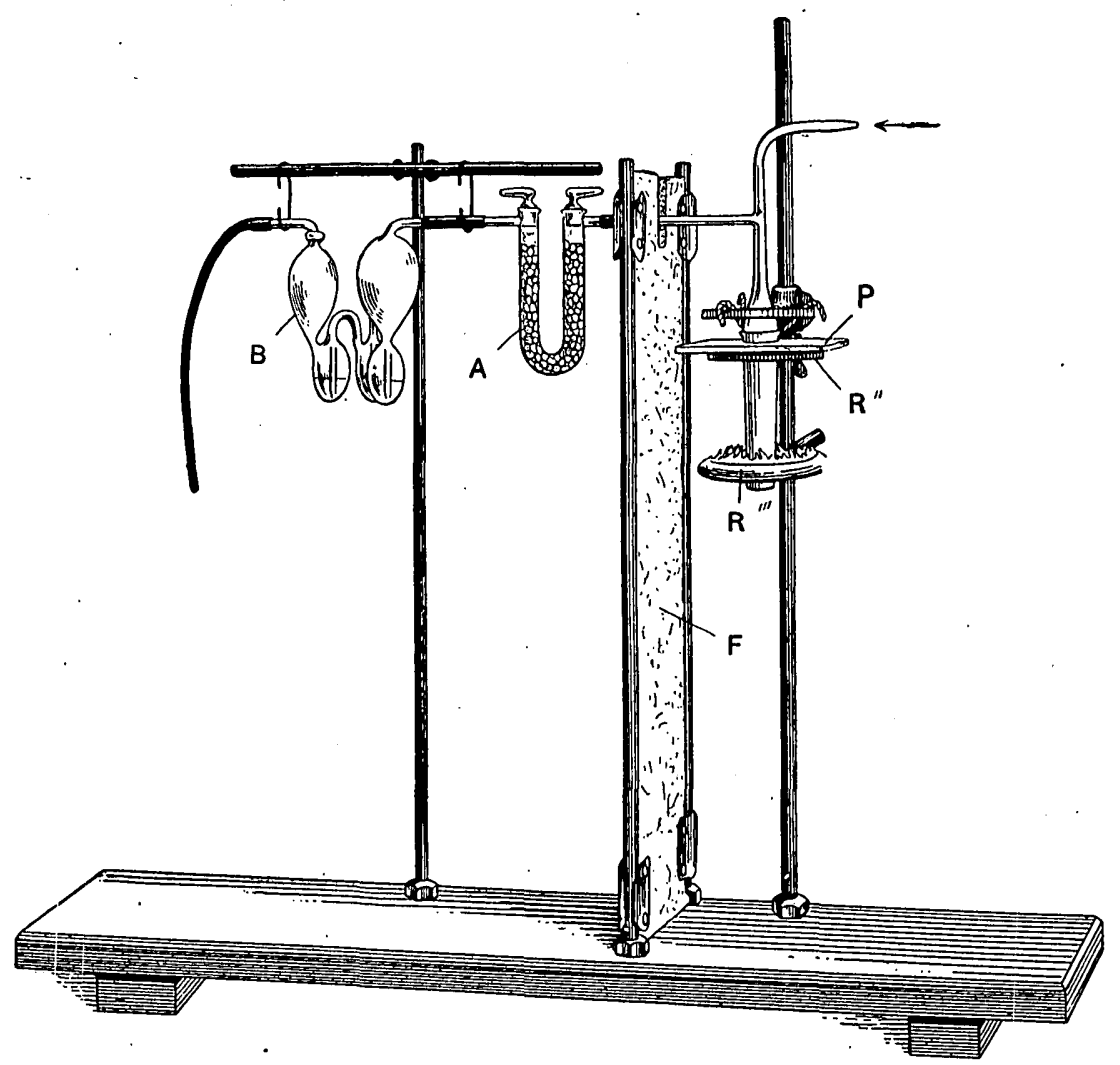

Fia. 18.-Arrangement, during fusion, of Gooch apparatus for determining water. $\mathbf{R}^{\prime \prime \prime}$, Blast-fed ring burner; $P$, protective asbestos shield resting on ring $R^{\prime \prime} ; F$, asbestos-board shield; $\Lambda$, calcium-chloride tube; $B$, sulphuric-acid bulbs serving to show the rate of gas flow through the absorption tube and at the same time to prevent back entry of moisture from the air into $\mathrm{A}$.

(fig. 17), which can be heated from beneath by the same ring burner which is subsequently to fuse the flux. A slow current of air is then forced through and the drying satisfactorily accomplished.

The reason why it is unsafe to attempt estimation of "hygroscopic" moisture in this apparatus is that the luting of the two parts must be done by direct application of a flame to the tungstate, and considerable water vapor may enter the apparatus and be in part retained by the dried sodium carbonate.

20091-Bull. $422-10-6$ 
f. MERITS OF THE ABOVE FORMS OF APPARATUS.

The apparatus described in $b$ and $e$ permit the determination of other constituents besides water in the same portion if necessary, and by the use of lead chromate instead of sodium carbonate, graphite, or the carbon of organic matter, can be simultaneously determined with the water.

To one accustomed to its use, and with a drying and suspension attachment permanently set up, the Gooch apparatus, considering its limitations above set forth, offers perhaps the most handy and convenient means for the determination of water in rocks. Its high first cost, in comparison with the glass tube, is fully made up in time by its durability.

g. JANNASCH'S METHODS.

Jannasch, that zealous deviser of methods for mineral analysis, has published in the Zeitschrift für anorganische Chemie and the Berichte der Deutschen chemischen Gesellschaft sevieral papers dealing with the problem of water determination in minerals, and in his textbook $^{a}$ these are collected in more or less modified form.

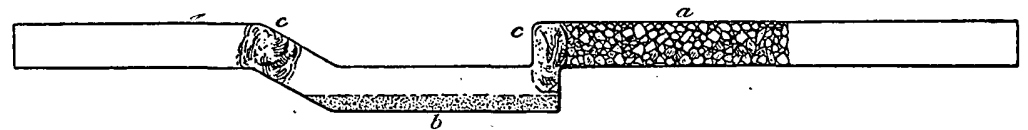

Fig. 19.-Glass tube for determination of water (Jannasch). b, Mixture of mineral powder with borax; $c, c$, plugs of glass wool; $a$, layer of lead chromate or lead oxide. Total length of the tube, $33 \mathrm{~cm}$.; inside diameter, 12-14 $\mathrm{mm}$.

For the majority of silicates he finds dehydrated borax powder a most efficacious flux, usually at a very moderate temperature. The fusion is accomplished either in a platinum boat within a glass tube or in a tube of the form and dimensions shown in fig. 19.

For rocks or minerals containing not much fluorinie à retaining layer of granular lead chromate, or of previously fused and powdered lead oxide, is used as shown at $a$. Plugs of glass wool are used, at $c, c$. Whether or not the boat is employed, the borax is first introduced and, together with the retainer, is thoroughly dried out in an asbestos oven by a hot-air current. Then; after cooling, the mineral powder is added and thoroughly mixed with the borax. Heat is applied by a flat flame to the mixture, which soon melts and forms a clear fusion, when the action is complete. The blast may be used in extreme cases. The layer of retainer must be kept warm by an auxiliary flame, and the absorption tube must be removed before the flame under the fused mass is extinguished, for the glass breaks as soon as this is done. Carbon dioxide can simultaneously be determined by attaching a soda-lime tube to the calcium-chloride 
tube. For one-half to 1 gram of silicate Jannasch uses $1 \frac{1}{2}$ to 2 grams of dehydrated borax.

Regarding the borax method, its inventor insists on the following points as essential to success, especially when the blast can not be applied: Most thorough mixing of flux and mineral powder and a most impalpable fineness of the latter. This last condition is a most serious objection to the method, in view of the effect of fine grinding on the water content of minerals. (See pp. 53 and 64.)

The borax itself is prepared by heating pure crystallized borax in a platinum dish till a small portion has melted. That remaining unfused is powdered and again heated in the dish to dull redness for fifteen minutes, with constant stirring. The powder is placed in a tube with tightly fitting glass stopper and kept over sulphuric acid. It must not be kept long without reheating, because of being hygroscopic.

Another form of tube used by Professor Jannasch for special purposes and recommended by Doctor Dittrich is shown in figure 20. Minerals, such as topaz; which is not fully decomposed by the borax

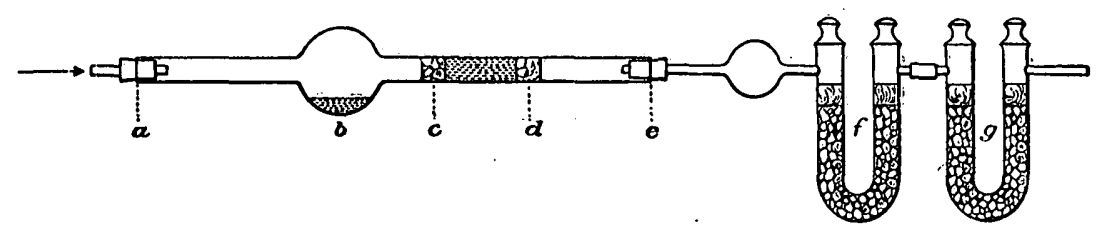

Fra. 20:-Glass tube for determination of water in special cases (Jannasch). Length from $a$ to $e, 26 \mathrm{~cm}$.; inside diameter somewhat over $1 \mathrm{~cm}$; volume of bulb $b, 25 \mathrm{~cm} .^{3} ; c d$, retaining layer of lead oxide between plugs of glass wool; $f$, calcium-chloride absorption tube; $g$, protective tube.

method and which contains a large amount of fluorine, are fused at $b$ with about six times their weight of lead oxide. A layer of lead oxide: (equal parts of oxide and peroxide-Dittrich) between $c$ and $d$ serves to retain any fluorine escaping from the fusion. Doctor Dittrich uses this form generally for substances which can not be heated in the simplest form of Penfield tubes and for those which give off their water on simple ignition, without admixture of lead oxide, though, of course, with the retainer for sulphur, fluorine, etc.

If carbon dioxide is to be simultaneously determined, any carbonate in the lead oxide must be first destroyed by heat. As in the preceding description the layer of retainer must be kept continually hot by an auxiliary flame.

6. SILICA, SEPARATION FROM ALUMINA, ETC.

\section{A. ALTERNATIVE METhods OF DECOMPOSH, THE ROCK.}

a. GENERAL CONSIDERATIONS.

The practice of separating alumina, etc., by the usual methods, after first attacking the rock powder by hydrofluoric and sulphuric acids-silica being estimated in a separate portion-while attractive 
in principle, was long ago abandoned by me after fair trial, owing to the disturbance sometimes occasioned by incomplete expulsion of fluorine ${ }^{a}$ and to a less degree by the presence of sulphates instead of chlorides. With the exception of a comparatively few analyses made thus, the sodium-carbonate method has always been employed. In the case of rocks rich in fluorine strict accuracy would require the separation of silica to be made as in the Berzelius method for fluorine estimation (see p. 184, also footnote, p. 86), but in practice it is not often necessary to resort to this tedious procedure, since the amount of fluorine is usually small and it can by no possibility cause a loss of much more than three-fourths its own weight of silica by volatilization as silicon fluoride when the sodium-carbonate fusion is evaporated directly with hydrochloric acid. Probably the loss is less, since some fluorine perhaps escapes as hydrofluoric acid. However this may be, the error is of comparatively slight importance, since it always attaches to the constituent present in greatest amount.

Various fluxes other than alkali carbonates have been recommended for breaking up silicates insoluble in ordinary acids, such as lead and bismuth oxides, lead carbonate, borax, and boric oxide. Professor Jannasch and his pupils have been especially active in this line of work, as is evidenced by their numerous published papers. One of the advantages most of these fluxes possess over the alkali carbonates is their removability after serving their purpose, thus allowing the various separations to be made more perfectly and without the annoying interference of several grams of foreign fixed salts, which are most troublesome in that part of the analysis devoted to the separation of silica, alumina, iron, lime, and magnesia.

Another of their advantages is that with some of them it is possible to estimate in one portion the alkalies in addition to those constituents usually determined in the silica portion. Where the material is limited, as it so often is in mineral analysis, this is a most important advantage, sufficient to outweigh all possible objections; but in rock analysis, where the supply of material is usually ample, it is rarely worth considering. A still further point in their favor is that it is probably more easy to obtain them entirely free from fixed impurities than an alkali carbonate.

There are, however, objections to their use. With some of them an extraordinary amount of time must be devoted to grinding the mineral to an impalpable powder, with the result of introducing serious analytical complications. (See ante, p. 53.) And the flux itself may need considerable hand pulverization. Once introduced, they

$a$ The presence of fluorine renders the precipitation of aluminum very incomplete. See also Veitch, F. P., Jour. Am. Chem. Soc., vol. 22, 1900, p. 246; also Hinrichsen, F. W., Ber. Deutsch. chem. Gesell., vol. 40, 1907, p. 1497; Zeitschr. anorg. Chemie, vol. 58, 1908, p. 83. 
must be removed before the analysis can be proceeded with, and this removal takes much time and is always a possible source of error.

In mineral analysis these objections are entitled to far less weight than in rock analysis, since the object sought-usually the deduction of a formula-warrants the expenditure of much time and painstaking care. Finally, it has been found that one or more of these fluxes are not available for altogether general use, since certain minerals do not fully succumb to their attack under simple conditions, as andalusite with boric oxide and others with lead oxide (Jannasch). Therefore, however well adapted one or the other of these methods may be for the analysis of homogeneous minerals, it is very improbable that the anticipations of Professor Jannasch, to the effect that the boric-oxide method will soon supersede the alkali-carbonate fusion method in rock as well as in mineral analysis, will be speedily realized Nevertheless, the boric-oxide fusion method, owing to its evident merit, will be described in detail after brief reference to a means of bringing refractory silicates into solution without employing any solid reagent.

The methods of decomposition and the subsequent treatment of the silica hereinafter described are with few exceptions applicable to definite silicate mineral species as well as to complex mixtures of them.

b. DECOMPOSITION OF REFRACTORY SILICATES BY HYDROCHLORIC ACID UNDER PRESSURE. .

Jannasch ${ }^{a}$ pours upon the finely ground rock powder contained in a platinum tube of about $26 \mathrm{~cm} .^{3}$ capacity a somewhat diluted hydrochloric acid (4 acid to 1 water), places over the open end a cap which does not hermetically close the tube, inserts the latter in a larger tube of potash glass likewise partly filled with the diluted acid, seals the glass tube, places it in turn in an inclined position in a steel Mannesmann tube containing ether or benzine to equalize the pressure, and heats to any desired temperature up to $400^{\circ}$.

The chief drawback seems to be a somewhat incomplete decomposition, doubtless due to the necessarily inclined position of the tube, which causes the powder to collect at the lower end, and thus renders decomposition less complete than if the material were spread evenly throughout the length of the tube.. Further, the acid strongly attacks the platinum unless the air in both the platinum and the glass tubes is replaced by carbon dioxide. Fven when this is done, several milligrams of platinum are found in the silicate solution.

Nevertheless, to those possessing the necessary platinum and steel tubes the method can render efficient service in special cases when economy of material is imperative.

a Ber. Deutsch. chem. Gesell., vol. 24, 1891, p. 273; Zeitschr. anorg. Chemie, vol. 6, 1894, p. 72 
c. THE BORIC-OXIDE METHOD OF IANNASCH AND HEIDENREICH. $a$

$\alpha$. Preparation of the boric oxide.-This demands, if the alkalies are to be estimated in the same portion as silica, etc., an absolutely alkali-free boric acid, which can be prepared by two or three recrystallizations of a good commercial article. The purified crystals are dehydrated and fused in a large platinum crucible. This is then suddenly cooled to cause the anhydride to crack into pieces of a size confenient for powdering, which are to be kept in a tight glass and powdered as needed, since the anhydrous oxide is hygroscopic.

B. Treatment of easily decomposable silicates.-To this flux Jannasch and Heidenreich find that nearly all silicates readily succumb over the ordinary blast lamp. The fusion is made in a large crucible holding $40-65 \mathrm{~cm} .^{3}$, and the proportion of flux to be used is gaged according to the nature of the silicate, ranging from 3 to 8 and more parts to 1 of mineral. This last must be finely powdered, especially the most resistant, the authors recommending the expenditure of one-half to one hour's time for the grinding of one-half to 1 gram of powder. A low burner heat is applied for five to ten minutes till water is expelled; the heat is then gradually increased till the gas is fully turned on. Bubbling and rising in the crucible is prevented as far as possible by using a short platinum rod which does not reach above the edge of the crucible. When the mass has been in quiet fusion for a time in the covered crucible the blast flame is applied. The average duration of the entire operation is twenty to thirty minutes, but depends much on the character of the mineral.

$\gamma$. Treatment of refractory silicates.-For those minerals which, like andalusite, cyanite, and topaz, are not fully decomposable by the heat of the ordinary blast flame, Jannasch and Weber ${ }^{b}$ use a flame fed by oxygen instead of air. The blast lamp, of $2 \frac{1}{2} \mathrm{~mm}$. opening, is supplied with gas from at least five or six ordinary gas cocks, and the flame is made broad and free from luminosity. The mineral having been first heated as above described, but with a much larger proportion of flux - as high as 30 to 1 - a few grams additional of boric oxide are added and the oxygen blast is applied till, in ten or fifteen minutes, the fusion is as transparent as glass. ${ }^{c}$

a Zeitschr. anorg. Chemie, vol. 12, 1896, p. 208. This method of decomposing rocks with a view to the determination of their contained alkali originated with Sir Humphrey Davy, as shown in his paper "On a method of analyzing stones containing fixed alkali by means of the boracic acid" (Phil. Trans., 1805, p. 231; Ann. d. chim., vol. 60, 1806, p. 294; Gilbert's Annal., vol. 30, 1808, p. 369; Tulloch's Phil. Mag., 1806, p. 146). Original with Jannasch and Heidenreich is the manner of getting rid of the introduced boric oxide.

$b$ Ber. Deutsch. chem. Gesell., vol. 32, 1899, p. 1670.

c An interesting and important observation reported by Jannasch and Weber is that when the oxygen blast has been used for silicates carrying fluorine or mixed with fluorides the fluorine seems to be wholly expelled as boric fluoride without loss of silica. If this should prove to be generally true, an easy way is at last afforded for determining silica in such cases, where even its detection, when present in small amount, has heretofore been difficult. 
$\delta$. Further treatment after fusion.-From this point the further treatment is the same in both cases and, as modified by Jannasch and Weber, ${ }^{a}$ is as follows:

The hot crucible is cooled in cold water and the contents are turned into a very large porcelain or platinum dish, to which, after covering with a glass, a saturated solution of hydrochloric-acid gas in methyl alcobol is added. ${ }^{b}$ The cover being then removed, the liquid is heated to boiling, over asbestos board, by an inch-high flame, with constant stirring, or it is left without attention over a lower flame or on a water bath heated short of boiling. The crucible is cleansed in a similar manner, and its contents are added to the dish. In ten to fifteen minutes, with occasional addition of the methyl chloride, solution is complete and the liquid is then boiled down to a small volume and evaporated to dryness on the bath. The residue is then digested on a bath at $80^{\circ}$ to $85^{\circ}$ three or four times in succession with the ether solution, in order to remove the last traces of boron as boric ether. Care should be taken to wash down from the sides of the dish, with methyl-chloride solution, the boric acid formed and deposited thereon during the evaporation.

ع. Possible objections to the boric-oxide method.-Very much is clajmed by Jannasch for this method, but with all its undoubted merit there are two points which may militate against it. The boric ether, driven off in such quantities, at once decomposes in contact with moisture, and boric acid settles over all objects with which it comes in contact. The hood must become thickly coated. Hence a special hood for these evaporations alone seems to be called for, otherwise boric acid may at any time fall into other dishes and cause untold trouble. The second objection attaches to the use of the oxygen flame when alkalies are to be estimated in the fusion, and the ability to so determine them is one of Jannasch's chief claims in favor of the method, for it can not be doubted that at the high temperature of this flame alkalies are volatilized. Borax can be slowly but wholly volatilized over the ordinary blast, hence there is great reason to fear sufficient loss at this much higher temperature to give rise to serious error at times.

\section{d. THE SODIUM-CARBONATE METHOD.}

$\alpha$. Advantages of sodium carbonate over sodium-potassium carbonate.-Except in special cases, as when fluorine or chlorine are to be determined, there is no advantage in using the much recommended, because more fusible, double carbonate of sodium and potassium, or the equimolecular mixture of the normal carbonates, or of sodium

a Ber. Dentsch. chem. Gesell., vol. 32, 1899, p. 1670.

$b$ Made by passing dry hydrochloric-acid gas into cooled methyl alcohol for from one to two hours. 
carbonate and potassium bicarbonate. As Dittrich says, ${ }^{a}$ potassium salts are more prone to pass into precipitates than sodium salts and it may be that the higher melting point of sodium carbonate is a distinct advantage. Certainly, for effective decomposition of some rock constituents, a far higher temperature than that of the fusing point of the double salt is required.

$\beta$. Purity of the sodium carbonate.-Notwithstanding the most earnest efforts for years, it has been impossible to procure, either in the open market or by special arrangement with manufacturers, a grade of sodium carbonate which can be called chemically pure. With special precautions small lots can be prepared in the laboratory that will contain less than 1 milligram total impurity in 10 grams; but such an article can not be purchased in the market, and rarely will the so-called chemically pure dry sodium carbonate contain as little as 1 milligram in 10 grams. The invariable contaminating substances-aside from sand and straw, which have sometimes been found-are silica, alumina, iron, lime, magnesia, and sometimes phosphoric oxide, all of these going into aqueous solution with the carbonate. The chief of these impurities are usually silica, alumina, and lime. An article of the above degree of purity is satisfactory in almost all imaginable cases, since the use of the usually extravagant amount of 10 grams for a fusion would introduce an error of but 0.1 per cent in the analysis, supposing 1 gram of mineral to be operated on, and it would, moreover, be distributed over several constituents. This error is undoubtedly fully equaled by the introduction of dust from the air in the various long evaporations.

It is to be borne in mind that the so-called dry sodium carbonate, "C. P." almost always contains some bicarbonate and hence yields water on heating. This, however, in no way detracts from its usefulness, and it is not necessary to convert it wholly to the normal salt for fusions of the kind now contemplated. In fact, C. Holthof ${ }^{b}$ most strongly recommends using the bicarbonate itself as a flux instead of the normal carbonate. (See $\delta$, p. 90.)

$r$. The fusion with normal sodium carbonate.-Ordinarily from 4 to 6 parts of the flux should be used to 1 of rock powder, thoroughly mixed in a crucible of 20 to 30 grams weight. It is inadvisable to use the much larger proportion of flux recommended by some writers, except as it may be shown in isolated instances to be needed. The larger amounts introduce more impurity than the smaller and necessitate longer washing of precipitates. At first the crucible (covered) is placed over a moderately low. Bunsen flame, which is gradually increased to the maximum and maintained there till the mass is quiescent. There should be no violent action. The contents of the 
crucible will then appear, in the case of highly feldspathic or quartzose rocks, as a viscous liquid, occasionally almost clear, though generally more or less turbid, and when placed over the blast little or no further effervescence occurs. Melts of this character disintegrate very readily in water. With less siliceous rocks the fusion is less perfect and may be far from complete, though this does not necessarily imply incomplete decomposition of the silicates. It is generally advisable and often necessary to place these less fusible mixtures over the blast lamp, when a further and very marked escape of carbon dioxide takes place, and the crucible cover should be cautiously lifted at intervals to avoid loss by boiling over. This boiling is due not merely to further action of the carbonate on the rock-forming minerals, but oftentimes more to the decomposition of the alkaline-earth carbonates, whose bases then enter into combination with other constituents of the flux and rock to form compound silicates and probably aluminates. It is a great mistake to regard, as many seem to do, the alkaline-earth metals, magnesium, iron, and manganese as present in the form of carbonates after a blast fusion. They are rarely in that state even when only the Bunsen flame has been used.

The blast flame during fusion should not be directed vertically against the bottom of the crucible, but at an angle against the side and bottom, nor should the flame be allowed to envelope the whole crucible. These precautions apply in all ignitions of reducible substances, and yet they are rarely observed. In neither case, if neglected, will there be the necessary oxidizing atmosphere within the crucible; on the contrary, reduction may occur fraught with serious consequences. This is especially true if the rock contains more than traces of pyrite or other sulphide, when, after cleansing and igniting the crucible, there may appear on its interior a darkening due to oxidation of reduced iron which had alloyed with the platinum. This may in exceptional cases amount to several milligrams in weight, and can. be removed only by repeated ignitions, followed each time by scouring or treatment with hydrochloric acid or acid potassium sulphate. In order to avoid the use of niter in case of pyritiferous rocks, it is well to first roast gently the weighed powder in the crucible in which the fusion is to be made, turning the crucible around a few times to expose all of the powder to the air. With rocks exceptionally high in pyrite the roasting is best done in a porcelain crucible. In such case, after transferring the greater part to the platinum crucible, if brushing with a camel's hair brush does not remove the last of any adhering dust, scouring with a little sodium carbonate will be effective.

- It sometimes happens that the cooled flux, and even its solution, will indicate the absence of manganese when it is really present in quantity to give normally a strong coloration. Two fusions made 
side by side or successively, under apparently similar conditions, may in one case show little or no manganese, in the other considerable. This observation has been frequently made, and therefore the absence of a bluish-green color in the fusion is not to be taken as.a proof of the absence of manganese. This difference of behavior I can ascribe to no other cause than that of a reducing atmosphere in one of the crucibles and an oxidizing one in the other, even though the conditions w.ere apparently alike. It is, of course, not to be expected that the green color can show until all other oxidizible components of the rock, like.sulphides, ferrous iron, and organic matter, have been fully oxidized, which, however, is soon the case if air has access to the surface of the melt.

ס. Fusion with sodium bicarbonate.-C. Holthof, who recommends ${ }^{a}$ this flux strongly, uses 12 to 15 parts of it to 1 of the sample. One quarter of this is placed on the bottom of the crucible, a second quarter is thoroughly mixed with the rock powder in a small warmed dish, and the mixture is then mixed with a third quarter on glazed paper and poured into the crucible. The last quarter is used for rinsing the dish and paper and covering the contents of the crucible, which may be half full. The crucible is heated over a low flame till the bottom is dull red, then during fifteen minutes the flame is increased till the lower quarter of the crucible is dull red, in which state it is maintained for fifteen minutes. Then fusion is brought about by the full flame and maintained for a time. He claims that action between the silicate and carbonate takes place at a lower temperature than with the normal carbonate before fusion, and that for this reason there is almost no spattering onto the lid of the crucible.

$\varepsilon$. Treatment after fusion.-When fusion is complete, the crucible is seized with the tongs (fig. 1, p. 30), and the contents are caused to solidify in a thin sheet over the sides and bottom by imparting an appropriate gyratory motion with the arm during the cooling process. Separation of the mass from the crucible is usually easy, especially if aided by gentle pressure, and much less time is required for its disintegration than when allowed to solidify as a thick cake. If time is no object it is perhaps better to avoid all risk of deforming the crucible and proceed as follows: Let the melt solidify at the bottom of the crucible, first placing in it the bent end of a stout platinum wire. When all is solid, apply the flame again to melt only a thin layer next the crucible, when the cake can be lifted out with ease except for the little that still adheres to the vessel.

The contents of the crucible are placed in a rather tall covered beaker with some water, and hydrochloric acid of 1.1 specific gravity is gradually added in excess. The depth of the evanescent pink color usually produced on addition of the acid allows of judging approxi- 
mately the amount of manganese present. The beaker is placed on the water bath, and when disintegration is complete, having been assisted by gentle pressure with a blunt glass rod, the contents are transferred to a large platinum dish and evaporated on the bath. It is permissible to dispense with the beaker and to perform the whole operation of solution in the platinum dish, but there will be greater solution of platinum in this case by reason of the action of the hydrochloric acid on sodium manganate, etc., in direct contact with platinum.

\section{B. SUBSEQUENT TREATMENT.}

From this point the treatment will ordinarily be the same whether the boric-oxide or the sodium-carbonate method of decomposition has been employed.

\section{a. SEPARATION OF SILICA.}

a. Reasons for adoption of accepted procedure.-The once universal practice of employing a single evaporation with hydrochloric acid for the dehydration of silica has been thoroughly discredited by the work of a number of writers. R. Bunsen ${ }^{a}$ was aware of the impossibility of separating silica by a single evaporation, and he corrected the alumina in his silicate analyses for the silica it was always found to contain. Later, E. Ludwig, ${ }^{b}$ and still later, C. Meineke, ${ }^{c}$ drew renewed attention to the fact and insisted on the importance of correcting the alumina in the manner indicated. Iudwig and, at first, Meineke contented themselves with a single evaporation, and maintained that all of the unprecipitated silica was to be found with the subsequently precipitated iron and alumina. In his second paper Meineke prescribes two evaporations (presumably with intervening filtration) and the rejection of the silica subsequently recovered from the alumina as due to impurity in the ammonia or derived from the vessels, unless these were of platinum. He furnishes quantitative data.

In spite of these publications the practice continued of employing but a single evaporation. Some years later Alexander Cameron, ${ }^{d}$ seemingly in ignorance of the earlier work, reopened the subject with additional quantitative data, and insisted on more than one evaporation. with intervening filtration instead of trying to recover the balance of the silica from the alumina. He also showed, as Ludwig had asserted, that a common practice of evaporating to dryness several times with fresh portions of acid without intervening filtrations did not reduce the silica in the filtrate, and that the presence of aluminum, iron, and calcium was without influence on the results; further,

a Ann. Chem. Pharm., vol. 61, 1847, p. 265

$b$ Zeitschr. anal. Chemie, vol. 9, 1870, p. 321.

c Repert. anal. Chemie, vol. 7, 1887, pp. 215, 757.

$d$ Chem. News, vol. 69, 1894, p. 171. 
that the dehydration was more complete when a high temperature was employed, but that it could not be made complete at one treatment by any modification of the process.

According to J. P. Gilbert, ${ }^{a}$ drying temperatures above that of the steam bath offer no advantage unless much magnesium is present, when the most favorable temperature is $120^{\circ}$. He found that much calcium chloride seems to facilitate dehydration of the silica, but that magnesium chloride above $120^{\circ}$ by decomposing forms a silicate which dissolves in hydrochloric acid and increases the amount of silica carried into the filtrate. He confirmed the earlier belief that drying temperatures higher than that of the steam bath increase the amount of insoluble impurity in the silica, and that this amount can not be overcome by long digestion with hydrochloric acid. Further, he confirmed D. Lindo's statement ${ }^{b}$ that evaporation with sulphuric acid till the appearance of white fumes gives a higher result in silica than with hydrochloric acid. But for general rock analysis the use of sulphuric acid at this stage must be rejected utterly.

Some of the above experiments of Cameron and Gilbert were repeated and confirmed by me. ${ }^{c}$. In the course of this work it was found that hydrochloric acid itself exerts a marked solvent action on silica that has been separated from solution in any of the ways above outlined, whence it becomes plain how a portion of the silica always found in the filtrates gets there, and that it is hopeless to expect to prevent this by a single prolonged drying. Other explanations commonly offered for the observed solubility are the formation of soluble silicates by interaction between the silica and salts present during drying and the protective influence of those salts. The former of these should become more active with increasing temperature, though Gilbert's work does not seem to indicate this except when magnesium is present in quantity.

C. Friedheim and A. Pinagel ${ }^{d}$ prescribe washing the silica with dilute hydrochloric acid instead of hot water because of the marked solubility in water observed by them. But, according to experiments made by myself to test this point, the direction is a vain one, for the results obtained by following it in the ordinary course of rock analysis were worse, if anything, than when pure water was used.

$\beta$. Procedure in absence of notable amounts of fluorine.-Based on the above observations the following procedure is deemed best adapted for general rock analysis, in the absence of more than 0.2 or 0.3 per cent of fluorine.

a Tech. Quart., vol. 3, 1890, p. 61; Abstract in Zeitschr. anal. Chemie, vol. 29, 1890, p. 688.

$b$ Chem. News, vol, 60,1889 , p. 14 .

$c$ Common errors in the determination of silica: Jour. Am. Chem. Soc., vol. 24, 1902, p. 262; Chem. News vol. 86,1902 , pp. 79, 89: For details of experiments referred to here and on several of the following pages the reader will do well to refer to this paper.

$d$ Zeitschr. anorg. Chemie, vol. 45, 1905, p. 411. 
The evaporating dish should be of platinum and as large as possible to permit considerable surface distribution of the dry mass. Those used in the Survey have a capacity of about 1 liter. Porcelain may be used, but never glass; if platinum is not available. Water or stearn-bath temperature suffices, is indeed demanded, for the evaporation to approximate dryness. If the analysis is begun in the morning the first'filtration can be made in the late afternoon. Nothing is gained by continuing the evaporation long beyond apparent dryness this first time, for the slight reduction in the soluble silica gained by so doing is offset by a large loss of time; nor is there much advantage in crushing the residue to powder, whereby, moreover, the dish is easily scratched. The amount of silica passing into the filtrate will generally vary from 1 to 3 per cent of the amount present, and is but little less after twenty hours drying than after one-tenth of that time. ${ }^{a}$ Upon the seemingly dry mass is poured enough strong hyrdochloric acid to thoroughly drench it, $b$ then an equal bulk of water, and the dish is placed covered on the bath for ten to thirty minutes and the contents occasionally stirred. It is quite permissible and sometimes advisable to defer adding the water till the strong acid has been in contact with the salts for ten or fifteen minutes. More water is then added and filtration proceeded with, first by decantation, leaving nearly all the silica in the bottom of the dish. It is generally in a coarse condition and may be ground finer with a pestle. If there is a good deal of iron in the rock it may be advisable to add hydrochloric acid of half strength and heat, after which the silica is brought onto the filter. It is not necessary to remove at this time the film of silica that may adhere to the dish in spots beyond the power of a stiff feather to remove, for in the subsequent evaporation it will disappear. ${ }^{c}$ The washing should be done with cold water or with hot dilute acid till the absence of any yellow color in the precipitate or paper indicates removal of most of the iron. Hot water may with advantage be used for the last washings, and the paper should be sucked dry at the pump.

The filtrate is evaporated again in the same dish to dryness. Usually this state will be reached by the following morning. It may be hastened, if desired, by placing the dish in an air bath at $110^{\circ}$ or $120^{\circ}$. It is probable that a much higher temperature would in the majority of cases have no bad effect, except to increase the amount of insoluble

a See Jour. A m. Chem. Soc., vol. 24, 1902, p. 366; Chem. News, vol. 86, 1902, p. 80.

$b$ E. Jordis and W. Ludewig (Zeitschr. anorg. Chemie, vol 47,1905, p.180) warn against loss by incautious rnoistening of the dry powder with water or acid, especially when these are hot, due to the throwing off of a cloud of dustlike particles. They recommend covering the dish and moistening cautiously with water before adding the acid. With a very capacious dish this is hardly called for.

$c$ Where but a single evaporation is contemplated this film may be removed by warming with ammonia and recovered by reevaporation with acid after driving off the ammonia. (See Kortright, F. L., Chem. Engineer, vol. 5, 1905, p. 19, who gives data to show the very material error ihat often results from neglect to recover this silica.) 
matter other than silica. If the data of experiments 6 to 9 in the paper already $\operatorname{cited}^{a}$ are conclusive, they show that at this stage thorough drying exercises an appreciable effect in reducing the silica in the next filtrate, though the small amounts there reported when experimenting with pure quartz are not to be expected in rock work. If time is no object, and great accuracy is sought, a third evaporation and filtration should be made, for the silica in the second filtrate will amount to 2 to $4 \mathrm{mg}$. Nearly the whole of this can be recovered later from the alumina, but not all, hence the advisability of securing as complete separation here as possible. The second and, if need be, third filtrations with their attendant operations consume less time than the first, and, of course, smaller filter papers are to be used. It is to be noted that these later silicas are more colored than the first, and if the rock is even moderately titaniferous are by no means pure silica.

With minerals like the zeolites, or artificial products such as Portland cements, which are directly soluble in hydrochloric acid, the drying may be very much hastened by placing the covered dish, after bringing to approximate dryness on the bath, on a sand bath or on a triangle which itself rests on a hot plate. The temperature may rise in the dish to $200^{\circ}$; in fact, according to Bertram Blount, ${ }^{b}$ it should not be lower. After an hour's exposure to this temperature less silica will perhaps pass into the filtrate than after prolonged drying at steam-bath temperature, but this is usually at the expense of greater contamination of the filtered silica. Since this contamination is very large (see e, p. 96) with highly titaniferous and ferruginous rocks and minerals, the operator should use discretion in his choice of a high drying temperature.

r. Procedure with rocks and minerals containing fluorine.-This subject has been touched on in commenting on the boric-oxide and sodium-carbonate methods of fusion, ${ }^{c}$ and will be considered in detail under the head of "Fluorine" (p. 184).

b. IGNITION OF SILICA.

The application of a strong blast ${ }^{d}$ for twenty to thirty minutes with crucible covered is absolutely necessary to expel all moisture from the silica, and the latter is then not hygroscopic. The first of

a Jour. Am. Chem. Soc., vol. 24, 1902; p. 306; Chem. News, vol. 86, 1902, pp. 79, 89.

$b$ Jour. Soc. Chem. Ind, vol. 21, 1902, p. 1217.

$c$ See a, p. 83, and footnote, p. 86 .

$d$ It must be borne in mind that some platinum crucibles lose weight steadily and very appreciably on long blasting, not only when new but even after long use. When a crucible suffers from this defect the rate of loss should be ascertained from time to time and allowance made accordingly, or else the wcight of the crucible should be takcn after and not before ignition of the precipitate. (See on this subjcct Hall, R. W., Jour. Am. Chem. Soc., vol. 22, 1900, p. 494, and Hulett, G. A., and Berger, C. W., Jour. Am. Chem. Soc., vol. 26, 1904, p. 1512.) It was hoped that with the advent of the electric furnace prolonged ignitions would not be subject to this source of error, but it was soon found that at $1,250^{\circ}$, the maximum temperature 
these statements, long extant in the literature, has been disputed by $\mathrm{G}$. Lunge and C. Millberg ${ }^{a}$ on the basis of experiments made on silica obtained by the decomposition of silicon tetrafluoride by water. 'It has been shown, ${ }^{b}$ however, that, while the objection holds good for silica thus prepared, it does not for silica obtained by the decomposition of an alkali silicate by means of an acid. F. P. Treadwell has confirmed the observation as to the difference in behavior of the two forms of silica, but regards the blast as unnecessary if a Teclu instead of a Bunsen burner is used. My experience is not in agreement with this. ${ }^{c}$

Although the above time limit usually suffices with a powerful blast, and may be materially shortened for small amounts of silica, such as are usually found in limestones and even in cements, there is need occasionally to prolong it in very exact work until repeated weighings give the same value or a loss no greater than that suffered by the crucible itself. ${ }^{d}$

\section{CORRECTION FOR IMPURITIES IN THE SILICA.}

The weight of the crude silica should always be corrected for impurities, which are never entirely absent, by evaporating with sulphuric and hydrofluoric acids and again blasting for a minute or less, according to the amount of residue. It is a mistake to neglect another weighing at this point, even if the residue may appear insignificant. In the author's experience it never has been found to be unweighable, and, furthermore, the error due to loss in weight of the crucible itself is eliminated if the weighing is made. The silica in the

attainable in the furnace at the Survey, medium-sized crucibles lost approximately 1 milligram in weight per hour. Similar losses have since been noticed and published by others. Their cause seerns to be chiefly the distillation of iridium out of the alloy: $\Lambda$ t one time an error of about the same magnitude in the opposite direction caused perplexity until it was found that the platinum wire in the bedplate of the furnace was burned out. The crucible, then occupying the coolest place in the furnace, received a deposit of some of the inetal volatilizing from the wires in the side plates. Iron has also been found recently in platinum ware in large amount (see p. 41).

a Zeitschr. angew. Chemie, 1897 , p. 425 .

$b$ Hillebrand, W. F., Jour. Am. Chem. Soc., vol. 24, 1902, pp. 372-373; Chem. News, vol. 86, 1902, p. 91. c It was thought that the observation reported by E. Jordis and E. H. Kanter (Zeitschr. anorg. Chemies vol. $35 ; 1903$, p. 16), to the effect that artificially prepared silica when digested with hydrochloric acid take, up chlorine and holds it in a chemical combination that is only incompletely broken up on ignition, might have bearing on this point. To test this, precipitated silica was fuscd with sodium carbonate, the melt was dissolved in water and evaporated with hydrochloric acid as in silicate analysis, and the separated silica was washed with cold water till free from all but a hardly detectable trace of chlorine (wash-water test) and dried at gentle heat. Instead of the large amount of chlorine reported by Jordis and Kanter, this silica yielded only the minute trace that blank tests with the reagents afiorded, and sodium in weighable amount was also absent. Evidently, as stated by Jord is and Kanter, the chlorine compound is readily decomposed by water. The tests for chlorine were made by dissolving the silica in presence of silver nitrate in hydrofluoric acid free from chlorine, also by fusing with chlorine-free sodium carbonate, dissolving in water, acidifying with nitric acid, and then adding silver nitrate.

$d$ For the proof see Hillebrand, W. F., Jour. Am. Chem. Soc., vol. 24, 1902, p. 373; Chem. News, vol. 86, 1902, j). 91. The duty of every analyst to test the efficiency of his blast or muffle can not be too strongly emphissized. The discordance of many statements and results on a variety of subjects is to be attributed to a difference in the ignition temperature employed by different writers. A rcally good blast will readily melt $a_{\text {s }}$ few centigrams of orthoclase powder in the bottom of a 15-gram platinum crucible, showing a temperature of probably $1,200^{\circ}$. 
crucible should be moistened with water before adding the hydrofluoric acid, though with strongly blasted silica the action is not so violent as with that which has not been thus treated. With rocks carrying 60 to 80 per cent of silica one or two drops of sulphuric acid diluted one-half suffice, but with less siliceous rocks the amount ought to be increased, not only in order to surely afford acid enough for the conversion of all the contaminating bases to sulphates, but also to prevent loss of titanium by volatilization as fluoride, since with increasingly basic character of the rocks the percentage of this element in the residue is likely to increase.

Precipitated silica is easily removed by one evaporation with a sufficiency of hydrofluoric acid, but if quartz happens to be present this is not the case, for it is but slowly dissolved and more than one treatment may be necessary.

The subsequent precipitate of alumina, etc., is usually ignited in the crucible containing the residue from the silica.

\section{d. ACCURACY OF THE SILICA DETERMination.}

By observing the above rules-use of platinum, repeated evaporations and filtrations, proper blast ignition, and correction for foreign matter-the determination of silica, from being one subject to grave error, has become one of the most exact of which I have cognizance, provided precautions are taken to recover, as hereinafter described, the portions that inevitably are to be found with the alumina. With careful work variations of 0.1 per cent are the exception in duplicate analyses.

e. COMPOSITION OF THE RESIDUE OBTAINED FROM THE SILICA.

The qualitative composition of this residue varies with different rocks less than its quantity. It will contain alumina and ferric, titanic, and phosphoric oxides invariably if these are present in the rock. If the rock is low in the last three, particularly the last two, the residue should be slight, occasionally less than $1 \mathrm{mg}$. It is usually much greater, and may amount to 2 or even 3 per cent with basic rocks very rich in titanium and phosphorus. It is, however, a great mistake to suppose, as some chemists still seem to, that all or nearly all the titanium is to be found with the silica. It may reach onethird of the total amount.

If in correcting the silica, toward the end of the evaporation, when the hydrofluoric acid has been driven off and the sulphates begin to appear in solid form, the residue has a peculiar milky or enamel-like appearance, it may be taken as evidence of much phosphorus and titanium, though possibly the appearance may be due to zirconium with the other elements named. Although observed in but one series 
of rocks, ${ }^{a}$ the appearance is so unusual and striking that it was deemed worthy of record.

It might be supposed that the residue would contain most of the barium of those rocks carrying that element together with sulphur or sulphates, but in my experience this is not so. Only when there is considerable excess of $\mathrm{SO}_{3}$ over $\mathrm{BaO}$ will any of the latter be present in the residue, and in the vast majority of cases there is none at all. This is due to the appreciable solubility of barium sulphate in hot hydrochloric acid, coupled with the fact that barium is so minor a constituent of most rocks. Should some of it be present in the residue, its removal and estimation at this stage are not necessary, as it can be more conveniently recovered later, together with the silica accompanying the precipitate of alumina, etc. (f. $\beta$, p. 107).

Quite as rarely is calcium ever a component of the residue if the decomposition of the rock powder was complete at the outset. I have frequently found that the residue, after re-solution, is quantitatively precipitable by ammonia in presence of an ammonium salt. W. R. Bloor, ${ }^{b}$ however, in analyzing clays, invariably found calcium and magnesium as well as alkalies in this residue in amounts that were surprisingly large, considering the relatively small percentages of these elements in clays: I have since tested the matter carefully as to celcium and magnesium, finding none of the former and but 0.2 to $0.3 \mathrm{mg}$. of $\mathrm{MgO}$, when present at all.

\section{f. Platinum in filtrates.}

The filtrates from the silica always contain notable amounts of platinum. This arises in very small degree from the crucible fusion, unless niter was added; in a larger degree indirectly from the action of hydrochloric acid on the manganate, vanadate, and sometimes chromate of sodium; and, if much iron is present, in no small degree from the reduction of ferric chloride to ferrous by the platinum of the dish. This last reaction has attracted little attention until recently, but is mentioned by Gmelin-Kraut, ${ }^{c}$ and can be readily demonstrated by evàporation of ferric chloride in platinum.

The removal of this platinum before precipitating alumina and iron is not necessary (but see third footnote, p. 107), and to do so involves the reoxidation of all iron and subsequent boiling to remove or destroy the excess of oxidizing agent, together with the expenditure of much valuable time. The iron is already oxidized by the fusion, and needs no further help in that direction. Nevertheless, if time is not a prime object, its removal by hydrogen sulphide is to be recommended. In

$b$ Jour. Am. Chem. Soc., vol. 29, 1907, p. 1603.

c Anorg. Chemie, 6th rev. ed., vol. 3, 1875, p. 359. 
the following descriptions, however, it is assumed that the platinum has not been gotten rid of at this stage.

\section{METALS PRECIPITABLE BY HYDROGEN SULPHIDE.}

The presence in appreciable amounts of metals precipitable by hydrogen sulphide, except perhaps copper, is of so infrequent occurrence in most rocks that discussion is unnecessary in their connection. In case it is necessary to precipitate them at this stage, however, it is always well to bear in mind that a little titanium may be thrown down along with them. Separations of the silica should be made in porcelain, to eliminate platinum; or, better still, the quantitative determination of these metals should be made in a separate portion of the rock broken up by the action of hydrofluoric and sulphuric acids.

The warning may here be repeated that the samples should never be passed through copper or brass sieves when copper or copper and zinc are sought.

\section{ALUMINUM-TOTAL IRON.}

\section{A. INDIRECT METHOD FOR ALUMINUM (TITANIUM).}

\section{a. PRELIMINARY REMARKS:}

The difficulties are very great in the way of successfully producing and handling the complex precipitate which is obtained in the next stage of the analysis and which should contain the aluminum, iron, titanium, and phosphorus, besides chromium, vanadium, zirconium, and rare earths if present, and according to some preferences the manganese. It is fully recognized that there are objections to parts of the procedure that is usually followed in this laboratory, but the same may be said of any other known procedure, including that preferred by Dittrich. It is believed that the Survey method, which in its main features is closely modeled after old and well-known methods, will in competent hands give better results on the whole than any other.

The common practice in this laboratory is to find alumina by difference, after deducting from the precipitate produced by ammonia or sodium acetate the sum of all other oxides this precipitate may contain. Of these, only ferric oxide, titanic oxide, and the trace of silica are determined in this portion (see also third footnote, p. 107), those of phosphorus, vanadium, chromium, zirconium, and sometimes manganese being looked for in other portions of the rock powder. This throws on the alumina all errors involved in their separate determinations; but these errors may balance, and in any case the probable error can hardly be as high as that involved in the direct weighing of the alumina itself, considering the difficulty of effecting a satisfactory 
separation of it from all the other admixtures, an operation which would, moreover, immoderately extend the time required for each analysis.

b. PRECIPITATION OF ALUMTNUM, IRON, ETC., WITHOUT PRECIPITATING MANGANESE.

$\alpha$. Precipitation by ammonia.-Two precipitations by ammonia at boiling heat are usually quite sufficient to separate iron, aluminum, ${ }^{a}$ phosphorus, vanadium, chromium, titanium, and zirconium, if all these are present, from nickel, manganese, the alkaline-earth metals, and magnesium, provided ammoniacal salts are present in sufficient quantity. This last point is of special importance as regards magnesium, and failure to observe it is doubtless the reason why many old analyses, and sometimes modern ones, show utterly improbable percentages of alumina, especially as chemists were formerly often satisfied with a single precipitation. The necessary ammonium chloride is better obtained by the use of purified ammonia water and hydrochloric acid than by the addition of the solid salt, which is seldom pure. The chemist should satisfy himself what amounts of ammonium salt are needed to hold in ammoniacal solution the maximum percentage of magnesium that the rock he is analyzing might contain. With extremely high magnesian rocks a third precipitation is generally requisite, and a minute amount of calcium will sometimes be found in the third filtrate.

The precipitations are made in a bulk of 300 to $400 \mathrm{~cm}^{3}$, at boiling heat, in a platinum dish if possible, otherwise in one of the borosilicate beakers of the Jena or Nonsol type. The ammonia should be as free from carbonate as possible, as well as from any nonvolatile matter, and it need not be added in any great excess. The complete boiling off of this excess is unnecessary, as pointed out by Genth and Penfield, since it is apparently the washing with pure water and not the free ammonia which carries small amounts of alumina into the fil-

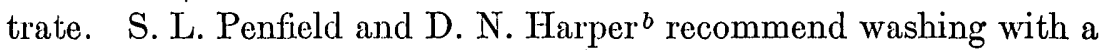
dilute solution of ammonium nitrate $\left(20 \mathrm{~cm} .^{3}\right.$ nitric acid, neutralized by arnmonia, to the liter), and also the solution of the first precipitate in nitric instead of hydrochloric acid, in order to shorten the washing, there being no chloride to remove, both of which are excellent suggestions. ${ }^{c}$

\footnotetext{
a $\mathrm{I}+$ is well to bear in mind that in presence of fluorine, aluminum is very incompletely precipitated by ammonia. Consult also Veitch, F. P., Jour. Am. Chem. Soc., vol. 22, 1900, p. 246; and Hinrichsen, F. W., Ber. Deutsch. chem. Gesell., vol. 40, 1907, p. 1497, and Zeitschr. anorg. Chemie, vol. 58, 1908, p. 83.

$\checkmark$ A.m. Jour. Sci., 3d ser., vol. 32, 1886, p. 112.

$c A$ single objection, and this of a practical nature, weighs against these suggestions. It will not do to evaporate to dryness filtrates containing both chlorides and nitrates in the same platinum dish, still less to drive off the mixed ammoniacal salts in platinum. With much iron present the final precipitation from a nitric-acid solution is a decided advantage, but is less so in the case of alumina free or nearly free from iron. Contrary to numerous statements and general belief, there is no danger of loss of alıminum chloride by volatilization when the precipitate is ignited before complete removal of ammonium chloride.
} 
For a reason mentioned under e (p. 104) the final precipitation may with advantage be made in the presence of macerated filter paper, as recommended by Dittrich. ${ }^{a}$

In the presence of little or no manganese, and also in the absence of iron, the ammonia method of precipitation is preferable by far to the basic-acetate method described in the following section, but it will occasionally happen that the separation from even very small amounts of manganese is altogether incomplete, and the uncertainty of insuring this separation led me to employ the basic-acetate method for the first precipitation in all cases where manganese is present-and the exceptions are few-even though the precipitation of alumina is sometimes less complete than by ammonia and in spite of other. admitted defects, as, for instance, a tendency of the precipitate to run through the filter on washing. ${ }^{b}$

The most likely explanation of the persistent retention of small amounts of manganese by the precipitates produced by ammonia or acetates, even after repeated precipitation, seems to be that a portion of the manganese becomes oxidized to the $\mathrm{Mn}_{2} \mathrm{O}_{3}$ state and remains so when redissolved, being then in a fit condition for reprecipitation.

$\beta$. The basic-acetate precipitation.-Although for this precipitation most writers seem to prescribe or prefer sodium acetate, except when the presence of a fixed alkali would interfere with subsequent operations, there does not seem to be any valid reason why ammonium acetate should not do quite as good service, besides having the advantage of easy removability afterwards in case of need. Also there is probably no good reason why the corresponding carbonates instead of the hydroxides should be used for neutralizing the hydrochloric solution before adding the acetate other than the fact that they were, when the methods originated, and still are, easier to obtain comparatively free from silica and alumina. It is to be borne in mind that with precipitates low in iron and high in aluminum the separations are less satisfactory than when the reverse condition prevails. In rocks, alumina usually predominates, of ten largely, over iron oxide, so that none of the methods of making the basic-acetate separation that involve the presence of notable quantities of free acetic acid are permissible, because of the solubility of aluminum hydroxide in this acid even when it is very dilute.

The cold and not excessively acid solution, not exceeding $100 \mathrm{~cm}^{3}$ in bulk and contained in a beaker of Jena or Nonsol glass, is first neutralized with great care by adding a freshly prepared solution of

a Anleitung zur Gesteinsanalyse, 1905, pp. 10, 11, 14; Ber. Deutsch. chem. Gesell., vol. 37, 1904, p. 1840.

$b$ The fact must not be overlooked that certain of the rare earths may pass completely into the filtrate if the basic-acetate method is followed. If, then, later, on rendering the combined filtrates ammoniacal, an unexpectedly large precipitate appears, this should be carefully examined as to its nature. In an analysis of piedmontite from Maryland over 2 per cent of rare earths, including cerium and others not identified, were quantitatively separated in this way from iron, alumina, etc. 
sodium carbonate drop by drop. When the solution begins to deepen in color, the addition of the carbonate is continued with less speed and with longer stirring between the successive drops. When, now, the precipitate that each drop causes begins to dissolve very slowly, no further addition may be made till the liquid becomes quite clear again.' With practice, it is not difficult to recognize this condition, even when the liquid is very dark. The intensity of the color will depend on the amount of iron present and the degree of dilution; therefore with small amounts of iron one is much more liable to overstep the end point than with solutions rich in iron. When, in spite of vigorous stirring, the turbidity after a final drop'seems rather to increase than diminish, one or, if need be, two drops of acid are added. If this does not clear the liquid, it is best to add a slight excess and to repeat the neutralizing process.

T.wo or three grams of sodium acetate dissolved in a small amount of water are now poured in and then boiling water till the total bulk is about $400 \mathrm{~cm} .{ }^{3}$. The beaker is placed over a lamp and its contents brought to boiling and kept in that state for two or three minutes. As soon as settling has taken place filtration is proceeded with. It is generally best to dispense with the pump at this stage and to use a filter of such size that the whole of the precipitate can be conveniently brought upon it without filling to the top. The washing is to be done with hot water, to which has been added a very little sodium acetate for the purpose of guarding against an otherwise inevitable turbid filtrate.

The precipitate, having been sucked dry at the pump, is redissolved in hydrochloric acid, reprecipitated by ammonia as in $\alpha$, washed, sucked dry, and treated as in e (p. 104), after recovery as in d (p. 103) of the portions of aluminum and iron that may have passed into the filtrates. ${ }^{a}$

\footnotetext{
a.Mittasch's method for the basic-acetate separation of manganese from iron.- $A$ study of the conditions necessary for successful separations of large or small quantities of manganese from iron by one treatment has been made by A. Mittasch (Zeitschr. anal. Chemie, vol. 42,1903, p. 492), who gives the following directions for the use of ammonium acetate. It must be borne in mind, however, that this method is not applicable to aluminous materials.

'The solution containing not much free acid (hydrochloric) and preferably cool, whose volume should not: exceed $100 \mathrm{~cm} .{ }^{3}$, is neutralized in a beaker with concentrated ammonium-carbonate solution (200 grams per liter, about double molar) till the precipitate that is formed begins to redissolve slowly, then with weaker solution (about one-tenth molar) till a slight precipitate persists for one or two minutes in spite of stirring. Next is added, according to the amount of this precipitate, 3 to $5 \mathrm{~cm} .^{3}$ of acetic acid (double normal) if the commercial article of ammonium acetate, which nearly corresponds to the acid salt ( $\left.\mathrm{NH}_{4}\right)$ $\mathrm{C}_{2} \mathrm{II}_{3} \mathrm{O}_{2} \cdot \mathrm{C}_{2} \mathrm{H}_{1} \mathrm{O}_{2}$, is used, or $10 \mathrm{~cm} \cdot{ }^{3}$ if the normal salt is used. This last is made by neutralizing acetic acid with ammonia. The solution is then diluted with water to about $400 \mathrm{~cm} \cdot{ }^{3}$ and brought nearly to the boiling point. Any precipitate that may have appeared being disregarded, $20 \mathrm{~cm} . .^{3}$ of the acid acetate (one-half molar) or $5 \mathrm{~cm} . .^{3}$ of acetic acid and $10 \mathrm{~cm} . .^{3}$ of the normal acetate (molar) is added, and the boiling temperature is maintained for a minute longer. Precipitation is complete even at $60^{\circ}$, but filtration is easier if the boiling temperature is reached. As soon as the solution is settled filtration is begun and the washing performed with hot water at first, then with hot water containing some acetate and a little acetic acid. Any adhering film is removed from the glass with hydrochloricacid and reprecipitated with ammonia. If this is added to the main filter it must not be done till the large precipitate has been washed completely, because of the danger of precipitating, by the ammonia, manganese that has not yet been washed out.

The Brunck-Funk separation of iron as basic acetate from manganese, zinc, nickel, and cobalt.-The followIng method of O. Brunck (Chem. Zeitung, vol. 28, 1904, p. 514), slightly modifed and extended by W. Funk
} 
c. PREcipitation of iron, aluminum, etc., together With MANGanese.

a. By ammonia and ammonium persulphate.-This method has for its object the coprecipitation of manganese with a view to simplifying subsequent operations. It is, however, defective in case the rock carries appreciable amounts of barium and strontium or is very high in calcium, by reason of the certain partial precipitation of some of one or both of the first two as sulphates and its possibility in the case of calcium. It is probable that calcium can be completely held in the filtrates by two precipitations in analyzing almost any silicate rock, but this is not at all certain with respect to limestones, a point that has not yet been investigated. If neither strontium nor calcium is present in sufficient amount to be retained in the precipitate, there is no vital objection to the method on this score, for barium can be recovered, as in $\mathrm{f} . \beta$, page 107 .

The applicability of the method as above limited being assumed, it may be carried out as follows, though too few tests have thus far been made in this laboratory to warrant its unqualified recommendation.

To the filtrate from the silica contained in a platinum dish, and in bulk from 100 to $200 \mathrm{~cm}^{3}$, is added enough hydrochloric acid to prevent precipitation of manganese when made ammoniacal (see b. $\alpha$, p. 99). Heat is applied, and about 1 gram of ammonium persulphate ${ }^{a}$ free from all interfering impurities is added. When the liquid begins to boil, ammonia is added carefully till in decided excess, as shown by the odor, and boiling is kept up for several minutes. The precipitate is allowed to settle and is quickly filtered and washed with hot water three or four times and sucked dry by the pump. It has

(Zeitschr. anal. Chemie, vol. 45,1906, p. 181) is claimed to afford a complete separation by one treatment. Its applicability to aluminous materials has, however, not been tested, and it is quite improbable that it would work with rocks containing phosphorus and titanium.

The method differs from other modifications of the acetate process in the avoidance of the delicate neutralization, by removing excess of acid by evaporation and in the addition of potassium chloride to form a double salt with ferric iron, and thus prevent the separation of a basic chloride during the drying. As given by Funk for materials soluble in acids the procedure is as follows:

To the chloride solution in a large dish is added potassium chloride (about 0.35 gram to 0.1 gram of iron) the solution is evaporated to apparent dryness on the steam bath, but without expelling all of the free acid. The residue is broken up superficially and the drying on the bath continued for five to ten minutes. It is then taken up in 10 to $20 \mathrm{~cm} . .^{3}$ of cold water, with which it should give a clear solution. It is requisite that there shall remain a slight amount of free acid to set free acetic acid from the acetate in the next stage of the operations. One and a half to two times the theoretically needed quantity of sodium acetate (3 molecules of $\mathrm{NaC}_{2} \mathrm{H}_{3} \mathrm{O}_{2} \cdot 3 \mathrm{~F}_{2} \mathrm{O}$ to 1 atom of iron) is now added, in a solution which has been rendered slightly acid with acetic acid if originally alkaline. After diluting with cold water (to 400 to $500 \mathrm{~cm} . .^{3}$ for 0.2 gram iron) the solution is gradually heated with stirring to the point of precipitation $\left(60^{\circ}\right.$ to $\left.70^{\circ}\right)$, allowed to settle, decanted, collected on the filter, and washed with hot water.

$a$ This reagent as sold must be purified. Jannasch (Prakt. Leitfaden d. Gewichtsanalyse, 2d ed., 1904, p. 179) accomplishes this as follows: To a saturated warm solution (not over $95^{\circ}$ ) ammonia is added till its odor persists. [More will have to be added at intervals, since the continual decomposition of persulphate sets free sulphuric acid.-W. F. H.] When the precipitate of alumina, etc., has settled, the solution is filtered as rapidly as possible through asbestos, a porcelain funnel being used, since the strong solution attacks both paper and glass markedly. When cooled to about $30^{\circ}$, the clear filtrate is poured into twice its volume of alcohol and further cooled by ice applied externally. After half an hour the precipitated persulphate is collected on a filter, washed with alcohol, and then with ether, and dried in the air. The alcohol must be thoroughly removed. [ A commercial article purified thus by me was found to contain no interfering impurities, though not free from alkali.-W. F. H.] 
not been determined if such a very dilute solution of persulphate has any appreciable effect on glass, but apparently not, to judge from my own limited experience. The precipitate is then dissolved in a small amount of hot hydrochloric acid containing a few drops of sulphurous acid, and after addition of well-macerated filter paper reprecipitated precisely as the first time. It may then be washed three or four times with hot water containing a few drops of ammonia, then ignited in the paper, and weighed as $\mathrm{Al}_{2} \mathrm{O}_{3}, \mathrm{Fe}_{2} \mathrm{O}_{3}, \mathrm{Mn}_{3} \mathrm{O}_{4}, \mathrm{TiO}_{2}$, and $\mathrm{P}_{2} \mathrm{O}_{5}$. Any chromium in the rock should be in the filtrates, wholly oxidized by the persulphate, but it will be recovered with the unprecipitated traces of alumina, as detailed under d. $\gamma$, page 104, and should be added to the main precipitate above. It is not best to attempt to determine the chromium colorimetrically in the filtrate.

B. By ammonia and ammonium sulphide.-Although this old method of precipitation can be employed in special cases, it is not well adapted generally to silicate rocks, and will, therefore, not be described in this connection.

\section{d. RECOVERY OF IRON AND ALUMINUM FROM THE FILTRATES.}

$\alpha$. After an ammonia precipitation.-The filtrates are evaporated, always in platinum if possible and allowable, to a small volume. If ammonium salts are present in abundance, it is advisable to evaporate the filtrates separately, the second to dryness. Its dry contents, almost wholly ammonium salt, are expelled by heat, the slight residue being taken up with hydrochloric acid and added, whether wholly soluble or not, to the other filtrate reduced in bulk. To this ammonia is now added and the evaporation continued, the solution being kept alkaline, in order to coagulate the small amount of alumina (and usually iron hydroxide) that will invariably be found. This is collected on a small filter and the filtrate caught in a flask of 150 to $200 \mathrm{~cm}^{3}$ capacity, if manganese is to be determined, otherwise in a platinum dish of suitable capacity for the calcium precipitation. The small precipitate is washed with hot water, burned, and added to the main one.

3. After a basic-acetate precipitation.-The first filtrate is evaporated to, or nearly to, dryness, in order to get rid of the excess of acetic acid. The residue is then taken up, with only enough hot water to keep it in solution, and the dish is replaced on the bath for a short time. The precipitate, which will generally be appreciably larger than that obtained in $\alpha$, is collected on a small filter ( $7 \mathrm{~cm}$.), and the filtrate in a flask of 150 to $200 \mathrm{~cm} \cdot{ }^{3}$ capacity. The second or anmoniacal filtrate, having been separately evaporated to small bulk, with the addition of a few drops of ammonia toward the end, serves as first wash water for the other dish and for the precipitate on the filter. Both dishes and the filter are finally rinsed with hot water. The 
ammoniacal filtrate contains enough ammonium salts to prevent precipitation of magnesium in the first filtrate when the two come together.

If manganese peroxide has been deposited on the surface of the dish it is removed by hydrochloric acid and a drop or two of sulphurous acid, which mixture is then passed hot through the filter, the filtrate being caught in a small beaker. A reprecipitation by ammonia is then made, and the precipitate collected again on the filter and added to the main one, the filtrate passing into the flask containing the previous filtrate. If much manganese is present, of course a second precipitation by ammonia of the small precipitate may be required. In these cases there is no difficulty in getting all the manganese into the filtrate.

r. After precipitation by ammonia and ammonium persulphate.The filtrates are evaporated as in $\alpha$. The second is carried to dryness and the ammoniacal salts are removed by ignition. The residue is treated with a little hot hydrochloric acid and added to the first. A drop or two of sulphurous acid is then added if chromium is present, as will usually be shown by a yellowish color of the solution. The aluminum, chromium, and trace of iron are then precipitated by ammonia in slight excess and the digestion allowed to proceed on the bath till the slight precipitate has coagulated. This is collected on a small filter $(7 \mathrm{~cm}$.) and washed with hot water, the filtrate being collected as in $\alpha$ and $\beta$ if nickel or zinc are to be looked for, otherwise in a large platinum dish for treatment as in 10 (p. 118).

The precipitate is redissolved in hydrochloric acid, reprecipitated in small bulk of solution by ammonia, and finally added to the main precipitate obtained in c (p. 102).

e. IGNition OF the PRECIPITATE OF IRON, ALUMINUM, ETC., OXIDES.

The combined precipitates of alumina, etc., obtained in either $a, b$, $\mathrm{c}$, or $\mathrm{d}$, are ignited without preliminary drying in the crucible containing the residue from the silica (6. B. c, p. 95), unless considerable iron is present. In that case the main precipitate is dried and removed as far as possible from the paper, which is ignited separately to prevent partial reduction of a portion of the iron oxide. This is especially necessary when the precipitates were obtained without using macerated filter paper, in which case they are, after ignition, lumpy, and any magnetic oxide that may have been formed can not be wholly reoxidized by heating or by treatment with nitric acid (see 5. A. c. $\beta$, p. 71 ). They are also slow to yield to subsequent treatment. A precipitate formed in presence of thoroughly macerated paper, as recommended by Dittrich, on the other hand, yields on ignition a powder, and the fine state of division makes reoxidation of any 
reduced iron easy, besides wonderfully shortening the time required for subsequent re-solution.

Alumina in the quantities ordinarily found can not be fully dehydrated by the full heat of the Bunsen burner. It must be blasted for five or ten minutes. If iron is present in large amount this last operation must be conducted so as to insure access of air to the crucible (p. 89).

For ignitions of this kind, which involve no very prolonged heating, an electric furnace is admirably adapted by reason of its nonreducing atmosphere, provided the temperature is not high enough to cause by temperature alone reduction of ferric to magnetic oxide of iron.

The empty weight of the crucible, which may have changed since igniting the silica in it, is found after treatment of its contents as in the next section.

\section{f. RECOVERY OF SILICA AND POSSIBLE BARIUM IN THE ALUMINA PRECIPITATE.}

$\alpha$. Silica.-The weighed precipitate is transferred as far as possible to another crucible of 25 to 30 grams weight. The small adhering residue, chiefly that originally derived from the silica, is brought into solution by fusion with a small amount of sodium or potassium pyrosulphate. This fusion takes but a few minutes, and must not be continued beyond the time actually needed, otherwise it will be impossible, after cleaning and igniting, to get the correct weight the crucible possessed after the ignition of the alumina. ${ }^{a}$ The hot liquid is poured onto the main mass of precipitate in the other crucible, more pyrosulphate $^{b}$ is added (up to 7 grams in all ${ }^{c}$ ) and the fusion is renewed

$a$ It will be remembered (see page 94 , footnote $d$ ) that long blast or furnace ignition of the silica may have reduced the weight of the crucible by an appreciable amount, hence the need for getting its weight empty at this point. With crucibles of American manufacture the average loss up to this point due to blasting both silica and alumina in one and the same crucible may reach $0.8 \mathrm{mg}$. (mean of six tests by $\mathrm{R}$. C. Wells, ranging from 0.6 to $1.6 \mathrm{mg}$.).

$b A$ chief objection by many to the use of an alkali bisulphate for bringing this precipitate into solution is that so much care is needed during a fusion to prevent frothing and boiling over, which involves a very gradual application of the heat and consequent greater expenditure of time. The objection is sound as applied to the bisulphate, but is swept away if the latter is first converted into pyrosulphate, which is always its condition after the frothing has ceased. To effect this change it is only necessary to melt a large amount of the salt in a platinum dish and keep it in fusion till spattering ceases and white fumes begin to come off freely. If the liquid is then poured out into other dishes it cools in thin sheets or cakes, which can be readily broken up and bottled for future use. With such material the fusion is always tranquil, and the action on the alumina, etc., much more intense and speedy than with the ordinary bisulphate. If a good article of the latter is not to be had by purchase, it is only necessary to mix and melt together equivalent weights of sulphuric acid and pure normal sulphate until the water of the acid has been mostly expelled and the conversion to pyrosulphate accomplished.

Of late, the recommendation of J. Lawrence Smith (Am. Jour. Sci., 2 d ser., vol. 40, 1865, p. 248) to use the sodium instead of the potassium salt has been followed with satisfaction. This salt acts more quickly and forms a more soluble double salt with aluminum, but has the slight disadvantage that the progress of decomposition of the ignited mass is not so readily followed because of the greater tendency of the sodium salt to crust over.

A further objection is the action on the crucible of the fused pyrosulphate and the consequent necessity of removing the dissolved platinum. To obviate this and likewise to effect more speedy solution of the oxides E. Deussen (Zeitschr. anorg. Chemie., vol. 44, 1905, pp. 423-426), fuses the finely powdered oxides with acid potassium fluoride (about $1 \mathrm{gram}$ ). The mass melts at first over a low flame, then solidifles. 'Its 
and continued until the whole has been dissolved, which in the case of the potassium salt is always easy to be seen, even when the liguid appears dark red and opaque, by removing the crucible from the flame to a good light and allowing to cool. At one point in the cooling the liquid becomes transparent and allows the bottom of the crucible to be seen. This is less the case with the sodium salt.

When all is dissolved ${ }^{a}$ the melted mass is poured into a large, dry platinum dish, what adheres to the two crucibles is removed by diluted sulphuric acid, and the solution, with rinsings, is poured into the dish. Much more dilute sulphuric acid is added and the dish heated till the solid is dissolved. The solution is evaporated as far as can be done on the steam bath, then gradually heated higher till fumes of sulphuric acid come off copiously. During this heating the mass is apt to darken from reduction and separation of dissolved platinum. The acid should have been used in such quantity that after cooling the mass is pasty and not solid, for it then dissolves readily, on heating, in the water, which is now to be added, the sodium salt dissolving much more readily than the potassium salt. It is important that the amount of absolute acid used shall be approximately known, so that in the subsequent reduction of iron the volume of the solution can be so adjusted as to contain from 1.5 to 2.5 per cent by volume of acid.

The dish is placed on the bath and soon the silica can be seen in coagulated form at the bottom. It is collected on a small filter, well washed with hot water, burned, and weighed in a platinum crucible. Being rarely pure, it has to be corrected by a few drops of hydrofluoric and a single drop of sulphuric acid, and the crucible reweighed. The slight residue in the crucible is brought into solution by pyrosulphate and added to the main portion, for the treatment of which see $\mathrm{B}$, page 107, after filtering from barium if present (see $\beta$, p. 107).

The amount of silica thus recovered varies from 2 to 4 milligrams after two filtrations of the main silica, and is, of course, to be added to this.

Only in this way is the full amount of the silica in the alumina precipitate to be recovered. ${ }^{b}$ Formerly it was customary to regard that remaining undissolved when the pyrosulphate melt was taken up with water or dilute acid as the total amount. It is, however, but a small

solution is evaporated with sulphuric acid to expel most of the fluorine, then reduced with hydrogen sulphide or sulphurous acid and titrated for iron. The use of the fluoride is, however, incompatible with accurate work in silicate analysis, since the silica in the ignited oxides can not be determined. Moreover, the oxides must be finely powdered and consequently an aliquot part only can be taken for the fusion. The expulsion of fluorine by sulphuric acid must furthermore be thorough if titanium is to be determined in this solution.

$c$ The total weight of pyrosulphate used should be known approximately in order to correct later for its effect on the colorimetric determination of the titanium (p.134).

$a$ Should a small portion of the precipitate have escaped solution no particular harm is done. The further treatment is not thereby changed.

b For proof see Jour. Am. Chem. Soc., vol. 24, 1902, pp. 368-370; Chem. News, vol. 86, 1902, pp. 79, 89. 
portion of what the alumina held, the greater part having formed alkali silicate during the fusion and remaining in solution afterwards, unless set free and rendered insoluble by the treatment above given.

The silica thus recovered does not, however, represent quite all of that which originally escaped separation by evaporation with hydrochloric acid. A small part (roughly 1 to 2 milligrams for a single precipitation and a total of 1 centigram of silica in the experiments detailed elsewhere ${ }^{a}$ ) is not precipitated with the alumina and escapes recovery altogether. It is therefore apparent why a thorough separation of silica at the start is so urgently called for.

$\beta$. Barium.-Should by any chance the alumina precipitate have held barium sulphate, something which has not happened in my experience with rocks, unless ammonium persulphate was used as in c. $\alpha$, page 102, it will be found as a contaminant of the silica recovered after the pyrosulphate fusion. The weighed residue remaining after expulsion of this silica by hydrofluoric acid is dissolved (except the platinum it may have held) by a little pyrosulphate and the cooled melt redissolved in dilute sulphuric acid. Any barium will then be left in the form of sulphate, ${ }^{b}$ and can be separated by flotation from the heavier platinum.

\section{B. DETERMINATION OF IRON IN THE PRECIPITATE OF ALUMINA, ETC.}

\section{a. WITHOUT REGARD TO THE PRESENCE OF VANADIUM.}

$\alpha$. Reduction of the iron.-Into the cool filtrate obtained in $\mathrm{f}$. $\alpha, \beta$, pages 105-107, held in a beaker and containing from 1.5 to 2.5 per cent by volume of free acid, is introduced hydrogen sulphide for the reduction of the iron to the ferrous state and the precipitation of the platinum resulting from the pyrosulphate fusion. This last effect is rendered complete by causing the solution to boil c

\footnotetext{
a Jour. Am. Chem. Soc., vol. 24, 1902, p. 369; Chem. News, vol. 86, 1902, p. 90.

b Some years ago, in a series of analyses of rocks from the Leucite Fills, in Wyoming, there was obtained at this stige, when it was customary to dissolve the melt in cold water preliminary to precipitation of titanium by boiling the neutralized sulphuric solution in presence of sulphur dioxide, a white, more or less flocculent residue which amounted to 1 to 3 per cent of the rock and which was at first taken to be a mixture of tantalic and columbic acids. Eventually, it was found to consist apparently of nothing but $\mathrm{TiO}_{2}$ and $\mathrm{P}_{2} \mathrm{O}_{6}$, with perhaps a little $\mathrm{ZrO}_{2}$. By repeated fusion with acid potassium sulphate and leaching with cold water it could be gradually brought into solution. It was these rocks which furnished the most striking Instance of the peculiar milky sulphate residues mentioned on p. 96 as derived from the ignited silica.

Knop (Zeitschr. Kryst., yol. 10,1885, p. 73) seems to have obtained a similar mixture in analyzing minerals from the Kaiserstuhl in Baden, but its nature was not ascertained, though it was suspected to be, if not silica, columbiferous titanic acid.

$c$ It may be mentioned that the precipitation of platinum from a hot sulphate solution is far quicker and cleaner than from hydrochloric acid. Further, this platinum sulphide, when ignited in the crucible in which the bisulphate fusion was made, should weigh together with the crucible itself what the latter weighed before the main silica precipitate was ignited in it; in other words, the weight of the platinum recovered by hydrogen sulphide should equal the loss in weight of the crucible due to attack by the bisulphate. In somewhat rare instances this will not be so, but the weight will be greater, showing a gain in platinum which may amount to a milligram. Tests have shown that this is not due to retention of platlnum by the main $\mathrm{Al}_{2} \mathrm{O}_{3}$, etc., precipitate; hence, it must come from platinum mechanlcally loosened from the dish during the drying and powdering of the silica preparatory to its collection on the filter, or to some insoluble compound of vlatinum formed during evaporation and drying of the silica. It may also be in
} 
with continued passage of the gas, whereby the sulphur becomes coagulated and readily filterable. The gas current is not interrupted till the solution has cooled somewhat. This is then filtered ${ }^{a}$ warm into a half-liter flask, hydrogen sulphide is again introduced for a few minutes, with production of slight milkiness if any iron had become oxidized during filtration, the flask is then connected with a carbon-dioxide generator already in action for a time, and the solution brought to boiling to expel the hydrogen sulphide. The expulsion is aided by a rapid current of carbon dioxide, ${ }^{b}$ the end point being found by occasional testing of the escaping gas with lead-acetate paper. ${ }^{c}$ When this point is reached, the flame is removed and the flask allowed to cool without interrupting the gas current. The cooling is hastened by placing the flask in a basin of cold water.

$\beta$. Titration of the iron.-A burette with permanganate solution having been made ready, the gas tube is rinsed into the flask and the permanganate at once run into the latter without further dilution of

part or wholly due to contamination from reduction of platinum during evaporation of the filtrate from the basic-acetate separation. It will be remembered that from this filtrate a small amount of iron and alumina is recovered and added to the main precipitate. Hence it is always well in fine work to collect the sulphide and weigh the platinum in the original crucible, deducting any excess from the alumina, or else to get rid of the platinum by hydrogen sulphide before proceeding to the precipitation of alumina, etc. (See 7, p. 98.)

$a$ Filtration is not necessary if only precipitated sulphur and no sulphides are in suspension, since this is without reducing action on cold permanganate solution, as H. L. Wells and W. L. Mitchell and others before them have pointed out. The above authors used this method of reducing ferric iron in titanic iron ores. (Jour. Am. Chem. Soc., vol. 17, 1895, p. 878; Chem. News, vol. 73, 1896, p. 123.) The observation of A. Coppadoro (Gazz. chim. ital., vol. 31, pt. 2, 1901, p. 217) that the precipitate of sulphur caused by reducing ferric solutions with hydrogen sulphide carries some iron as sulphide, even in strongly acid solutions and after thorough washing, need not be regarded. He seems to have operated on very concentrated solutions as compared with those here in question.

$b$ The gas generated from every new lot of marble must be carefully tested for hydrogen sulphide. Even pure white marble is frequently not free from soluble sulphides-probably pyrrhotite, sphalerite, or galenaand the amount may be enough to appreciably affect the result of titration for iron. A good precaution to take is to wash the gas by passing it through a large $U$ tube containing glass beads and a solution of copper sulphate.

c The test is most delicate when the flask is provided with a two-hole stopper fitted with inlet and outlet tubes of glass. If the outside opening of the outlet tube is small, the concentration of the escaping steam and gas contributes to the sharpness of the test. Because of the possibility of the introduction of organic matter from the stopper by the action of the hot steam, it is safer to employ a cap of platinum foil with two holes, one for the gas entry tube, the other for its escape in a concentrated stream.

If the solution was free from milkiness at the start, the operation of expelling hydrogen sulphide need not take more than ten to twenty minutes; otherwise a longer time will be required to remove the last traces. The immediate effect of boiling on any moderate amount of suspended sulphur is to coagulate it and clear up the solution completely. Some of the sulphur volatilizes with the steam, but a further portion appears to be converted by it to hydrogen sulphide, so that a faint reaction for that gas can be obtained as long as any sulphur remains. This is probably the explanation for Washington's direction (Manual of the Chemical Analysis of Rocks, 1904, p. 112) to boil for about two hours, since by this method of reducing the iron, without coagulating the sulphur, the filtrates are always strongly milky. It is important to bear in mind that excess of hydrogen sulphide at this stage is not proof that all ferric iron has been reduced. Alkali sulphocyanate occasionally reveals the presence of unreduced iron. Due caution therefore renders it advisable to test a drop of the solution and to introduce more hydrogen sulphide if the result shows that it is needed. Experiments made under my direction at the Bureau of Standards by R. S. McBride show that perfect results are to be had only when the acid concentration is kept within very narrow limits. For the average run of rocks these may range from 1.5 to 2.5 per cent sulphuric acid by volume, but for iron ores a variation of even 0.5 per cent from 2 per cent produces a sensible error. Reduction is then incomplete with more than 2 per cent of absolute acid; with less than this amount too much permanganate is required. 
the solution, which is kept in rotatory agitation by an appropriate movement of one hand. The bulk of the solution will be from 100 to $200 \mathrm{~cm} .^{3}$ The drops of the titrating fluid that may fall upon the neck of the flask are washed down by aid of a wash bottle. The strength of the permanganate solution would best not much exceed 0.0025 gram of iron to the cubic centimeter, for the amounts of iron to be determined are moderate and often very small.

The results are strictly accurate, with the limitations set forth in the paragraph below, when care is taken with the reduction by hydrogen sulphide. The method is superior to that involving the use of zinc, since no foreign impurity affecting the result is introduced, and the ever present titanium is not affected, nor is vanadium reduced below the condition of $\mathrm{V}_{2} \mathrm{O}_{4}$, whereas nascent hydrogen converts it, in part at least, to $\mathrm{V}_{2} \mathrm{O}_{3} \cdot{ }^{a}$

r. Further treatment of the solution after titration.-The titrated solution is returned to a dish, which may be of porcelain, and evaporated to a bulk of much less than $100 \mathrm{~cm} .{ }^{3}$ if the rock is supposed to contain less than 1 per cent of titanium. For each additional 1 per cent of titanium the final bulk may be larger by $100 \mathrm{~cm} .^{3}$ The solution is transferred to a graduated flask with a capacity of 50,100 , or $200 \mathrm{crn}^{3}$ or more, as the case may be, enough hydrogen peroxide free from fluorides is added to fully peroxidize the titanium, the flask is filled to the mark and well shaken, and the titanium is determined colorimetrically (see p. 128).

\section{b. HAVING REgard to The pREsence of VANAdIUM.}

If vanadium is present the value found for iron will be in error by the amount of permanganate required to oxidize $\mathrm{V}_{2} \mathrm{O}_{4}$ to $\mathrm{V}_{2} \mathrm{O}_{5} .{ }^{b}$ The amount of the correction will differ according as titration of the iron is made after reduction by hydrogen sulphide or nascent hydrogen. If the former is used, as should always be the case, because of the ever present titanium, the vanadium is reduced by it to $\mathrm{V}_{2} \mathrm{O}_{4}$, which in its action on permanganate is equivalent to two molecules of $\mathrm{FeO}$, while the reduction goes further with hydrogen. After the first transitory pink blush throughout the liquid, the more slowly acting vanadium

a The statement in the foregoing paragraph regarding the superiority of the reduction by hydrogen sulphide is subject to correction if the method recently proposed by F. A. Gooch and H. D. Newton (Am. Jour. Sci., 4th ser., vol. 23, 1907, p. 365; Chem. News, vol. 96, 1907, p. 148; Zeitschr. anorg. Chemie, vol. 54, 1907, p. 213) reveals no defects after thorough trial. Those authors find that after reduction by zinc, titanium is completely oxidized to the guadrivalent state by either cupric oxide or bismuth oxide and that these oxides do not oxidize ferrous iron. They therefore reduce with zinc the solution of titanium and iron; to the flisk containing the cooled solution free from metallic zinc they add a little of one of the oxides (preferably that of bismuth), shake gently, filter quickly, and titrate with potassium permanganate. The results they furnish are very satisfactory.

$b$ This statement holds true also for the method of Gooch and Newton, referred to in the foregoing footnote, for the oxides of copper and bismyth do not, according to my tests, convert $\mathrm{V}_{2} \mathrm{O}_{4}$ to $\mathrm{V}_{2} \mathrm{O}_{5}$. According to a private communication from Newton bismuth trioxide converts vanadium that has been reduced to $\mathrm{V}_{2} \mathrm{O}_{2}$ by zinc, only to $\mathrm{V}_{2} \mathrm{O}_{3}$ if the solution is strongly acid (10 per cent $\mathrm{H}_{2} \mathrm{SO}_{4}$ by volume) and cool, but toward $\mathrm{V}_{3} \mathrm{O}_{4}$ if warm or less acid. 
may require the addition of a drop of two more of permanganate before a comparatively permanent coloration appears.

When the amount of vanadium in the rock is known, a correction can be applied on the assumption that practically all the vanadium is here collected, a point that needs further investigation. Various authors assert its precipitability-with alumina and iron by ammonia and ammonium acetate, though Carnot ${ }^{a}$ states that repeated precipitation by ammonia, ammonium carbonate, or ammonium sulphydrate separates it from iron. My experience with ores very rich in vanadium shows that precipitation along with iron and aluminum is only partial. Ridsdale ${ }^{b}$ has determined its precipitability with various metals and gives numerous figures which show an approximation to 90 per cent thus thrown down under the conditions prevailing in analysis of iron slags, the remainder passing into the filtrates and appearing in small part with the lime and to a greater extent with the magnesium phosphate. For all practical purposes it is probably safe to assume that the small amounts of vanadium met with in rocks are wholly in the alumina precipitate.

If the amount of vanadium in the rock is not known and great accuracy is necessary, caution requires the determination of the total iron to be made either in a separate portion or after reprecipitation from the above solution, as follows: Fuse with sodium carbonate, extract with water, bring the insoluble residue into sulphuric solution, reduce, and titrate as above directed. But unless a certain precaution is here observed an error greater than that which it is designed to avoid will be committed. Contrary to general belief, the aqueous extract from the sodium-carbonate fusion carries a small but appreciable fraction of a per cent of iron, as I have repeatedly found by actual test. This iron is thrown out with the alumina (and silica, if present) by the usual methods of neutralizing the alkaline solution, and can be brought to light when the precipitate thus formed is treated with a fixed caustic alkali, or again fused with sodium carbonate and leached with water, when it remains wholly or in part undissolved. Hence it is necessary to collect this iron and add it to the main portion before titration.

c. DETERMINATION OF THE TRUE VALUE FOR FERRIC IRON.

The total iron in the rock having in one way or another been found, it remains to deduct an amount equivalent to the ferrous oxide the rock contains and a further amount corresponding to the sulphides often present, in order to get what may pass for the true value for ferric iron. That this is often only an approximation appears from the difficulties due to the presence of vanadium and the generally indeterminable effect of sulphides on the ferrous-oxide determination. (See pp. 165-166.) 

ALUMINUM.

a. AFTER FIRST REMOVING IRON AS SULPHIDE.

Should it be desirable for any reason to effect an actual separation of aluminum, this may best be done, up to a certain point, after the pyrosulphate fusion (f, p. 105), by removal of the iron ${ }^{a}$ by ammonium sulphide in ammonium-tartrate solution, evaporation of the filtrate, ignition of the residue with sodium carbonate and nitrate, and extracttion with water, whereby titanium and zirconium are left on the filter as sodium salts, while chromium and vanadium are carried into the filtrate as chromate and vanadate along with aluminum and phosphorus. The further separation of the last two from the chromium and vanadium is outlined under "Phosphorus" (B, p. 145). This is as far as the separation can well be carried, and the $\mathrm{Al}_{2} \mathrm{O}_{3}$ must still be found by subtracting the $\mathrm{P}_{2} \mathrm{O}_{5}$ from the combined weights of the $\mathrm{Al}_{2} \mathrm{O}_{3}$ and $\mathrm{P}_{2} \mathrm{O}_{5}$. The possibility of loss of some $\mathrm{P}_{2} \mathrm{O}_{5}$ by volatilization ${ }^{b}$ during the bisulphate fusion must be borne in mind here, for if it takes place the final weight of $\mathrm{Al}_{2} \mathrm{O}_{3}+\mathrm{P}_{2} \mathrm{O}_{5}$ will not contain all the $\mathrm{P}_{2} \mathrm{O}_{5}$.

Some writers have recommended dissolving the ignited alumina, iron oxide, etc., in hydrochloric acid, but when the precipitate has been heated over the blast, as it should be, this is very ineffective.

\section{b. By EXTRACTION WITH A FIXED CAUSTIC ALKaLI.}

A favorite practice in some countries of Europe has been to fuse the ignited precipitate containing $\mathrm{Al}_{2} \mathrm{O}_{3}, \mathrm{Fe}_{2} \mathrm{O}_{3}, \mathrm{TiO}_{2}, \mathrm{P}_{2} \mathrm{O}_{5}$, etc. -or that of the $\mathrm{Al}_{2} \mathrm{O}_{3}, \mathrm{TiO}_{2}, \mathrm{P}_{2} \mathrm{O}_{5}$, etc., after separation of iron by ammonium sulphide in tartrate solution - with sodium hydroxide in a silver crucible, or to boil the freshly precipitated mixture with a solution of the alkali, on the assumption that the oxide of titanium is hereby rendered wholly insoluble and thus separated from the alumina. This, however, is in part an error long since pointed out by F. A. Gooch, ${ }^{c}$ who showed that pure titanic oxide is markedly soluble under both conditions of treatment. Experiments made by myself to test the extent of this error brought out the following interesting results:

When 0.045 gram of titanic oxide was fused by itself with sodium hydroxide, the clear aqueous extract of the fusion held $0.0031 \mathrm{TiO}_{2}$, or about 7 per cent, determined colorimetrically. When freshly precipitated and boiled with the alkali, the solubility was less. When

a This being first reduced to the ferrous condition by hydrogen sulphide in acid solution in order to obviate the possibility of precipitating some titanium, which otherwise is likely to happen. (Cathrein, Zeitschr. Kryst., rol. 6, 1882, p. 246, and vol. 7, 1883, p. 250.

$b$ H. R.ose speaks of such loss when volatilizing sulphuric acid in presence of phosphoric acid (Handb. f. quant. Anal., Finkener ed., vol. 2, 1871, p. 575, and elsewhere), and the fact of such loss to a very marked extent has been confirmed in this laboratory.

c Proc. Am. Acad. Arts Sci., vol. 12, 1885, p. 436; Bull. U. S. Geol. Survey No. 27, 1886, pp. 16, 17. 
fused with sodium carbonate, but an infinitesimal trace was dissolved, which required strong concentration for its detection. When mixed with a large excess of alumina and fused with the caustic alkali, the solubility was still very marked, though less than when alumina was absent. With a large excess of ferric oxide, with or without alumina, no titanium could be detected in the unconcentrated filtrate.

It thus appears that fusion with caustic alkali after first removing iron involves an error in the gravimetric determination of both aluminum and titanium which does not appear if the iron has not been removed.

\section{c. METHODS OF DITTRICH.}

Evidently with a view to eliminating some sources of error inherent in the methods outlined in $a$ and $b$ above, Dittrich evolved a procedure $^{a}$ which is a combination of both and includes methods of separating and determining titanium and zirconium. In outline it is as follows, the starting point being the precipitate produced by ammonia and hydrogen peroxide, and containing several or all of the oxides: $\mathrm{Fe}_{2} \mathrm{O}_{3}, \mathrm{Al}_{2} \mathrm{O}_{3}, \mathrm{Cr}_{2} \mathrm{O}_{3}, \mathrm{TiO}_{2}, \mathrm{ZrO}_{2}, \mathrm{Mn}_{3} \mathrm{O}_{4}, \mathrm{P}_{2} \mathrm{O}_{5}$, and $\mathrm{SiO}_{2}$.

After weighing, this precipitate is fused in a silver crucible with sodium hydroxide, whereby $\mathrm{Al}_{2} \mathrm{O}_{3}$ and $\mathrm{P}_{2} \mathrm{O}_{5}$ are brought into solution. If manganese has been in part dissolved as manganate, it is rendered insoluble by digesting the aqueous solution of the melt with hydrogen peroxide.

The filtrate is evaporated with hydrochloric acid to separate $\mathrm{SiO}_{2}$, and the $\mathrm{Al}_{2} \mathrm{O}_{3}$ and $\mathrm{P}_{2} \mathrm{O}_{5}$ are precipitated by ammonia and weighed together. Thèn the $\mathrm{P}_{2} \mathrm{O}_{5}$ in the mixture is determined, if present, thus giving the $\mathrm{Al}_{2} \mathrm{O}_{3}$ by difference. Chromium is not considered by Dittrich in this scheme, but its determination colorimetrically would present no difficulty (17, p. 147).

The treatment of the oxides insoluble in sodium hydroxide is described under $b$, page 136 , according to the more recent directions of Dittrich and S: Freund. ${ }^{b}$

\section{d. DIRECT PRECIPITATION OF ALUMINUM BY PHENYLHYDRAZINE.}

A recent and promising method for the "direct determination of alumina in presence of iron, manganese, calcium, and magnesium" is that of Hess and Campbell, ${ }^{c}$ but, as with the methods just considered, it involves finally weighing aluminum, phosphorus, and titanium together. Precipitation is made by phenylhydrazine, after first neutralizing the (preferably chloride) solution by ammonia and reducing iron by a saturated solution of ammonium bisulphite. 
Phenylhydrazine "precipitates aluminum from its solutions quantitatively as the hydroxide without a trace of the precipitate being redissolved in excess of the precipitant."

Dr. E. T. Allen has investigated the method in this laboratory ${ }^{a}$ and confirmed the above authors' statements in the main, finding, however, that two precipitations are needed to free the precipitate entirely from iron. "The method is excellently adapted to the separation of very small quantities of aluminum, such as a milligram or even less, from a large excess of iron, a point of considerable practical importance."

Although such conditions will not be met with in ordinary rock analysis, it will not be out of place here to give the treatment as slightly modified by Doctor Allen:

The volume of the solution may vary, according to the quantity of alumina to be precipitated, from 100 to $200 \mathrm{~cm}^{3}$. It should be heated and reduced by adding saturated ammonium bisulphite. From 5 to 20 drops, according to the quantity of iron, rnay be used. If the solution turns deep red (ferric sulphite), it is not acid enough, and a few drops of hydrochloric acid should be added, for the sulphite itself does not reduce ferric salts, at least not with rapidity. Now quickly bring to neutrality with ammonia, and then add several drops of dilute hydrochloric acid. If this last operation is done too slowly the oxygen of the air helps to form a little ferric hydroxide which does not always readily dissolve in the dilute acid. Finally, add from 1 to 3 $\mathrm{cm} .^{3}$ of phenylhydrazine, $b$ according to the weight of the alumina to be precipitated. If too little has been used, a few drops added to the filtrate will disclose the mistake. Stir until the precipitate has become sufficiently flaky and allow to settle. The supernatant liquid will now be plainly acid to litmus. One need not be disturbed if the precipitate has a brownish color, for it is not due to ferric hydroxide, but to the coloring matter contained by all phenylhydrazine which has not been freshly distilled. When the determinations are allowed to stand too long the air increases this oxidation product, and a brown. insoluble scum forms on the surface of the liquid and on the sides of the vessel, which is rather troublesome to the analyst. Fortunately equilibrium appears to be established in a short time. The vessels need not stand more than an hour, at any rate. The precipitate is washed by a solution of phenylhydrazine sulphite made by adding cold saturated sulphurous acid to a little phenylhydrazine until the crystalline sulphite first formed dissolves in the excess. The solution has an acid reaction. Five to $10 \mathrm{~cm} .{ }^{3}$ of this are used in $100 \mathrm{~cm} .^{3}$ of hot water.

\section{MANGANESE, NICKEL, COBALT, COPPER, AND ZINC.}

\section{A. DIFFICULTIES IN THE WAY OF A CORRECT GRAVIMETRIC DETERMINATION OF MANGANESE.}

The gravimetric determination of manganese in small amounts seems to be more of a stumbling block to the average chemist than that of almost any other of the frequently occurring elements met with in mineral analysis. This is due almost always to incomplete

a Jour. Am. Chem. Soc., vol. 25, 1903, p. 421.

$b$ The reagent should, of course, be free from inorganic impurities which could disturb the results. The author [Allen] found one sample which after persistently giving high results was proved to contain tin, which had probably been used in its preparation. 
prior separation of elements which later suffer coprecipitation with the manganese. The error is therefore generally a plus one, and often amounts to many times the weight of the manganese actually present. The importance of thorough separation of the constituents treated of in the foregoing pages is therefore manifest, particularly since to the inexperienced analyst very small amounts of manganese precipitated in the form of a higher hydroxide may easily mask a good deal of alumina or other colorless precipitates. ${ }^{a}$

If the directions already given have been carefully followed, however, there will be little chance of error due to foreign contamination, either by alumina or magnesia, a sufficiency of ammonium salts being a guaranty against the latter. Regard must be had, however, to the rather remote possibility of the presence of rare earths which were not thrown out by the basic-acetate precipitation (see second footnote, p. 100), for they will appear at this stage.

\section{B. PRECIPITATION OF THE GROUP AND SEPARATION OF ITS CONSTITUENTS.}

a. THE AMMONIUM-SULPHIDE METHOD.

$\alpha$. Its advantages and disadvantages.-On the other hand, the usual methods of separation of manganese from the alkaline earths and magnesia by bromine or ammonium sulphide are imperfect, in part by reason of incompleteness, in part because of coprecipitation of small amounts of these metals. The former error, though absolutely slight, is in rock analysis probably of more consequence than the

$a$ It is for these reasons that Washington deems it better for the novice in rock analysis to make no attempt to determine the manganese, but to allow the error from this neglect to distribute itself over the alumina, lime, and magnesia. The relative order of this distribution has been determined by George Steiger in the Survey laboratory recently, while analyzing a series of carbonate rocks, some of them highly siliceous. The results are instructive. Double precipitations were the rule, and iron and aluminum were thrown out by ammonia.

Tests showing distribution of manganese in gravimetric separations.

\begin{tabular}{|c|c|c|c|c|c|c|c|}
\hline \multirow{3}{*}{ No. } & \multicolumn{3}{|c|}{ Partial composition of rock. } & \multicolumn{4}{|c|}{ MnO by colorimetry. } \\
\hline & \multirow[b]{2}{*}{$\begin{array}{l}\mathrm{Al}_{2} \mathrm{O}_{3} \\
\mathrm{Fe}_{2} \mathrm{O}_{3}\end{array}$} & \multirow[b]{2}{*}{$\mathrm{CaO}$} & \multirow[b]{2}{*}{$\mathrm{MgO}$. } & \multirow[b]{2}{*}{ Total. } & \multicolumn{3}{|c|}{ With- } \\
\hline & & & & & $\begin{array}{c}\mathrm{Al}_{2} \mathrm{O}_{8} \\
\mathrm{Fe}_{2} \mathrm{O}_{3}(\mathrm{by} \\
\text { difference). }\end{array}$ & $\mathrm{CaO}$. & $\mathrm{Mg}_{2} \mathrm{P}_{2} \mathrm{O}_{7}$ \\
\hline $\begin{array}{l}957 \ldots \ldots \ldots \\
973 \ldots \ldots \ldots \\
974 \ldots \ldots \\
975 \ldots \ldots \ldots \\
1126 \ldots \ldots \\
1128 \ldots \ldots \ldots \\
1130 \ldots \ldots \\
1131 \ldots \ldots \ldots\end{array}$ & $\begin{array}{r}2.03 \\
9.35 \\
4.80 \\
12.71 \\
.58 \\
.98 \\
3.49 \\
1.00\end{array}$ & $\begin{array}{r}10.60 \\
11.84 \\
50.51 \\
11.98 \\
30.54 \\
29.69 \\
3.99 \\
28.04\end{array}$ & $\begin{array}{r}6.30 \\
2.81 \\
1.04 \\
4.30 \\
20.41 \\
19.07 \\
.92 \\
19.11\end{array}$ & $\begin{array}{l}0.193 \\
.311 \\
.700 \\
.442 \\
.281 \\
.245 \\
.016 \\
.574\end{array}$ & $\begin{array}{r}0.085 \\
.036 \\
.301 \\
.088 \\
.030 \\
.019 \\
.016 \\
.032\end{array}$ & $\begin{array}{r}0.011 \\
.023 \\
.087 \\
.016 \\
.030 \\
.055 \\
\text { None. } \\
.101\end{array}$ & $\begin{array}{r}0.097 \\
.252 \\
.312 \\
.338 \\
.221 \\
.171 \\
\text { None. } \\
.441\end{array}$ \\
\hline
\end{tabular}

The results in 974 and 1130 particularly show how the manganese once precipitated tends to remain with the alumina, presumably by reason of its having become peroxidized. 
latter. In regard to completeness of precipitation bromine offers no advantage over ammonium sulphide, and the latter has the advantage that by a single operation nickel, cobalt, copper, and zinc, if present, are likewise separated from the earth metals and alkalies. There need be no fear of overlooking nickel or copper, for under the conditions of the precipitation they are not retained in solution. It is for this reason chiefly that the ammonium-sulphide method is to be preferred. Most of the small amount of manganese that escapes precipitation is weighed later with the magnesium as pyrophosphate and can be readily corrected for by the colorimetric method described on page 116.

The precipitation of manganese in alkaline solution by hydrogen peroxide, as proposed by P. Jannasch and E. v. Cloedt, ${ }^{a}$ a method which appeared to be simple and accurate, besides affording a separation from zinc, has been shown by C. Friedheim and E. Brühl ${ }^{b}$ to be valueless.

$\beta$. Precipitation by ammonium sulphide and separation of manganese and zinc from nickel, cobalt, and copper.-Two or three cm. ${ }^{3}$ of ammonia are added to the flask containing manganese, the earth metals, etc. (8. A. d. $\alpha$ and $\beta$, p. 103), and hydrogen sulphide gas is introduced to saturation, whereby manganese, nickel, cobalt, copper, zinc, and a small part of the platinum from the dish are precipitated. A second like amount of ammonia is now added. The flask, filled to the neck and corked, is set aside for at least twelve hours, and preferably twenty-four, or even longer. The precipitate, collected and washed on a small filter with water containing ammonium chloride and sulphide, is extracted by hydrogen-sulphide water acidified with one-fifth its volume of hydrochloric acid (sp. gr. 1. 11), manganese and zinc, if present, going into solution.

(For treatment of the ammonium-sulphide filtrate see 10, p. 118.)

b. MANGANESE AND ZINC.

The hydrochloric-acid filtrate is evaporated to dryness, ammonium salts are destroyed by evaporation with a few drops of sodium-carbonate solution, hydrochloric acid and a drop of sulphurous acid are added to decompose excess of carbonate and to dissolve precipitated manganese, and the latter is reprecipitated at boiling heat by sodium carbonate after evaporation of the hydrochloric acid. If zinc is present, it can be separated from the manganese after weighing. For the small quantities of manganese usually found the sodiumcarbonate method of precipitation is to be preferred to that by bromine or sodium phosphate, as equally accurate and a time saver.

The manganese is weighed as $\mathrm{Mn}_{3} \mathrm{O}_{4}$ and calculated to $\mathrm{MnO}$, or, 
if present in some quantity, it may preferably be weighed as the sulphate, ${ }^{a}$ or, finally, it may be determined colorimetrically with much greater certainty, as in $\mathrm{C}$, below, when the amount is within the usual limits for rocks. In the last case, in the absence of zinc, the hydrochloric-acid solution of the sulphide may be used for the colorimetric test, after first evaporating off the hydrochloric acid with nitric or sulphuric acid.

\section{NICKEL, COBALI, COPPER.}

The paper containing nickel, cobalt, and copper is incinerated in porcelain, dissolved in a few drops of aqua regia, and evaporated with hydrochloric acid; the copper and platinum are thrown out warm by hydrogen sulphide, and in the filtrate made ammoniacal nickel and cobalt are thrown down by hydrogen sulphide. This liquid is then rendered faintly acid by acetic acid and allowed to stand. The sulphide of nickel after filtering is simply burned and weighed as oxide-its weight being always very small, hardly ever over 0.3 or 0.4 milligram-and is then tested for cobalt in the borax bead. The color of the last drop or two of the hydrochloric-acid solution, after decomposing the aqua regia, is a good indication of the presence or absence of nickel and cobalt. It is always well at this point to separate by ammonia any traces of iron that may be present, then to acidify and treat with hydrogen sulphide as above.

It is somewhat unsafe to consider traces of copper found at this stage to belong to the rock if the evaporations have been conducted, as is usually the case, on a copper water or steam bath, or if water has been used which has been boiled in a copper kettle, even if tinned inside. Therefore, and because of its contamination by a little platinum, it is better to determine copper in a separate portion if its presence is indicated with certainty. (See 7, p. 98.) This warning is particularly to be heeded if copper or brass sieves were used in preparing the sample.

\section{COLORIMETRIC DETERMINATION OF MANGANESE.}

a. PRELIMINARY TREATMENT.

As has been shown above (p. 113), the gravimetric determination of manganese is subject to grave error, even when great care is used. This is due to a variety of reasons, such as incompleteness of separation from aluminum and iron, incomplete precipitation by ammonium sulphide, and contamination by other bodies. Because of the small amounts in question these errors may be relatively enormous, and they can not be depended on to balance each other. Therefore, it

\footnotetext{
a Volhard, J., Ann. Chem., vol. 198, 1879, p. 329. Gooch, F. A., and Austin, Martha, Am. Jour. Sci., 4th ser., vol. 5, 1898, p. 209; Zeitschr. anorg. Chemie, vol. 17, 1898, p. 264.
} 
would be an advance if the total manganese could be determined in a separate portion of the sample, and in the main portion only that part which is weighed with the magnesium pyrophosphate, and this only as a correction to the magnesia. This course is readily feasible with carbonate rocks, which can usually be brought into a fit state in a few minutes without a fusion or the separation of silica. With silicate rocks more labor is involved, but the determination itself is very accurately performed by colorimetry, as in the case of carbonates.

The preliminary treatment consists in decomposing a gram or half a gram of the rock powder in a small platinum dish or capacious crucible (placed in the radiator shown in fig. 2, p. 31) with hydrofluoric and sulphuric acids till all is disintegrated. The hydrofluoric acid is then to be driven off by repeated evaporations with small portions of sulphuric acid; after which either sulphuric or nitric acid free from chlorine is added, together with water, and as much of the residue is brought into solution as possible. What remains insoluble is usually barium and calcium sulphates. This is filtered off on a small filter, the filtrate is caught in a small beaker or flask, and the manganese determined as in the following paragraphs.

\section{b. COLORIMETRIC DETERMINATION OF MANGANESE BY AMMONIUM PERSULPHATE. $a$}

This method has the advantage over that by lead peroxide in that, unless chlorides happen to be in the solution, no filtration is necessary. Two solutions are needed, one of silver nitrate containing 2 grams of the salt to the liter, and one of manganous sulphate or nitrate containing the equivalent of $2 \mathrm{mg}$. of $\mathrm{MnO}$ in $10 \mathrm{~cm}^{3}$. This last can be conveniently made by acidifying a standard permanganate solution, reducing it by sulphurous acid, and diluting appropriately. A solution of permanganate can be used directly as a color standard when very small quantities of manganese are in question. Ammonium persulphate in the solid form is also needed. The commercial article needs no purification ordinarily in this case.

The rock solution must be strongly acid with nitric or sulphuric acid and considerably less than $100 \mathrm{~cm}^{3}$ in bulk unless the manganese exceeds 1 milligram in weight. To it is added $10 \mathrm{~cm}^{3}$ of the silver solution for every milligram of metallic manganese, and it is transferred to a graduated flask of size suited to the solution. Should it show a turbidity from precipitated silver chloride, the liquid must be agitated and then filtered into the flask. There is now added about 1 gram of solid ammonium persulphate and the flask is placed on the steam bath or hot plate. Very soon the pink color of permanganic acid begins to appear and rapidly increases to a maximum. The flask may be removed soon after the color begins to show and placed in cold water when it is fully developed. If the depth of color is very

a Walters, H. E., Chem. News, vol. 84, 1901, p. 239 ; Proc. Eng. Soc. West. Pa., vol. 17, 1901, p. 257. 
great the solution must be poured into a larger flask. The flask is filled with water to the mark and the contents thoroughly mixed.

According to the depth of color there is now placed in another flask of $100 \mathrm{~cm} .{ }^{3}$ or less volume $10 \mathrm{~cm} .{ }^{3}$, or some multiple of that amount, of the manganese solution, so that when both solutions are oxidized and the flasks filled the color in the standard shall be more intense than in the test solution. This solution is oxidized by the aid of silver salt and persulphate in the same manner as the other, cooled, filled to the mark, shaken, and poured into a burette. Ten $\mathrm{cm}^{3}{ }^{3}$ is drawn off into one of the comparator cylinders shown in fig. 5 (p. 34), and diluted with water from a burette till the color equals that of the properly diluted and mixed test solution, a part or all of which has been poured into the companion cylinder. The observations are made exactly as given on page 34 . If the form of colorimeter depicted in figs. $6-7$ is used, the dilution of the standard solution is, of course, unnecessary. Nessler cylinder's can be used instead of either of the colorimeters described. The results are very exact.

Should, perchance, a brown precipitate form during the oxidation of the standard (due to deficiency of silver salt), it will sometimes be necessary to start with a fresh portion, but often the error can be remedied by immediate addition of more silver salt and continued heating. If this treatment fails with the test solution, the precipitate must be brought into solution by a little sulphurous acid, more silver salt added, and the oxidation repeated with persulphate.

\section{CALCIUM AND STRONTIUM (BARIUM).}

\section{A. SEPARATION FROM MAGNESIUM.}

a. PRECIPITATION AND IGNITION OF CALCIUM AND STRONTIUM OXALATES TOGETHER.

The platinum derived from the dish in the silica evaporation, except for the small portion precipitated with the manganese sulphide, is now wholly in the filtrate from the latter (9. B. a. $\beta$, pp. 114-115). Its separation at this or any other stage is quite unnecessary; nor is the removal of ammonium chloride usually demanded, since there is no undue amount present in most cases, the first precipitation of alumina, etc., having been by ammonium or sodium acetate. ${ }^{a}$ Therefore, without destroying ammonium sulphide the calcium and strontium are thrown out by ammonium oxalate at boiling heat, the precipitate, often darkened by deposited platinum sulphide, is ignited and redissolved in hydrochloric acid, boiled with ammonia to throw out traces of alumina sometimes present, filtered and reprecipitated as before, but in a small bulk of solution. It is weighed as oxide, transferred to a small flask of $20 \mathrm{~cm} .^{3}$ capacity, dissolved in nitric acid, evaporated to dryness at $150^{\circ}$

a If two or three precipitations by ammonia alone are depended on, the second and third filtrates are evaporated rapidly to dryness and the ammonium salts removed by ignition. 
to $160^{\circ}$, and the separation of strontium from calcium effected by ether-alcohol ${ }^{a}$ as described below.

'The weight of strontia found deducted from that of the two oxides gives that of the lime. For treatment of the filtrates see "Magnesium" (11, p. 123).

b. NOTES ON DOUBLE PRECIPITATION, WASHING, AND IGNITION OF THE OXALATES.

It may be said with regard to the separation of calcium from magnesium that two precipitations by ammonium oxalate are essential to the attainment of correct results, not only for the complete removal of magnesium but of sodium as well, the retention of compounds of the latter element by calcium oxalate being now generally known. This subject has been thoroughly studied by T. W. Richards, C. T. McCaffrey, and $\mathrm{H}$. Bisbee. ${ }^{b}$ They also call attention to the danger of too long washing with hot water because of the very appreciable solubility of calcium oxalate. This is a point that needs greater attention than it ordinarily receives from analysts.

The ignition is started with the precipitate moist. After charring and burning off the paper the crucible is to be covered and blasted with an inclined flame. It is a very mistaken idea that with a really good blast it takes long to reach constant weight. Five minutes is ordinarily ample.for any amount of lime that is likely to be met with. For ordinary amounts the heat of a large and good Bunsen burner is in reality sufficient, since I was able by it to reduce 3 grams of calcium carbonate in fifty minutes to the oxide, or so nearly so that the blast afterwards caused a further loss of only $1 \mathrm{mg}$. This, of course, was with the crucible covered. The lime should not be left over half an hour in the desiccator before weighing, for it gains weight appreciably if left long.

\section{B. SEPARATION OF STRONTIUM (BARIUM) FROM CALCIUM.}

a. BY ETHER-ALCOHOL.

The thoroughly dried nitrates obtained in A. a are treated with as little (rarely over $2 \mathrm{~cm}^{3}$ ) of a mixture in equal parts of absolute alcohol and ether as may be needed to dissolve the calcium salt, solution being hastened by occasional gentle agitation. After standing overnight in the corked flask the insoluble matter is collected on the smallest possible filter and washed with more of the above mixture of alcohol and ether. After drying, a few cubic centimeters of hot water are passed through the filter, on which may remain a few tenths of a milligram of residue, which does not usually contain any lime or other

a See R. Fresenius, Zeitschr. anal. Chemie, vol. 32, 1893, pp. 189, 312, for the latest improvements in this method, in their application to considerable quantities.

o Proc. Am. Acad. Arts Sci., vol. 36, 1901, p. 375 ; Zeitschr. anorg. Chemie, vol. 28, 1901, p. 71. 
alkaline earth and whose weight is therefore to be deducted from that of the lime, unless it can be shown that it is derived from the glass of the little flask in which the nitrates of calcium and strontium were evaporated. To the solution of strontium nitrate in a small beaker a few drops of sulphuric acid and then its volume of alcohol are added, whereby the strontium is precipitated as sulphate, in which form, after twelve hours, it is weighed and then tested spectroscopically as to freedom from calcium and barium.

b. BY AMYL ALCOHOL.

Because of the slight solubility of strontium nitrate in amyl alcohol the method of Browning ${ }^{a}$ does not appear to be adapted to the separation from calcium of the small amounts of strontium met with in rocks, though with barium the case is different, since its nitrate, according to Browning, is insoluble in absolute amyl alcohol.

\section{BEHAVIOR OF BARIUM.}

Barium, after two ammonium-oxalate precipitations, will never be found with the ignited calcium and strontium in more than spectroscopic traces, unless originally present in excess of $3 \mathrm{or} 4 \mathrm{mg}$., and very often only when in considerable excess. ${ }^{b}$ If present with them, however, it will be separated with the strontium by ether-alcohol or amyl alcohol, and these two must then be treated by the ammoniumchromate method, given below, in order to arrive at the strontium. The barium is best estimated in a separate portion. (See "Barium," 13, p. 138.

\section{SEPARATION OF BARIUM FROM STRONTIUM.}

Profiting by the experience of $R$. Fresenius and others, A. Skrabal and L. Neustadl ${ }^{\circ}$ have shown in what manner alone a very nearly correct separation of barium can be made from calcium and strontium. The following solutions are requisite:

Ammonium bichromate, 100 grams to the liter.

Ammonium acetate, $a, 300$ grams, neutralized by ammonia, to the liter.

Ammonium acetate, $b, 20 \mathrm{~cm}^{3}$ of $a$ diluted to 1 liter.

The reaction of the acetate solutions should be alkaline rather than acid.

The method for the amounts used by the authors $(0.135$ gram $\mathrm{BaO}$ as the chloride and the same of strontium) is as follows:

To the neutral or weakly acid solution is added ammonium acetate $\left(10 \mathrm{~cm} \cdot{ }^{3}\right.$ of solution $a$ ) in excess. The liquid is brought to boiling and

a Am. Jour. Sci., 3d ser., vol. 43, 1892, pp. 50, 314.

$b$ Hillebrand, W. F., Jour. Am. Chem. Soc., vol. 16, 1894, p. 83; Chem. News, vol. 69, 1894, p. 147.

c Zeitschr. anal. Chemie, vol. 44, 1906, p. 742. 
while swirled about $5 \mathrm{~cm}^{3}$ of the bichromate solution is added. After settling and cooling the ${ }^{\ell}$ clear liquid is decanted through a filter and the precipitate washed by decantation with ammonium acetate (solution $b$ ) till the filtrate is no longer perceptibly colored $\left(100 \mathrm{~cm} .{ }^{3}\right.$ of wash solution). The beaker is placed under the funnel, the precipitate on the paper dissolved by warm dilute nitric acid, and the paper washed. More acid is then added to dissolve the rest of the precipitate, followed by ammonia till the precipitate forming again no longer redissolves. Ammonium acetate $\left(10 \mathrm{~cm}^{3}\right.$ of solution $\left.a\right)$ is now poured in, the liquid brought to boiling while kept in swirling motion, allowed to cool slowly, and the precipitate is washed by decantation with solution $b$ of the ammonium acetate. The barium chromate is dried and weighed as such after ignition, the filter being burned separately.

The strontium may be thrown down from the combined filtrates by ammonia and ammonium carbonate, after concentration in presence of a little nitric acid, and weighed as carbonate; or the carbonate may be redissolved, precipitated by sulphuric acid and alcohol, and weighed as sulphate.

It is probable that for the small amounts encountered in rocks a single precipitation of the barium by bichromate will suffice, but this is not so when any considerable quantities are to be separated.

\section{E. OTHER METHODS OF DETERMINING THE WEIGHT OF OALCIUM.}

GENERAL STATEMENT.

Additional methods more or less in vogue for determining the calcium after precipitation as oxalate are the volumetric method by potassium permanganate and the conversion of the oxalate into carbonate, sulphate, or fluoride. None of these is applicable in rock analysis if it is desired to determine the strontium, except the second, but any one of them will apparently afford good results if no account is to be taken of that element. The methods have, however, not been tested in this laboratory, though the several gravimetric methods have been compared by O. Brunck, ${ }^{a}$ who finds that duplicates by any one of them show better agreement than by the oxide method. Brief descriptions of them are therefore given below, as well as of the volumetric method.

It is to be said, however, regarding Brunck's determination of calcium as the oxide that no such variations as those reported by him are ever obtained in the Survey laboratory. Our duplicates by that method agree as well as his by the other methods. His high and varying results by the oxide method are probably to be attributed to 
the employment of a vertical instead of an inclined flame. The complete conversion of the oxide to sulphate by treatment with either sulphuric or hydrofluoric acid is, in my opinion, based on experience with the conversion of lead chloride to sulphate, by no means easily brought about. There will always be a tendency, where an insoluble compound is at once produced by action of one of the acids in question, for some of the original compound to be protected from conversion to the salt, even after two or more evaporations with fresh portions of acid.

It is further doubtful if the loss in weight of the crucible during blast ignition of the lime is really as. great as he thinks. It is well known that barium oxide attacks platinum strongly when heated in contact with it, and it is certain that lime does so to a less but appreciable extent. That it does can readily be demonstrated by dissolving in hydrochloric acid lime that has been blasted in platinum for half an hour and precipitating and weighing the dissolved platinum. The amount recovered will nearly and sometimes quite equal the loss in weight of the crucible itself.

\section{a. VOLUMETRIC METHOD.}

The washed oxalate is rinsed into a beaker and the filter is extracted with hot dilute sulphuric acid, the filtrate being caught in the beaker. It is not permissible to omit this extraction, for simple rinsing with water will not extract all of the oxalate held in the pores of the paper. More acid is added if necessary, and the contents of the beaker are digested at a moderate heat till decomposition can be considered complete. The oxalic acid is then titrated warm at about $70^{\circ}$ with permanganate, which has been standardized best against pure Iceland spar dissolved in hydrochloric acid and precipitated as the oxalate.

\section{b. BY WEIGHING AS THE CARBONATE.}

The oxalate is gently ignited to convert it to carbonate and is then digested with a few drops of a solution of ammonium carbonate. The liquid is evaporated cautiously and the residue gently ignited to just visible dull redness and weighed. The treatment with ammonium carbonate, etc., is repeated till constant weight is reached.

\section{c. BY WEIGHING AS THE SULPHATE.}

The strongly ignited oxalate is cautiously slacked with water and a slight excess of sulphuric acid is added. The liquid is concentrated by evaporation and the excess of acid removed in the radiator (fig. 2, p. 31). The dry sulphate is then moderately ignited (to dull redness for a short time) and weighed, the treatment with acid, etc., being repeated to constant weight. 
d. BY WEIGHING AS THE FLUORIDE.

The strongly ignited oxide is slacked with water and covered with hydrofluoric acid, the excess of which is removed on the bath and the residue ignited for a few moments and weighed. The treatment with acid, etc., is to be repeated till the weight becomes constant. According to Brunck this last method is simpler than the carbonate or sulphate method, and in the hands of the inexperienced leads to better results, though he regards the sulphate method as the best in experienced hands.

\section{MAGNESIUM.}

\section{A. PRECipitation.}

a. CONDITIONS NECESSARY FOR THE PRODUCTION OF A NORMAL PRECIPITATE.

There are few analytical procedures regarding which so much uncertainty has existed and still exists as that by which magnesium is precipitated as the ammonio-phosphate, notwithstanding the enormous mass of detailed work that has been done on the subject. The work of H. Neubauer, ${ }^{a}$ confirmed in part and supplemented by F. A. Gooch and. Martha Austin, ${ }^{b}$ seemed to clear the ground fairly well. Neubauer found that precipitation is complete even in the presence of large quantities of salts of ammonium, including the oxalate, but that the composition of the precipitate is largely affected by ammonium salts and also by the way in which the precipitation is made. Gooch and Austin show that the large amount of ammonia of 0.96 specific gravity (one-third the original volume of the solution) usually prescribed is not only unnecessary, but, in fact, disadvantageous, at least, until the precipitate has fully formed. The authors named agree that it is only by working under certain conditions-absence of any large excess of precipitant, of ammoniacal salts, and of ammonia-that a precipitate of normal composition is obtainable (see below). From their labors it would seem clear that the common way of adding the phosphate precipitant to the ammoniacal solution of the magnesium salt is not calculated to produce a precipitate of normal composition. The precipitant should be added to the acid solution of the magnesium., and ammonia should then be added in slight excess.

The precipitate as ordinarily formed differs from the normal in containing relatively more ammonium and less magnesium-for instance, an admixture of such a molecule as $\mathrm{Mg}\left(\mathrm{NH}_{4}\right)_{4}\left(\mathrm{PO}_{4}\right)_{2}$-the result being that when ignited in the ordinary way too much magnesium is found because of formation of some metaphosphate. To obviate this error, Neubauer considers it absolutely necessary to blast the precipitate for

a Zeitschr. angew. Chemie, 1896, p. 435.

b.Am. Jour. Sci., 4th ser., vol. 7, 1899, p. 187; Chem. News, vol. 79, 1899, pp. 233, 244, and 255; Zeitschr. anorg. Chemie, vol. 20, 1899, p. 121. 
half an hour and then to repeat the blasting for a second half hour to see if constant weight has been reached. The phosphate is then entirely pyrophosphate, which is quite unaffected by further blasting. (See, however, B, a, p. 125.) 'The intense heat has caused a decomposition of the metaphosphate with volatilization of $\mathrm{P}_{2} \mathrm{O}_{5}$, as follows: $2 \mathrm{Mg}\left(\mathrm{PO}_{3}\right)_{2}=\mathrm{Mg}_{2} \mathrm{P}_{2} \mathrm{O}_{7}+\mathrm{P}_{2} \mathrm{O}_{5}$. Neubauer worked with the usual excess of ammonia added after the bulk of the precipitate had formed, and it remains to be seen whether by precipitating and working according to Gooch and Austin the composition of the precipitate is always close enough to the ideal $\mathrm{MgNH}_{4} \mathrm{PO}_{4}$ to obviate the necessity for blasting.

Gooch and Austin call attention to a modification proposed long since by Wolcott Gibbs, ${ }^{a}$ whereby the phosphorus and magnesium salts are first boiled together in neutral solution for a few minutes and to the cooled solution ammonia is added. The results are said to be remarkably exact, but $\mathrm{K}$. K. Järvinen ${ }^{b}$ reports to the contrary. This chemist obtained a normal precipitate by either of two methods of his own, of which he regards the following as more convenient and reliable, because ammonium salts exert no influence on the result. It is likewise applicable to the precipitation of phosphoric acid by magnesia mixture.

The hot magnesium solution, containing at least 2 to 3 grams of ammonium salts (to prevent precipitation of magnesium hydroxide) and 20 to $30 \mathrm{~cm}^{3}$ of 10 per cent ammonia solution for 0.4 gram $\mathrm{Mg}_{2} \mathrm{P}_{2} \mathrm{O}_{7}$, is heated to boiling and the precipitant is added slowly. Then more ammonia is added and the solution allowed to cool. A coarsely crystalline precipitate forms which, it is claimed, consists entirely of dimagnesium-ammonium phosphate. This procedure of Järvinen was employed where but a single precipitation of the magnesium was intended, and it does not seem to be adapted for a second precipitation. As this is always called for in rock analysis, it does not appear best to adopt his procedure for our purposes.

E. Raffa ${ }^{c}$ has also studied the conditions under which a precipitate of normal composition is afforded, but his directions will seldom be of use in mineral analysis. They are therefore not repeated here.

\section{b. PRECIPITATION.}

$\alpha$. First precipitation.-This is made without special precautions in the filtrate from the first calcium-oxalate separation (p. 118) by sodium-ammonium-hydrogen phosphate (microcosmic salt) ${ }^{d}$ in indefinite decided excess and without the great excess of ammonia usually prescribed. It is not necessary to first remove ammoniacal salts

a Am. Jour. Sci., 3d ser., vol. 5, 1873, p. 114.

$b$ Zeitschr. anal. Chemie, vol. 44, 1905, p. 335 .

c Gazz. chim. ital., vol. 38 , pt. 2, 1908, p. 556.

d The objection that has been made by one writer to the use of this salt instead of disodium-hydrogen phosphate is, so far as our experience teaches, entirely groundless. 
unless very little magnesium is present, and then only in order to hasten precipitation. The facts that large quantities of these salts retard precipitation and that the composition of the precipitate is affected by them as well as by the way in which the precipitation is made are of importance only when a single precipitation is to be made or in the final of two or more.

Continued agitation of the liquid by a mechąnical stirrer is of advantage in greatly shortening the time of complete precipitation, which is in general shorter the greater the quantity of magnesium present.

$\beta$. Second precipitation.-Platinum sulphide usually strongly contaminates the separated phosphate, but this matters not, as it remains on the filter when the phosphate is redissolved in hydrochloric acid, of which not more than the amount really needed should be used. The solution thus obtained is united with that of the residue from evaporation and.ignition of the second filtrate from the calcium oxalate (10. A. a, p. 118), and is diluted if necessary. A few drops of sodium-ammonium-phosphate solution are now added, and ammonia, drop by drop, in slight excess, with constant stirring till the crystalline precipitate has well formed. Once formed a few cubic centimeters more of ammonia are to be added.

\section{METHODS OF COLLECTING AND IGNITING THE PRECIPITATE.}

a. ORDINARY PROCEDURES.

Were it not for the necessity of examining the precipitate for impurities after ignition (C, p. 126), it were best collected in a Gooch crucible, provided the asbestos felt is well constructed and not of the serpentine variety so largely on the market. It is therefore well to use an ordinary crucible of platinum. Neubauer ignites slowly in platinum after drying, without removing from the paper, and applies the blast only when the carbon has been wholly burned off. ${ }^{a}$ The danger of attack of the platinum by thus igniting the precipitate in contact with the paper is extremely slight, though it has happened once or twice in our experience that a crucible has been ruined by so doing. Why reduction should have occurred in these cases and not in the hundreds of others is not known.

The precipitate, wrapped in its moist paper, is best dried in the crucible itself, the paper then slowly charred without allowing it to ignite, the carbon burned off over a gradually increasing flame, and finally a weak blast applied for a long time and repeated to constant weight to insure volatilization of any excess of $\mathrm{P}_{2} \mathrm{O}_{5}$ over and above that required for the pyrophosphate formula. (See A. a, p. 123.) 
The experience of the Survey chemists was for a time decidedly adverse to the blasting of magnesium pyrophosphate as prescribed by Neubauer, whether obtained by precipitation of magnesium or of phosphate solutions. If the weights were not large the salt fused in part or wholly, and when this occurred reduction seemed to be rapid and the crucible was rather strongly attacked, as shown by its loss in weight after dissolving out the pyrophosphate. Presumably this effect was caused by a blast of higher temperature than that employed by Neubauer, who is very emphatic in his assertion that neither pure pyrophosphate nor crucible suffers the slightest loss when blasted for hours. This seems to be true with a blast of but moderate temperature, giving less than $1,100^{\circ}$ in the crucible. What effect the presence of slight amounts of foreign phosphates may have in increasing the fusibility of magnesium pyrophosphate is unknown. Järvinen found that his precipitates obtained by the Gibbs method fused readily and gave too high results, and further that $\mathrm{P}_{2} \mathrm{O}_{5}$ did seem to volatilize slowly from $\mathrm{Mg}_{2} \mathrm{P}_{2} \mathrm{O}_{7}$ over the blast.

\section{b. MODIFICATION OF ULBRICHT.}

At one time the procedure first recommended by R. Ulbricht, later by $\mathrm{K}$. Broockmann, and also by L. L. de Koninck, was used: It consists in dissolving the ammonium-magnesium phosphate off the filter with nitric acid, collecting the filtrate in a weighed crucible, evaporating the contents to dryness, ${ }^{a}$ and subsequently igniting, the product being presumably pyrophosphate. But it was soon observed that the ignited salt, especially when large in amount, does not always dissolve completely in hydrochloric acid, but that sometimes a white residue is left in light lumps which appear to be quite insoluble in acids. This residue contains no silica, but only the constituents of a magnesian phosphate, and it may be a peculiar metaphosphate. Whether its appearance is due to an abnormal composition of the original magnesian precipitate or to conceivable change during evaporation in the crucible with nitric acid remains to be determined. Until this is done the employment of this method of igniting is not to be recommended.

\section{CONTAMINATION BY AND REMOVAL OF BARIUM, CALCIUM, AND MANGANESE.}

a. BARIUM.

Barium phosphate will not contaminate the second magnesian precipitate unless there are notable amounts of barium in the rock, in which case it must be removed by sulphuric acid prior to the final precipitation of the magnesium.

a A pink color of varying intensity almost invariably becomes apparent as the mass approaches dryness, giving a most delicate test for the traces of manganese which always escape precipitation by ammonium sulphide or bromine. 
b. CALCIUM.

Calcium, however, is probably never absent, and has to be estimated and allowed for as follows:

$\alpha$. Preferred method.-The ignited pyrophosphate is dissolved in a little dilute sulphuric acid and enough absolute alcohol is added to make about 90 to 95 per cent of the final volume. After several hours the sometimes almost invisible precipitate of calcium sulphate is brought onto a small filter and washed free of phosphoric acid with alcohol, dried, and dissolved in hot water slightly acidulated with hydrochloric acid. After rendering the solution ammoniacal and heating, a few crystals of ammonium oxalate are thrown in and the heating is continued till a precipitate becomes visible. In a short time it may be filtered, ignited, and weighed as calcium oxide. Its weight, averaging less than $0.5 \mathrm{mg}$., is to be added to that of the lime already found and subtracted as tricalcium phosphate (not pyrophosphate) from that of the magnesium pyrophosphate, in order to arrive at the true figure for magnesia. The alcoholic filtrate is to be evaporated, ignited to destroy organic matter, and tested for manganese, as under c below.

B. Alternative method.-A less certain way to separate the calcium that is in the pyrophosphate, but one which admits of detecting small amounts of aluminum and iron that may have escaped precipitation earlier in the analysis, is the following:

"To the ignited pyrophosphate, dissolved in but slight excess of hydrochloric acid, is added ammonia to alkalinity, and then acetic acid, drop by drop, till the solution, which should measure 10 to 30 $\mathrm{cm}^{3}$ and not be hot, clears. It now and then happens that a little flocculent matter fails to dissolve. This is to be removed, ignited, and subtracted from the original weight, if proved to be free from magnesium, which it often contains (see c, below). It is likely to consist, in great part or wholly, of phosphates of aluminum, iron, or manganese, and shows often a reddish color on ignition. If an excess of acetic acid has been added, this is cautiously removed by ammonia. Then a drop or two of solution of ammonium oxalate is added, and the small beaker is set aside, for twelve hours, if necessary. Almost invariably a small precipitate shows itself, which, if fine grained and nonadherent to the glass, may be regarded as pure calcium oxalate; otherwise it contains or may largely consist of magnesium oxalate. It is in that case to be collected, ignited, redissolved, and reprecipitated. This separation, to be satisfactory, requires great care.

\section{c. MANGANESE.}

A separation from one another of the iron, aluminum, and manganese which the flocculent precipitate mentioned under the second method in b may contain is not worth the trouble, careful prior treat- 
ment being presupposed. The first two constituents named may have been introduced since the original precipitation of the iron and aluminum, and their amount in terms of those elements is very small. But the amount of manganese it contains may be a sensible proportion of the whole of that element if bromine or ammonium persulphate was not used to secure its removal earlier in the analysis. The precipitate should therefore be brought into nitric or sulphuric acid solusolution. The solution containing the magnesium is also manganiferous. It is to be evaporated to dryness, the volatile salts expelled by ignition, and the residue again evaporated two or three times with a few drops of nitric acid, or once with sulphuric acid, to certainly remove all traces of chlorine, and united with the small solution above mentioned. The manganese is now determined colorimetrically, as in 9.C.b, page 117 , and its amount added to that already found, unless the total has been ascertained from a separate portion of the sample. In any case its amount, if significant, is to be deducted as pyrophosphate from the gross weight of the magnesium pyrophosphate.

The distribution of the manganese over the alumina, lime, and magnesia, when ammonia has been employed for the precipitation of the alumina and no attempt has been made to remove the manganese at all, is shown in the table on page 114 .

\section{LIABILITY TO ERROR IN THE AVERAGE MAGNESIUM DETERMINATION.}

Experience has shown that the majority of analysts are pretty sure to make a very serious plus error in determining moderate amounts of magnesia, such as are found in limestones and Portland cements. The causes of this error will be sufficiently apparent after a careful perusal of the foregoing pages. Everyone ought to make the tests for foreign contamination just described, if only to convince himself of the reality of the error even after very careful work.

\section{TITANIUM.}

\section{A. COLORIMETRIC DETERMINATION WITH HYDROGEN PEROXIDE.}

a. PRINCIPLe OF THE METHOD (WELleR's) $a$ AND THE CONDitions that AFFect it.

The method consists in comparing the color of a known bulk of solution to be tested with that of a standard solution of titanium sulphate, both having been fully oxidized by hydrogen peroxide. The strength of the peroxide should be approximately measured by titration with permanganate on opening a fresh bottle and again after a few weeks, otherwise very serious error may arise through its deterioration.

Mere traces of hydrofluoric acid or a fluoride in either the peroxide or the titanium solution render this method inexact by partly bleach- 
ing the yellow color, ${ }^{a}$ hence care should be exercised as to the character of the peroxide, of which the commercial brands often contain fluorine. (For method of testing see page 40.) For the same reason the peroxide should be free from appreciable traces of phosphoric acid. (See e. r, p. 134.) The "perhydrol" of Merck is a most excellent preparation, though some commercial brands are satisfactory.

Dunnington ${ }^{b}$ has pointed out the necessity for the presence of at least 5 per cent of sulphuric acid in solutions which are to be thus tested for titanium, in order, as he.concluded, to prevent partial reversion to metatitanic acid, which does not give a color with hydrogen peroxide. P. Faber ${ }^{c}$ believes that failure to get full coloration in absence of excess of acid is not due to reversion, but to the presence of a weakly coloring basic sulphate of titanium. (See, however, below as to the effect of alkali sulphates.) He showed that the normal sulphate suffers loss of acid at moderate temperatures and that even in a bisulphate fusion it becomes basic. He explains ${ }^{d}$ the bleaching effect of phosphoric acid or a phosphate as due to the slow formation of a salt of the peroxide. The color can be partly restored by adding sulphuric acid.

If iron is present in the rock or mineral to the extent of several per cent, its color affects the result. (See e, p. 133.)

In absence of fluorine the mineral constituents that are likely to be present in important amounts in the titanium solution undergoing test do not appear to exert any appreciable effect, according to George Steiger, ${ }^{e}$ but in a very recent paper H. E. Merwin ${ }^{f}$ maintains that alkali sulphates bleach markedly unless much sulphuric acid is present. He gives the following data:

\begin{tabular}{|r|r|r|}
\hline $\begin{array}{c}\text { Sulphate } \\
\text { (grams). }\end{array}$ & $\begin{array}{r}\text { Acid } \\
\left.\text { (cm. }^{3}\right) .\end{array}$ & $\begin{array}{c}\text { Bleaching } \\
\text { (per cent). }\end{array}$ \\
\hline & 3 \\
& 6 & $\begin{array}{r}0.5 \\
2.0\end{array}$ \\
8.0 & 15 \\
0.4 & 9 \\
2.9 & 21 \\
8.0 & 14 \\
\hline
\end{tabular}

Now, since alkali sulphates are almost always present in the test solution as ordinarily prepared ( $r$, p. 109) in amounts up to or exceeding 6 grams, he concludes, perhaps without sufficient reason, that all titanium determinations hitherto made by colorimetry are too low.

a Fillebrand, W. F., Jour. Am. Chem. Soc., vol. 17, 1895, p. 718; Chem. News, vol. 72, 1895, p. 158; Bull., U. S. Geol. Survey No. 167, 1900, p. 56.

b Jour. Am. Chem. Soc., vol. 13, 1891, p. 210.

$c$ Chem. Zeitung, 1907, p. 263.

d Zeitschr. anal. Chemie, vol. 46, 1907, p. 277.

e Jour. Am. Chem. Soc., vol. 30, 1907, p. 219.

$f$ Am. Jour. Sci., 4th ser., vol. 28,1909, p. 119. 20091-Bull. $422-10-9$ 
Merwin's statements as to the bleaching effect of alkali sulphates (p. 191) and the counteracting effect of a sufficiency of sulphuric acid have been confirmed.

Merwin has also found that the color intensity is increased by increase of temperature.

In spite of the above observations the colorimetric method gives results that are probably fully equal to those attainable by the best gravimetric method and in much less time, for such percentages as are found in rocks, clays, and soils, usually under 1 per cent, but occasionally rising to 2 or even 3 per cent or more.

b. PREPARATION OF THE STANDARD.

Potassium titanium fluoride, $\mathrm{K}_{2} \mathrm{TiF}_{6}$, best serves as the starting point for the preparation of the standard solution. A quantity of this is recrystallized from boiling water one or more times, dried, and preserved in a glass-stoppered bottle. Enough of it to make one-half to 1 liter of the standard sulphate solution is put into a platinum dish and evaporated several times with strong sulphuric acid without bringing to dryness, till the fluorine is completely expelled. The residue is then taken up with water containing enough sulphuric acid to make at least 5 per cent of the latter when fully diluted. Two 50 to $100 \mathrm{~cm}^{3}$ portions of the prepared solution are then further diluted, boiled, and precipitated with ammonia. The precipitates are collected on paper, washed with hot water till free from alkali, ignited moist in the filter, blasted, and weighed. Duplicates should agree almost exactly. From the weights found the strength of the standard is calculated and the result affixed to the bottle containing it. The stopper of the bottle should be coated with vaseline and the needed quantities of solution should be withdrawn by a pipette, never poured. In' a solution so prepared there is not enough alkali sulphate to weaken the color when peroxidized.

The stock solution so prepared is made to hold as nearly as may be 1 centigram of $\mathrm{TiO}_{2}$ in $10 \mathrm{~cm} .^{3}$. Of this $5 \mathrm{~cm}^{3}$ (or 10 if the larger amount is needed) are mixed with a sufficiency of hydrogen peroxide ( $2 \mathrm{~cm} .{ }^{3}$ of 3 per cent reagent is ample for $10 \mathrm{~cm} .^{3}$ of the standard) and diluted with 5 per cent sulphuric acid in a measuring flask to 50 or $100 \mathrm{~cm}^{3}$, according to the original volume. Each cubic centimeter of the diluted standard will then contain $0.1 \mathrm{mg}$. of $\mathrm{TiO}_{2}$.

\section{c. THE TEST SOLUTION.}

(1) Titanium can be determined, as a rule, most conveniently in the solution which has served for the titration of total iron ( $r$, p. 109). The direct use of this solution involves, however, for accurate work, corrections for its iron and alkali sulphate contents, as given later. 
The effect of the latter can be overcome by an ammonia precipitation, raoderate washing of the precipitate, and its solution in 5 per cent sulphuric acid.

(2) The error accompanying the use of alkali sulphates can also be avoided by making the determination on some other portion of the rock powder than that indicated in the foregoing paragraph. At one time it was the practice in the Survey laboratory to combine it with the determination of barium, as described in Bulletin 148, by decomposing the powder by sulphuric and hydrofluoric acids, ${ }^{a}$ expelling the latter by repeated evaporations with sulphuric acid, taking up with dilute sulphuric acid, ${ }^{b}$ filtering from barium sulphate, etc., and estimating the titanium colorimetrically in the filtrate. The expulsion of fluorine must be thorough, or else the titanium result will be low, as already stated (p. 128), and it is not always easy to effect this complete removal, though the time required to do so seems to be in no slight degree dependent on the nature of the fluorides to be decomposed. Long after every trace of fluorine seems to be gone, the formation of a crust on the evaporating solution sometimes allows an accumulation of enough hydrofluoric-acid gas to become plainly manifest to the smell on breaking the crust.

(3) Both the iron and alkali effects are excluded if the determination is made on the precipitate of $\mathrm{TiO}_{2}$ and $\mathrm{ZrO}_{2}$ obtained as in $\mathrm{B} . \mathrm{b}$ (p. 136). Here; however, errors have to be considered that are incident to prior fusion with sodium hydroxide in a silver crucible and to possible incomplete precipitation of the titanium by one or another of the methods outlined on page 137.

(4) At times the residue from an alkali carbonate fusion of the rock powder, which has been obtained as in 13. A (p. 138), and from which zirconium has been separated (14. A, p. 141) can be used conveniently. The prior removal of zirconium is called for because for the titanium determination there must be far more sulphuric acid present than is permissible for the zirconium separation. However, the conditions here are such as to involve corrections for the iron of the rock, and for phosphoric acid and alkali sulphate also, if: the latter had been added in considerable amounts for the precipitation of zirconium. In the majority of cases the amounts of sodium phosphate needed for two precipitations of the zirconium should produce no sensible error in the value found for the titanium. The precaution should never be omitted, when determining the titanium

\footnotetext{
$a \mathrm{It}$ is to be borne in mind that evaporation with hydrofluoric acid alone results in loss of titanium by volatilization, but that there is no loss if excess of sulphuric acid is also present.

$b$ With acid rocks solution is very complete, and it can be made nearly so with the most basic by transference to a small beaker and gentle boiling. The residue thus obtained may contain, besides barium sulphate, a little calcium sulphate, zircon, andalusite, topaz, and possibly a trace of titanium in some form. It is therefore to be thoroughly fused with sodium carbonate, leached with water, fused with potassium pyrosulphate, dissolved in dilute sulphuric acid, filtered, and the filtrateadded to the main one. The Inşolublẹ mattẹ wịl now bechlefly barium sulphate, for thę further treatment of which see 13. A, pag 138 .
} 
here, to combine the two filtrates from the zirconium phosphate in order to find all the titanium.

The needs of the case or the convenience or preference of the analyst after he has acquired experience will decide which of the above courses to pursue in determining titanium by colorimetry. Naturally, a procedure is to be preferred which will obviate the need for corrections, but in order to secure final conditions which satisfy this aim it were folly to run the risk of incurring other errors of uncertain and varying magnitude when it is possible, though perhaps troublesome, to apply corrections. It is much to be desired, however, that the exact conditions for securing the best results in presence of disturbing agents should be further studied, in order that more positive directions may be formulated than is now possible.

Even if no corrections are applied in rock analysis the error in the titanium value found is so small as to be of rather slight importance, especially if it is known to be always in one direction. Of course, any error incurred involves an equal and opposite one in the value found indirectly for alumina.

\section{d. THE COLOR COMPARISON.}

The sulphate solution containing the titanium, having been evaporated, if necessary, to less than $100 \mathrm{~cm} .{ }^{3}$, is to be fully oxidized with hydrogen peroxide, and if the color is less intense than that of the standard, is made up to $100 \mathrm{~cm}^{3}$ with dilute sulphuric acid in a measuring flask, and mixed; otherwise, in a flask of sufficient size to insure that its color shall be less intense. One of the rectangular glasses (fig. 5, p. 34) being filled with the solution to be tested, $10 \mathrm{~cm}^{3}$ of the diluted standard are run into the other from a burette, and water is added from a second burette until there is no distinction as to color. The temperatures of test solution and standard should be alike. A second and a third portion of the standard can be run in and diluted and the mean of several determinations struck, when a simple calculation gives the percentage of $\mathrm{TiO}_{2}$ in the rock, the amounts in the two solutions being directly as their volumes, provided acid and interfering substances are present in each in like amounts.

If the convenient but expensive Soleil-Duboscq colorimeter is used, or the modification of Steiger or Schreiner (figs. 6-7, pp. 35, 37), or the simple Nessler tubes, it is of course unnecessary to dilute the rock solution to the extent above required, should it be stronger than the standard. Experience has shown, however, that differences can not be sharply estimated in strongly colored solutions, and that the results are much more satisfactory when the color intensity is not much if any greater than that given by a diluted standard of the abeve concentration-that is, $0.1 \mathrm{mg}$. $\mathrm{TiO}_{2}$ per cubic centimeter. 
e. CORRECTION FOR THE COLOR OF FERRIC SULPHATE.

The error introduced by iron, in consequence of the yellowish color of its sulphate solution, is practically negligible unless its percentage is high, say over 5 per cent. Correction should be applied in one of the following ways:

$\alpha$. By allowing for the coloring power of the iron in the test solution.The color of ferric sulphate solution can not be matched closely by that of one containing titanium peroxide, but tests that have been rnade go to show that the coloring effect of 0.1 gram $\mathrm{Fe}_{2} \mathrm{O}_{3}$ in $100 \mathrm{~cm} .^{3}$ of 5 per cent sulphuric acid solution is about equal to $0.2 \mathrm{mg}$. of $\mathrm{TiO}_{2}$ in $100 \mathrm{~cm}^{3}$ when oxidized by hydrogen peroxide. This amounts to a correction of only 0.02 per cent on 1 gram of rock containing the unusual amount of 10 per cent $\mathrm{Fe}_{2} \mathrm{O}_{3}$. The following methods are probably more exact with high iron percentages, though not so expeditious:

B. By addition of iron to the standard.-W. A. Noyes ${ }^{a}$ adds to the standard solution approximately as much ferric iron as will give it, after the colors are matched, the same iron strength as the test solution. This could not be done with the apparatus depicted in figs. 6-7 (pp. 35-37), though practicable with Nessler tubes. In using the Survey colorimeter (fig. 5, p. 34), it would perhaps be best to make first an approximate determination of the titanium in order to find the final volume of the standard after dilution, and then to make an exact determination, having added to a fresh portion of the standard the requisite amount of iron salt. ${ }^{b}$ For all practical purposes it would probably be sufficient to add the requisite amount of iron salt in solid form to the standard after having diluted it nearly enough to match the test solution, and after it has dissolved to proceed with the test. But this procedure has the disadvantage that each comparison would necessitate the weighing out of a separate portion of iron salt, whereas by the following method the number possible would be limited only by the stock of diluted standard and of prepared ferric sulphate solution which serves for its further dilution. The amount to be used per cubic centimeter of diluent is calculated as follows: Let $A$ be the number of cubic centimeters of the test solution, $a$ of the standard used, $b$ of the water needed to dilute the standard in the preliminary test, $p$ the weight of iron in terms of $\mathrm{Fe}_{2} \mathrm{O}_{3}$ in $\mathrm{A}$, and $x$ the weight of iron in terms of $\mathrm{Fe}_{2} \mathrm{O}_{3}$ to be contained in each cubic centimeter of water used for diluting the standard in the final tests. Then $x=\frac{a+b}{A b} p$. It will of course be understood

a Jour. Anal and Appl. Chem., vol. 5, 1891, p. 39.

$b$ Ferric-ammonium alum is most convenient for the purpose, but its use introduces an error due to the bleaching effect of the ammonium sulphate it contains. The amount of this is, however, so small as to give rise to an error that is probably negligible. 
that a new test solution requires the preparation of a new iron solution with which to dilute the standard.

$r$. By the use of phosphoric acid.-The color of a ferric sulphate solution is removed by addition of phosphoric acid. Several observers have, however, noticed that this acid greatly weakens the color of a titanium peroxide solution. This is no objection to its employment in titanium colorimetry, provided a corresponding amount is also added to the standard that serves for comparison. It can be used as the acid or in form of an alkali salt.

A known amount, sufficient to decolorize the iron, is first added to the test solution before complete dilution and the amount to be contained in each cubic centimeter of the water used in the final dilution of the standard can be found like the iron in $\beta$ above.

Both P. Faber, ${ }^{a}$ in the paper already cited on page 129 , and J. H. Walton, jr. ${ }^{b}$ have experimented with the $e_{\iota}$ method and report excellent results. Walton gives data showing the effect of increasing amounts of phosphoric acid in bleaching the titanium color, from which and from corroborative tests made in the Geological Survey laboratory it appears that with highly phosphatic minerals and ores their phosphorus content must be taken into account. The percentages of phosphorus in rocks are too small, even in extreme cases, to affect the color of the titanium that may be present when peroxidized.

f. CORRECTION FOR the EFFECT OF ALKaLI SUlphates.

In order to correct for the bleaching effect of alkali salts, the standard may be diluted with alkali sulphate solution in the same manner as with ferric iron or phosphoric acid in e. $\beta$ and $\gamma$; or Merwin's ${ }^{c}$ corrections may be applied. These vary with the amounts of titanium in the solution, but "in rock analysis, by using 6 grams of pyrosulphate, which is equivalent to 4 grams of normal sulphate, and 2 grams of acid for the melt containing the titanium, and dissolving this in water to which $10 \mathrm{~cm} .{ }^{3}$ of strong sulphuric acid has been added, a nearly negligible correction of only 3 per cent need be added. If the titanium exceeds 0.02 gram, no correction is required. In case the melt is dissolved in $100 \mathrm{~cm}^{3}$ of 5 per cent sulphuric acid, the titanium found-if the amount is between $0.002 \mathrm{gram}$ and 0.01 gram-is too low by approximately 0.0004 gram."

\section{B. GRAVIMETRIC METHODS.}

a. GOOCH'S METHOD.

$\alpha$. Description of the method.-When titanium is present in excess of 4 to 5 per cent and whenever for any reason it is desired to employ a gravimetric method, among the few that have been thoroughly

$a$ Chem. Zeitung, 1907, p. 263.

b Jour. Am. Chem. Soc., vol. 29, 1907, p. 481.

c Am. Jour. Scl., 4th ser., vol. 28, 1909, p. 119. 
tested that of Doctor Gooch ${ }^{a}$ is unequaled. With one or two minor modifications introduced by Dr. T. M. Chatard, ${ }^{b}$ it is as follows:

Any solution of the rock freed from silica can be used, and the first step is to remove the iron. This is best done, after adding tartaric acid and reducing the iron by means of hydrogen sulphide to the ferrous condition, by rendering the solution ammoniacal and introducing more hydrogen sulphide. If the iron is not thus reduced before precipitation, titanium will be in part thrown down also. ${ }^{c}$ The amount of tartaric acid is to be gaged according to the combined weights of the oxides to be held by it in solution, and three times this weight is ample. After removing the iron sulphide by filtration-little washing suffices; because of the relatively small amount of titanium commonly present-the tartaric acid is destroyed as follows:

Potassium permanganate to the extent of two and one-half times the weight of the tartaric acid used is made into a strong solution, and to the ammoniacal filtrate from the iron sulphide enough sulphuric acid is introduced to leave some excess after all the permanganate has been reduced. After expulsion of hydrogen sulphide by boiling, the permanganate is added gradually to the hot solution contained in a large beaker or flask. A vigorous reaction ensues. When a permanent brown precipitate of manganic hydrate appears, the tartaric acid has been fully broken up, and the precipitated manganese is to be redissolved by a few drops of ammonium bisulphite or of sulphurous acid solution.

Ammonia is then added in slight excess, followed at once by acetic acid in considerable excess, and the boiling is continued for a few minutes. Thereby the titanium is freed from most of the alumina, and from lime and magnesia if they had not been earlier removed, also from most of the manganese introduced. The precipitate is filtered and washed with water containing acetic and sulphurous acids, then ignited, fused thoroughly with sodium carbonate, and leached with water to remove phosphoric acid and most of the remaining alumina. The residue is again ignited and fused with sodium carbonate. To the cooled melt in the crucible strong sulphuric acid is to be added, wherein it dissolves readily by aid of gentle heat. This solution is to be poured into a small volume of cold water and the platinum it contains precipitated by hydrogen sulphide at or near boiling temperature. After filtering and cooling, ammonia is added till the titanium is just precipitated, and a measured volume, containing a known weight of absolute sulphuric acid, is then added-just enough to redissolve the precipitate. The solution is then made up with acetic

a Proc. Am. Acad. Arts Sci., n. s., vol. 12, p. 435; Bull. U. S. Geol. Survey No. 27, 1886, p. 16; Chem. News, vol. 52,1885, pp. 55,68 .

b Am. Chem. Jour., vol. 13, 1891, p. 106; Bull. U. S. Geol. Survey No. 78, 1891, p. 87; Chem. News, vol. 63,1891, p. 267.

c Cathrein, A. Zeitschr. f. Kryst., vol. 6, 1882, p. 243; vol. 7, 1883, p. 250. 
acid in such amount that the final bulk shall contain from 7 to 11 per cent of absolute acid, and then enough solid sodium acetate is stirred in to more than take up the sulphuric acid introduced. On rapidly bringing the liquid to ebullition the titanium is precipitated in flocculent and easily filterable condition, and the precipitation is complete after a minute's boiling, provided all the prescribed conditions have been followed and zirconium is absent.

The precipitate is washed first with acetic acid of 7 per cent strength and then with hot water. After fifteen to twenty minutes' ignition over a good burner it is in condition for weighing and will lose no more weight over the blast lamp. For large amounts of titanium a repetition of the sodium-carbonate fusion, etc., should be made. The actual carrying out of all these operations, when once the method is understood, requires much less time than the detailed description would indicate.

$\beta$. Gooch's method not directly applicable to rocks containing zirconium.-Prior to the adoption of the colorimetric method, Doctor Gooch's was invariably used in this. laboratory. Occasional inability to secure clean and complete precipitation by it was experienced, especially with a certain series of rocks rather poor in titanium. Long research showed the difficulty to be due to the presence of zirconium, which acts as a marked preventive of the precipitation of titanium by boiling in an acetic-acid solution under the conditions of the Gooch method.

The above rocks were found to contain up to 0.2 per cent of $\mathrm{ZrO}_{2}$, and this amount was able to prevent precipitation of 0.3 per cent of $\mathrm{TiO}_{2}$. The titanium which came down in excess of this amount did not settle out in flocculent condition, as happens when zirconium is not present, and it was difficult to filter. After the removal of the zirconium, however, in the manner to be hereafter described (14. A, p. 141), no difficulty was experienced in precipitating all the titanium with the usual ease.

\section{b. METHODS OF DITTRICH AND FREUND.}

In recent papers M. Dittrich and S. Freund ${ }^{a}$ have proposed the several methods below, involving no new principles, by which they separate-completely, so they claim-titanium and zirconium from iron as well as manganese. These methods, except, perhaps, the third, presuppose, in rock analysis, the prior separation of aluminum and phosphorus by fusion with sodium hydroxide in a silver crucible (c, p. 112). Attention has been directed (8. C. b, p. 111) to the error resulting from attempting to separate aluminum from titanium by either fused or dissolved sodium hydroxide in absence of appreciable 
amounts of iron and to the fact that when iron is present in some amount this error does not seem to arise.

If such a fusion has been made the insoluble residue may be dissolved in sulphuric or hydrochloric acid, according to the method that is to be followed, or a bisulphate fusion of the ignited oxides may be employed if alumina is absent. Care must be taken to remove all silver derived from the silver crucible by the alkali fusion.

(1) Titanium and zirconium are precipitated free from iron, provided this is kept wholly in the ferrous state by boiling with sodium acetate under carefully prescribed conditions.

(2) Under proper conditions titanium and zirconium are also precipitated free from iron by sodium thiosulphate.

(3) Titanium and zirconium are separated from all but a trace of iron by boiling with sulphurous acid in a stream of carbon dioxide after prior reduction of the iron in hydrochloric acid solution and careful neutralization of the acid by ammonia. This is merely a modification of the method of Baskerville ${ }^{a}$ to maintain (as in 4 below) complete reduction of the iron. Baskerville's method, as applied at one time by me to the analysis of titaniferous iron ores, yielded after one precipitation about 0.0025 gram of $\mathrm{Fe}_{2} \mathrm{O}_{3}$ in a weight of 0.08 to 0.10 gram $\mathrm{TiO}_{2}$.

(4) The authors regard the following method as relatively the simplest: The sulphate solution is reduced by hydrogen sulphide, filtered into a $750 \mathrm{~cm} .{ }^{3}$ Jena flask provided with a stopper carrying inlet and outlet tubes and a separatory funnel. The solution is now neutralized with sodium carbonate, the formation of ferrous sulphide serving as indicator, a few drops of sulphuric acid are added, and hydrogen sulphide is passed in to complete the reduction of theiron. The gaseous reagent is then wholly removed by carbon dioxide at boiling heat, whereupon a concentrated solution of 5 to $10 \mathrm{~cm} .^{3}$ of ammonium sulphate is allowed to flow in, followed by water, if needed, to make the volume 400 to $500 \mathrm{~cm}^{3}$. With continued passage of carbon dioxide and renewal of escaping water the solution is now boiled for about an hour, then cooled. The white precipitate is filtered and washed with hot water slightly charged with hydrogen sulphide. A few drops of sulphuric acid and hydrogen peroxide are used to cleanse the walls of the flask, and the titanium and zirconium thus dissolved are precipitated by ammonia and added to the main portion, the whole being ignited in platinum over the blast to constant weight. The oxides are fused with potassium bisulphate; silica, derived from the reagents or apparatus, is separated (f, p. 105); and titanium and zirconium are determined, the one colorimetrically, the other by difference. In view of the very small amounts of zirconium in rocks, and also of the 
author's analytical data, it is far preferable to find it directly (14. A, p. 141) instead of by difference. ${ }^{a}$

In the filtrate from the oxides Dittrich and Freund precipitate iron and manganese by ammonia and hydrogen peroxide, and then, presumably as in earlier directions of Dittrich, determine the manganese in this precipitate (after getting the whole into solution) by digesting the sulphate solution with hydrogen peroxide, filtering, and repeating the precipitation.

\section{SUPERIORITY OF THE COLORIMETRIC AND GOOCF METHODS OVER THE OLDER ONES.}

In view of the good results obtainable by the colorimeter method in most cases, and by the Gooch method in the absence of zirconium, it is inexplicable that the old method of precipitation by many hours' boiling in a nearly neutral sulphate solution in presence of sulphurous acid should still find adherents in any part of the world.

\section{BARIUM (ZIRCONIUM, RARE EARTHS, TOTAL SULPHUR, CHROMIUM).}

These five constituents can with great economy of time and labor be determined in the same portion of rock powder, of which a 2-gram portion should be used, though if vanadium is to be looked for it will be best to determine it and chromium in a different portion (18. C, p. 150). It has been said above (10. C, p. 120) that only in very exceptional cases will barium be found with the calcium and strontium after two or possibly three precipitations of the latter as oxalate, since it passes, into the filtrates with the magnesium, whence it may be obtained as sulphate after removal of ammoniacal salts. Addition of some alcohol insures also the recovery of traces of strontium if the rocks are very rich in it; but it is unsafe to regard the amount of barium thus separated from the magnesium as representing the total amount in the rock. It will almost always be found lower than the truth, probably for the reason that there are opportunities during the analysis for slight losses.

\section{A. THE GENERAL METHOD.}

Decomposition is effected by fusing the 2-gram portion with sulphurfree sodium carbonate and an amount of niter insufficient to injure the crucible (one-half to 1 gram), first over the Bunsen flame, then over the inclined blast, the crucible being fitted snugly into a hole in a disk of platinum foil or asbestos board (fig. 3, p. 32) to prevent access of sulphur from the gas flame. In case sulphur is not to be regarded, the niter and disk are omitted. After thorough disintegration of the

$a$ The new method of Dittrich and Freund (Zeitschr. anorg. Chemie, vol. 56, 1907, p. 344), by which zirconium is precipitated as the salicylate and ignited to the oxide, may find application here. 
melt in water, to which a drop or two of methyl or ethyl alcohcl has been added for the purpose of reducing manganate, the solution is filtered and the residue washed with a very dilute solution of sodium carbonate free from bicarbonate. This is to prevent turbid washings. A yellow color in the filtrate indicates chromium.

The filtrate contains, after reduction of the manganate by alcohol, silica, alumina, and all the other acid constituents except titanium, also a very little iron, but the separation of these from the remaining constituents is not always quantitative, never so as to silica and alumina. The proportions of these two which go into the aqueous extract vary greatly according to their relative proportions in the original material and the amounts and character of the bases other than alumina. For instance, with alkali feldspars much of each dissolves, but with pure kaolinite very little of either. With most rocks both filtrate and residue contain large amounts of both.

(For the further treatment of the filtrate see "Sulphur," 24. C, p. 198, and "Chromium," 17. B, p. 147.

The residue is washed from the paper into a small beaker without removing the filter from the funnel and is digested with little more than enough warm dilute sulphuric acid to effect solution of all soluble constituents (stronger acid may be used and in larger amount if barium only is sought). A few drops of sulphurous acid are added at the same time to effect solution of the brown hydroxide of manganese. There will remain undissolved more or less residue. Care must be taken not to digest so long with acid as to cause gelatinization of the dissolved silica. The liquid is passed through the original filter and collected in an Erlenmeyer flask of 100 to $150 \mathrm{~cm}^{3}$. The paper with its contents after washing is ignited, evaporated with hydrofluoric and sulphuric acids together, and the final residue taken up with a little hot dilute sulphuric acid. All the barium will remain undissolved, besides some of the strontium and perhaps a good deal of calcium. This is collected on a small filter and the filtrate added to the former one, which now contains all the zirconium and rare earths. (For its further treatment see 14. A, p. 141, and 15. A, p. 143.)

The ignited residue last obtained is fused with sodium carbonate, leached with water, and the residue dissolved off the filter by a few drops of hydrochloric acid, from which solution the barium is thrown out by a large excess of sulphuric acid. A single solution of the ignited barium sulphate in concentrated sulphuric acid and reprecipitation by water suffices to remove traces of calcium which might contaminate it if the rock was one rich in calcium, and even strontium is seldom retained by it in quantity sufficient to give concern. Should this be the case, however, which will occur when the $\mathrm{SrO}$ and $\mathrm{BaO}$ are together in the rock in, roughly speaking, 0.2 and 0.4 per cent respectively, the only satisfactory way is to convert the sulphates into 
chlorides and to apply to the mixture the ammonium-chromate method of separation (10. D, p. 120).

Barium and strontium sulphates can be brought into a condition for testing spectroscopically by reducing for a very few moments the whole or part of the precipitate on a platinum wire in the luminous tip of a Bunsen burner, then moistening with hydrochloric acid, and testing in the usual manner.

The procedure outlined in the foregoing paragraphs for the estimation of calcium, strontium, and barium in silicate rocks is the one which long experience has shown to be best adapted for securing the most satisfactory results with a minimum expenditure of time. ${ }^{a}$ Even where no attempt is made to separate contaminating traces of strontium and barium one from the other, the error is usually of no great consequence, for an absolute error of even 25 per cent in a substance constituting only 0.1 or 0.2 per cent of a rock is ordinarily of small moment compared with the ability to certify to its presence with approximate correctness.

With such small amounts of barium as are usually found in rocks it is doubtful if Mar's ${ }^{b}$ method for the separation of barium from calcium and magnesium by the solvent action of concentrated hydrochloric acid mixed with 10 per cent of ether on the chlorides could be conveniently applied here, although for larger amounts the method would seem to be accurate and easily executed. Moreover, it would probably not entirely remove contaminating strontium, and hence offers no advantage.

\section{B. THE METHOD WITHOUT REGARD TO ZIRCONIUM AND SULPHUR.}

If zirconium and sulphur are not to be looked for, the simplest procedure is to decompose the powder by sulphuric and hydrofluoric acids (see c. 2, p. 131, under "Titanium"), and to complete the purification of the barium sulphate thus obtained in the manner described in the fourth paragraph of $\mathrm{A}$ above.

\section{ZIRCONIUM.}

This element is rarely looked for by chemists, though shown by the microscope to be one of the most constant rock constituents, usually in the form of zircon, in which occurrence its amount can be approximately judged and a chemical test rendered almost unnecessary; but sometimes it occurs in other minerals, and is then unrecognizable under the microscope. It may in most exceptional cases be present up to a few per cent of the rock, rarely reaches 0.2 per cent, and is usually much less than 0.1 per cent.

$a$ For details consult Hillebrand, W.F., Jour. Am. Chem. Soc., vol. 16, 1894, p. 83; Chem. News, vol. 69, 1894, p. 147.

b Am. Jour. Sci., 3d ser., vol. 43, 1892, p. 521. 


\section{A. AUTHOR'S METHOD.}

For its detection and estimation in such cases, or whenever a search for it seems called for, the following procedure, based on a method used by G. H. Bailey, ${ }^{a}$ has been devised, which serves, when carried out with care, to detect with certainty the merest trace- -0.02 per cent, for instance-in 1 gram.

The preliminary treatment of the rock powder has been fully given under "Barium" (13. A, p. 138), where the separation from barium has been described and also the concentration of the zirconia in a small amount of very dilute sulphuric solution. This should probably not contain much above 1 per cent of sulphuric acid, though the actually permissible limit has not been established. To the solution, which should be in a small flask, is now added hydrogen peroxide to oxidize the titanium, and then a few drops of a soluble orthophosphate solution. The flask is set aside in the cold for twenty-four to fortyeight hours. If the color bleaches after a time, more hydrogen peroxide should be added. Under these circumstances the zirconium is thrown out as phosphate and collects as a flocculent precipitate, which at this stage is not always pure. ${ }^{b}$ (For the treatment of the filtrate see "Rare-earth metals," 15.A, p. 143.) No matter how small or insignificant, it is collected on a filter, ignited, fused with sodium carbonate, leached with water, the filter again ignited, fused with very little potassium or sodium pyrosulphate, brought into solution in hot water with a few drops of dilute sulphuric acid, poured into a flask or beaker of about $20 \mathrm{~cm} .^{3}$ capacity, a few drops of hydrogen peroxide and of sodium phosphate are added, and the flask set aside. Titanium is now almost never present, and the zirconium soon appears as a colorless flocculent precipitate, which can be collected and weighed as phosphate. For the small amounts usually met with it is safe to assume that it contains 50 per cent of $\mathrm{ZrO}_{2}$ (51.8 by theory). If the arnount is rather large, it may be safer to fuse with sodium carbonate, leach, ignite, fuse with pyrosulphate, reprecipitate by ammonia, and weigh as $\mathrm{ZrO}_{2}$. Certainty as to its identity can be had by again bringing it into solution, precipitating by ammonia, dissolving in hydrochloric acid, evaporating to a drop or two, and testing with turmeric paper or by a microchemical reaction. With the very smallest amounts no color can be obtained by this turmeric-paper test, which, however, responds readily to as little as 1 milligram of dioxide and with proper care for as small an amount as 0.3 milligram (Dr. H. N. Stokes). No element other than thorium is ever likely to contaminate the zirconium thus precipitated.

a Jour. Chem. Soc., vol. 49, 1886, pp. 149, 481.

$b$ If the rock contains much iron the precipitate may contain or consist largely of a ferric phosphate. Titanium may also be precipitated to some extent as a phosphate. The degree of such precipitation will be greater as the acidity of the solution is less. 
In Bailey's experiments the precipitation was not made by addition of a phosphate, but is said to be due solely to the hydrogen peroxide, the precipitate being a hydrated peroxide, $\mathrm{Zr}_{2} \mathrm{O}_{5}$, or $\mathrm{ZrO}_{3}{ }^{a}$ My own efforts as well as those of others to secure a precipitate in acid solutions of zirconium sulphate by hydrogen peroxide alone have been unsuccessful, except in very concentrated solutions and with 30 per cent peroxide. The ability to obtain the zirconium free from phosphoric acid would certainly be a great improvement on the method described above.

Were it not for the necessity of precipitating the zirconium in a weakly instead of strongly acid solution, its separation could be made in the same portion in which the titanium is colorimetrically determined.

\section{B. OTHER METHODS.}

G. Streit and B. Franz ${ }^{b}$ claim to secure complete separation of titanium from iron and zirconium by boiling the neutralized solutions of the sulphates with a large excess (50 per cent) of acetic acid. The method has been from time to time recommended, but without any data showing its value. The single separation made by Streit and Franz was far from perfect.

J. T. Davis ${ }^{c}$ separated zirconium sharply from aluminum, but not from iron, by precipitation as an oxyiodate in a boiling neutralized solution of chlorides, but the method is hardly applicable for rock analysis.

C. Baskerville ${ }^{d}$ has proposed a method for the separation of zirconium from iron and aluminum similar to his method for the separation of titanium from those elements (12. B. b. 3, p. 137). It is based on the precipitability of zirconium by boiling the neutralized chloride solution for two minutes in presence of sulphurous acid, and seems to be excellent. As titanium is always present and'is quantitatively thrown down also, the two would have to be separated by hydrogen peroxide and a soluble phosphate. No tests as to the "uavailability of the method for separating the small amounts met with in rock analysis have been made, but M. Dittrich and S. Freund have used it successfully in slight modification for larger amounts (b. 3, p. 137), and have also devised a method for the direct separation of zirconium from titanium by precipitation with ammonium salicylate. ${ }^{c}$

${ }_{a}$ Chem. News, vol. 60,1889, p. 6 .

b Jour. f. prakt. Chemie, vol. 108, 1869, p. 65.

c Am. Chem. Jour., vol. 11, 1889, p. 27.

$d$ Jour. Am. Chem. Soc., vol. 16, 1894, p. 475; Chem. News, vol. 70, 1894, p. 57.

e Zeitschr. anorg. Chemie, vol. 56, 1907, p. 344. 
For the cases in which it may be necessary. to look for rare earths other than zirconia, the following procedures will prove satisfactory and not at all difficult: ${ }^{a}$

\section{A. USUAL METHOD.}

The filtrate from the zirconium phosphate (14. A, p. 141) or, if zirconium is not to be determined, the earlier solution (13.A, p. 138) is treated with excess of potassium hydroxide to precipitate the rare earths and ferric and titanic oxides, while retaining the silica and alumina in solution. After settling, the liquid is decanted and the precipitate is washed once or twice by decantation and slightly on the filter. The precipitate is washed from the paper into a small platinum dish, treated with hydrofluoric acid, and the liquid evaporated nearly to dryness. A little water with a few drops of hydirofluoric acid is added and the insoluble rare-earth fluorides (crude) collected on a small filter held by a perforated platinum or rubber cone and washed with water acidified with the same acid. The precipitate is washed into a small platinum dish and evaporated to dryness with sulphuric acid, the paper being burned and added before expulsion of the acid. The sulphates are dissolved in dilute hydrochloric acid, the earth hydroxides precipitated by ammonia, redissolved in hydrochloric acid, the solution evaporated to dryness, and the residue heated with a few drops of a solution of oxalic acid. Anything that may be present except the rare earths dissolves readily, these remaining as insoluble oxalates. If there is a doubt as to the complete separation of the alkaline earths the ignited oxalates may be dissolved in hydrochloric or nitric acid, precipitated by ammonia, redissolved in whichever one of the acids may have been used, the solution evaporated to dryness, the oxalates reprecipitated, ignited, and the resulting oxides weighed.

The amounts are too small to permit separation into further groups, but the presence of cerium can be readily shown if a strong yellow color develops on heating the ignited oxides with strong sulphuric acid.

\section{B. ALTERNATIVE METHOD.}

The rock powder is thoroughly decomposed by several partial evaporations with hydrofluoric acid, the transparent precipitate of silico-fluorides and of the fluorides of all earth metals except zirco-

a M. Dittrich (Ber. Deutsch. chem. Gesell., vol. 41, 1908, p. 4373) calls attention to the fact that ferric, aluminum, and chromic, as well as uranic, compounds prevent precipitation of cerium, and presumably other rare-earth metals, as oxalate, unless a very large excess of the precipitant is used, but that with such excess precipitation is complete. Salts of the above metals even dissolve cerium oxalate, but excess of oxalic acid or ammonium oxalate throws iț out again. Thiș behạviọr need hardly be conșidered in the procedures here given, 
nium is collected on a platinum cone, washed with water acidulated by hydrofluoric acid, washed back into the dish or crucible, and evaporated with enough sulphuric acid to expel all fluorine. The filter is burned and added. By careful heating the excess of sulphuric acid is removed and the sulphates are taken up by dilute hydrochloric acid. The rare earths, with perhaps some alumina, are then precipitated by ammonia, washed, redissolved in hydrochloric acid, and evaporated to dryness, then taken up with water and a drop of hydrochloric acid, and only enough ammonium acetate to neutralize the latter added, followed by oxalic acid (not ammonium oxalate, which would fail to precipitate thorium). In this way as little as 0.03 per cent of rare earths has been found when working on not more than 2 grams of material.

\section{PHOSPHORUS.}

It is sometimes possible to extract all phosphorus from a rock by simple digestion with nitric acid, but quite as often, if not oftener, this fails; hence the necessity for resorting to one of the longer methods of extraction detailed below. Whatever method is used, great care is required in order to secure accurate results.

\section{A. PROCEDURE WHEN MATERIAL IS AMPLE.}

a. WASHINGTON'S METHOD OF PRELIMINARY TREATMEN'T.

About 1 gram of rock powder is decomposed in a platinum dish or capacious crucible with dilute nitric and hydrofluoric acids. When all gritty particles have disappeared the solution is evaporated to dryness and reevaporated with nitric acid two or three times to decompose. fluorides and silicofluorides to a considerable extent. ${ }^{a}$ The residue is treated on the bath with dilute nitric acid and eventually filtered from any undissolved matter. This last should be for precaution's sake always ignited, fused with a little sodium carbonate, extracted with water, the extract acidified with nitric acid and added to the main solution. (For further treatment see c, below.)

\section{b. ALTERNATIVE METHOD OF PRELIMINARY TREATMENT.}

Fuse with sodium carbonate, separate silica by a single evaporation with nitric acid, treat the ignited silica with hydrofluoric and nitric acids, evaporate to expel hydrofluoric acid, repeat the evaporation with nitric acid alone two or three times, bring the small residue into solution by boiling with nitric acid and add it to the main portion.

'a The method as originally described in Washington's Manual of the Chemical Analysis of Rocks has been modified by Doctor Washington and myself independently to the extent of doing away with the filtration of the precipitate of silicofluorides and fluorides before proceeding with the evaporation. 
C. SUBSEQUENT TREATMENT,

Add to the solution obtained in $\mathrm{a}$ or $\mathrm{b}$ ammonium nitrate and molybdate solution as ordinarily prescribed.

The turbidity often observed on dissolving the precipitated and washed phosphomolybdate in ammonia is due to a compound of phosphorus. If the addition of a small fragment of a crystal of citric or tartaric acid fails to dissolve it, this should always be re-fused with sodium carbonate, extracted with water, and the filtrate otherwise treated as above, in order to secure the phosphorus in it.

According to F. A. Gooch and M. Austin ${ }^{a}$ in order to secure a magnesium-ammonium phosphate of normal composition, the procedure at this point should be as follows: To the phosphate solution, containing not more than 5 to 10 per cent of ammonium chloride and a slight excess of magnesia mixture, a little ammonia is added, and the precipitate is washed in due time with weak ammonia water. In general, however, as these conditions can seldom be fulfilled, they recommend to decant the supernatant liquid through the filter which is later to receive the precipitate, to dissolve this in as little hydrochloric acid as possible, to reprecipitate by dilute ammonia without further addition of magnesia mixture, and to wash finally with weakly ammoniacal water. Excess of ammonia, of ammonium salts, and of precipitant are all objectionable. In rock analysis the second precipitation will seldom be necessary. (For ignition, etc., of the precipitate, see this subject under "Magnesium," 11. B. a, p. 125.)

\section{B. PROCEDURE WHEN MATERIAL IS SCANTY.}

The following procedure admits of determining in the same portion, besides phosphorus, barium, iron, vanadium, chromium, and titanium, the last two either colorimetrically or gravimetrically, and is in large part extracted from a paper by Dr. T. M. Chatard. ${ }^{b}$

Silica is removed by hydrofluoric and sulphuric acids, excess of fluorine expelled, the residue brought into solution as far as possible with sulphuric or hydrochloric acid and hot water, filtered, the residue ignited, fused with sodium carbonate, dissolved in hydrochloric acid, and the solution, after precipitation of barium, added to the main one, which is now precipitated by ammonia to get rid of the magnesium salts usually present and thus insure a cleaner subsequent fusion with sodium carbonate.

The precipitated $\mathrm{Al}_{2} \mathrm{O}_{3}, \mathrm{P}_{2} \mathrm{O}_{5}, \mathrm{Cr}_{2} \mathrm{O}_{3}, \mathrm{Fe}_{2} \mathrm{O}_{3}$, and $\mathrm{TiO}_{2}$ are dissolved in hot hydrochloric acid and filtered into a large platinum crucible, the filter burned and added, the solution evaporated to pastiness, a little

a Am. Jour. Sci., 4th ser., vol. 7, 1899, p. 187; Zeitschr. anorg. Chemie, vol. 20, 1899, p. 121; Chem. News, vol. 79,1899 , pp. $233,244,255$.

b Am. Chem. Jour., vol. 13, 1891, p. 106; Bull. U. S. Geol. Survey No. 78, 1891, p. 87; Chem. News, vol. 63, 1891, p. 267.

20091-Bull, 422-10-10 
water added to dissolve the salts, and dry sodium carbonate added in portions and stirred in thoroughly to prevent lumpiness in the fusion to follow, which is continued for half an hour. Addition of sodium nitrate is not necessary.

The fused mass is boiled out with water and washed with very dilute sodium-carbonate solution. In the residue iron and titanium can be determined by the methods already described. In the filtrate chromium can be determined colorimetrically if present in sufficient amount to give a pronounced color. (See 17. B, p. 147.) Afterward, or immediately if the chromium is not to be thus estimated, enough ammonium nitrate is added to react with all the carbonate and the solution is digested on the bath till most of the ammonium carbonate is gone. Nearly if not quite all alumina is thus thrown out, carrying with it all phosphorus. The precipitate is washed with dilute ammonium-nitrate solution till the yellow color wholly disappears, after which it is dissolved in nitric acid and the phosphorus thrown out by molybdate solution. The filtrate from the aluminum and phosphorus, containing chromium and vanadium, can be treated as detailed in the following sections. .

\section{CHROMIUM.}

If vanadium is absent, or nearly so, as is apt to be the case in those highly magnesian rocks (peridotites), which usually carry a good deal of chromium, the following separation and gravimetric method for chromium gives good and concordant results, but in the presence of vanadium, and it is best generally to assume its presence, the colorimetric method should always be adopted.

\section{A. GRAVIMETRIC METHOD.}

Having obtained chromium in solution as chromate and free from all else but a little alumina, as at the conclusion of the preceding section on phosphorus, proceed as follows:

Concentrate if necessary and add fresh ammonium sulphide, or introdüce hydrogen sulphide. The chromium is reduced and appears as a precipitate of sesquioxide mixed with the rest of the alumina. This precipitate is now treated according to $\mathrm{H}$. Baubigny ${ }^{a}$ by dissolving in nitric acid, evaporating nearly to dryness, and heating with strong nitric acid and potassium chlorate, finally evaporating to dryness to get rid of the acid. Oxidation is complete and very speedy. On dilution with cold water, acid sodium carbonate is added in slight excess, and after two or three hours the precipitated alumina is filtered off. From the filtrate the chromium is then thrown out by fresh ammonium sulphide, redissolved, reprecipitated to free from alkali, and weighed. 
The separation of aluminum from chromium by hydrogen peroxide in ammoniacal solution, as recommended by $P$. Jannasch and E. v. Cloedt, ${ }^{a}$ has been shown by C. Friedheim and E. Brühl ${ }^{b}$ to be valueless.

\section{B. COLORIMETRIC METHOD.}

For this very accurate and by. far the quickest method ${ }^{c}$ for determining chromium in rocks and ores where the amount does not exceed a few per cent there is needed the aqueous extract of a sodium-carbonate fusion of the rock (as obtained, for instance, under "Phosphorus," B, p. 145) in order to compare its color with that of a standard solution.

¿. Preparation and strength of standard solution.-This standard solution is made by dissolving 0.25525 gram or double that amount of pure potassium monochromate in 1 liter of water made alkaline by a little sodium carbonate. Each cubic centimeter then corresponds to 0.1 or $0.2 \mathrm{mg}$. of chromic oxide $\left(\mathrm{Cr}_{2} \mathrm{O}_{3}\right)$, in which condition chromium is usually reported in rocks and ores. It is probably inadmissible to increase the strength of the standard much above the figure given.

$\beta$. Preparation of the test solution.-Before filtering the aqueous extract of the sodium-carbonate fusion a few drops of alcohol (ethyl or methyl) are added to destroy the color of sodium manganate. If the yellow color of the filtrate is very faint, concentration by evaporation will strengthen it, and less than $2 \mathrm{mg}$. of chromic oxide in 1 gram of rock can then be exactly measured. For smaller amounts it is best to employ from 3 to 5 grams of powder and then to concentrate the chromium by precipitation by mercurous nitrate, as detailed in the next section under "Vanadium" (18. C, p. 150); otherwise it may be difficult or impossible, because of the large amount of alkali carbonate present, to obtain a filtrate of sufficiently small bulk to show a decided color.

If niter has been used in the fusion and the crucible has been at all attacked by it, a yellow coloration of the filtrate may be due to dissolved platinum, but neither the proportion of niter nor the temperature of the blast should ever be high enough to permit the crucible to be attacked. A passing yellowish coloration of the filtrate, due to this or some other cause, is not to be ascribed to chromium.

r. Comparison of colors.-The final solution is transferred to a graduated flask of such size that its color shall be weaker than that of the standard chromium solution. Definite amounts of the latter

a Zeitschr. anorg. Chemie, vol. 10, 1895, p. 402.

$b$ Zeitschr. anal. Chemie, vol. 38,1899 , p. 681 .

c Hillebrand, W. F., Jour. Am. Chem. Soc., vol. 20, 1898, p. 454; Chem. News, vol. 78, 1898, pp. 227, 239 ; Bull. U. S. Geol. Survey No. 167, 1900, p. 37. First applied by L. de Koningh (Nederl, Tyds. voor Phirm., Chem. en Tox., 1889) for the estimation of chromium in foodstuffs. 
are then diluted with water from a burette until of the same strength as the test solution, exactly as described on page 34 . For very minute amounts it may be necessary to use Nessler tubes, as in ammonia estimations, instead of the glasses and apparatus there described and depicted.

As with colorimetric methods in general, this one gives better results with small than with large percentages of chromium, yet it can be applied in the latter cases with satisfactory results.by making a larger number of consecutive comparisons with the same solution. ${ }^{a}$

\section{A FEW COMPARATIVE DATA.}

A few comparisons between colorimetric and gravimetric determinations of chromium are here given to show the order of agreement, the former having been made several months and even years after the latter.

\section{Percentage determinations of chromium $\left(\mathrm{Cr}_{2} \mathrm{O}_{3}\right)$.}

\begin{tabular}{|r|c|}
\hline Gravimetric. & Colorimetric. \\
\hline Trace. & 0.018 \\
0.05 & .051 \\
.14 & .12 \\
.08 & .083 \\
Trace. & .013 \\
None. & .0086 \\
None. & .0067 \\
\hline
\end{tabular}

The outcome was somewhat surprising, for it was hardly to be expected that the long and laborious quantitative separations should have resulted so well. It should be mentioned that for the gravimetric tests but 1 or 2 grams at most were used, which accounts for the reported absence of chromium in two instances, this report being based on the lack of color in the aqueous extract of the alkali fusion after removal of manganese.

\section{VANADIUM (CHROMIUM) AND MOLYBDENUM.}

\section{A. DISTRIBUTION OF VANADIUM AND MOLYBDENUM.}

The wide distribution of vanadium throughout the earth's crust has in recent years been clearly established (see pp. 20-21), not only in ores and in coals, but in clays, limestones, sandstones, and igneous rocks. ${ }^{b}$ I have shown ${ }^{c}$ that vanadium occurs in appreciable amounts in the more basic igneous and metamorphic rocks up to

$a$ D. W. Horn has studied the "Variable sensitiveness in the colorimetry of chromium." See Am. Chem. Jour., vol. 35, 1906, p. 253; vol. 36, 1906, p. 196.

$b$ Hillebrand, W. F., Distribution and quantitative occurrence of vanadium and molybdenum in rocks of the United States: Am. Jour. Sci., 4th ser., vol. 6, 1898, p. 209; Chem. News, vol. 78, 1898, p. 216; Bull. U. S. Geol. Survey No. 167, 1900, p. 49.

$c$ Loc. cit. 
0.08 per cent or more of $\mathrm{V}_{2} \mathrm{O}_{3}$, but that it seems to be absent or nearly so from the highly siliceous ones. Some of their ferric aluminous silicate constituents carry still higher percentages-up to 0.13 per cent $\mathrm{V}_{2} \mathrm{O}_{3}$ in a biotite separated from a pyroxenic gneiss. Molybdenum, on the other hand, appears to be confined in quantities susceptible of detection to the more siliceous rocks, and except perhaps in rare instances is not present in them in quantitatively determinable amount when operating on 5 grams of material. Hence the quantitative search for vanadium will usually be limited to rocks with less than 60 per cent of silica. The search for it even then will perhaps not often warrant the necessary expenditure of time, but in this connection it is to be remembered that neglect to determine it introduces an error in the figures for both ferrous and ferric oxides, which in extreme cases may be of considerable moment (see 8. B. b, p. $109 ; 19$. D. c. $\beta$, p. 166).

\section{B. CONDITION OF VANADIUM IN ROCKS.}

The connection below and elsewhere mentioned of vanadium with the ferric aluminous silicates of rocks, taken in connection with the existence of the mineral roscoelite, classed as a vanadium mica, indicates that the vanadium corresponds in 'condition to aluminum and ferric iron, and that it is to be regarded as replacing one or both of these elements. Hence it should be reported as $\mathrm{V}_{2} \mathrm{O}_{3}$ and not as $\mathrm{V}_{2} \mathrm{O}_{5}$.

What its condition may be in matter of secondary origin, like clays, limestones, sandstones, coals, and ores of iron, is yet open to discussion. It was my opinion at one time that it should be regarded as in the pentavalent state $\left(\mathrm{V}_{2} \mathrm{O}_{5}\right)$, but my work on certain remarkable vanadiferous sandstones ${ }^{a}$ of western Colorado, in which it unquestionably occurs as trivalent vanadium $\left(\mathrm{V}_{2} \mathrm{O}_{3}\right)$, has led to a decided unsettling of this view. It is proper to recall that C. Czudnowicz, ${ }^{b}$ because of the extreme difficulty in completely extracting it from iron ores by an alkali-carbonate fusion and because of the easy reducibility of vanadic acid by ferrous salts, under the conditions in which brown iron ores are supposed to form, considered the vanadium in such ores to be in a lower condition of oxidation $\left(\mathrm{V}_{2} \mathrm{O}_{3}\right)$. O. Lindemann' ${ }^{c}$ contrary conclusion with regard to certain iron ores, because the vanadium was extracted as $\mathrm{V}_{2} \mathrm{O}_{5}$ by sodium-carbonate fusion without niter, is not valid, since this would probably be the case even if it existed in the ore as $\mathrm{V}_{2} \mathrm{O}_{3}$.

\footnotetext{
a Hillebrand, W. F., and Ransome, F. L., Am. Jour. Sci., 4th ser., vol. 10, 1900, p. 120; Bull. U. S. Geol. Survey No. 262,1905, p. 9.

b Pogg. Ann., vol. 120, 1863, p. 20

c Dissertation, Jena, 1878, through Zeitschr. anal. Chemie, vol. 18, 1879, p. 99.
} 


\section{AUTHOR'S METHOD.}

a. DESCRIPTION OF THE METHOD.

In the following method there is nothing absolutely novel except that chromium and vanadium, when together, need not be separated, but are determined, the former colorimetrically, as already described (17. B, p. 147), the latter volumetrically, in the same solution. ${ }^{a}$

Five grams of the rock are thoroughly fused over the blast with 20 of sodium carbonate and 3 of sodium nitrate. After extracting with water and reducing manganese with alcohol it is probably quite unnecessary, if the fusion has been thorough, to remelt the residue as above, though for some magnetites and other ores containing larger amounts of vanadium than the generality of rocks this may be necessary, as Edo Claassen has shown. ${ }^{b}$ The aqueous extract is next nearly neutralized by nitric acid, the amount to be used having been conveniently ascertained by a blank test with exactly 20 . grams of sodium carbonate, etc., and the solution is evaporated to approximate dryness. Care should be taken to avoid overrunning neutrality, because of the reducing action of the nitrous acid set free from the nitrite produced during fusion, but when chromium is present it has been my experience that some of this will invariably be retained by the precipitated silica and alumina, though only in one case have I observed a retention of vanadium, it being then large. The use of ammonium nitrate, instead of nitric acid, for converting the sodium carbonate into nitrate does not seem to lessen the amount of chromium retained by the silica and alumina.

As a precautionary measure, therefore, and always when chromium is to be estimated also, the silica and alumina precipitate should be evaporated with hydrofluoric and sulphuric acids, the residue fused with a little sodium carbonate, and the aqueous extract again nearly neutralized with nitric acid and boiled for a few moments, the filtrate being added to the main one.

Mercurous nitrate is now added to the cold alkaline solution in some quantity, so as to obtain a precipitate of considerable bulk, con- . taining, besides mercurous carbonate, chromium, vanadium, molybdenum, tungsten, phosphorus, and arsenic, should all happen to be in the rock. The mercurous carbonate serves to counteract any acidity resulting from the decomposition of the mercurous nitrate. Precipitating in a slightly alkaline instead of a neutral solution renders the addition of precipitated mercuric oxide unnecessary for correcting this acidity. If the alkalinity, as shown by the formation of an unduly large precipitate, should have been too great, it may be reduced by

a Hillebrand, W. F., Jour. Am. Chem. Soc., vol. 20, 1898, p. 461; Chem. News, vol. 78, 1898, p. 295; Bull. U. S. Geol. Survey No. 167, 1900, p. 44.

o Am. Ohem. Jour., vol. 8, 1886, p. 437. 
careful addition of nitric acid until an added drop of mercurous nitrate no longer produces a cloud.

After heating and filtering, the precipitate is ignited in a platinum crucible after drying and removing from the paper to obviate any chance of loss of molybdenum and of injury to the crucible by reduction of arsenic. The residue is fused with a very little sodium carbonate, leached with water, and the solution, if colored yellow, filtered into a graduated flask of $25 \mathrm{~cm}^{3}$ or more capacity. The chromium is then determined accurately in a few minutes by comparing with a standard alkaline solution of potassium monochromate (17. B, p. 147). Then, or earlier in absence of chromium, sulphuric acid is added in slight excess, and molybdenum and arsenic, together with occasional traces of platinum, are precipitated by hydrogen sulphide, preferably in a small pressure bottle. ${ }^{a}$ If the color of the precipitate indicates absence of arsenic the filter with its contents is carefully ignited in porcelain, and the delicate sulphuric-acid test for molybdenum is applied as follows: The molybdenum compound is heated in porcelain with a single drop of strong sulphuric acid till the acid is nearly volatilized. On cooling a beautiful blue color is proof of the presence of molybdenum.

The filtrate, in bulk from $25 \mathrm{~cm}^{3}$ to $100 \mathrm{~cm} .^{3}$, is boiled to expel hydrogen sulphide, and titrated at a temperature of $70^{\circ}$ to $80^{\circ}$ with a very dilute solution of permanganate, representing about $1 \mathrm{mg}$. of $\mathrm{V}_{2} \mathrm{O}_{5}$ per cubic centimeter, as calculated from the iron strength of the permanganate, one molecule of $\mathrm{V}_{2} \mathrm{O}_{5}$ being indicated for each one of $\mathrm{Fe}_{2} \mathrm{O}_{3}$. One or two checks are always to be made by reducing again, by means of a current of sulphur-dioxide gas, boiling this out again, ${ }^{b}$ and repeating the titration. The latter results are apt to be a very little lower than the first and are to be taken as the correct ones

\section{b. CONFIRMATORY QUALITATIVE TESTS.}

In case the volume of permanganate used is so small as to make doubtful the presence of vanadium, it is necessary to apply a qualitative test, which is best made as follows: The solution is evaporated and heated to expel excess of sulphuric acid, the residue is taken up with 2 or $3 \mathrm{~cm} .{ }^{3}$ of water and a few drops of dilute nitric acid, and a couple of drops of hydrogen peroxide are added. A characteristic

\footnotetext{
a From a sulphuric solution the separation of platinum and molybdenum by hydrogen sulphide is much more rapid and satisfactory than from a hydrochloric solution.

$b$ 'The direct use of a solution of sulphur dioxide or of an alkali sulphite is inadmissible unless these have been freshly prepared, since after a lapse of time they contain other oxidizable bodies than sulphurous acid or a sulphite. The sulphur dioxide is best obtained as wanted by heating a flask containing a solution of sulphur dioxide, or of a sulphite to which sulphuric acid has been added.

The expulsion of the last traces of sulphur dioxide is said to be more effectively accomplished by boiling with simultaneous passage of a rapid current of carbon dioxide for a few minutes at the last than by boiling alone. Because of the small amount of air carried with it, long passage of the gas is said to result in slight oxidation of the vanadium (Manasse, O., Ann. Chem. u. Pharm., vol. 240, 1887, p. 23; Zeitschr. anal. Chemie vol. 32,1893, p. 225).
} 
brownish tint indicates vanadium. Unless the greater part of the free sulphuric acid has been removed the appearance of this color is sometimes not immediate and pronounced, hence the above precaution. It is also necessary that the nitric acid shall be in considerable excess, since in a neutral or only faintly acid solution the color does not appear strongly.

The above is a surer test to apply than the following: Reduce the bulk to about $10 \mathrm{~cm} .^{3}$, add ammonia in excess, and introduce hydrogen sulphide to saturation. The beautiful cherry-red color of vanadium in ammonium-sulphide solution is much more intense than that caused by hydrogen peroxide in acid solution, but the action of ammonia is to precipitate part or all of the vanadium with the chromium or aluminum that may be present or with the manganese used in titrating, and ammonium sulphide is unable to extract the vanadium wholly from these combinations. Usually, however, the solution will show some coloration, and addition of an acid precipitates brown vanadium sulphide, which can be collected, ignited, and further tested if desired.

c. APPLICATION OF THE METHOD IN PRESENCE OF RELATIVELY MUCH CHROMIUM.

The application of the method in its foregoing simplest form is subject to one limitation - the chromium must not be present above a certain moderate amount. This limitation is due to the considerable amount of permanganate then required to produce a clear transition tint when titrating in a hot solution, as is advisable with vanadium. In a cold solution of chromic sulphate much less permanganate is needed to produce the peculiar blackish tint without a shade of green, which affords a sure indication of excess of permanganate, but in a hot and especially a boiling solution the oxidation of the chromium itself takes place so rapidly that a very large excess of the reagent may be added before a pronounced end reaction is obtained. Nevertheless, fairly satisfactory determinations of as little as 1 or $2 \mathrm{mg}$. of vanadium pentoxide can be made in presence of as much as 30 $\mathrm{mg}$. of chromic oxide. To accomplish this it is only necessary to apply a simple correction obtained by adding permanganate to a like bulk of equally hot chromic sulphate solution containing approximately the same amount of chromium.

C. H. Ridsdale ${ }^{a}$ titrated the cold solution to avoid oxidation of chromium and obtained accurate results, but in my experience the end reaction is then uncertain.

The following tables contain the results of a considerable number of tests, those in Table 2 being tabulated separately in order to show the degree of accuracy attainable with a large excess of chromium by 
applying the correction mentioned above and also the amount of this correction:

TABLE 1.-Tests for vanadium in the presence of chromium.

\begin{tabular}{|c|c|c|c|c|c|c|c|c|c|}
\hline No. & $\begin{array}{l}\text { Chromic } \\
\text { oxide. }\end{array}$ & $\begin{array}{l}\text { Vanadium } \\
\text { pentoxide. }\end{array}$ & $\begin{array}{l}\text { Vanadium } \\
\text { pentoxide } \\
\text { found. }\end{array}$ & Error. & No. & $\begin{array}{c}\text { Chromic } \\
\text { oxide. }\end{array}$ & $\begin{array}{l}\text { Vanadium } \\
\text { pentoxide. }\end{array}$ & $\begin{array}{l}\text { Vanadium } \\
\text { pentoxide } \\
\text { found. }\end{array}$ & Error. \\
\hline & Milligrams & Milligrams & Milligrams & Milligram & & Milligrams & Milligrams & Milligrams & Milligram \\
\hline 1 & 1 & 9.37 & 9.22 & -0.15 & 7 & 3.5 & 18.74 & 18.97 & $\begin{array}{r}0.23 \\
+0.23\end{array}$ \\
\hline 2 & 1 & .94 & 1.04 & +.10 & 8 & 6 & 5.6 & .0 .1 & +.50 \\
\hline 3 & 1.5 & 5.25 & $\begin{array}{r}.98 \\
5.49\end{array}$ & $\begin{array}{l}+.04 \\
+.24\end{array}$ & $\begin{array}{r}9 \\
10\end{array}$ & $\begin{array}{l}6 \\
6\end{array}$ & $\begin{array}{l}4.6 \\
5.6\end{array}$ & $\begin{array}{l}4.78 \\
5.58\end{array}$ & $\begin{array}{r}+.10 \\
-\quad .04\end{array}$ \\
\hline & & & 5. 43 & +.19 & 11 & 10 & 5.6 & 5. 58 & -.04 \\
\hline 4 & 2 & 5. 62 & 5.5 & -.12 & 1.2 & 10 & 23.52 & 23.81 & +.29 \\
\hline 5 & 3 & 4. 68 & 4.78 & $\begin{array}{r}+12 \\
+.10\end{array}$ & 13 & 10 & 46.85 & 46.98 & $\begin{array}{r}+.19 \\
+.13\end{array}$ \\
\hline & & & 4.78 & +.10 & & & & & $\circ+.35$ \\
\hline & & & 4. 83 & +.15 & 14 & 25 & 23.52 & 23.65 & +.13 \\
\hline 6 & 3 & 5. 62 & & -.04 & & & & & \\
\hline 7 & 3.5 & 18.74 & 18.89 & +.15 & 10 & 81.5 & 23.02 & 23.71 & +.19 \\
\hline
\end{tabular}

TABLE 2.-Application of correction for larger amounts of chromium, obtained by adding potassium permanganate to an equal bulk of solution containing a like amount of chromic sulphate.

\begin{tabular}{|c|c|c|c|c|c|c|}
\hline \multirow{2}{*}{ No. } & \multirow{2}{*}{$\begin{array}{l}\text { Chromic } \\
\text { oxide. }\end{array}$} & \multirow{2}{*}{$\begin{array}{l}\text { Vanadium } \\
\text { pentoxide. }\end{array}$} & \multicolumn{2}{|c|}{$\begin{array}{l}\text { Vanadium pentoxicle } \\
\text { found. }\end{array}$} & \multirow{2}{*}{ Frror. } & \multirow{2}{*}{$\begin{array}{c}\text { Volnme of soln } \\
\text { tion. }\end{array}$} \\
\hline & & & $\begin{array}{l}\text { Uncor- } \\
\text { rected. }\end{array}$ & Corrected & & \\
\hline & Milligrams. & Milligrams. & Milligrams. & Milligrams. & Milligram. & \\
\hline 16 & 20 & $\begin{array}{r}0.94 \\
\end{array}$ & 1.59 & $\begin{array}{r}0.99 \\
0.09\end{array}$ & $\begin{array}{r}+0.05 \\
+\end{array}$ & 50 to $100 \mathrm{~cm}{ }^{8}$ \\
\hline & & 1.87 & $\begin{array}{r}2.69 \\
2.39 \\
2.59\end{array}$ & $\begin{array}{l}2.09 \\
1.79 \\
1.99\end{array}$ & $\begin{array}{r}+.22 \\
-.08\end{array}$ & 50 to $100 \mathrm{~cm} .^{3}$ \\
\hline 18 & 20 & 18.74 & 19.4 & 18. 73 & -.01 & 50 to $100 \mathrm{~cm}^{3}$ \\
\hline & & & $\begin{array}{l}19.3 \\
19.3\end{array}$ & $\begin{array}{l}18.63 \\
18.63\end{array}$ & $=.11$ & \\
\hline 19 & 30 & 1.87 & 2.99 & 2.14 & +.27 & A bout $100 \mathrm{~cm}^{3}$ \\
\hline & & & $\begin{array}{l}2.79 \\
2.79\end{array}$ & $\begin{array}{l}1.94 \\
1.94\end{array}$ & $\begin{array}{l}+.07 \\
+.07\end{array}$ & \\
\hline & & & 2. 69 & 1.84 & -.03 & \\
\hline & & & 2.69 & 1.84 & -.03 & \\
\hline 20 & 30 & 1.87 & $\begin{array}{l}2.69 \\
2.89\end{array}$ & $\begin{array}{l}1.79 \\
2.09\end{array}$ & $\begin{array}{l}-.08 \\
+.22\end{array}$ & $200 \mathrm{~cm}^{3}$ \\
\hline & & & 2.89 & 2.09 & +.22 & \\
\hline 21 & & & 2.79 & 1.99 & +.12 & 000 \\
\hline 21 & 62 & 46.85 & 48.60 & 47.60 & +.75 & $200 \mathrm{~cm}^{3}$ \\
\hline
\end{tabular}

In spite of the fact that the correction in most of the trials of this last table represents a large proportion of the permanganate used, the results must be considered satisfactory in view of the small amount of vanadium present, and they show that the method in competent hands after a little experience affords trustworthy figures.

The method of T. Fischer ${ }^{a}$-digestion of the precipitated lead salts with a strong solution of potassium carbonate-appears to offer the long-needed satisfactory quantitative separation of arsenic, phosphorus, chromium, tungsten, and molybdenum from vanadium, the normal lead metavanadate remaining quite unattacked, according to 
Fischer, while the other lead salts are wholly decomposed, but the applicability of this method to the separation of the minute amounts often found in rocks and ores has not been tested. The object has been in the present case to reach satisfactory results with the greatest expedition, and when chromium is not present in considerable amount this is accomplished.

Fortunately, chromium is almost never a prominent constituent of clays, coals, iron ores, and those rocks in which vanadium has thus far been reported, for although it is usually certain of the most basic of the silicate rocks that are highest in chromium - as the peridotitesyet in these, so far as present experience teaches, vanadium is lacking, a fact doubtless connected with the simultaneous absence from them of ferric aluminous silicates.

\section{COLORIMETRIC METHOD FOR VANADIUM.}

In the paper already cited ${ }^{a}$ the possibility of a colorimetric method for vanadium, based on its behavior toward hydrogen peroxide, was suggested. L. Maillard ${ }^{b}$ has since developed such a method, though it has not been tested in the Survey laboratory.

\section{FERROUS IRON.}

A. OXIDATION OF FERROUS IRON IN MINERALS BY GRINDING-PREPARATION OF SAMPLE.

As already pointed out under 3. C (p. 52), the effect of grinding in air is to lower the $\mathrm{FeO}$ and increase the $\mathrm{Fe}_{2} \mathrm{O}_{3}$ in a mineral powder that contains $\mathrm{FeO}{ }^{c}$ Of the latter 20 to 30 per cent or more may thus be caused to disappear by grinding steadily for two hours. Attempts to prevent this chemical change by grinding under water; alcohol, and carbon tetrachloride have been partly successful, but not enough so to permit the substitution in general practice of one of these media for air. Grinding in a wholly inert atmosphere, such as nitrogen (not carbon dioxide, which I find seems to form carbonates) might be successful in preventing oxidation in large part if not wholly, but is quite impracticable for general work. That it would not prevent taking up both loosely and firmly held water on subsequent contact of the powder with the air is certain, as such absorption is due to increased surface exposure. From the fact that oxidation is greatly lessened by grinding under one of the abovenamed liquids, even though the powder be then exposed to air, it follows that increased surface exposure is not the dominant factor

\footnotetext{
a Hillebrand, W. F., Jour. Am. Chem. Soc., vol. 20, 1898, p. 461; Chem. News, vol. 78, 1898, p. 295; Bull: U. S. Geol. Survey No. 167, 1900, p. 44.

$b$ Bull. Soc. chim., vol. 23, 1900, p. 559.

c Cf. Mauzelius, R., Sveriges Geol. Undersökning, Arsbok I, No. 3, 1907; Hillebrand, W. F., Jour. Am. Chem. Soc., vol. 30, 1908, p. 1120; and Chem. News, vol. 98, 1908, pp. 205, 215.
} 
in promoting oxidation, but probably local heating as the grains are fractured and rubbed under the pestle. The effect seems to be influenced also by the hardness of the iron minerals and those associated with them, and perhaps still more by the chemical nature of the iron-bearing molecules themselves.

The following table contains some selected and typical data, taken from the paper already cited, ${ }^{a}$ that show the effect on the $\mathrm{FeO}$ content of grinding different rocks under different conditions and also the comparative merits of the Pratt and Cooke methods of determining ferrous iron:

Effect of grinding on the ferrous-iron content of rock powders.

\begin{tabular}{|c|c|c|c|c|c|c|}
\hline \multirow{2}{*}{$\begin{array}{l}\text { Material and maximum } \\
\text { size of grain of bulk } \\
\text { sample. }\end{array}$} & \multicolumn{4}{|c|}{ Conditions of grinding. } & \multicolumn{2}{|c|}{$\mathrm{FeO}$ (per cent). } \\
\hline & No. & Grams. & $\begin{array}{l}\text { Time } \\
\text { (min- } \\
\text { utes). }\end{array}$ & Medium. & Pratt method. & $\begin{array}{l}\text { Cooke } \\
\text { method. }\end{array}$ \\
\hline Basilt, $0.08 \mathrm{~mm} . . . \ldots \ldots \ldots$ & $\begin{array}{l}1 \mathrm{a} \\
1 \mathrm{~b} \\
1 \mathrm{c}\end{array}$ & $\begin{array}{r}\text { Unground. } \\
4 \\
4\end{array}$ & $\begin{array}{r}300 \\
30 \\
30\end{array}$ & $\begin{array}{l}\text { Abs. alc. } \\
\text { Air. }\end{array}$ & $\begin{array}{r}7.03,7.07,7.09,7.11 \\
7.09,7.12,7.14 \\
7.01,7.07,7.08,7.16 \\
7.18,7.23\end{array}$ & \\
\hline Diabasic norite or gabbro.. & $\begin{array}{l}1 \mathrm{~d} \\
2 \mathrm{a} \\
2 \mathrm{~b} \\
2 \mathrm{c} \\
2 \mathrm{~d} \\
2 \mathrm{o} \\
3 \mathrm{a}\end{array}$ & $\begin{array}{r}4 \\
\text { Unground. } \\
4 \\
4 \\
4 \\
4 \\
\text { Unground. }\end{array}$ & $\begin{array}{l}120 \\
120 \\
120 \\
140 \\
120\end{array}$ & \begin{tabular}{r} 
Air. \\
\hdashline$\ldots \ldots$ abs. alc. \\
CCls. \\
$\mathrm{H}_{2} \mathrm{O}$. \\
Air.
\end{tabular} & $\begin{array}{r}6.84,6.90 \\
3.65,3.65 \\
3.47,3.49 \\
3.30,3.37 \\
3.40,3.43 \\
\text { 3. } 28,3.36,3.40 \\
13.55,13.35\end{array}$ & $\begin{array}{r}\text { 3. } 55,3.52 \\
\text { 3. } 40,3.49 \\
\text { 3. } 25,3.30 \\
\text { 3. } 36,3.38 \\
\text { 3. } 29,3.38 \\
13.54\end{array}$ \\
\hline & $\begin{array}{r}3 \mathrm{~b} \\
3 \mathrm{c} \\
3 \mathrm{~d}\end{array}$ & $\begin{array}{l}4 \\
4 \\
4\end{array}$ & $\begin{array}{l}20 \\
60 \\
30\end{array}$ & $\begin{array}{l}\text { Abs. alc. } \\
\qquad \begin{array}{l}\mathrm{CCl}_{4} . \\
\mathrm{Air} .\end{array}\end{array}$ & $\begin{array}{r}13.00,13.10,13.11 \\
13.16,13.19 \\
12.88,12.93 \\
12.91,12.93,13.06,\end{array}$ & $\begin{array}{r}13.11 \\
13.06,13.07\end{array}$ \\
\hline Andesite, $0.08 \mathrm{~mm} . . . \ldots .$. & $\begin{array}{l}3 \mathrm{e} \\
4 \mathrm{a} \\
4 \mathrm{~b}\end{array}$ & $\begin{array}{r}4 \\
\text { Unground. }\end{array}$ & $\begin{array}{l}120 \\
\cdots 0\end{array}$ & Air. & $\begin{array}{r}12.59,12.66 \\
3.32,3.34\end{array}$ & \\
\hline $\begin{array}{l}\text { Altered basaltic green- } \\
\text { stone, } 0.18 \mathrm{~mm} \text {. }\end{array}$ & $\begin{array}{l}40 \\
5 \mathrm{a}\end{array}$ & Unground. & & Alr. & $\begin{array}{r}3.09 \\
6.68\end{array}$ & \\
\hline Quartz diorite, $0.11 \mathrm{~mm} .$. & $\begin{array}{l}5 b \\
6 a\end{array}$ & Unground. & 120 & Air. & $\begin{array}{r}\text { 3. } 64 \\
2.58\end{array}$ & \\
\hline & $\begin{array}{l}6 \mathrm{~b} \\
7 \mathrm{a}\end{array}$ & Unground. & 120 & Air. & $\begin{array}{r}1.67 \\
2.08,2.09\end{array}$ & \\
\hline & $\begin{array}{l}7 b \\
7 c\end{array}$ & & $\begin{array}{r}120 \\
30\end{array}$ & $\begin{array}{l}\text { Abs. alc. } \\
\text { Air. }\end{array}$ & $\begin{array}{l}\text { 2. } 01,2.01 \\
\text { 2. } 04,2.07\end{array}$ & \\
\hline Altered basaltic rock. 0.18 & $\begin{array}{l}7 \mathrm{~d} \\
8 \mathrm{a}\end{array}$ & Unground. & 120 & Air. & $\begin{array}{l}\text { 1. } 47,1.47 \\
8.33,8.34\end{array}$ & \\
\hline min. & $\begin{array}{l}8 b \\
8 c \\
8 d\end{array}$ & $\begin{array}{r}4 \\
4 \\
\cdot(?)^{4}\end{array}$ & $\begin{array}{r}120 \\
30 \\
(?)\end{array}$ & $\begin{array}{l}\text { A bs. alc. } \\
\text { Air. } \\
\text { Air. }\end{array}$ & $\begin{array}{r}8.11,8.14 \\
8.23 \\
5.74\end{array}$ & \\
\hline
\end{tabular}

The experiments carried out by me show several things: (1) Although comparatively brief grinding (fifteen to thirty minutes) in air and long grinding under alcohol does not, as a rule, yield powders that are markedly oxidized (sometimes almost no oxidation seems to have taken place), this is not always so; hence grinding under one or the other of these media can not be recommended as a practice to follow at.all times. The data for short and long grinding in the same enveloping medium are not strictly comparable, as the former was done by hand, the latter by a more effective mechanical process. (2) Alcohol, in spite of its greater solvent power for oxygen, seems 
to be a little more effective in preventing oxidation than water. It has the further advantage that it can be more quickly removed afterwards. (3) Of the organic media employed alcohol seems to be more efficacious than carbon tetrachloride. (4) The effect of the presence of refractory iron-bearing minerals (garnet and others) is indicated by the rather wide variations in duplicate determinations, as shown under $i$ and 3 of the table, when the material is not in a fine state of division. (6). The agreement that can be expected in duplicate determinations by either the Pratt or the Cooke method is excellent with fine powders or with coarse powders that yield. readily to attack by hydrofluoric acid. (6) Inasmuch as powders so fine as to pass sieves of 30 and even 60 meshes to the linear centimeter often show much less than 0.1 per cent of moisture if hydrous alteration products are absent, it is reasonable to conclude that in their preparation there has been occasioned relatively as little oxidation of ferrous iron as absorption of moisture, on the assumption that the small amount of water found results from the increased surface exposure due to crushing and is not inherent. Therefore, if the powder is completely decomposed by hydrofluoric acid it is safe to assume that a determination of ferrous iron in it, carried out with proper precautions, will give a result close to the truth when other disturbing factors are not in evidence. (7) With different minerals very different degrees of oxidation are brought about under like conditions, from a few per cent (1d) up to about 45 per cent (5b) of the $\mathrm{FeO}$ after several hours' grinding, and it is not always the mineral that might a priori be regarded as most oxidizable that shows most oxidation. (See paper cited.) Further, a soft or tough mineral undergoes greater oxidation if ground with a hard one than by itself alone.

It is plain that nearly all mineral analyses which have been made in the past are affected by more or less serious errors in respect not only to the oxides of iron but also to water, the error being greater the further the comminution of the sample was carried. Very many analyses show ferric iron in minerals that should apparently contain none and small amounts of water for which there seems to be no place in any probable formula. These are discordances for which a simple and in most cases probable explanation is now offered. A revision of much past analytical work, particularly that relating to iron minerals, is called for, but before it can be properly done adequate provision must be made' to avoid the old errors. In the paragraphs following are set forth the procedures that seem best adapted to attain this end in the light of present experience. Much routine and experimental work, must, however, yet be done before rules to fit all cases can be formulated. It is perhaps advisable to add here, in order to prevent misconception, that the errors in past work are 
not as a rule to be assumed equal to those in the above table that resulted from grinding for two hours or more, for few if any analysts have made it a rule to grind for such a length of time.

The mineral powder that has been prepared by crushing without grinding (3. B, p. 50) serves as the starting point. If trial shows that gentle boiling of it for twenty minutes with hydrofluoric acid leaves no residue or but little, this sample is to be used directly for the ferrous iron determination. If the residue is considerable, a weighed portion ( 0.5 to $1.0 \mathrm{gram}$ ) of the sample is to be ground with absolute alcohol in a large agate mortar only long enough to yield a powder that will leave little or no residue. The alcohol is then to be allowed to evaporate spontaneously, and when the last trace has disappeared the powder is to be transferred to a platinum crucible of 80 to $100 \mathrm{~cm} \cdot{ }^{3}$ capacity, the powder which may adhere to the mortar and pestle being also transferred by the aid of a fine jet of hot water. The subsequent operations are given under the respective methods (D, below), which are those involving decomposition by hydrofluoric acid. The Mitscherlich method (C, below) requires in almost every case a very fine powder. For this and other reasons that method finds little application. For the same reason the modifications of the hydrofluoric acid method that require a powder of considerable fineness (D. d. $\beta, \gamma$ ) will be less applied than the one (D. d. $\alpha$ ) which permits the use of a relatively coarse powder. If a very fine powder must be employed there seems to be no way known at present of correcting for whatever oxidation may have taken place during grinding. It will be preferable in such cases to grind under alcohol a small weighed portion of the coarse sample, instead of grinding a large portion and taking from this an aliquot part, the reason being that in the latter case separate determinations of both loosely and firmly held water must be made in order to be able to correct the $\mathrm{FeO}$ found for the considerable weight of water taken up during grinding (5. B, p. 64).

\section{B. COMPARISON OF SEALED-TUBE AND HYDROFLUORIC ACID METHODS-} COMPARATIVE WORTHLESSNESS OF THE FORMER IN ROCK ANALYSIS.

No point in rock analysis has been the cause of greater solicitude to the chemist, and especially to the mineralogist and petrographer, than the determination of iron in ferrous condition. The sealed-tube or Mitscherlich method with sulphuric acid, for a long time the only available one, is in theory perfect, since complete exclusion of oxygen is easily attainable. Its earliest recognized defect lies in the inability to secure always complete decomposition of the iron-bearing minerals, and even to ascertain, oftentimes, whether or not the decomposition has been complete. The addition of hydrofluoric acid to the sulphuric in the tube, in order to insure this breaking up, is to be regarded 
as of very doubtful utility in most cases, since the glass may be so strongly attacked as to add an appreciable amount of iron to the solution, and the hydrofluoric acid may have exhausted itself in attacking the glass before the more refractory minerals succumb. Nevertheless, if decomposition can be effectęd by sulphuric acid alone the results obtained are sharp and concordant; but they are in rock analysis usually higher than when made by any of the modifications of the hydrofluoric-acid method now so extensively practiced. This difference is not very marked with rocks containing but 1 or 2 per cent of ferrous iron, but it increases with rising percentage to such an extent that where the sealed-tube method will show 12 per cent ferrous oxide the other may indicate no more than 10 per cent. This is a fact of which I have long been cognizant, but it seems not to have been known to chemists or petrographers at large, though $\mathrm{E}$. A. Wülfing ${ }^{a}$ noticed this difference in certain analyses without appreciating its significance. Experiments with soluble iron salts of known composition, like ferrous sulphate and ferrous-ammonium sulphate, throw no light on the subject, for both methods give with them the same sharp and accurate results.

The key to the problem was discovered by L. L. de Koninck ${ }^{b}$ a good many years ago, but remained unknown to the chemical world by reason of its obscure medium of publication until rediscovered by Dr. H. N. Stokes in this laboratory, during an investigation on the action of ferric salts on pyrite and other sulphides. ${ }^{c}$ That oxidation of the sulphide and reduction of the ferric salt hereby takes place was recognized by J. H. L. Vogt, ${ }^{d}$ but not the ease with which the change takes place and the completeness of the oxidation of the pyrite, not only of its iron but of the greater part of the sulphur as well. Pure pyrite itself is attacked with extreme slowness by boiling dilute sulphuric and hydrofluoric acids, either alone or mixed, but the moment a ferric salt is introduced the case is altogether different.

The complete solution to the problem was afforded by the observation made in the Survey laboratory that rocks with hardly an exception and many minerals carry pyrite or pyrrhotite, or both, often in considerable amount, often in traces only. Sulphur can almost always be detected in 2 grams of rock powder.

Experiment has shown (D. c. $\alpha$, p. 165) that with the amounts of sulphides usually found in igneous rocks their effect on the estimation of ferrous iron by the hydrofluoric-acid method at atmospheric pressure and boiling heat is negligible, though by increasing the amount of sulphide the effect becomes more and more apparent:

a Ber. Deutsch. chem. Gesell., vol. 32, 1899, p. 2217, footnote.

${ }^{b}$ Ann. Soc. géol. Belgique, vol. 10; 1882-83, p. 101; Zeitschr. anorg. Chemie, vol. 26, 1901, p. 123.

$c$ Bull. U. S. Geol. Survey No. 186, 1901; Am. Jour. Sci., 4th ser., vol. 12, 1901, p. 414.

d Zeitschr. prakt. .Geol., 1899, pp. 250-251. 
because of the greater surface of pyrite exposed to the action of the ferric iron of the rock.

Under the conditions of the Mitscherlich method, on the other hand-a temperature of 150 to $200^{\circ}$, and even higher, high pressure, much longer time of action, and impossibility of escape of any hydrogen sulphide that may be formed-the sulphur of the sulphides becomes nearly if not fully oxidized to sulphuric acid at the expense of the ferric iron in the rock, with the production of an equivalent amount of ferrous iron in addition to that resulting from the sulphide itself.

Let us now see what the effect of these traces of sulphides when fully oxidized amounts to. One atom of sulphur (32) requires for its complete conversion to trioxide the oxygen of three molecules of ferric oxide (480), which then becomes six molecules of ferrous oxide (4:32). In other words, 0.01 per cent of sulphur may cause the ferrous oxide to appear too high by 0.135 per cent, and 0.10 per cent of sulphur may bring about an error of 1.35 per cent in ferrous oxide. The case is still worse if the sulphur is set free as hydrogen sulphide from a soluble sulphide, for then the above percentages of sulphur produce errors of 0.18 and 1.8 per cent, respectively, in the ferrous oxide determination.

The error caused by sulphides tends to become greater the more there is present of either or both sulphide and ferric salt. . Now, the highly ferruginous rocks usually carry more ferric iron than the less ferruginous ones, and they are often relatively high in pyrite and pyrrhotite; hence the increasing discrepancy between the results by the two methods as the iron contents of the rocks rise is fully in accord with the above explanation. ${ }^{a}$

Of course carbonaceous matter will, under the conditions of the Mitscherlich method, likewise reduce sulphuric acid and cause the determination of ferrous iron to be faulty.

Notwithstanding the fact that the Mitscherlich method has thus been discredited in its general applicability to rocks and minerals, it is still probably the best with those which are totally free from sulphides and wholly decomposable. Hence the conditions under which success can best be achieved by it are set forth in the following paragraphs.

\section{THE MODIFIED MITSCHERLICH METHOD.}

$\alpha$. Strength of acid.-The method in its original and usual application calls for a mixture of 3 parts of sulphuric acid and 1 of water by weight, or about 3 to 2 by volume, though a still stronger acid is some-

a For details of experiments showing the worthlessness of the Mitscherlich method for rocks and minerals which contain even a trace of free sulphur or sulphides, see Hillebrand, W. F., and Stokes, H. N., Relative value of the Mitscherlich and hydrofluoric acid methods for ferrous-iron determinations: Jour. Am. Chem. Soc., vol. 22, 1900, p. 625; and Zeitschr. anorg. Chemie, vol. 25, 1900, p. 326. 
times used. In some cases, however, perhaps in most, much better decomposition of the silicates is effected by reversing the proportions of water and acid, or at any rate by diluting considerably beyond the above proportion. Hereby the separation of salts difficultly soluble in the stronger acid is avoided and the-actual solvent effect on the minerals seems to be in no wise diminished.

$\beta$. Filling, sealing, and heating of the tube.-The very finely powdered mineral having been introduced into a tube of resistant glass free from ferrous iron, the open end is drawn out, so as to leave a funnel for the introduction of the acid. A very little water is then introduced and carefully heated to boiling for a moment to expel all air from the powder. The diluted acid-which has just been boiled down from a state of greater dilution in order to have it free from airis then poured in until the tube is about three-fourths filled. Carbon dioxide is then introduced, from a generator which has been in active operation for some time, through a narrow glass tube drawn out of the same kind of glass as that of which the decomposing tube consists. In a few moments the air is expelled, and the small tube is then sealed into the large one over the blast lamp without interrupting the gas current until the very last instant, when to prolong it would perhaps cause a blowing out of the softened glass. 'The interruption of the current at the proper moment is easily effected by the pressure of the thumb and finger holding the small tube at the point where it enters the rubber tube leading from the gas generator. No breakage in the oven ever occurs as a consequence of thus fusing one tube into the other.

The heating is done in a bomb oven at any desired temperature up to, say, $200^{\circ}$, and continued at intervals until examination by aid of a low-power lens shows that decomposition is complete or has progressed as far as can be hoped for. By inclosing the glass in an outer tube of strong steel, properly capped ${ }^{a}$ and containing a little ether or benzine to equalize the pressure on both sides of the glass, the temperature can be elevated far beyond what is otherwise permissible, and the decomposition will then doubtless be more complete with refractory silicates.

$r$. Reason for introducing gas and sealing as above directed.-The usual practice in employing the above method has been to expel air before sealing by introducing a few crystals or lumps of an alkali carbonate or bicarbonate, the gas set free on their contact with the acid being supposed to effectively expel all air. That this is not accomplished the following series of comparative results, long since published elsewhere, ${ }^{b}$ fully shows. The material used was the oxide of

a UUllmann, C., Zeitschr. angew. Chemie, 1893, p. 274; Zeitschr. anal. Chemie, vol. 33, 1894, p. 582.

$b$ Bull. U. S. Geol. Survey No. 78, 1891, p. 50; Chem. News, vol. 64, 1891, p. 232. 
uranium $\mathrm{U}_{3} \mathrm{O}_{8}$, requiring by theory 32.07 per cent of $\mathrm{UO}_{2}$. Operating as just described on from 0.3 to 0.5 gram, the results were

$$
31.06,31.07,29.72,29.33,29.89,30.69 \text {, }
$$

whereas after filling the tube with gas from a generator there was found

$$
32.11,31.90,32.15,32.12,32.06,32.17,32.28 \text {, }
$$

the average error of the former series being 1.78 per cent. The percentage error would, of course, be reduced by increasing the weight of mineral operated on. An average error equal to the above when employing 1 gram of ferrous minerals would make the percentage for $\mathrm{FeO}$ about 0.3 per cent too low. While the absolute error might be the same in all cases, the relative error would increase with minerals low in ferrous iron.

\section{THE HYDROFLUORIC ACID METHOD.}

a. PRINCIPLE OF THE METHOD.

This method consists simply in securing complete decomposition of the powder in absence of air by means of hydrofluoric acid in presence of sulphuric acid, and titrating the ferrous iron in the resulting solution. It is the one which has been almost exclusively used in the Survey laboratories. The form of apparatus employed until recently is the original one devised by J. P. Cooke ${ }^{a}$ (fig. 21, p. 169). This does not permit driving off the superfluous hydrofluoric acid, as does that of Treadwell (fig. 22, p. 170) to some extent, which latter ought therefore, for a reason developed below, to afford slightly lower and more accurate results, other conditions being equal. But the method as ordinarily carried out is subject to two defects of opposite sign but unequal value, the second and greater of which has remained unsuspected until recently. ${ }^{b}$

It is possible to titrate ferrous iron in presence of sulphuric acid and as much as 5 to $7 \mathrm{~cm}^{3}$ of 40 per cent hydrofluoric acid in a total volume of 200 to $400 \mathrm{~cm} \cdot{ }^{3}$ almost if not quite as exactly as in sulphuric acid alone, provided the iron solution is diluted with air-free water and the titration is made immediately after adding the hydrofluoric acid and with all possible dispatch. This condition can naturally not be fulfilled in practice, because the mineral must be in contact with the acid for a long time in the apparatus commonly employed, and it seems to be almost impossible to prevent some oxidation during this period.

a Am. Jour. Sci., 2d ser., vol. 44, 1867, p. 347.

$b$ It is not clear what, if any, bearing certain observations of E. Deussen (Monatsh. Chemie, vol. 28, 1907, p. 163) may have on the method. He claims that dissolving ferric oxide in hydrofluoric acid involves partial reduction of the iron with formation of both ferrous iron and hydrogen peroxide: $2 \mathrm{Fe}_{2} \mathrm{O}_{3}+8 \mathrm{HF}=$ $\mathrm{Fe}_{3} \mathrm{~F}_{8}+\mathrm{FeO}+3 \mathrm{H}_{2} \mathrm{O}+\mathrm{H}_{2} \mathrm{O}_{2}$. The experiments described on the following pages do not indicate such action, at least in presence of sulphuric acid.

20091-Bull. $422-10-11$ 


\section{b. DEFECTS OF THE METHOD.}

$\alpha$. Oxidizability of divalent manganese by permanganate in presence of hydrofluoric acid.-Manganic fluoride in dilute solutions is hardly at all dissociated, wherein it differs from manganic sulphate. The entering of the manganic ion as fast as formed into the undissociated state explains the fact that in presence of hydrofluoric acid divalent manganese is readily oxidized by permanganate; whereby a sharp end reaction is rendered unattainable in presence of much hydrofluoric acid, say over $7 \mathrm{~cm} .^{3}$ of 40 per cent acid. It is easy to obtain a transitory pink color throughout the liquid, but this rapidly disappears, the more rapidly the greater the amount of hydrofluoric acid or of manganous salt present. The oxidizer can be added by the cubic centimeter to solutions already containing manganous sulphate in presence of hydrofluoric acid without producing a more than passing pink color. The solution, however, takes on in ever-increasing intensity the red-brown color characteristic of manganic salts. The decolorization due to this cause is hence much more pronounced in the case of rocks high in ferrous iron than in that of those low in this constituent, because of the greater amount of manganous salt resulting from reduction of a correspondingly larger amount of permanganate, and is also greater with increasing hydrofluoric acid. In presence of but little ferrous iron, up to, say, 2 centigrams, and 5 to $7 \mathrm{~cm}^{3}$ of hydrofluoric acid the color produced by a drop of permanganate lasts some time, but is very evanescent as the ferrous iron, and consequently the manganous salt formed, increases.

E. Deussen, ${ }^{a}$ on the basis of but a few tests, ascribes the extra consumption of permanganate to some obscure action of iron and seeks to counteract it by the addition of manganous sulphate. In both these assumptions he is, however, certainly in error, as shown by the fact that the addition of a drop or two of this oxidizer to a solution of ferric sulphate in even a large amount of hydrofluoric acid produces a very stable coloration, which is destroyed by the addition of man ganous sulphate, and by the above-mentioned very evident partial reoxidation of the reduced manganese. This last, the oxidation of divalent manganese by permanganate in presence of hydrofluoric acid, can be effected with the greatest ease in entire absence of ferric iron.

Numerous tests have been made to ascertain the error due to oxidation of the manganese under conditions similar in respect to the amounts of iron involved to those encountered in rock analysis. Increasing amounts of hydrofluoric acid were added to fixed amounts of ferrous sulphate and sulphuric acid and the titration made with the least possible expenditure of time. With not more than $7 \mathrm{~cm}^{3}$ of 40 per cent hydrofluoric acid a very slight tendency was observed

a Zeitschr. anorg. Chemie, vol. 44, 1905, p. 425 . See also Monatsh. f. Chemie, vol. 28, 1907, p. 163. 
toward high results, but in most cases the results were identical with those obtained in presence of sulphuric acid alone. As the amount of hydrofluoric acid was increased to $10 \mathrm{~cm} .{ }^{3}$ a perceptible increase was noticed, which became markedly greater with $15 \mathrm{~cm}^{3}$ of the acid, amounting in this case to about $0.2 \mathrm{~cm}^{3}$ in the average on a normal consumption of $20 \mathrm{~cm}^{3}$ of permanganate of $0.0032 \mathrm{FeO}$ titer. The personal factor enters here, G. Steiger having found slightly greater differences, but it is possible that they resulted from the titrating in more concentrated solutions, for with greater dilution the differences are unquestionably less, according to experiments of my own.

Hence, after decomposition of the rock, removal of the excess of hydrofluoric acid is called for if the best results are to be expected. To a certain extent Treadwell's apparatus accomplishes this, but, as the experiments show, its complete expulsion is unnecessary. ${ }^{a}$.

$\beta$. Extreme oxidizability of divalent iron by free oxygen in presence of hydrofluoric acid or a fuoride.-In comparing two series of results obtained with the Cooke apparatus, using a solution of ferrous sulphate in sulphuric acid alone in the one and in sulphuric acid with hydrofluoric acid in the other, a minus error was in all cases observed when the latter acid was present. An observation made by'R. Peters, ${ }^{b}$ but not applied by him to the present case, explains this. Ferric fluoride, like the corresponding manganic salt, is almost undissociated in solution, whereas the sulphate undergoes considerable dissociation, and in this state counteracts, to a great extent, the tendency of any ferrous iron present to become oxidized by free oxygen. Hence the slow oxidation of divalent iron by air in sulphuric-acid solution and its very rapid oxidation in presence of hydrofluoric acid. While a sulphuric solution of ferrous sulphate exposed to the air in an open dish will hardly change in strength during an hour, a similar solution to which hydrofluoric acid has been added will suffer a large measure of oxidation in a quarter of the time. The change in titer becomes pronounced after a few minutes.

In the experiments with the Cooke apparatus just referred to, the minus error should amount to not more than 0.1 to $0.2 \mathrm{~cm}^{3}$ of permanganate on a normal consumption of $20 \mathrm{~cm}^{3}$ or 0.5 to 1 per cent of the iron, but it may be considerably greater if the utmost care is not taken to employ carbon dioxide as free as possible from air and to exclude most carefully the entrance otherwise of air into the apparatus. It will also be greater the higher the concentration, within certain limits, of the solution during titration.

a R. B. Gage (Jour. Am. Chem. Soc., vol. 31, 1909, p. 381) claims to overcome the harmful effect of free hydrofluoric acid by adding a solution of calcium phosphate immediately before titrating, thus forming calcium fluoride. His procedure in other respects follows closely that hereinafter described, and was used by him in analyzing magnetite.

b Zeitschr. phys. Chemie, vol. 26, 1898, p. 193, 
With a view to lessening the error, if possible, the mode of operating was varied by doing away with carbon dioxide, except at the start, shutting it off as soon as the bath was in active ebullition, and transferring the crucible direct from the hot bath to the titration vessel. The results were, if anything, a trifle better. As the employment of either the Cooke or the Treadwell apparatus involves long contact of the mineral with the acids, and as the experiments last mertioned were favorable in their results, it seemed as if the simple method of J. H. Pratt ${ }^{a}$ might be modified in the same sense. He avoided the use of all apparatus other than a capacious platinum crucible fitted with a perforated cover for the introduction of carbon dioxide, in which he boiled the mineral powder with sulphuric and hydrofluoric acids, the decomposition being greatly hastened by the active movement and higher temperature and usually completed in five to ten minutes. The modification consists in doing away altogether with carbon dioxide, except at the start, and depending on the steam of the boiling iron solution to exclude air, a modification which Pratt himself tried with rather considerable minus errors, resulting perhaps from his having a smaller crucible at command than the method really demands. With a crucible of $100 \mathrm{~cm} .^{3}$ capacity, the following results with ferrous sulphate show what are the possibilities of the method:

Ferrous-iron determinations by the modified Pratt method.

[Strength of permanganate $0.0032 \mathrm{FeO}$ per $\mathrm{cm}^{2}{ }^{2}$ ]

\begin{tabular}{|c|c|c|c|c|c|}
\hline $\begin{array}{l}\text { Time of } \\
\text { boiling. }\end{array}$ & $\begin{array}{l}\text { Perman- } \\
\text { ganate } \\
\text { used. }\end{array}$ & $\begin{array}{c}\text { Normal } \\
\text { consump- } \\
\text { tion of per- } \\
\text { manganate. }\end{array}$ & $\begin{array}{l}\text { Time of } \\
\text { boiling. }\end{array}$ & $\begin{array}{l}\text { Perman- } \\
\text { ganate } \\
\text { used. }\end{array}$ & $\begin{array}{c}\text { Normal } \\
\text { consump- } \\
\text { tion of per- } \\
\text { manganate. }\end{array}$ \\
\hline $\begin{array}{r}\text { Minutes. } \\
10 \\
10 \\
10 \\
10 \\
10 \\
10 \\
10 \\
10\end{array}$ & $\begin{array}{r}c m \\
4.9 \\
4.8 \\
5.0 \\
4.9 \\
9.6 \\
9.6 \\
9.6 \\
19.2\end{array}$ & $\begin{array}{r}c m .3 \\
4.8 \\
4.8 \\
4.8 \\
4.8 \\
9.6 \\
9.6 \\
9.6 \\
19.2\end{array}$ & $\begin{array}{r}\text { Minutes. } \\
10 \\
10 \\
15 \\
15 \\
15 \\
20 \\
20\end{array}$ & $\begin{array}{l}c m . .^{3} \\
19.4 \\
19.3 \\
19.25 \\
19.1 \\
19.3 \\
19.2 \\
19.3\end{array}$ & $\begin{array}{c}c m .3 \\
19.2 \\
19.2 \\
19.2 \\
19.2 \\
19.2 \\
19.2 \\
19.2\end{array}$ \\
\hline
\end{tabular}

These results leave little, if anything, to be desired. They show either normal values or a slight plus error instead of the invariable negative one of all previous determinations. The method has been tested during the last two years in comparison with that of Cooke with favorable results, a few of which are shown in the table under A, page 155. It is the method now employed by me in preference, not only because of its simplicity and quickness, but also because with it a coarser powder can be decomposed than with either the Cooke or the Treadwell method. However, since the exigencies of the $\mathrm{FeO}$ determination are now known to demand a powder as 
coarse as possible, a longer treatment has to be given than was called for by the fine powders used by Pratt.

C. INFLUENCE OF SULPHIDES, VANADIUM, AND CARBONACEOUS MATTER ON T'HE DETERMINATION OF FERROUS IRON BY THE HYDROFLUORIC ACID METHOD.

A dark color of the insoluble fluorides and silicofluorides may be due to pyrite, graphite, or carbonaceous matter. The first of these affects the result but little, the second probably not at all, and they can be distinguished by their behavior toward nitric acid. Organic matter of course renders impossible the determination of ferrous iron.

$\alpha$. Sulphides.-Pyrite, in the quantities usually met with in igneous rocks, is probably without serious effect on the ferrous-iron determination by any of the hydrofluoric acid methods. This sulphide is very resistant toward attack in the absence of oxygen, as is shown by the fact that if present in any quantity it can be readily recognized in the residue after titration. In any case it is impossible to allow for an error introduced by its possible decomposition, and the result of titration must count as ferrous iron. In the case of soluble sulphides two sources of error are introduced-that of reduction of ferric iron by hydrogen sulphide evolved, and that due to the ferrous iron which the sulphides themselves may contain, especially if pyrrhotite is present. The first of these is perhaps negligible, since most of the hydrogen sulphide would probably be expelled without reducing iron. The second is approximately measurable if it is known that pyrrhotite is the only soluble sulphide present, and its amount has been ascertained by determining the hydrogen sulphide set free on boiling with hydrochloric acid in a current of carbon dioxide. In this case a correction is to be applied to the result of titration for total ferrous iron (see also 24. C. b, p. 198, under "Sulphur").

In order to obtain quantitative data regarding the effect of pyrite on the ferrous-iron estimation by the hydrofluoric acid method the following tests were made: Part of a fine crystal of pyrite was rather finely powdered and boiled with dilute sulphuric acid, which extracted considerable ferrous iron, derived presumably from admixed or intergrown pyrrhotite, since a second boiling with fresh acid gave a negative test for ferrous iron. After washing by decantation with water, followed by alcohol and ether, the powder was dried and further pulverized. A quarter of a gram of it when treated with hydrofluoric and sulphuric acids in a large crucible by the Cooke method for ferrous iron, then rapidly filtered through a very large perforated platinum cone fitted with filter paper, required but 2 drops of a permanganate solution representing only 0.0032 gram $\mathrm{FeO}$ to the cubic centimeter.

Since, however, Dr. H. N. Stokes has found ${ }^{a}$ that the oxidizing effect of ferric salts on pyrite and other sulphides is vastly greater than 
seems to have been suspected (see p. 158), the following tests were made in order to ascertain the probable error due to this action under the conditions prevailing in rock analysis: Successive portions of 1 gram each of a hornblende schist, free from sulphur and carrying 10.09 per cent $\mathrm{FeO}$ as the mean of several determinations and 4.00 per cent $\mathrm{Fe}_{2} \mathrm{O}_{3}$, were mixed in a large $100 \mathrm{~cm} .^{3}$ platinum crucible with 0.02 , 0.025 , and 0.10 gram, respectively, of the above purified pyrite powder, and treated with hydrofluoric and sulphuric acids by the Cooke method, the water bath being at boiling heat for one hour. The cooled contents of the crucible were poured into a platinum dish containing water and titrated rapidly nearly to an end. Then, in order to get rid of the pyrite, which would obscure the end reaction by its reducing effect on the permanganate, the solution wais filtered as above and in the clear filtrate the titration was carried to completion. The results were $10.02,10.16$, and 10.70. Inasmuch as the smallest of these three charges of pyrite was several times greater than what may be considered an unusually high amount for an igneous rock, it is very evident that for all practical purposes the influence of pyrite on the ferrous determination by the Cooke method is negligible. At the same time it is to be borne in mind that with increased content in ferric iron an increased amount of pyrite will be attacked, and that the extent of this attack is influenced by the degree of fineness of the pyrite powder, which itself undergoes oxidation during grinding.

$\beta$. Vanadium.-If vanadium, when present, exists in the trivalent condition, it necessarily affects with an error varying with its amount the result of titration for ferrous iron. If the amount of vanadium is known, a correction can be applied as follows: One molecule of $\mathrm{V}_{2} \mathrm{O}_{3}$ (150.8) in oxidizing to $\mathrm{V}_{2} \mathrm{O}_{5}$ requires as much oxygen as four molecules of $\mathrm{FeO}$ (288) when oxidized to $\mathrm{Fe}_{2} \mathrm{O}_{3}$. The proportion, $150.8: 288:$ : $\mathrm{V}_{2} \mathrm{O}_{3}$ present $: x$, therefore gives the figure to be deducted from the uncorrected value for $\mathrm{FeO}$. That this correction is very needful with many of the basic rocks becomes at once evident from the following perhaps extreme example:

Found 2.50 per cent apparent $\mathrm{FeO}$ in a rock containing .13 per cent $\mathrm{V}_{2} \mathrm{O}_{3}$.

Deduct .25 per cent $\mathrm{FeO}$ equivalent in its action on $\mathrm{KMnO}_{4}$ to $.13 \mathrm{~V}_{2} \mathrm{O}_{3}$.

Leaving 2.25 per cent $\mathrm{FeO}$ corrected.

Found 5.00 per cent apparent total iron as $\mathrm{Fe}_{2} \mathrm{O}_{3}$ in the same rock.

Deduct .14 per cent $\mathrm{Fe}_{2} \mathrm{O}_{3}$ corresponding to .13 per cent $\mathrm{V}_{2} \mathrm{O}_{3}$.

Leaving 4.86 per cent corrected total iron as $\mathrm{Fe}_{2} \mathrm{O}_{3}$.

Deduct 2.50 per cent $\mathrm{Fe}_{2} \mathrm{O}_{3}$ equivalent to 2.25 per cent $\mathrm{FeO}$.

Leaving 2.36 per cent $\mathrm{Fe}_{2} \mathrm{O}_{3}$ in the rock.

Failure to correct for the vanadium in both cases would have made the figures for $\mathrm{FeO}$ and $\mathrm{Fe}_{2} \mathrm{O}_{3}$, respectively, 2.50 and 2.22 instead of 2.25 and 2.36 as shown above.

$\gamma$. Carbonaceous matter.-As said before (c, p. 165), matter of organic origin other than graphitic carbon renders the results of the ferrous-iron determination altogether unreliable. 
d. THE METHOD IN ITS VARIOUS MODIFICATIONS.

$\alpha$. According to Pratt (modified).-One-half to 1 gram of the coarsest powder ${ }^{a}$ that can be used successfully is placed in a platinum crucible of 80 to $100 \mathrm{~cm}^{3}$. capacity with a little air-free water and about $10 \mathrm{~cm} .^{3}$ of dilute sulphuric acid ( 1 acid to 3 water by volume). If the rock contains carbonates there will be effervescence, hence the acid must be added cautiously and the cover placed on till the action is over. There need be no fear of oxidation of ferrous iron at this stage, should any go into solution. Air-free hot water is now added till the crucible is at least half full; the crucible with cover on is placed on a triangle well down over a lamp turned low and protected from drafts. The air in the crucible is displaced rapidly by carbon dioxide entering beneath the lid slightly raised on one side. In a few seconds the liquid boils, but before allowing this to happen the gas current is stopped and the well-fitting lid lowered. Then from a small platinum crucible a measured quantity of strong hydrofluoric acid $\left(5\right.$ to $\left.7 \mathrm{~cm} .^{3}\right)$ is poured in with one hand, while the other draws the lid a little to one side. A second of time suffices. The lid is replaced, the flame is increased for a few moments with great caution not to allow boiling over, and the moment steam is seen to issue around the lid it is again lowered to a point that causes steady ebullition without danger of loss. Steam should issue continually for five, ten, or as many minutes as may be deemed necessary or allowable. Then the crucible, still covered, is transferred with the tongs shown in fig. 1 (p. 30) to the titration dish, containing. cold freshly boiled water (usually about $300 \mathrm{~cm}^{3}$ ). and additional sulphuric acid, which is already under the burette, the contents of the crucible are quickly poured in with the rinsings, and the permanganate is run in as rapidly as possible with constant but gentle stirring till the first pink blush appears throughout the whole liquid for at least a couple of seconds. With little iron the color will last some time, but with increasing amounts, as also with increasing. amounts of hydrofluoric acid, it fades out with ever greater rapidity. In making a duplicate it is well to run in a little less than the full amount of permanganate before introducing the iron solution.

The method should first be tested by the novice with ferrous-sulphate solution that has been standardized without hydrofluoric acid. It is important not to prolong the boiling unduly, for salts not easily soluble will separate, and as the temperature rises the oxidizing action of the concentrating sulphuric acid comes into play.

If an unattacked residue shows after titrating, this should be allowed to settle completely, then freed from the liquid by a decantation with water, once repeated. It is now transferred by a jet of water to a

$a$ The fineness of grinding will depend on the nature of the rock. Most granitic rocks require but moderately fine grinding, those high in ferruginous and refractory minerals generally, as tourmaline, need to be very fine. 
small agate mortar, allowed to settle again, freed from most of the water by decantation, ground for a few minutes under the water that remains, and washed back into the large crucible. The treatment with hydrofluoric and sulphuric acids, in smaller amounts and for a shorter time than at first, is then to be repeated. The succeeding titration can be made in the crucible itself after quickly filling this nearly full of cold air-free water. Usually decomposition will now be complete; if not, the various operations are to be repeated. In the very short time required for grinding the residues under water no appreciable oxidation of ferrous iron need be feared.

The directions given by $\operatorname{Pratt}^{a}$ with reference to the treatment of a possible undissolved residue of very refractory minerals must be understood as applying only to homogeneous minerals and not to rocks, where the relations of ferrous and ferric iron in the undecomposed portion are certainly different from those in the part dissolved.

- 3 . According to Cooke. ${ }^{b}$-The apparatus, as shown in fig. 21 , consists of a small water bath of a single opening and covered with a glass funnel the stem of which has been cut off near the flare, resting in a troughlike depression of the specially made cover. Into this trough water constantly drops from a tubulated bottle, thus securing a perfect water joint and serving to keep the bath full by overflowing on the inside. For the more perfect exclusion of air it is best to use water that has been freshly boiled. Through a small metal pipe carbonic acid gas flows into the bath under the cover, but above the surface of the water, and rising through notches in the edge of the opening of the cover fills the funnel and crucible. (See footnote $b$, p. 108.)

One-half to $1 \mathrm{gram}$ of the powder is treated exactly as in $a$ until any carbonates that may be present cease to effervesce. The cover of the crucible is rinsed if need be and the open crucible at once placed in the opening of the bath, the funnel put, in place, the gas current started, as also the flow of water from the reservoir, and the lamp under the bath already full of water is lit. As soon as it can be safely assumed that the air in both funnel and crucible has been displaced a measured volume of strong hydrofluoric acid (5 to 7 $\mathrm{cm}^{3}$ ) is poured into the crucible through a funnel of platinum or rubber and a platinum stirring rod is inserted through the stem of the glass funnel into the crucible. In absence of a suitable funnel for the acid, the glass may be momentarily raised for the introduction of the acid, but not before the crucible has become filled with carbon dioxide.

When steam issues strongly from the funnel, the gas current from the generator is stopped and the apparatus left to itself for an hour, except for occasional stirring of the powder. When no gritty matter 
can longer be felt or when experience tells that decomposition must be complete, the gas current is again turned on, the lamp extinguished, and the water flow increased to the capacity of the outlet tube from the bath. In fifteen minutes the crucible and contents should be cool. The rod is then removed and placed in the titration vessel containing freshly boiled water (usually about $300 \mathrm{~cm}^{3}$ ) and addi-

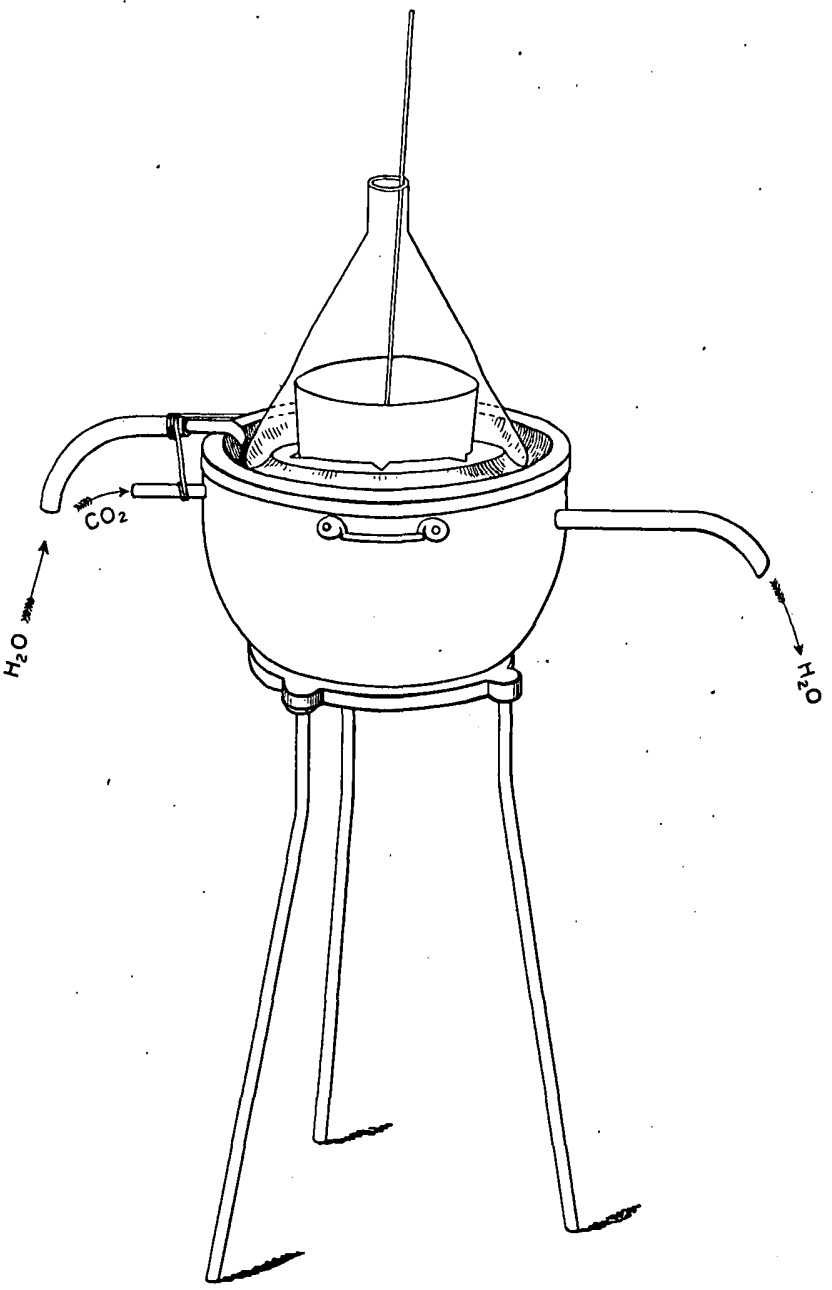

Fra. 21.-Cooke's apparatus for ferrous-iron determination.

tional sulphuric acid, which is already under the burette, the contents of the crucible quickly follow with the rinsings, and the titration is made as in $\alpha$. Until the operator has become experienced a duplicate determination should always be made. It is to be noted that the crucible is always much fuller at the end of the heating than when first placed on the bath, because of condensation of steam. 
Instead of allowing the crucible to cool in a current of carbon dioxide, it may be at once transferred to the titration dish and permanganate run in as above directed. If carefully done, the experiments already referred to seem to show that the results by this variation of the method may be a trifle better than those obtained after cooling.

Those inexperienced with the method in either variation should not fail to test it first with solution of ferrous sulphate that has been standardized without hydrofluoric acid, and in practice duplicate determinations should be made if possible.

r. According to Treadwell.-The specially ground powder in a dish or capacious crucible is placed, after stirring up with 5 or $10 \mathrm{~cm} \cdot{ }^{3}$ of dilute sulphuric acid, on a support of glass or platinum $a$ in the lead box $\mathrm{C}$, which in turn is supported in a paraffin bath B, fig. 22. After covering the box with its leaden cover there is introduced through A a rapid current of carbon dioxide ${ }^{a}$ until the air is displaced.

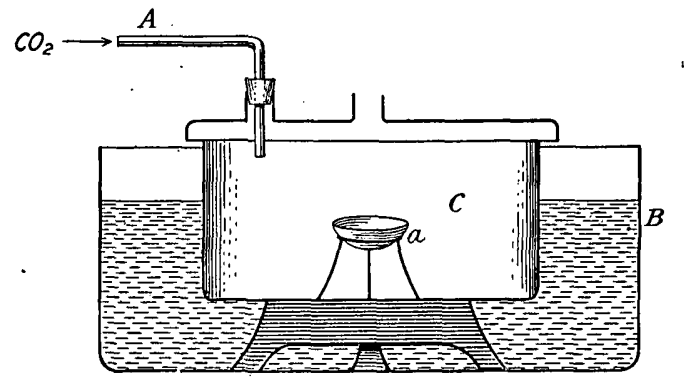

FiG. 22.-Treadwell's apparatus for ferrous-iron determination. B, Paraffin bath; C, lead box with closely fitting cover of same material; $a$, platinum dish resting on glass support.

The cover is then quickly raised. Concentrated hydrofluoric acid ( 5 to $7 \mathrm{~cm}^{3}$ ) is added to the dish and the cover is replaced without interrupting the current. The contents of the dish are stirred with a platinum rod through the opening directly over the dish. The bath has meanwhile been brought to about $100^{\circ}$, a temperature which is maintained with occasional stirring for from one-half to one hour or more, according to the resisting quality of the powder. Then the temperature is raised to about $120^{\circ}$ for an hour to expel most of the hydrofluoric acid. The flame is then extinguished and the solution allowed to cool as quickly as may be, still in the gas current. When cool, the contents of the dish are titrated, as in $a$.

Although the chief object of this method is to remove the excess of hydrofluoric acid and thus avoid one source of error in titration, it may be said, so far as my own limited experience with it goes, that the expulsion is by no means perfect at $120^{\circ}$ and that the long duration of the experiment renders it likely that lower results will be 
obtained than with the variations of the hydrofluoric-acid method described in $\alpha$ and $\beta$, by reason of greater oxidation of ferrous iron.

The need of a fine powder is a serious objection, as with the Cooke method.

\section{E. UNCERTAINTIES OF THE FERROUS-IRON DETERMINATION.}

From the foregoing it is apparent that despite the utmost care in practical manipulation, the exact determination of ferrous iron in rocks is one fraught with extraordinary difficulties and uncertainties. Only in absence of decomposable sulphides and carbonaceous matter and when the amount and condition of vanadium are known and relatively coarse powders can be used, is it permissible to regard the result as fairly above suspicion.

\section{ALKALIES}

\section{A. THE J. LAWRENCE. SMITH METHOD.}

a. ITS ADVANTAGES.

The various methods of getting at the alkalies in insoluble silicates differ more in the mode of attack of the mineral powder and in the immediately subsequent. treatment than in the final stages. With very few exceptions, since the early days of the Survey's existence all alkali determinations have been made by the method of J. Lawrence Smith, ${ }^{a}$ which is far more convenient than and fully as accurate as those in which decomposition is effected by hydrofluoric and sulphuric acids, or by bismuth, lead, or boric oxides. One of its chief advantages is the entire elimination of magnesia at the start.

\section{b. REAGENTS AND APPARATUS.}

Decomposition of the powder is effected by heating it with its own weight of ammonium chloride and eight times as much precipitated calcium carbonate.

The ammonium chloride used must be purified, preferably by sublimation, or made by neutralizing pure ammonia by pure hydrochloric acid, and the calcium carbonate is best obtained from pure calcite by solution and reprecipitation. However obtained, this last is rarely free from alkalies, which must be estimated once for all in a blank test in order to apply a correction. Eight grams of the carbonate will yield usually from 0.0012 to 0.0016 gram of alkali chlorides, almost entirely the sodium salt, but the amount has been brought down to half the above by very long washing. This correction may be admitted at once to be a defect of the method, but it is one easily applied with safety. It is not at all certain that all the alkali found

a Am. Jour. Sci., 2d ser., vol. 50, 1871, p. 269; Am. Chemist, vol. 1, 1871; Annalen Chem. und Pharm., vol. 159,1871 , p. 82 . 
in the blank test comes from the carbonate. Much hot water is used for the analysis and a hot-water bottle, even of Jena glass, will afford weighable amounts of alkali.

The ignition may be made in a covered crucible of ordinary shape and of about 20 to $30 \mathrm{~cm}^{3}$ capacity, heated to full redness for not more than two-fifths of its height, but the heat has to be kept so low in this case to avoid loss by volatilization that perfect decomposition is not always assured. ${ }^{a}$ Hence, to avoid waste of time in very fine grinding, the form of crucible with cap originally advocated by Smith

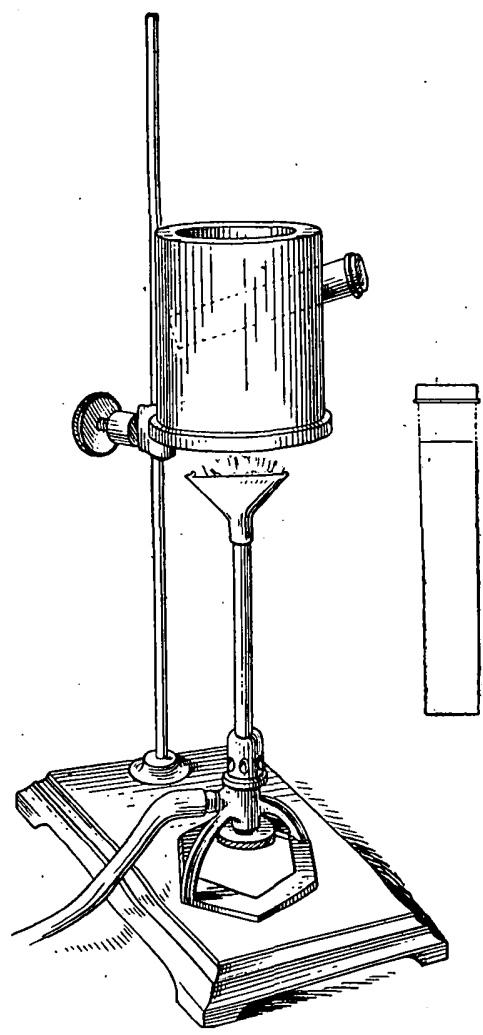

Fig. 23.-The J. Lawrence Smith crucible for alkali determinations. For dimensions see text. is very much to be preferred, since it permits, when set at an angle through an opening in the side of a fire-clay cylinder, the application of the full heat of two burners, and perfect decomposition invariably results without the need of extraordinary care in grinding. The crucible used in this laboratory (fig. 23) for one-half gram of rock powder and 4 grams of calcium carbonate is $8 \mathrm{~cm}$. long, $1.8 \mathrm{~cm}$. wide at the mouth, and $1.5 \mathrm{~cm}$. at the bottom. For double the amounts or more the dimensions are $8 \mathrm{~cm} ., 2.5 \mathrm{~cm}$., and $2.2 \mathrm{~cm}$. The weights are 25 and 40 grams.

\section{c. TREATMENT OF THE MINERAL POWDER.}

Perfectly satisfactory results are to be obtained with but half a gram of rock powder. This is weighed out, ground fine in a large agate mortar, mixed with its own weight of sublimed ammonium chloride, and the two thoroughly ground together. Then nearly all of 4 grams of calcium carbonate is added and the grinding continued till a thorough mixing has resulted. The contents of the mortar are transferred to the long crucible, the rest of the carbonate being used for rinsing off mortar and pestle. The crucible is then capped and placed in an inclined position in a clay cylinder (fig. 23) or through a hole in a piece of stout asbestos board clamped in a vertical position, and heated for about ten minutes by alow flame placed at considerable distance beneath. As soon as the odor of ammonia is no

\footnotetext{
a R. L. Steinlen (Chem. Zeitung, vol. 29, 1905, p. 364) describes a simple cooler for the lid of an ordinary crucible to obviate this difficulty. E. W. Morley (private communication) uses with good effect as a lid a closely fitting small platinum dish filled with cold water.
} 
longer perceptible the nearly full flame of two Bunsen burners is substituted, and continued for forty to fifty minutes. The sintered cake ${ }^{a}$ detaches readily from the crucible as a rule; if not, it is softened up in a few minutes by hot water and digested in a dish until thoroughly disintegrated. It is first washed by decantation, and any lumps are broken up by a pestle, then thrown on the filter and well washed with hot water. The residue should dissolve completely in hydrochloric acid without showing the least trace of unattacked mineral, not even of quartz, though sometimes a few black particles of iron ores will dissolve but slowly.

\section{d. SEPARATION OF CALCIUM AND SULPHURIC ACID.}

All but a trifling amount of the calcium is separated at boiling heat in a large platinum dish by double precipitation by ammonia and ammonium carbonate. The combined filtrates are evaporated to dryness and the ammonium salts are carefully driven off (B, p. 32). From the aqueous solution of the residue-but a few cubic centimeters in bulk-the rest of the calcium is thrown out by ammonia and ammonium oxalate, the last being more effective than the carbonate. The filtrate, caught in an untared platinum crucible or small dish, is evaporated to dryness and gently ignited; the residue is moistened with hydrochloric acid to decompose any alkali carbonate that may have been formed, again evaporated, ignited, and weighed. On solution in water a few tenths of a milligram of fixed residue is invariably left, which should be collected, ignited, and weighed in the same crucible or dish in order to arrive at the weight of the chlorides.

If the rock contains sulphur this will be in part found with the chlorides as sulphate. Therefore, if the sulphur is at all considerable in amount it must be removed by a drop of barium chloride before the final precipitation of the calcium. The excess of barium is removed by ammonium carbonate and the last of the calcium by ammonium oxalate, as above. If the sulphur is not thus removed there is danger, if not certainty, of the potassium chloroplatinate carrying sodium sulphate. A faint reaction for sulphate can usually be obtained, anyway, if the evaporations have been made on a water bath heated by gas.

\section{e. PRECIPITATION OF POTASSIUM.}

To the solution of the chlorides in a small porcelain ${ }^{b}$ dish an excess of chlorplatinic acid solution is added. The dilution should be such that when heated on the water bath any precipitate that may form

$a$ To avold the formation of a melted cake with silicates high in iron it is advisable to increase the proportion of calcium carbonate. In order to lessen the chance of adhesion to the bottom W. T. Schaller, of the Survey laboratory, finds it well to place a layer of calcium carbonate at the bottom of the crucible before introducing the mixture. Mr. Steiger finds that the slacking of the cake is aided by using but 1 or $2 \mathrm{~cm} .{ }^{3}$ of water at first.

$b$ Preferred to platinum because of the possibility, under certain rare and ill-understood conditions, of the formation of an insoluble platinous compound, probably by reaction between the plctinum of the dish and that of the salt. (See also Bolm, F., Zeitschr. anal. Chemie, vol. 38, 1899, p. 349.) 
wholly redissolves. Evaporation is then carried on till the residue solidifies on cooling. It is then drenched with alcohol ${ }^{a}$ of 80 per cent strength, filtered by decantation through a very small filter and washed by decantation with alcohol of the same strength. The precipitate is not brought onto the filter more than can be avoided. Dish and filter are then dried for a few minutes to remove adhering alcohol, the contents of the former are transferred to a weighed platinum crucible or very small dish, and what still adheres to the porcelain is washed through the filter with hot water into the weighed receptacle. This is now placed on the steam bath and afterwards heated for a short time to $135^{\circ}$ in an air bath. It is very important to cover the dish at first in the air bath, for decrepitation with resultant loss sometimes takes place if this is not done. With rocks low in potash drying at $100^{\circ}$ suffices fully to expel all water. The factor used for reduction of $\mathrm{K}_{2} \mathrm{PtCl}_{6}$ to $2 \mathrm{KCl}$ is 0.307 and of $2 \mathrm{KCl}$ to $\mathrm{K}_{2} \mathrm{O}$, 0.632 . It does not seem necessary with the relatively small amounts of potassium in rocks to make use in place of the first of these of the slightly.different empirical factor given in some text-books, a factor based on the analysis of commercial potassium salts. If thought desirable the potassium salts can be tested spectroscopically for cæsium and rubidium.

\section{f. SODIUM AND LITHIUM.}

If it is desired to check the sodium, ordinarily determined by difference, this can readily be done by evaporation of the alcoholic filtrate from the potassium chloroplatinate, reduction of the platinum by hydrogen or otherwise as mentioned in the footnote, ${ }^{b}$ filtration, evaporation, and weighing of the sodium chloride. A check made in this way should agree with the determination by difference within half a milligram on the weight of the chloride. Lithium may then be tested for by the spectroscope, or in case no direct weighing of the sodium chloride is made, the evaporated alcoholic solution may. be examined directly. Lithium is almost invariably present, but almost never in amount to warrant quantitative estimation.

\footnotetext{
$a$ The claim of H. Precht (Zeitschr. anal. Chemie, vol. 18, 1879, p. 513) that absolute alcohol is preferable, especially if evaporation has been carried to dehydration of the sodium salt, has been disputed by $A$. Atterberg and seems to have been conclusively disproved by J. Morozewicz (Anzeig. Akad. Wiss. Krakau, 1906, p. 796). According to Morozewicz, absolute alcohol partly decomposes $\mathrm{Na}_{2} \mathrm{PtCl}_{6}$ with formation of $\mathrm{NaCl}$, which goes to increase the weight of the $\mathrm{K}_{2} \mathrm{PtCl}_{6}$. A further advantage of the weaker alcohol lies in the fact that no more chlorplatinic acid than the amount theoretically required to convert both sodium and potassium to chlorplatinates need be used. In cases calling for the highest degree of accuracy Precht's solubility coefficient for $\mathrm{K}_{2} \mathrm{PtCl}_{6}$ in 80 per cent alcohol can be used, namely, $1: 26400$.

$b$ When haste is not an object, this way of Bunsen's for removing platinum from the chlorides of the alkalies is by far the neatest and most satisfactory. The small flask containing the solution is placed in a water bath and attached to a hydrogen generator. After expelling all air the flask is closed, without breaking connection with the generator, and left to itself, except for occasional light shaking up, till reduction is accomplished. A more expeditious and very satisfactory reduction is effected by evaporating the solution to dryness with metallic mercury, then heating to expulsion of the excess of mercury and of its chloride (Sonstadt, E., Jour. Chem. Soc., vol. 67,1895 , p. 984, thus reduces potassium chlorplatinate in order to weigh its platinum).
} 
Should it be so, however, the very excellent Gooch method (summarized below) of separation by amyl alcohol is to be followed. In rock analysis there need be no fear of enough lithium falling with the potassium to cause any concern.

If ammonium carbonate alone has been relied on to separate all calcium (see d, p. 173) the few tenths of a milligram of calcium chloride that escaped precipitation should now be separated from the sodium and the proper correction made.

$\alpha$. Gooch's method ${ }^{a}$ for separating lithium.-This method is described as follows:

To the concentrated solution of the chlorides amyl alcohol is added and heat is applied, gently at first, to avoid danger of bumping, until the water disappearing from solution and the point of ebullition rising and becoming constant for some minutes at a temperature which is approximately that at which the alcohol boils by itself, the chlorides of sodium and potassium are deposited and lithium chloride is dehydrated and taken into solution. At this stage in the operation the liquid is cooled and a drop or two of strong hydrochloric acid added to reconvert traces of lithium hydrate in the deposit, and the boiling continued until the alcohol is again free from water. If the amount of lithium chloride present is small, it will now be found in solution and the chlorides of sodium and potassium will be in the residue, excepting the traces, for which correction will be made subsequently. If, however, the weight of lithium chloride present exceeds 10 or $20 \mathrm{mg}$., it is advisable at this point, though not absolutely essential to the attainment of fairly correct results, to decant the liquid from the residue, wash the latter a little with anhydrous amyl alcohol, dissolve in a few drops of water; and repeat the separation by boiling again in amyl alcohol. For washing, amyl alcohol, previously dehydrated by boiling, is to be used, and the filtrates are to be measured apart from the washings. In filtering it is best to make use of the perforated crucible and asbestos felt, and apply gentle pressure. The crucible and residue are ready for the balance after drying for a few minutes directly over a flame turned low. The weight of insoluble chlorides actually obtained in this manner is to be corrected by the addition of 0.0004 .1 gram for every $10 \mathrm{~cm} .{ }^{3}$ of amyl alcohol in the filtrate, exclusive of washings, if the insoluble salt is entirely sodium chloride, 0.00051 gram for every $10 \mathrm{~cm} \cdot{ }^{3}$ if potassium chloride constitutes the residue, and, if both sodium and potassium chlorides are present, 0.00092 gram; but $* * *$ the entire correction may in any case be kept within very nar row limits if due care be given to the reduction of the volume of residual alcohol before filtration. The filtrate and washings are evaporated to dryness, treated with sulphuric acid, the excess of the latter driven off, and the residue ignited to fusion and weighed. From the weight thus found the subtraction of 0.0005 gram is to be made if sodium chloride constitutes the precipitate, $0.00059 \mathrm{gram}$ if potassium chloride alone is present in the residue, and $0.00109 \mathrm{gram}$ if both these chlorides are present, for every $10 \mathrm{~cm} .^{3}$ of filtrate, exclusive of washings.

Amyl alcohol is not costly, the manipulations of the process are easy, and the only objectionable feature-the development of the fumes of amyl alcohol-is one which is insignificant when good ventilation is available.

The process has been used for some months frequently and successfully, by others as well as by myself, for the estimation of lithium in waters and minerals.

a Proc. Am. Acad. Arts Sci., 1886, p. 177; Bull. U. S. Geol. Survey No. 42, 1887, pp. 85-86; Chem. News vol. 55,1887 , pp. $18,29,40,56,78$; Am. Chem. Jour., vol. 9,1887 , p. 33 . 


\section{B. PRELIMINARY TREATMENT BY OTHER METHODS.}

When, as may happen in rare instances, it is necessary to estimate alkalies in the main portion after elimination of silica, alumina, lime, etc., in one of the usual ways, the question of a suitable method for separating magnesium becomes important.

\section{a. THE MERCURIC OXIDE METHOD.}

The old barium hydroxide method is not to be recommended. The mercuric oxide method of Zimmermann, whereby the magnesia is precipitated from solution of the chlorides by moist, freshly precipitated, and alkali-free mercuric oxide, can give satisfactory results. The oxide is added in excess to the solution in a platinum crucible and evaporated to dryness. Then the mercuric chloride and most or all of the excess of oxide are expelled by cautious heating. On leaching with water the magnesia remains on the filter. With more than 1 per cent of magnesia the operation must be repeated (Dittrich).

\section{b. THE AMMONIUM CARBONATE METHOD.}

Lately the once favored method of precipitating the magnesium by neutral ammonium carbonate in concentrated solution has been again recommended. ${ }^{a}$ The magnesium solution must be as strongly concentrated as possible, and a great excess of ammonium carbonate solution must be used. A voluminous precipitate forms, which dissolves on vigorous stirring if enough of the precipitant was used. After a time a crystalline precipitate falls-a double carbonate of magnesium and ammonium-which is insoluble in the concentrated solution of ammonium carbonate. Allow to stand for six to twentyfour hours. Wash with the concentrated ammonium carbonate solution. It is no exercise of undue caution to redissolve and reprecipitate, to make sure of getting all alkali in the filtrate, especially potassium.

F. A. Gooch and E. A. Eddy ${ }^{b}$ find that, when carried out as directed above by Wülfing, precipitation of the magnesium is incomplete, but that this defect can be remedied by addition of alcohol. Of several procedures devised they prefer the following as most convenient for the proportions of salts they used, namely, about 0.15 gram $\mathrm{MgO}$ and 0.1 to 0.2 gram alkali chlorides.

A solution is first prepared by saturating with ammonium carbonate a mixture of $180 \mathrm{~cm} \cdot{ }^{3}$ ammonia [density 0.92 ?], $800 \mathrm{~cm} \cdot{ }^{3}$ water and $900 \mathrm{~cm}$. absolute alcohol. The solution containing the chlorides

$a$ Wülfing, E. A., Ber. Deutsch. chem. Gesell., vol. 32, 1899, p. 2214. The neutral carbonate is prepared by dissolving 230 grams of ammonium carbonate in $180 \mathrm{~cm} \cdot{ }^{3}$ of ammonia of 0.92 specific gravity and enough water to make 1 liter. This is sometimes called Schaffgotsch's solution.

b Am. Jour. Sci., 4th ser., vol. 25, 1908, p. 444; Chem. News, vol. 97, 1908, p. 280. 
of magnesium and the alkalies is brought to a volume of about $50 \mathrm{~cm} .{ }^{3}$ and an equal amount of absolute alcohol is added. Precipitation is made by addition of $50 \mathrm{~cm} .^{3}$ of the saturated ammoniacal ammonium carbonate solution containing 50 per cent of alcohol, and the mixture is allowed to stand twenty minutes, with stirring for five minutes. If the amount of alkali salts originally present is small, the precipitate may be collected on asbestos in a perforated crucible, washed with the precipitant, dried, ignited, and weighed as magnesium oxide. When the amount of alkali salt originally present is large, the precipitate may be freed from traces of the alkali salt by pouring off the supernatant liquid through the prepared asbestos filter, dissolving the precipitate, and precipitating ammonium-magnesium carbonate as at first. The second precipitate, collected upon the filter originally used, leaves, upon ignition, practically pure magnesium oxide.

\section{THE AMYL ALCOHOL METHOD.}

Under certain circumstances, notably absence of lithium, the method of Gooch developed by Riggs ${ }^{a}$ may be satisfactory. It is similar to that of Gooch for separating lithium from sodium and potassium chlorides by amyl alcohol, and involves the same solubility corrections for the alkali chlorides above noted (f. $\alpha$, p. 175) in the description of Gooch's method.

\section{DIRECT SEPARATION OF POTASSIUM BY SODIUM COBALTINITRITE.}

It may sometimes happen that of the alkalies present the percentage of potassium alone is desired. In this case use may be made of the method of .W. Autenrieth, ${ }^{b}$ which has afforded its author results that are in good agreement with those afforded by the J. Lawrence Smith method.

\section{a. PREPaRATION OF THE REAGENT.}

Crystallized cobalt nitrate (30 grams) is dissolved in $60 \mathrm{~cm} .{ }^{3}$ of water. To the solution is added $100 \mathrm{~cm}^{3}$ of a saturated solution of sodium nitrite (corresponding to 50 grams of $\mathrm{NaNO}_{2}$ ) and $10 \mathrm{~cm}^{3}$ of glacial acetic acid. Effervescence, with escape of nitric oxide, ensues and the color changes to dark yellow-brown. Some potassium cobaltinitrite usually separates, which, after one to two days, is filtered oft. The reagent undergoes hardly any decomposition in three to four weeks if kept in a dark bottle. It is still available if a few drops, when added to $5 \mathrm{~cm} .{ }^{3}$ of water containing one drop of 10 per cent $\mathrm{KCl}$ solution, cause almost immediately a yellow precipitation. 
b. APPLICATION OF THE METHOD.

The mineral powder ( 1 to 3 grams) is decomposed with hydrofluoric and sulphuric acids, the excess of the latter acid is volatilized, the dry residue is ground and digested with 30 to $40 \mathrm{~cm}^{3}$ of water. To the solution, contained in a capacious beaker of Jena glass (or in a platinum dish), is added $10 \mathrm{~cm} .^{3}$ of a saturated solution of potassiumfree sodium acetate ${ }^{a}$ and the mixture is boiled for from ten to fifteen minutes. The precipitate of basic acetates is filtered and washed a few times with hot water. The filtrate is evaporated to. a bulk of 15 to $20 \mathrm{~cm}^{3}$, then, according to the amount of potassium present; 5 to $10 \mathrm{~cm}^{3}$ of the cobalt reagent are added and, after stirring, the liquid is let stand for twenty-four hours, cold. The yellow precipitate is filtered, washed with cold water containing a little of the reagent, and dried at $100^{\circ}$. Since it is not free from sodium, it is useless to weigh it in this condition.

The further treatment differs according as the potassium is to be weighed as chlorplatinate or as perchlorate.

$\alpha$. Determination as chlorplatinate.-The precipitate is removed from the paper, which is ashed in a platinum crucible; the precipitate is then added and heated to dull redness for a few minutes. The resulting alkali nitrites are extracted with hot water, filtered from the cobalt oxide, evaporated to dryness with strong hydrochloric acid, and the potassium is determined as in A. e, p. 173 .

$\beta$. Determination as perchlorate.-The yellow precipitate is transferred as far as possible to a small porcelain dish, the paper is ashed and its aqueous extract is added to the contents of the dish. To this is then added, drop by drop, a few cubic centimeters of hydrochloric acid (sp. gr. 1.124), the blue solution is evaporated to complete dryness and treated with 5 to $6 \mathrm{~cm}^{3}$ of perchloric acid (sp. gr. 1.124) free from sulphuric acid. The solution is evaporated to dryness, treated again with $6 \mathrm{~cm} .^{3}$ of perchloric acid, again evaporated to dryness, and finally heated over a free flame till copious vapors of perchloric acid come off. The residue of perchlorates is then extracted with $10 \mathrm{~cm}^{3}$ of 96 per cent alcohol, containirg 0.2 per cent of perchloric acid, and allowed to stand for some minutes. The insoluble, white, sandy potassium salt is collected in a Gooch crucible having a thick felt and washed, first with a few cubic centimeters of the alcoholic solution, then with a mixture (usually $20 \mathrm{~cm}^{3}$ suffice) of equal parts alcohol and ether, till a few drops of the filtrate show on evaporation no residue. The precipitate is dried at $120^{\circ}$ to $130^{\circ}$ to constant weight.

$a$ Five grams dissolved in water should give no yellow precipitate with the cobalt reagent after twentyfour hours. 


\section{CARBON DIOXIDE, CARBON.}

\section{A. QUALITATIVE TEST FOR CARBON DIOXIDE.}

In the preliminary qualitative test for carbon dioxide, it must be remembered that while calcite gives off its carbon dioxide on treatment with cold acid, dolomite and siderite do not, and hence warming should not be omitted; otherwise, a few tenths per cent of carbon dioxide can very well be overlooked. Moreover, the powder should first be agitated with a little hot water, to remove all entangled air which might otherwise be mistaken for carbon dioxide. In order not to overlook traces the test is best made in a test tube, first boiling the powder with a little water, then cooling and adding dilute hydrochloric acid. If effervescence is immediate the presence of calcite is assured; if escape of bubbles takes place only on warming the carbonate is not calcite. In order to make sure of the reaction it may be necessary to use a pocket lens, holding the test tube in an inclined position and looking down in order better to see the minute bubbles as they stream up along the upper glass wall. It is, of course, important not to mistake escaping hydrogen sulphide for carbon dioxide.

\section{B. QUANTITATIVE TESTS FOR CARBON DIOXIDE.}

a. DIRECT METHOD.

For the quantitative determination of carbon dioxide an apparatus permanently set up is used, of which several forms have been described by different writers. ${ }^{a}$ The one depicted in fig. 24 is a compact arrangement at present used in the Survey laboratory.

The rock powder ( 1 to 5 grams) is boiled with dilute hydrochloric acid in a small Erlenmeyer flask attached to an upward-inclined condenser, whence, after passing through a drying system-calcium chloride, anhydrous copper sulphate to retain hydrogen sulphide from decomposable sulphides and any hydrochloric acid that may pass over, then calcium chloride again - the carbon dioxide is caught by absorption tubes filled with soda lime followed by calcium chloride. Of course arrangement is made for a current of $\mathrm{CO}_{2}$-free air with which to sweep out the apparatus before and after the experiment, and for a slow current during its continuance. The results are very accurate and the determination can be quickly carried out.

The manipulations are as follows: Hot water is poured upon the powder in the flask; this is attached to the condenser, and a current of $\mathrm{CO}_{2}$-free air is forced through the whole system, except the weighed absorption tubes, until the original air has been displaced, the observation bulbs $\mathrm{E}$ being attached directly to the drying system $\mathrm{B}$. The

a For a simple and convenient form, see Treadwell's Quantitative analysis, American edition, p. 298. 
stopcock in the separatory funnel is then closed, the latter half filled with hydrochloric acid (1:1), the rubber stopper of the funnel replaced, the absorption tubes inserted between $\mathrm{B}$ and $\mathrm{E}$, and the acid allowed to flow into the flask, slowly if there is much carbon dioxide, rapidly if there is but little. When effervescence diminishes in the former case, at once in the latter, the lamp is lighted and the flow of water

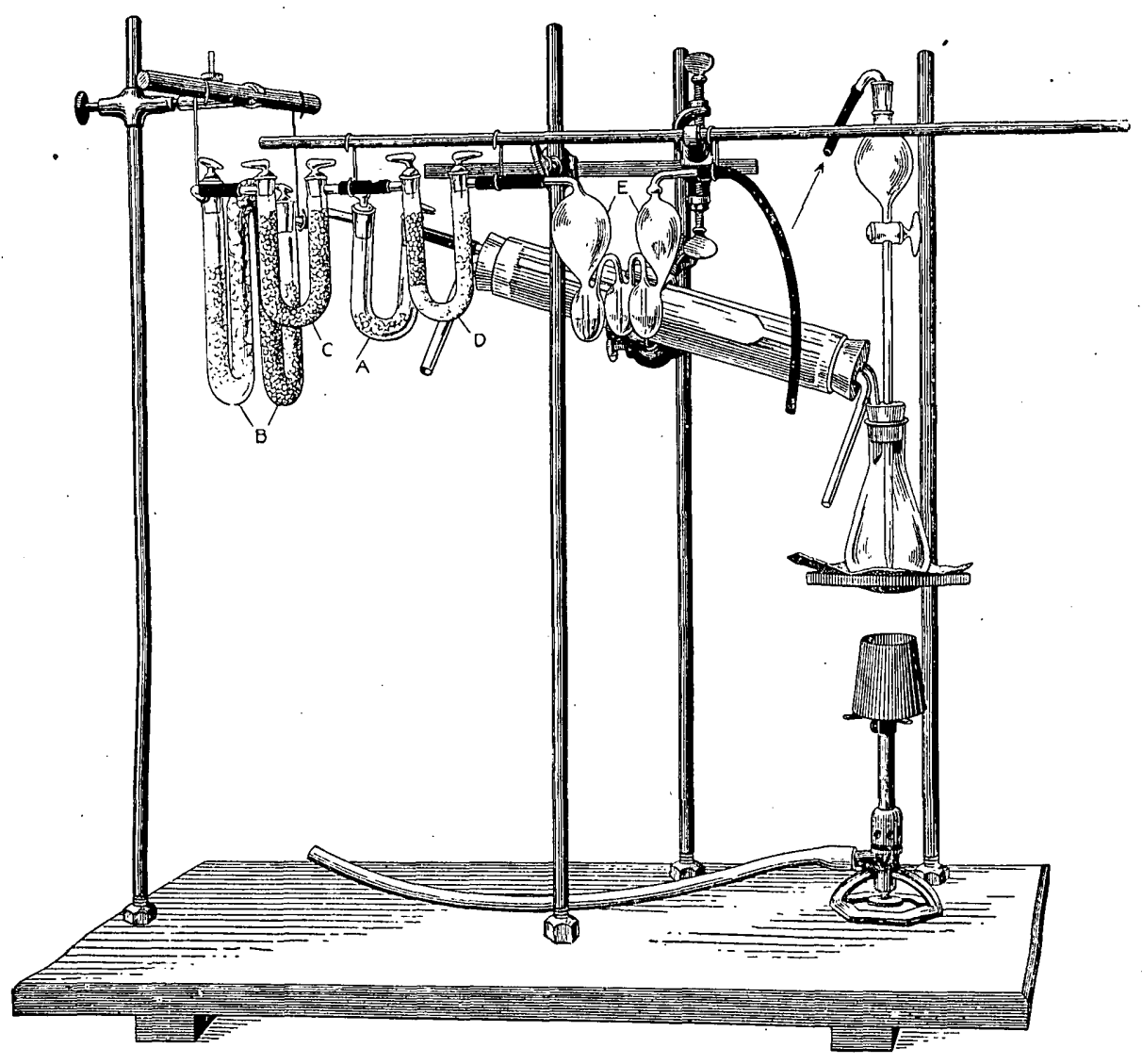

Fig. 24.-Compact form of apparatus for determination of carbon dioxide. A, Protective calcium chloride tube, frequently refilled, to retain most of the moisture from the condenser. B, Double U-tube, filled in the center with pumice impregnated with anhydrous copper sulphate and at both ends with calcium chloride. $\mathrm{C}$ and $\mathrm{D}, \mathrm{A}$ bsorption tubes, $\mathrm{C}$ and one-third of $\mathrm{D}$ containing soda lime, followed in $\mathrm{D}$ by calcium chloride. C needs to be refilled before the soda lime in $\mathrm{D}$ has absorbed much if any carbon dioxide, $\mathrm{D}$ then requires only occasional refilling, but it will not do to use it indefinitely, since the calcium chloride in it becomes less and less effective as it takes up the water set free from the soda lime in C during its conversion to carbonate. E, Observation bulbs containing sulphuric acid to show the rate of gas flow.

through the condenser started. The flame is kept low, so as to secure steady but quiet ebullition, and the gas current is not interrupted, although reduced to a slow rate. With much carbon dioxide the rate of absorption is very readily noted by holding the hand to the soda-lime tubes, which become hot or warm where absorption is 
taking place. A sufficient time having elapsed, the flame is extinguished and the air current increased. When cool the tubes $\mathrm{C}$ and $\mathrm{D}$ are weighed after remaining some time in the balance case.

The soda lime must be porous, not hard and unabsorptive like that sometimes used for the combustion of nitrogenous organic substances.

For the simultaneous determination of carbonates and the carbon of carbonaceous matter see C, page 182 .

It has already been shown under "Water" (5. C. f, p. 82) how in case of need, as paucity of material, the determination of carbon dioxide can be combined with that of water by fusion with lead chromate. Soda-lime absorption tubes then follow the calcium chloride tube in carrying out the determinations there referred to.

\section{b. INDIRECT METHOD.}

The older forms of apparatus for the indirect determination of carbon dioxide are too well known to need description. Some are fragile or cumbersome and great care is requisite to the attainment of good results. The form recently devised by J. L. Kreider ${ }^{a}$ (fig. 25) has the merit of simplicity, cheapness, and ease of manipulation. It is adapted for determining not only carbon dioxide, but also other volatile substances produced by a variety of reactions, as hydrogen resulting from the action of an acid on certain metals, nitrogen from urea, ammonium oxalate or ammonium chloride by the action of sodium hypobromite, etc. Kreider's test results are excellent, though with carbonates generally low to the extent of about 0.06 to 0.1 per cent.

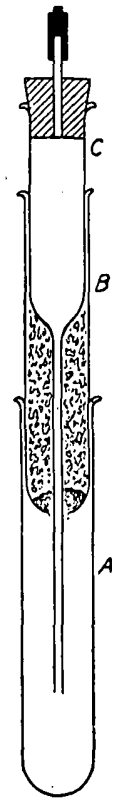

FIG. 25.-Kreider's apparatus for indirect determination of volatile substances.

The apparatus *** is light and easily made from three test tubes. * * * The test tube $A$ is changed in no way from its original form; $B$ is perforated in the bottom with a hole about $1 \mathrm{~cm}$. in diameter and fits tightly within $\mathrm{A}$; and $\mathrm{C}$, so selected that it fits loosely within $B$, is drawn out to a small capillary tube.

When the apparatus is to be used, the capillary of $C$ is pushed through the hole of $B$, packed loosely with cotton; $B$ is filled to the depth of from 6 to $8 \mathrm{~cm}$. (about two-thirds of its contents) with granular calcium chloride, and $\mathrm{B}$ and $\mathrm{C}$ are adjusted as shown.

To the test tube, C, is fitted a one-hole stopper, through which passes a short glass tube which is closed by a rubber cap and plug. Upon removing the plug and applying suction to the short tube the reagent employed to liberate the volatile product to be determined is drawn up through this capillary until $\mathrm{C}$ is sufficiently filled. Upon replacing the plug the reagent remains within $\mathrm{C}$, held by atmospheric pressure.

The tubes $A$ and $B$ may be so selected that very little of the product evolved can escape between them, but in case they fit very loosely a ring of paraffin melted in to the mouth of $A$, about $B$, by means of a hot iron or wire, seals the joint securely. A very convenient way to attach the paraffin is to melt it between $\mathrm{A}$ and another tube, which

a Am. Jour. Sci., 4th ser., vol. 19, 1905, p. 188; Zeitschr. anorg. Chemie, vol. 44, 1905, p. 154. 
fits $A$ as does $B$ and may be removed by a turning motion, leaving the ring into which $B$ will fit, and which then requires very little heating to make a tight joint. If care be used in taking apart A and B at the close of an experiment, such a ring of paraffin remains in place and may be used many times without replacement, being remelted by a touch of the hot wire before every new experiment.

In making the determination the substance under examination is weighed and placed in the bottom of $A$. The reagent to be employed, 10 to $15 \mathrm{~cm}^{3}$, is drawn into $\mathrm{C}$ and held there in the manner described. The test tube $\mathrm{A}$ is slipped over $\mathrm{B}$ and this joint is sealed with paraffin, as has been shown. The apparatus is wiped, placed on the balance, and weighed.

Upon removing the cap from the small tube in $\mathrm{C}$ the reagent runs from $\mathrm{C}$ into $\mathrm{A}$. The volatile product is formed, is forced upward through the drying column of calcium chloride, and escapes through the annular space between B and C. When the action ceases a current of dry air is forced through $\mathrm{C}$ to drive all the volatile products from the apparatus, the cap is then replaced, and the whole placed on the balance to be weighed. The loss in weight represents the volatile product.

\section{CARBon.}

Fusion with lead chromate is commonly resorted to in order to determine the carbon of graphite or carbonaceous matter in rocks and ores, though boiling in presence of sulphuric and chromic acids can be made to yield good results.

If carbonates are at the same time present they can be determined as in B (p. 179), and by a second determination, as just referred to, the total carbon in both forms can be found as $\mathrm{CO}_{2}$, whence that of the graphite is derived by difference. Or, according to G. T. Morgan, ${ }^{a}$ the carbonates are first decomposed as in B by orthophosphoric instead of hydrochloric acid, whereupon chromic acid is added and the carbon oxidized and determined as $\mathrm{CO}_{2}$.

\section{CHLORINE.}

\section{A. CONDITION IN ROCKS.}

Chlorine may exist in rocks in a water-soluble condition, in minerals that are decomposed by nitric acid, and in those not attacked by this acid. In the first form it doubtless exists as infiltrated sodium chloride or as original inclusions in one or more of the constituent minerals; in the second in minerals of the sodalite group and sometimes apatite; in the last chiefly in the scapolites. Where a qualitative test shows the water-soluble form to be present in determinable amount, it must be separately extracted and the amount found deducted from the total as determined in a second portion. Sometimes it may be possible to use the extracted material for the determination of the rest of the chlorine. 


\section{B. DETERMINATION OF WATER-SOLUBLE CHLORINE.}

A suitable amount, which may be several grams, of the powder is extracted with water. The filtrate is very often turbid, a condition which may sometimes be prevented by using double filters or by adding some chlorine-free salt, like sodium nitrate, to the wash water. If the filtrate remains persistently cloudy the precipitation of silver chloride may be proceeded with as usual, after acidifying with nitric acid. It is best to let the precipitate settle over night in order to obtain a clear filtrate on the morrow. It is collected on a small filter, washed with water acidified with nitric acid, dried, and ignited in a very small porcelain crucible without allowing the paper to burst into flame. Most of the chloride will be reduced to metal. On disappearance of all carbon a drop of nitric acid is added and evaporated, then similarly a drop of hydrochloric acid, and the crucible gently heated, but not to fusion of the silver chloride, and weighed. The chloride is then dissolved in a few drops of warm ammonia water and separated by filtration from the siliceous matter, which latter is ignited and weighed in order to get the weight of the chloride.

This method of procedure is permissible even when the original aqueous extract was quite clear, though the operation then stops with the weighing of the chloride. Or; if very small in quantity, the dry paper with its contents is wound up in a tared platinum wire and carefully ignited after Bunsen's manner. The increased weight of the wire is due to the metallic silver of the chloride which has alloyed with it.

\section{DETERMINATION OF ACID-SOLUBLE CHLORINE.}

a. BY NITRIC ACID.

If nitric acid will decompose the chlorine-bearing minerals, or if it is desired to distinguish between the chlorine in soluble and in insoluble minerals, the rock powder may be boiled for a few minutes with dilute chlorine-free nitric acid. The acid should be very dilute in order to cause no loss of chlorine and the boiling should be as brief as possible for the same reason, and also to prevent gelatinization of the silica from soluble silicates. Acid as dilute as 1:40 will readily decompose apatite and probably the minerals of the sodalite group, but it is doubtful if anything like this dilution is called for.

The filtrate does not require evaporation for the removal of dissolved silica, but may be precipitated at once with silver nitrate. The precipitate is treated as in B, or if at all appreciable in amount may be collected on a Gooch crucible. 
b. BY NITRIC AND HYDROFLUORIC ACIDS.

In many cases in order to obtain all the chlorine it is sufficient to attack the powder (which usually must be much finer than the coarse bulk sample) by chlorine-free hydrofluoric and nitric acids in the cold, with occasional stirring, and after filtering through paper fitted into a rubber funnel or large platinum cone to throw down the chlorine by silver nitrate. The presence of nitric acid is necessary, since in its absence ferrous iron in presence of fluorine reduces silver nitrate with deposition of crystallized silver. It may be advisable to redissolve the chloride on the filter in ammonia and to reprecipitate by nitric acid and a drop of silver nitrate. It seems difficult to obtain in the market hydrofluoric acid free from a trace of hydrochloric.

\section{DETERMINATION OF CHLORINE BY ALKALI FUSION.}

Chiefly for the last reason, in order to make sure of getting all the chlorine, it is best to fuse with chlorine-free sodium potassium carbonate, or even sodium carbonate alone, first over the full burner, then for a moment or two over the blast, to leach with water, acidify in the cold with nitric acid, and precipitate by silver nitrate without preliminary separation of silica. After acidification in the cold, except in a very concentrated solution, no precipitation of silica need be feared. The treatment of the precipitate will be as in B and C, according to its amount and freedom from contamination.

\section{FLUORINE (SILICA IN PRESENCE OF FLUORINE).}

\section{A. IMPERFECTION OF THE QUALITATIVE TEST FOR FLUORINE.}

There is no direct qualitative test which will reveal with certainty the presence of fluorine in rocks. Heating the powder before the blowpipe with sodium metaphosphate on a piece of curved platinum foil inserted into one end of a glass tube, or in a bulb tube, is not to be relied on in all cases. While as little as 0.1 per cent of fluorine can sometimes be thus detected with ease, much larger amounts in another class of rocks may fail to show. (See C for indirect method.)

\section{B. DIRECT QUANTITATIVE DETERMINATION OF FLUORINE.}

a. THE METHOD OF BERZELIUS.

For the reason that the fluorine minerals occurring in rocks are mostly attacked but partly, if at all, by strong hot sulphuric acid, none of the methods depending on the volatilization of silicon fluoride can be used. There is further reason that even with decomposable fluorides it is impossible to obtain quantitative results in presence of amorphous silica or silicates. ${ }^{a}$ Only with quartz powder is volatili- 
zation perfect. The method to follow is that of Berzelius, which, though not difficult, requires great attention to details and even then affords low results. (See c for causes of error.) It admits of the determination of silica at the same time, and is in fact the only method available for silica in fluorides.

Two grams of the rock powder are fused with four or five parts of sodium potassium carbonate free from fluorine, avoiding the use of a blast if possible. For minerals rich in fluorine and low in silica it may be necessary to add pure silica before fusing in order to effect complete decomposition of the fluoride, just as with the alkaline-earth phosphates. But this will probably never be required in ordinary rock analysis. To the aqueous extract, containing the fluorine and usually much of the silica besides other bodies, several grams of ammonium carbonate are added, the liquid is digested at a gentle heat $\left(40^{\circ}\right)$ - for some time, and on cooling more carbonate is introduced. ${ }^{a}$ After twelve hours the precipitate is collected and washed with water containing ammonium carbonate. From the filtrate the excess of the latter is expelled by evaporating nearly to dryness and the somewhat diluted solution is brought toward neutrality as follows, according to the directions of Treadwell. A few drops of phenolphthalein are added, then nitric acid (not hydrochloric in this case, see below) till the red color disappears. The solution is now boiled, the color reappearing, to be again discharged by acid on cooling. These operations are repeated till it requires but 1 to $1 \frac{1}{2} \mathrm{~cm} .{ }^{3}$ of $2 \mathrm{~N}$ acid to discharge the color. There is now added 1 to $2 \mathrm{~cm}^{3}$ of an ammoniacal solution of zinc oxide, ${ }^{b}$ and the liquid is boiled till the ammonia is wholly expelled. The precipitate, containing the last of the silica and some phosphorus, in addition to that thrown down with alumina by ammonium carbonate, is separated and washed with water.

The above-prescribed use of nitric instead of hydrochloric acid for neutralizing is necessitated by the fact that phosphorus, which is almost invariably present, and chromium must still be removed, and this can be done only from a nitric solution, as follows: To the still alkaline solution silver nitrate is added in excess, whereby phosphate,

a The use of ammonium nitrate or chloride, instead of carbonate, for throwing out the silica and alumina is not to be recommended because of loss of fluorine on subsequent evaporation (FI. Rose).

$b$ Made by precipitating pure zinc chloride solution with potassium hydroxide, filtering, washing, and dissolving the precipitate in ammonia. If the rocks are very basic, it may happen that the amount of silica in the alkaline solution of the fusion is so small that ammonium carbonate may be dispensed with and the zinc oxide solution added at once, after neutralizing as above.

Instead of the ammoniacal zinc oxide solution F. Seemann (Zeltschr. anal. Chemie, vol. 44, 1905, p. 343), advocates using the Schaffgotsch solution (p. 176. footnote) in which has been dissolved in the cold 20 grams of freshly precipitated mercuric oxide to the liter. For every $0.2 \mathrm{gram}$ of dissolved silica $100 \mathrm{~cm} .^{3} \mathrm{of}$ this reagent are to be used. The solution is then to be evaporated to complete dryness, the residue taken up with water, filtered and washed, the filtrate again neutralized with hydrochloric acid [nitric in rock analysis-W. F. H.], and again evaporated to remove the last of the silica. Where silica is to be determined at this stage this reagent has the advantage over the zinc solution that direct ignition of the precipitates (under a good draft hood) yields at once silica. Its use is manifestly allowable in rock analysis only after the alumina has first been separated by ammonuim carbonate. 
chromate, chloride if chlorine is present, and carbonate of silver are precipitated. The last serves to correct any acidity resulting from the reaction between the alkaline phosphate and the silver salt, thus producing the neutral solution needed for complete precipitation of the phosphate and chromate. After slight heating and filtering, the excess of silver is removed by sodium chloride and $1 \mathrm{~cm} .{ }^{3}$ of $2 \mathrm{~N}$ sodiumcarbonate solution is added to the filtrate, which is then boiled in a dish of good size with a large excess of calcium-chloride solution. At this stage there must be no ammoniacal salts in solution, otherwise calcium fluoride may be held up. The precipitate consists of a mixture of calcium carbonate and fluoride, the former serving to aid filtration of the latter. The precipitate is collected on paper and washed with hot water, dried, and ignited separately from the filter as far as possible. If the dish used for the precipitation was of platinum it is not necessary to cleanse it from adhering precipitate, but. the contents of the filter are washed back into the dish, the liquid in this is evaporated away, the ash of the filter-added, and the dish heated over a free flame to faint redness for a few moments.

Dilute acetic acid is now poured upon the thus gently ignited mixture in small quantities at a time and in amount slightly in excess of that needed to dissolve the calcium carbonate. When visible action ceases the liquid is evaporated to dryness and the residue taken up with hot water and a drop of acetic acid, filtered, dried, and gently ignited.

The amount of fluoride obtained from rocks being usually very small, a single treatment as above suffices, but for the larger amounts obtained from fluorine minerals a second and even third treatment with acetic acid may be needed, filtering and igniting after each. S. L. Penfield and J. C. Minor ${ }^{a}$ found in such cases that if a great excess of acetic acid is used at the start the results are lower than by the repeated treatment. For considerable amounts, when the loss between two treatments is reduced to not more than half a milligram, F. P. Treadwell and A. A. $\operatorname{Koch}^{b}$ take the next to the last weight as correct.

\section{b. TESTING OF THE CALCIUM FLUORIDE.}

The well-washed and gently ignited calcium fluoride finally obtained in the course of this method should be converted to sulphate as a check on its purity, and at the same time as a qualitative test to ascertain if it really is calcium fluoride by the characteristic odor of the gas given off. Should fluorine be found, and the weight of sulphate not correspond to that of the fluoride, the former should be dissolved in hot nitric acid and tested for phosphorus by ammonium 
molybdate solution. If phosphate is absent the impurity may have been silica or calcium silicate-which of these it would be difficult to decide. In the former case the fluorine might be safely deduced. from that of the sulphate, but not in the latter. If the rock were rich in sulphur it might happen that calcium sulphate would be thrown down with the fluoride, but this should be removed by thorough washing. If not, and it were certainly the only impurity present, the fluorine could be calculated, after conversion of the fluoride into sulphate, by the formula

$\mathrm{CaSO}_{4}-\mathrm{CaF}_{2}: 2 \mathrm{~F}:$ : Diff. between impure $\mathrm{CaSO}_{4}$ and $\mathrm{CaF}_{2}: x$.

It is an exceptional case when there is exact agreement between the weight of fluoride and sulphate, and with the small amounts usually met in rocks the error may be an appreciable one in percentage of fluorine, though of no great significance otherwise.

\section{ACCURACY OF THE METHOD.}

It is evident from the above that if the greatest care is not taken to prevent any kind of contamination of the weighed fluoride the error in the small amounts in question may be very great. If phosphate were present it would not do to apply the check by conversion to sulphate, because the evaporation with sulphuric acid and following ignition would volatilize metaphosphoric acid.

There is another source of error due to the solubility of the fluoride itself in water and acetic acid. F. P. Treadwell and A. A. Koch ${ }^{a}$ have investigated the solvent effect of these and some other reagents. They find that it is possible to detect additional fluorine by repeating the precipitation with calcium chloride in presence of sodium carbonate, and that the total errors are such that for $5 \mathrm{mg}$. $\mathrm{CaF}_{2}$ or less the results are much too low, though the qualitative detection can be still made in $100 \mathrm{~cm} .{ }^{3}$ of solution with only 0.0009 gram $\mathrm{CaF}_{2}$, notwithstanding the fact that the quantitative tests had seemed to indicate that the loss would be about 0.0015 gram $\mathrm{CaF}_{2}$ for every $1.00 \mathrm{~cm} .{ }^{3}$, including wash water. It thus appears that with 1 gram of sample amounts of less than 0.04 to 0.05 per cent of fluorine will entirely escape observation.

A source of error not generally known arises whenever calcium fluoride is ignited in contact with paper. Fluorine escapes to a slight extent and is replaced by oxygen, this reaction being doubtless due to the action of water vapor and not to oxygen of the air.

F. Seeman ${ }^{b}$ has subjected all the known methods of determining fluorine to extended examination, using calcium fluoride as his test material, and declares the Berzelius method to be far inferior to those 
based on volatilization of the fluorine as silicon fluoride, by reason of great and varying losses, the causes of which he was unable to satisfactorily trace. He was unable to recover by it more than about 87 to 89 per cent of the fluorine. My tests do not confirm. his very unfavorable results. I was able repeatedly to regain 95 to 98 per cent of the fluorine. To do so requires, however, re-fusion of the residue left after leaching the alkali-carbonate melt, including in the same re-fusion the silica precipitated by ammonium carbonate and ammoniacal zinc oxide, the treatment of the filtrate from the precipitate of calcium carbonate and calcium fluoride with additional sodium carbonate and calcium chloride, and the reevaporation of the acetic acid solutions of the precipitated calcium carbonate.

\section{d. Determination of silica.}

The several precipitates obtained by ammonium carbonate and zinc oxide, together with the residue from the original alkali fusion, are dissolved as far as may be in hydrochloric acid, the papers ignited and their ashes added, and silica separated by the processes detailed in 6 , page 83 . These may be greatly simplified, if bases are not likewise to be determined, by evaporating the first filtrate from the silica with much sulphuric acid as in the last part of f. $\alpha$, page 105.

\section{INDIRECT QUANTITATIVE DETERMINATION OF FLUORINE.}

a. STEIGER'S METHOD.

George Steiger has recently devised in the laboratory of the Geological Survey an indirect method ${ }^{a}$ for the determination of fluorine that seems full of promise.

It is based on the well-known fact that the presence of fluorine has a powerful bleaching effect on the yellow color which is produced by the oxidizing of a titanium solution with hydrogen peroxide. A solution of definite volume is made containing the fluorine to be estimated, also having a known amount of titanium present; this is compared in a colorimeter with a second solution containing an equivalent amount of titanium per cubic centimeter, and the bleaching effect recorded. From the extent of this bleaching, the percentage of fluorine can be calculated.

Although the results obtained are not so accurate as those given by many methods for the estimation of other elements, yet considering the difficulty of the fluorine determination, and the time and labor required by methods now in use, the present one may well be employed, where small quantities of fluorine are to be determined. The operations require not only less skill to carry out, but are fewer in number and take much less time.

Traces of fluorine amounting to several hundredths of 1. per cent are easily detected, and an approximation to the quantity can be made. In amounts up to a few tenths of a per cent this method seems to be more reliable, and if not more than 2 per cent is present, the results compare favorably in accuracy with the standard methods. It is hardly to be expected, however, to find a colorimetric method using only a few milligrams of the material to be determined that will compare in accuracy with the gravimetric methods using much larger quantities, where considerable percentages of fluorine are concerned. 
In rock mixtures containing only a few tenths of a per cent of fluorine, 2 grams of the finely ground rock powder are fused with four or five times its weight of a mixture of sodium and potassium carbonates. It may be necessary in the case of a subsilicic rock to add silica, 50 per cent of which should be present.

From the aqueous leach of the fusion all of the alumina, and most of the silica, are separated by adding ammonium carbonate, heating on the water bath for fifteen or

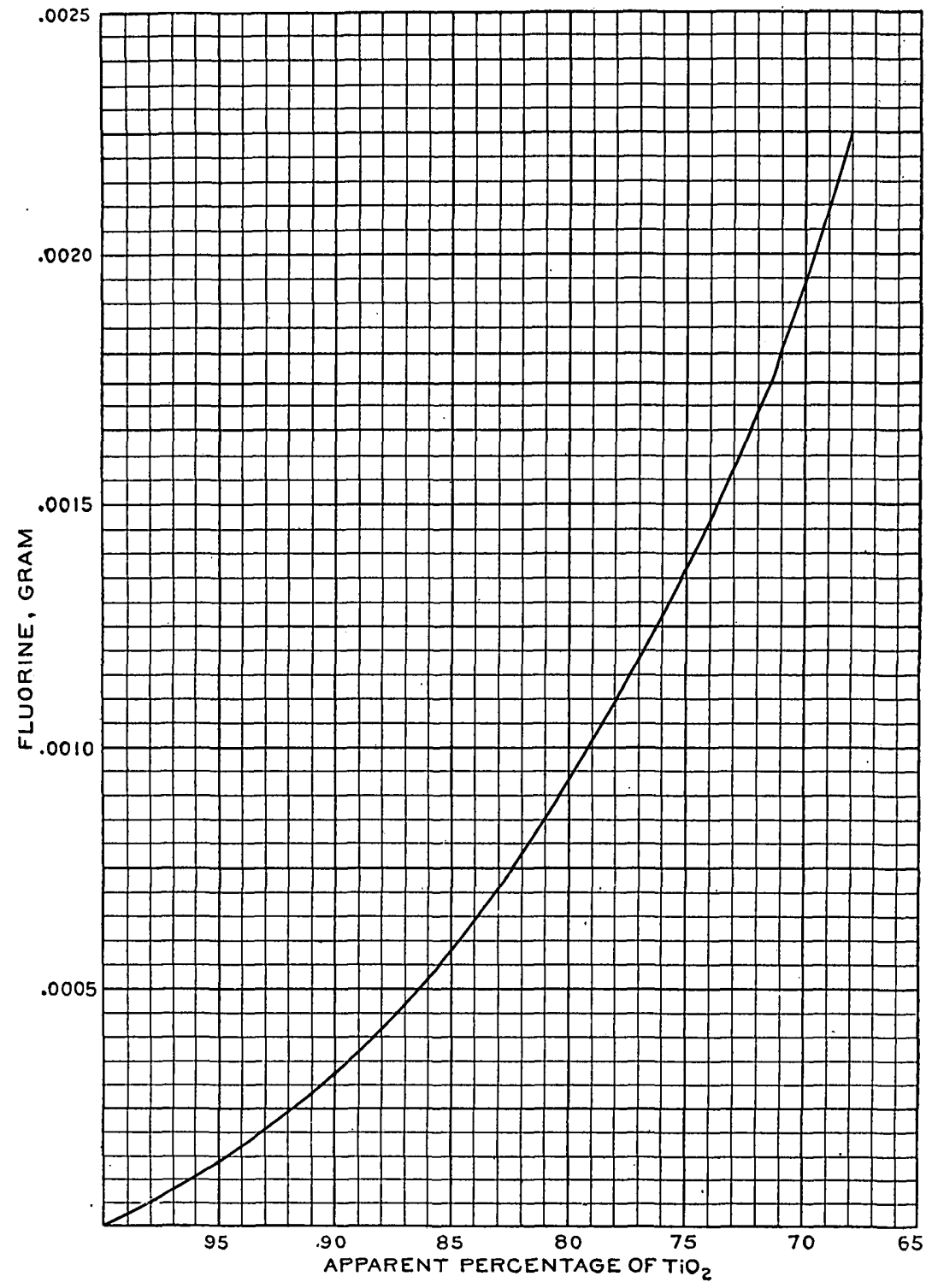

FIG. 26.-Steiger's curve for fluorine.

twenty minutes, allowing to cool an hour or more and filtering. The filtrate is evaporated to small bulk $\left(25\right.$ or $30 \mathrm{~cm}^{3}$ ) and filtered a second time to insure a perfectly clear solution, a condition absolutely necessary for a satisfactory comparison of the color. After this treatment the solution should be entirely free from alumina and contain no more than $25 \mathrm{mg}$. of silica. This amount of silica is not sufficient to interfere with the reaction. 
The solution is now put into a $100 \mathrm{~cm} \cdot{ }^{3}$ measuring flask, sulphuric acid added to almost neutral reaction with care not to add an excess, and well shaken to free from the excess of carbon dioxide, and then fully acidified. Care should be taken not to have an excess of acid present before shaking, for the reason that the escaping gas will carry off some fluorine; even under the above conditions a slight loss occurs. If a considerable amount of fluorine is present, an aliquot part of the solution should be used containing not more than 2 or $3 \mathrm{mg}$. $20 \mathrm{~cm} .{ }^{3}$ of standard titanium sulphate solution $a\left(1 \mathrm{~cm} .^{3}\right.$ of which contains 0.0001 gram $\left.\mathrm{TiO}_{2}\right)$ are now added, together with 2 or $3 \mathrm{~cm} . .^{3}$ of hydrogen peroxide, and the flask filled to the mark with water. The solution is now ready to be compared with the standard. The latter is prepared by using $20 \mathrm{~cm} .^{3}$ of the standard titanium solution, 2 or $3 \mathrm{~cm}^{3}$ of $\mathrm{H}_{2} \mathrm{O}_{2}$ and bringing the volume up to $100 \mathrm{~cm} .^{3}$ with water.

These two solutions containing the same amount of titanium per cubic centimeter should be of the same depth of color, but the one having the fluorine present will be found to be of a lighter shade, owing to the bleaching effect which that element has on a solution of titanium oxidized by $\mathrm{H}_{2} \mathrm{O}_{2}$. The extent of this bleaching is not directly proportional to the amount of fluorine present, but by reference to the curve below, the quantity corresponding to a given bleaching can be found.

The two solutions are now compared in a colorimeter and their ratio recorded. Suppose a ratio, for example, of 100 to 85 -that is, the fluorine present has caused a bleaching of the solution equivalent to 15 per cent of the titanium present; then by finding the point on the abscissa marked 85, with the help of the curve the amount of fluorine $(0.00055)$ can be directly read off on the ordinate.

It is necessary to employ a colorimeter whose error is not more than 2 or 3 per cent.

This method is not suited for large percentages of fluorine.

Mr. Steiger studied the effect on the determination of some of the more important elements that may occur in the test solution. Sodium salts in large amount make the observed reading for the titanium somewhat high, but not enough to affect the results seriously (but see p. 129). Silica in amounts up to 0.1 gram has but little effect, and by the treatment employed its amount is reduced to at most 2 or 3 centigrams. Alumina, which exerts a marked effect, even in small quantities, is readily removed by the preliminary treatment. Phosphoric acid, which bleaches like fluorine, does not interfere unless present in larger amounts than are likely to be encountered in rock analysis.

Various mixtures were made roughly representing commonly cccurring rock mixtures, containing accurately known amounts of fluorine. Results of the fluorine determinations in these mixtures follow:

\begin{tabular}{|c|c|c|c|c|c|c|c|c|c|}
\hline $\mathrm{SiO}_{2}$ & $\mathrm{Al}_{2} \mathrm{O}_{3}$. & $\mathrm{Fe}_{2} \mathrm{O}_{3}$. & $\mathrm{MgCO}_{3}$. & $\mathrm{CaCO}_{3}$ & JaHNF & $\mathrm{O}_{4} \cdot \mathrm{TiO}_{2}$. & F calculated. & $F$ fo & und. \\
\hline 0.60 & 0.20 & 0.05 & 0.03 & 0.05 & $\ldots$ & ..... & 0.0114 & 0.0102 & Gram. \\
\hline " & "، & “" & " & “" & 0.01 & 0.008 & .0052 & .00525 & ، \\
\hline "6 & "، & " & " & 0.10 & " & .01 & .00284 & .0027 & “6 \\
\hline b 0.70 & 0.15 & 0.05 & 0.05 & .05 & " & .005 & .0005 & .0003 & "6 \\
\hline$"$ & "، & ؛ & " & $" 6$ & "، & “ & .00526 & .0056 & "6 \\
\hline "6 & " & " & $" ؛$ & "6 & "6 & " & .00526 & .0040 & " \\
\hline "6 & "6 & " & "6 & " & " & "6 & .00253 & .0020 & " \\
\hline " & "6 & " & "6 & " & " & " & .01228 & .0089 & " \\
\hline "6 & " & “ & "6 & " & ؛ & " & .00516 & .00486 & " \\
\hline 6 & "6 & “" & " & " & " & " & .00536 & .00530 & " \\
\hline
\end{tabular}

$a$ For its preparation, see footnote $b$, p. 130 .

$b$ In this determination the iusion of the mixture was made as usual, and the flucrine added to the leach water before the treatment with ammonium carbonate. 
b. MERWIN'S MODTFICATION OF STEIGER'S METHOD.

$\alpha$. Introductory remairks.-Mention has been made (p. 129) of a paper by H. E. Merwin, ${ }^{a}$ in which it was shown that alkali sulphates and
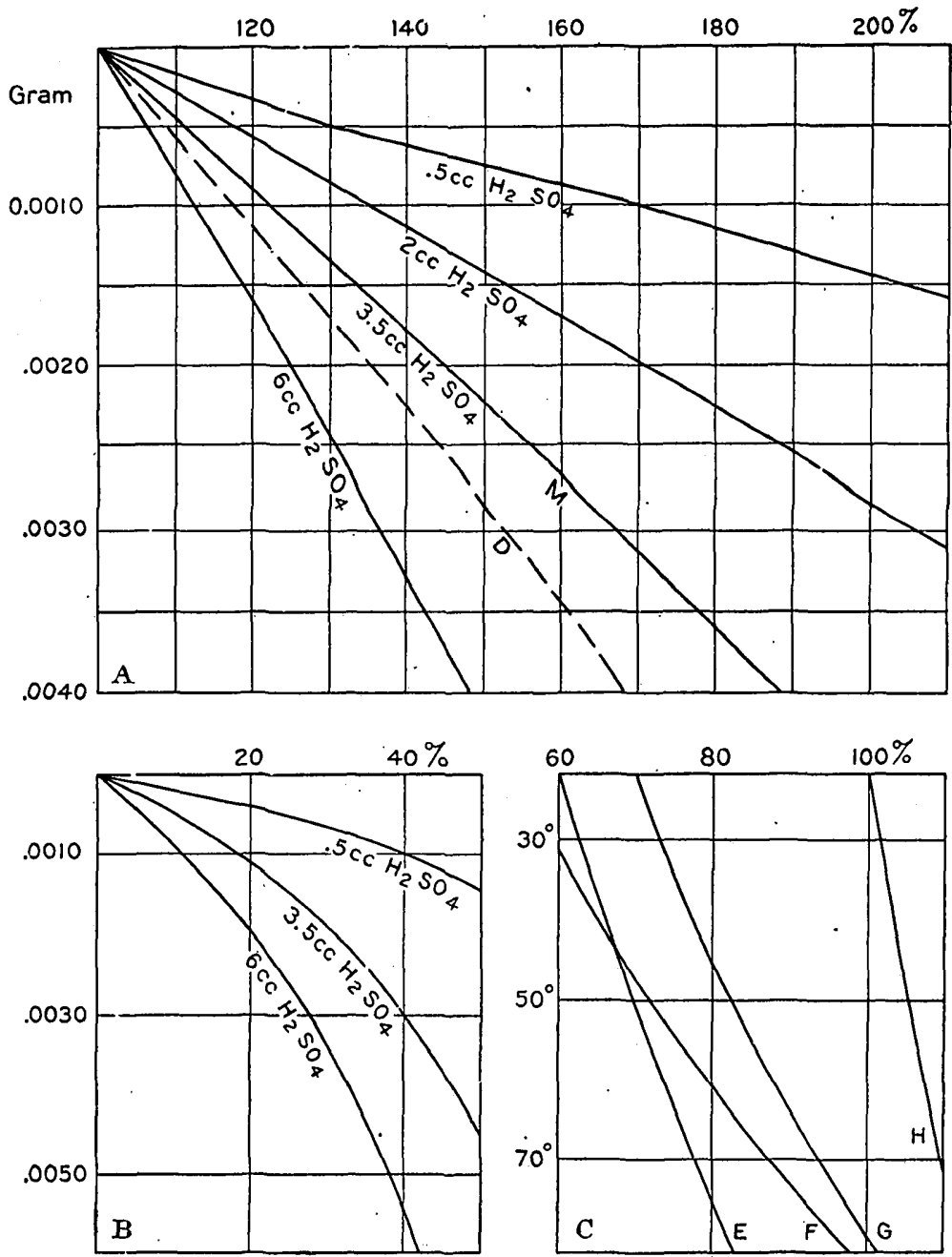

Fig. 27.-Merwin's diagrams showing the effect of fluorine with different concentrations of acid, and the effect of tempeiature. A. Coloration in peroxidized titanium solutions in presence of fluorine and free sulphuric acid. The fluorine in grams is read on the ordinate. The percentage ratios of depths of color in the standard solution to depths of color in the test solutions are read on the abscissa. For given amounts of fluorine and acid the ratio is found above the intersection of the lines representing the fluorine and acid. The curves are plotied for a temperature of $22^{\circ} \mathrm{C}$. The line $\mathrm{M}$ migrates to $\mathrm{D}$ as the solution is leated to $32^{\circ} \mathrm{C}$. B. The percentage of bleaching by fluorine may be found from these curves in the same way that the coloration ratios were found from the curves of $A$. C. Showing the rise in percentage of coloration due to heating. Curve $\mathrm{E}$ for a solution containing $1 \mathrm{~cm} .{ }^{8}$ of sulphuric acid and $0.0014 \mathrm{gram}$ of fluorine; $F$ for $3.5 \mathrm{~cm} .{ }^{8}$ of acid and $0.0037 \mathrm{gram}$ of fluorine; $G$ for $3.5 \mathrm{~cm} . .^{8}$ of acid and $0.0019 \mathrm{gram}$ of fluorine; $\mathrm{H}$ for a solution standard at $22^{\circ} \mathrm{C}$.

also the concentration of acid as well as the temperature affect the color of titanium solutions peroxidized by hydrogen peroxide. The 
salts cause bleaching, while addition of acid counteracts this effect to a considerable degree and increase of acid deepens the color. The effect of the alkali salts is opposed to that reported by Steiger (p. 190), but Merwin's statements have been confirmed by tests made at my instigation by Dr. William Blum at the Bureau of Standards. Steiger's observations as to the effect of other compounds are in general accord with those of Merwin. Blum further observed that with little acid present the color of the standard and of the bleached test can not be matched. This was so, for instance, with $3 \mathrm{mg}$. fluorine and 2 per cent sulphuric acid, but with 10 per cent acid a fairly satisfactory comparison was possible with as much as $5 \mathrm{mg}$. fluorine.

Merwin found in his study of the estimation of fluorine "that when the Nessler tube method was used the percentage ratios obtained by dividing the depth of each solution bleached by fluorine by the depth of its matched standard could be plotted in lines so nearly straight that from the lines simple formulas could be derived for use in analysis. The same ratios are obtained by dividing the final volume of the standard by the volume of the test in cases in which a colorimeter is used which requires the standard to be diluted."

The full lines of fig. 27, A, were thus plotted. Suppose this ratio $r$ in a particular case is 142 , and that there is $0.5 \mathrm{~cm} .^{3}$ of acid in the test solution, then the corresponding amount of fluorine is 0.0006 gram. The formula expressing this relation is $\frac{r-100}{70,000}=$ grams of fluorine. With $3.5 \mathrm{~cm}^{3}$ of acid in the test solution the formula is $\frac{r-100}{22,000}=$ grams of fluorine. By the conditions of the first formula amounts of fluorine between 0.00005 and 0.001 gram can be estimated accurately within 0.00005 gram, by the last formula amounts between 0.001 and 0.004 gram can be estimated within 0.00015 gram. By doubling the amounts of titanium and acid in the test solution and making it up to $100 \mathrm{~cm} .^{3}, 0.01$ gram of fluorine can be estimated.

The above formulas can not be used in rock analysis because of the disturbing effects of the alkali sulphates necessarily present in the test solution. In order to make it possible to know accurately the composition of the solution in which fluorine is to be determined in rock analysis, the following method has been worked out:

\section{B. Merwin's method:}

Two grams of rock powder are fused with 8 grams of mixed sodium and potassium carbonates and the fusion is taken up with hot water. When leached, and without the necessity for filtering, there are added 3 or 4 grams of powdered ammonium carbonate. The mixture is warmed for a few minutes, and then heated on the water bath till the ammonium carbonate is destroyed and the bulk of the liquid is small. In this way the silica, which otherwise might render the final solution turbid, is thrown down, together with the disturbing alumina and ferric oxide. The destruction of the ammonium carbonate is necessary because ammonium sulphate bleaches the final solution. After filtering there is added to the filtrate-which should not exceed $75 \mathrm{~cm}^{3}$ in volume -3 or $4 \mathrm{~cm} .^{3}$ of hydrogen peroxide, and then cautiously $10 \mathrm{~cm}^{3}$ of standard titanium solution ${ }^{a}$ (containing 0.01 gram $\mathrm{TiO}_{2}$ ). Including the acid in the titanium solution, about $4 \mathrm{~cm} .^{3}$ of strong sulphuric acid are

a The hydrogen peroxide prevents the precipitation of the titanium by the alkali carbonate. 
required to neutralize the alkali carbonates. As soon as neutrality is reached the solution acquires a light orange color. Neutrality is tested by adding a little sodium carbonate solution to discharge the color, and then a drop or two of acid to restore it. The further treatment depends upon the amount of fluorine expected. In the vast majority of cases this amount is less than 0.0025 gram $(0.125$ per cent of the sample). For such amounts there is added to the neutralized solution $3 \mathrm{~cm} \cdot{ }^{3}$ of concentrated sulphuric acid, and the solution is made up to $100 \mathrm{~cm}^{3}$. After being cooled to $22^{\circ} \mathrm{C}$. the solution is compared with a $100 \mathrm{~cm}^{3}$ solution containing 0.01 gram of $\mathrm{TiO}_{2}, 4 \mathrm{~cm} .{ }^{3}$ of $\mathrm{H}_{2} \mathrm{O}_{2}$, and 2 or $3 \mathrm{~cm}^{3}$ of concentrated sulphuric acid. The ratio, $r$, of depths (or volumes) of the solutions is obtained as described in the preceding section. This ratio is, however, much larger than the fluorine would give, owing to the alkali sulphates. The ratio that the alkali sulphates alone would give, if free from interfering substances, is about 125 . Different samples never give quite the same ratio. Those that give a ratio much higher than 125 probably contain fluorine. The safest way is to make determinations of this ratio on two 8-gram portions of the carbonates used in the fluorine estimation, $a$ and to use this ratio in making the correction. Having obtained this ratio-call it $m$-the formula for computing the fluorine is $\frac{r-m}{23,000}=$ grams of fluorine. Accuracy to 0.0002 gram may be expected. *** If the fluorine expected amounts to 0.0025 to $0.0120 \mathrm{gram}$, the test solution is made acid with $12 \mathrm{~cm} .^{3}$ of concentrated sulphuric acid, and compared as before described. The formula is $\frac{r-m-3}{6,300}=$ grams of fluorine; $m$ is to be determined and should not much exceed 108. Accuracy to 0.0005 gram may be expected.

It is very evident that the amounts of alkali salts and of acid must be taken into account and that the analyst should always take his readings at about the same temperature. For anyone who has frequent occasion to determine fluorine in this indirect way, it is desirable that he should prepare diagrams indicating the corrections to be made under his own conditions of operation.

\section{SULPHUR.}

\section{A. THE DETERMINATION OF SULPHUR AS BARIUM SULPHATE.}

a. THE ERRORS INVOLVED.

Owing to the small amounts of sulphur in rocks, special precautions in making the precipitation or special purification of the barium sulphate obtained as in $\mathrm{C}$ are hardly ever needful, especially as the precipitation is usually made in absence of iron. But when large amounts of sulphur are in question the case is very different. The causes of varying results by different chemists have given rise to many investigations and the end is not yet. Perhaps the most of these have had to do with the determination of sulphur in the commercially important pyrite, and it is now understood that only after solution of that mineral in aqua regia and removal of the iron by ammonia in a prescribed manner is it permissible to proceed to the

$a$ The 8 grams of carbonates dissolved in about $75 \mathrm{~cm} .^{\mathrm{a}}$ of water are treated precisely like the filtered fluorine solution.

20091-Bull. $422-10-13$ 
precipitation of the sulphur, and further that only when this is done under certain well-defined conditions is the result approximately correct, and then through compensation of considerable plus and minus errors. ${ }^{a}$

In rock and most mineral analyses the prior precipitation of interfering elements by ammonia will hardly ever be permissible, because there are almost invariably present others than iron, which, as, for instance, alumina, would tend to carry down the sulphate ion to a greater extent than does iron. Hence the conditions of the Lunge method will not be considered here, but chiefly those produced by leaching an alkali carbonate fusion or by direct solution in hydrochloric acid where this is permissible.

For the errors now known to arise under these conditions the following causes have been ascribed or tentatively suggested: Carrying down of barium chloride, of normal and acid sulphates of the alkalies, of complex salts like $\mathrm{BaCl} . \mathrm{HSO}_{4}(\mathrm{BaCl})_{2} \mathrm{SO}_{4},\left(\mathrm{M}^{\prime} \mathrm{SO}_{4}\right)_{2} \mathrm{Ba}$, $\left(\mathrm{HSO}_{4}\right)_{2} \mathrm{Ba}$. Of the above, $\mathrm{BaCl} . \mathrm{HSO}_{4}$ by itself would be presumably without influence on the final result after ignition of the precipitate. $\left(\mathrm{BaCl}_{2}\right)$ and $(\mathrm{BaCl})_{2} \mathrm{SO}_{4}$, singly or together, would occasion excessive weights. All others of the above-mentioned compounds would have an opposite effect, either because of the loss of sulphuric acid from acid salts (and of ammonium sulphate in special cases), or because of the low atomic weights of the alkali metals as compared with that of barium. The composition and concentration of the solution and the manner of precipitation will determine which of these undesirable products will contaminate the barium sulphate. For a given solution it is sometimes possible to regulate the conditions so as to reduce one or the other of the above to a minimum.

The presence of chlorine in barium sulphate precipitates was formerly ascribed solely to occlusion of barium chloride, but it seems to be now well established that this explanation can be only in part true; otherwise it would be difficult to account for the escape of hydrochloric acid on ignition. G. A. Hulett and L. H. Duschak have suggested ${ }^{b}$ as an explanation for this escape the existence of $\mathrm{HSO}_{4}{ }^{\prime}$ and $\mathrm{BaCl}^{\prime}$ ions in the solution and consequent formation of insoluble $\mathrm{BaCl} . \mathrm{HSO}_{4}$, which strong heat would decompose into $\mathrm{BaSO}_{4}$ and $\mathrm{HCl}$. But not all the chlorine can be thus removed, and the

$a$ The latest important contributions to this subject are those of $\mathrm{E}$. Hintz and F. Weber (Zeitschr. anal. Chemie, vol. 45, 1906, p. 31); G. Lunge and R. Stierlin (Zeitschr. angew. Chemie, vol. 18, 1905, p. 1921; Rept. Internat. Committee Appl. Chem. at Rome, 1906, p. 399); O. Folin (Jour. Biol. Chem., vol, 1, 1906, p. 131); and E. T. Allen and J. Johnston (as yet unpublished). The results of the most careful and elaborate investigation of the two last-named authors have been communicated to me as it progressed and I am indebted to them for some of the statements made in section A. The manuscript of Allen and Johnston was offered to me for perusal only as the correcting of the proof of this volume was ended, hence I am unable to cite their work as much as I would like. It embodies a great mass'of important quantitative data.

b Zeitschr. anorg. Chemie, vol. 40, 1904, p. 196. 
weight of the barium sulphate is still sometimes excessive. According to Hulett and Duschak this might be accounted for by the following ionic reactions:

$$
\begin{aligned}
& \text { 1. } 2 \mathrm{BaCl}+\mathrm{SO}_{4}=(\mathrm{BaCl})_{2} \mathrm{SO}_{4} \text {. } \\
& \text { 2. } 2 \mathrm{HSO}_{4}+\mathrm{Ba}=\left(\mathrm{HSO}_{4}\right)_{2} \mathrm{Ba} \text {. }
\end{aligned}
$$

By interaction of the resulting hypothetical salts when ignited together, either hydrochloric acid or sulphuric acid, or both, might be set free, according to the relative amounts of the respective salts. Notwithstanding the fact that Hulett and Duschak failed to find support for these reactions by proving the escape of sulphuric acid from their precipitates, Otto Folin has succeeded, ${ }^{a}$ as have also E. T. Allen and J. Johnston in the geophysical laboratory of the Carnegie Institution at Washington (private communication). The results may then, according to the conditions of precipitation, be too high or too low. In further explanation of too high results Folin suggests that, in presence of soluble sulphates of univalent metals, $\mathrm{MSO}_{4}^{\prime}$ ions may take part in the reactions, yielding $\left(\mathrm{MSO}_{4}\right)_{2} \mathrm{Ba}$, which, as well as $(\mathrm{BaCl})_{2} \mathrm{SO}_{4}$, should be stable on moderate ignition. That alkalies in the form of stable sulphates (assumed normal) do form part of the precipitate of barium sulphate that is formed in alkali chloride solutions, Hintz and Weber ${ }^{b}$ have pointed out, and this Allen and Johnston have conclusively and quantitatively proved by direct determination. The extent of such contamination has been so great as to give the last-named authors losses of 0.5 per cent or more of the total sulphur in artificial mineral sulphides of ideal composition. This discovery did not, however, account for all the deficit regularly observed by them. The balance was found to be due to loss of sulphuric acid on ignition of the precipitate. Lunge and Stièrlin, in the paper already cited, also made correction for normal alkali sulphates, but, so far as can be judged, without direct determination.

Other sources of error are due to solvent action of hydrochloric acid and of alkali salts in the solution. ${ }^{c}$ For these it is not difficult, ordinarily, to correct by appropriate treatment of the filtrates. Still others, mentioned by I. Pattinson and J. T. Dunn, ${ }^{d}$ may arise from the presence of sulphides in some barium chloride and from red rubber stoppers. Finally, there is the possibility of reduction to barium sulphide if the precipitate is ignited in contact with paper.

b. AVOIDANCE OR CORRECTION OF ERRORS.

It is thus apparent that the accurate determination of the sulphate ion is one subject to many varying errors and that the methods of procedure and correcting for impurities must be varied to suit the

a Jour. Biol. Chem., vol. 1, 1906, p. 131.

$b$ Loc. cit.

c Allen and Johnston seem to have shown that alkali salts are practically without effect in this respect.

a Jour. Soc. Chem. Ind., vol. 29, 1905, p. 10; Chem. Zeitung, vol. 29, 1905, p. 29. 
conditions. General directions to meet all conditions can not be given. Hintz and Weber ${ }^{a}$ obtain with pyrite, after separation of the iron, correct weights by adding the precipitant all at once in order to offset the effect of normal or acid alkali sulphates by increase of occluded barium chloride. Allowable and satisfactory as this may be for commercial analysis, when results of the highest accuracy are sought the errors must be each and every one measured. The results are then more concordant than by the method of Hintz and Weber (Allen and Johnston).

When pure sulphuric acid is precipitated by barium chloride, the problem is relatively simple because of the absence of soluble salts; hence in mineral analysis it is desirable to avoid an alkali fusion whenever possible, but it must not be overlooked that direct treatment of the mineral with hydrochloric acid or oxidizing reagents involves the presence of certain amounts of salts, one or another of which may be fatal to the attainment of acceptable results.

In general, there should be present the least amount of free hydrochloric acid that will prevent hydrolysis of salts in the hot solution, since the acid has marked solvent effect on barium sulphate. ${ }^{b}$ Nitric acid should be avoided or removed by evaporation with hydrochloric acid. The barium chloride solution used as precipitant should be of not over 10 per cent strength and should be delivered slowly, in fine drops, preferably from a capillary tube, into the nearly boiling test solution. The precipitate will then be well crystallized and will contain a minimum and sometimes entirely negligible amount of chlorine, whereas with rapid precipitation its amount may be several milligrams per gram of precipitate.

The chlorine in the ignited precipitate may be determined and deducted (if known to be present as such) from the gross weight. Hulett and Duschak ${ }^{c}$ dissolve the ignited and weighed sulphate in concentrated sulphuric acid at $100^{\circ}$ while a current of air carries the freed hydrochloric acid over into a dilute solution of silver nitrate.

If contaminating alkali is to be determined, a paper filter should be used for the collection of the precipitate. In the latter case the paper should be charred very slowly and without any active combustion, otherwise there may be marked reduction of the sulphate to sulphide (E. T. Allen). After ignition the precipitate is to be dissolved in the least amount of hot concentrated sulphuric acid, the resulting solution is to be poured into cold water, heated for some time, filtered, the filtrate evaporated in platinum, the excess of acid expelled,

$a$ Loc. cit.

$b$ With alkali sulphates Allen and Johnston get the best results when the solution contains only $0.1 \mathrm{~cm} .{ }^{3}$. of concentracted $\mathrm{HCl}$ in volume of $350 \mathrm{~cm} .^{3}$.

c Zeitschr. anorg. Chemie, vol. 40. 1904, p. 106. 
and the residue of alkali sulphate weighed and tested as to purity. Its amount is to be increased by 10 per cent for that still retained by the barium sulphate (Allen and Johnston).

Lioss of sulphuric acid by decomposition of an acid salt or volatilization of ammonium sulphate can be measured, so far as known at present, only by conducting the ignition so as to permit the collection of the escaping gases. Since these gases may contain sulphur dioxide and oxygen, as well as sulphur trioxide, they should be passed through a solution of bromine or hydrogen peroxide. For this determination a perforated filter of deep and narrow form should be used, which can be inserted into the decomposition apparatus and weighed after the ignition is over.

The application of all three of these corrections can seldom be possible, for as a rule each of them calls for a separate portion of a very large precipitate, or else there must be available three different large precipitates with exactly the same amounts of contamination, a condition difficult if not impossible to secure. The determination of fixed chlorine and volatile sulphuric acid might, however, be made with one and the same portion, the volatile sulphur being first determined and afterwards the chlorine. It is to be borne in mind, however, that, as shown by Hulett and Duschak, the so-called fixed chlorine itself begins to escape above $700^{\circ}$, the residue then showing alkalinity.

Errors due to the solvent action of hydrochloric acid or of ammonium or fixed salts in the solution can usually be corrected by evaporating the filtrates from the barium sulphate, destroying or expelling ammonium salts if present, treating the residue with water slightly acidified with hydrochloric acid, and collecting and purifying the barium. sulphate that may be left undissolved.

\section{B. DETERMINATION OF THE CONDITION OF SULPHUR IN ROCKS.}

Before proceeding to the determination of sulphur, its condition, if present, should be ascertained.

Evolution of hydrogen sulphide on boiling with diluted hydrochloric acid is evidence of a soluble sulphide, usually pyrrhotite, but possibly lazurite. Extraction of magnetic particles reacting for sulphur shows pyrrhotite to have been in part at least the source of the hydrogen sulphide. A reaction for sulphuric acid in the filtered solution indicates a soluble sulphate, usually a silicate sulphate, noselite, or hauynite. If the residue, when well washed and treated with aqua regia or hydrochloric acid and bromine, gives more sulphuric acid, the probable presence of pyrite is shown. Should this solution likewise show arsenic, the sulphide may be arsenopyrite, which, however, is of very rare occurrence in igneous rocks, if, indeed, it is ever found there. 
It is most uncommon to find any sulphur in rocks in the ordinary simple sulphate condition, except sometimes water-soluble traces, even when considerable pyrite is in evidence. The barium of fresh and even moderately decomposed rocks is apparently always in silicates and not in combination with sulphur.

\section{QUANTITATIVE DETERMINATION.}

a. TOtal SUlphur.

Although the sulphur of sulphides may sometimes be wholly extracted with aqua regia or some other powerful oxidizer, in the wet way, this is not always so. Therefore it is generally better by far to fuse with sulphur-free sodium carbonate and a little niter over the Bunsen burner, and for a few moments over the blast, using throughout the heating the arrangement shown in fig. 3 (p. 32), in order to exclude access of sulphur from the flame gases. This determination may very conveniently be combined with those of barium, titanium, zirconium, and rare earths (p. 138). After thorough disintegration of the fusion in water, to which a drop or two of alcohol has been added for the purpose of reducing and precipitating any manganese dissolved as manganate, the solution is filtered and the residue washed with a dilute solution of sodium carbonate. In the filtrate $\left(100\right.$ to $250 \mathrm{~cm}^{3}$ in bulk) the sulphur is precipitated at boiling heat or on the steam bath by barium chloride in excess, after slightly acidifying in the cold by hydrochloric acid. Evaporation to dryness first with acid, in order to eliminate silica, is needless, for in the above bulk of solution there will almost never be the least separation of silica with the barium sulphate. $^{a}$ It is well that this is so, for evaporation on a bath heated by gas to remove silica would in many cases involve an error fully equal to the sulphur present by contamination from the sulphur of the gas burned. With a steam bath this difficulty does not present itself. Should there be fear of a trace of silica being present, it can be removed by a drop of hydrofluoric and sulphuric acids before weighing the barium sulphate.

If the amount of precipitate is large enough to permit and the circumstances demand, other corrections should be applied before ignition, as mentioned under A. b, page 195 .

\section{b. FRACTIONAL DETERMINATIONS.}

If traces of water-soluble sulphates are present, they can be removed when desired by boiling water.

$a$ The results of C. $\dot{W}$. Stoddart (Jour. Am. Chem. Soc., vol. 24, 1902, p. 852) show, in the determination of sulphur in coals by the Eschka method, often higher sulphur when silica is not removed by evaporation in the usual way, but this is contrary to all my experience in silicate work, where duplicates agree usually exactly, and if they differ are as likely to be high by one way as by the other. The explanation may possibly be that Stoddart acidified his solutions hot or used too strong an acid and had some silica precipitated. 
For the quantitative extraction of the sulphur of all soluble sulphates, simple boiling with hydrochloric acid suffices, which should be done in an atmosphere of carbonic acid if pyrites or other oxidizable sulphides are present, and should be finished as quickly as possible in crder to minimize the error resulting from oxidation to sulphuric acid of the sulphur of sulphides, if present, by any ferric salts that may have been dissolved.

If soluble sulphates and sulphides as well as insoluble sulphates and sulphides are present together, the sulphur of the first is found in solution after extraction by hydrochloric acid in a carbon-dioxide atmosphere, and that of the decomposable sulphides by collecting the hydrogen sulphide evolved. ${ }^{a}$ In the residue the sulphur of the insoluble sulphides can be estimated, or from the total sulphur found in another portion its amount can be calculated. The error involved in the above estimation of the sulphur of soluble sulphides, due to the possible reducing effect of hydrogen sulphide on ferric salts, is probably negligible. Most of the hydrogen sulphide would be expelled before any such action could take place and probably before the ferric salts were largely attacked, but of course the small proportion of sulphur set free as such from pyrrhotite would escape estimation and introduce further uncertainty. In general, it would be safe enough to assume the composition $\mathrm{Fe}_{7} \mathrm{~S}_{8}$ for pyrrhotite. However carefully all. these separate determinations may be carried out, the final figures for ferrous and ferric oxides can hardly be regarded as more than approximations when much sulphide is present (see c. $\alpha$, p. 165).

\section{BORON.}

No doubt boron occurs occasionally in rocks in amounts superior to those of some of the other constituents that are habitually determined. But the lack of precision of the methods that are at all applicable to mineral substances not directly soluble in acids other than hydrofluoric acid is accountable for the fact that no one has ever sought to apply these methods to rocks, unless boron was supposed to be present in appreciable amount. When this condition is fulfilled it is customary to fuse with an alkali carbonate and then apply in one of its numerous modifications the Rosenbladt-Gooch method. In the footnote below ${ }^{b}$ are given the most important

\footnotetext{
a With pyrrhotite a small fraction of its sulphur-one-eighth if the formula $\mathrm{Fe}_{7} \mathrm{~S}_{8}$ is adopted-is liberated as free sulphur and not as hydrogen sulphide.

$b$ Th. Rosenbladt (Zeitschr. anal. Chemie, vol. 26, 1887, p. 21) used magnesia for binding the boron, while F. A. Gooch (Proc. Am. Acad. Arts Sci., 1886, p. 167; Bull. U. S. Geol. Survey No. 42, 1887, p. 64; Chem. News, vol. 55,1887, p. 7) preferred lime, as more active and reliable. Gooch and L. C. Jones have later (Am. Jour. Sci., 4th ser., vol. 7, 1899, p. 34; Chem. News, vol. 79, 1899, pp. 99, 1.11) upheld the use of lime and proposed as a convenient though perhaps not quite so perfect substitute, sodium tungstate containing an excess of tungstic oxide. In this article they likewise indicate the precautions now used to insure complete collection and retention of the boron.

For a useful modification in the way of collecting the boric ether in ammonia before bringing in contact with the lime, see S. L. Penfield and E. S. Sperry (Am. Jour. Sci., 3d ser., vol. 34, 1887, p. 222); also
} 
references that relate to the development of this method and its application to refractory minerals.

The method depends on expelling the boron as methyl ester by distillation with methyl alcohol from an acid solution, collecting the ester in some suitable manner and then determining the boron gravimetrically or volumetrically. For simple borates, especially the soluble ones, the method gives satisfaction when all needful precautions are carefully observed, and that it may do so also with refractory borosilicates has been shown by several workers, most recently by Chapin. Before Chapin it was considered necessary to extract with water the alkaline fusion of a mineral and to treat the extract for the boron. A cause for the wide divergence of results obtained by different chemists, even between duplicate results by the same analyst, is probably referable to the employment of only a single fusion and extraction, for it has been shown by S. L. Penfield and H. W. Foote ${ }^{a}$, by G. W. Sargent ${ }^{b}$, and by W. E. Ford ${ }^{c}$ that these operations must be repeated. A decided advance seems to have been scored by Chapin, who shows that aluminum and iron need not be removed, but that the carbonate melt may be dissolved directly in acid without prejudice to the accuracy of the method as carried out by him. Furthermore, the presence of chlorine, even of fluorine, does not seem to be detrimental. Possibly his procedure may yet prove applicable to rocks as well as to boron minerals. It is, however, a method which depends on the titration of the boron in the distillate, and is hence less satisfactory than one which permits weighing the boron and then making sure of its presence by a qualitative test.

E. T. Wherry ${ }^{d}$ has devised a method for refractory boron minerals that yielded him results comparing favorably with those of Chapin, the same samples being used. It depends on separating aluminum and iron by means of calcium carbonate in a special manner, after making an alkali fusion, and titrating the filtrate directly in presence of mannite. The aluminum and iron must, however, be redissolved and reprecipitated one or more times.

H. Moissan (Compt. Rend., vol. 116, 1893, p. 1087; Bull. Soc. chim., vol. 12, 1894, p. 955), who modifies the Gooch distilling apparatus in certain respects.

G. W. Sargent (Jour. Am. Chem. Soc., vol. 21, 1899, p. 858) obtained satisfactory results with tourmaline by collecting the boron in sodium-hydroxide solution, neutralizing the alkali with hydrochloric acid by the aid of methyl crange, then titrating the freed boric acid by sodium hydroxide in presence of glycerin, using phenolphthalein as indicator (method of Thompson, Jour. Soc. Chem. Ind., vol. 12, 1893, p. 432).

The latest contributions to the application of this method are by R. J. Manning and W. R. Lang (Jour. Soc. Chem. Ind., vol. 25, 1906, p. 396), who determine the boron in the distillate gravimetrically as the barium salt $\mathrm{Ba}\left(\mathrm{BO}_{2}\right)_{2}$, and by Wilson H. Low (Jour. Am. Chem. Soc., vol. 28, 1906, p. 807), who modifies the distillation feature, with much apparent advantage, by the addition of dry granulated calcium chloride and perfects the turmeric-paper qualitative test. This author titrates the distillate in presence of glycerin. W. H. Chapin (Jour. Am. Chem. Soc., vol. 30, 1908, p. 1691) applies the Low method successfully witb slight modifications to borosilicates, but prefers mannite to glycerin.

a Am. Jour. Sci., 4th ser., vol. 7, 1899; p. 97.

b Jour. Am. Chem. Soc., vol. 21, 1899, p. 858.

c Am. Jour. Sci., 4th ser., vol. 14, 1903, p. 195.

d Jour. Am. Chem. Soc., vo!. 30, 1908, p. 1687. 
If, as sumetimes may happen, the problem is presented of examining rocks for traces of gold, silver, and other elements which are not ordinarily looked for, as in F. Sandberger's investigations bearing on the origin of the metalliferous contents of veins, large weights of material must be taken, up to 50 grams or more. This involves the use, also, of large quantities of reagents, the purity of which must then be looked to with the utmost care. Special directions to meet such cases can not now be given, nor even a complete reference list of the scanty and scattered literature on this subject. Sandberger's own writings deal but little with its analytical side, and from its inaccessibility in the Washington libraries I am as yet unacquainted with the report by $\mathrm{H}$. Von Foullon "Uèber den Gang und die Ausführung der chemischen Untersuchung," following Sandberger's own paper ${ }^{b}$ in the general report "Untersuchungen der Nebengesteine der Pribramer Gänge." I have published a few data as to gold, silver, lead, zinc, etc., ${ }^{c}$ in S. F. Emmons's report on "The geology and mining industry of Leadville;" J. S. Curtis, ${ }^{d}$ in his report on "The silverlead deposits of Eureka, Nev.," has given his method of assaying rocks for traces of gold and silver; in volume 7 of the Reports of the Missouri Geological Survey, page 740, are to be found the methods used by James D. Robertson for the determination of lead, zinc, and copper in silicate and carbonate rocks of Missouri. The earlier work of J. G. Forchhammer ${ }^{e}$ and L. Dieulafait, ${ }^{f}$ the latter of whom examined many hundreds of Archean rocks, was of a qualitative nature.

\section{THE GASES AND VAPORS EXPELLED BY HEAT.}

That rocks and minerals evolve large quantities of gases and vapors when heated is a well-recognized fact. Numerous analyses have shown that the volatile products usually comprise hydrogen and carbon dioxide as preponderating constituents, with carbon monoxide, methane, nitrogen, hydrogen sulphide, etc., in smaller amounts. The total volume may be many times that of the solid. To a certain extent, sometimes a large extent, especially as to carbon dioxide, the source of these is entrapped fluid inclusions, whose origin was contemporaneous with that of the containing minerals. A further portion

a Jahrb. der Bergakad. Leoben u. Pribram, 1887, p. 363.

$b$ From Sandberger's report it appears that the rocks were treated successively with water, acetic acid, boiling dilute hydrochloric acid for two days, and finally hydrofluoric acid, the several extracts and final residue of fluorides (and pyrite) being separately examined for heavy metals. The products of distillation were also examined. A striking fact observed in all cases was the complete insolubility of the pyrite, even after the severe treatment mentioned. This speaks strongly in favor of the correctness of ferrous iron estimations in silicates by the hydrofluoric and sulphuric acid method when pyrite is present unaccompanied by other sulphides. (See $a$, p. 165.)

c Mon. U. S. Geol. Survey, vol. 12, 1886, Appendix B, pp. 592-596.

$d$ Idem, vol. 7, 1884, pp. 120-138.

e Pogg. Ann., vol. 95, 1855, p. 60 .

$f$ Annales chim. phys., vols. $15,17,18,21,1878-1880$. 
may have been held dissolved in the rock, particularly in those not wholly crystallized. But from the experiments of some recent investigators, especially Morris W. Travers, ${ }^{a}$ A. Gautier, ${ }^{b}$ and K. Hüttner, ${ }^{c}$ it is very evident that the gases are often produced by chemical changes resulting from the heat used for their expulsion. 'Thus, hydrogen may be due to reduction of water by minerals susceptible of oxidation at high temperatures, as ferrous silicates; the carbon monoxide to reduction of carbon dioxide in a like manner or by the hydrogen formed by the first reaction; the methane might arise from interaction between water and traces of metallic carbides, etc. ${ }^{d}$

Free oxygen is not reported as one of the evolved gases, and from the nature of the case it should hardly be expected.

Nitrogen, though found in but small relative amount in the free state, is comparatively abundant and easily detectable as ammonia or ammonium salts in certain classes of rocks and was long since thus recognized. H. Rose ${ }^{e}$ says that pitchstone gives off ammoniacal water on heating; A. Delesse ${ }^{f}$ found ammonia in rocks, and A. Gautier later made quantitative tests; H. Erdmann ${ }^{g}$ found that ammonia was obtained by acting on various minerals of ancient igneous rocks with a caustic alkali; C. Luedeking and H. A. Wheeler ${ }^{h}$ found ammonium sulphate in a barite from Missouri, the presence of which I was able to confirm.

It has been noted in this laboratory on three separate occasions, when series of ores, roofing slates, and eruptive rọcks were analyzed, that ammonia, either in the form of chloride or sulphate, or even as free ammonia, was given off on heating. Its appearance was not limited to one or a few specimens of a series, but seemed to be characteristic of all and to be afforded by the unbroken rock as well as by the powdered sample. The precise conditions under which the specimens were collected not being known, it is impossible to affirm positively that the ammonia may not have been due to recent organic contamination of some sort, especially in the case of the slates, but it is believed that a more critical collection of material will not alter the general result. Its amount was sometimes readily determinable by Nesslerization, being as high as 0.04 per cent in some slates. Carbonaceous organic matter was absent from most of these, but doubtless existed in them in their early history. In their case the ammonia was, in part at least, evolved as such, imparting a strong alkaline reaction to the

\footnotetext{
a Proc. Roy. Soc., vol. 64,1899 , p. 130.

$b$ Compt. Rend., vol. 131, 1900, p. 647; vol. 132, 1901, pp. 58 and 189; vol. 136, 1903, p. 16; Annales chim. phys. , 7th ser., vol. 22, 1901, p. 97; Annales des Mines, 10th ser., vol. 9, 1906, p. 316.

c Zeitschr. anorg. Chemie, vol. 43, 1905, p. 8 .

$d$ For the methods of collecting and analyzing such gaseous mixtures consult Travers, M. W., The experimental study of gases; the above-cited papers of Gautier; and Moureu, Charles, Compt. Rend., vol. 142, 1906, p. 44.

$e$ Quantitative Analyse, Finkener ed., p. 673.

$f$ Annales des mines, vol. 18, 1860, p. 151.

$g$ Ber. Deutsch. chem. Gesell., vol. 29, 1896, p. 1710.

h Am. Jour. Scl., 3d ser., vol. 42, 1891, p. 495.
} 
water in the upper part of the tube. The presence of sulphides, fluorides, or chlorides in the rock might cause the ammonia to appear as a sublimate of sulphate, fluoride, or chloride. It has been suggested that ammonia might result from the action of water on metallic nitrides, O. Silvestri ${ }^{a}$ having observed a nitride of iron on a lava from Etna.

\section{SPECIAL OPERATIONS.}

The problem often presents itself of ascertaining the composition of that portion of a rock powder which is soluble in special reagents or in a reagent of a particular concentration. No precise directions can be formulated to meet such cases. The procedure must vary with the character of the constituents of the rock and with the object which it is sought to attain, and only in exceptional cases can a separation of this kind be sharp. Much depends on the degree of fineness of the powder and on the length of time it is exposed to the action of the reagent.

\section{A. DETECTION OF NEPHELINE IN PRESENCE OF OLIVINE. .}

For confirmation of the microscopic diagnosis, Prof. L. V. Pirsson ${ }^{b}$ has indicated a means of detecting nepheline in presence of olivine, as in nepheline basalts, based on the very ready solubility of nepheline, as compared with olivine, when boiled for but one minute with a sufficiency of very dilute nitric acid (1:40). Gelatinization of the filtrate on evaporation is taken as evidence of the presence of nepheline. If olivine is present in quantity, however, this test must not be accepted at once as final, for some, if not all, olivines are much more soluble in nitric acid of the above strength than Professor Pirsson was led to believe from his original tests. If, therefore, on evaporation of the filtrate, much iron is indicated, the gelatinization may well be due to olivine alone or in part, and then the quantitative relation of silica to iron plus magnesium should be ascertained. It must also be borne in mind that any other very soluble silicates present will be more or less affected, and that apatite is largely or wholly dissolved. It is possible that still more dilute nitric or perhaps some other acid may exert a slighter solvent action on olivine without being appreciably less effective in dissolving nepheline, etc. In combination with a quantitative analysis of the extract the method is perhaps susceptible of a wider application than the particular case for which it was first used. . It is · well worth further study.

\section{B. DETERMINATION OF SOLUBLE SILICA.}

Very often in treatment by acids silica is separated in gelatinous or granular form mixed with the unattacked minerals, and it becomes necessary to remove or estimate this silica, or else to discrimi- 
nate between soluble and insoluble silica already existing together. Usually a boiling solution of sodium carbonate has been employed for this purpose, though the caustic alkalies have found advocates.

G. Lunge and C. Millberg ${ }^{a}$ have conclusively shown that quartz is not nearly so insoluble in solutions of the caustic alkalies as has been supposed, but that given a sufficient degree of subdivision it can be brought wholly into solution; that it is impossible to secure correct separation of quartz and opaline silica by the use of either caustic or carbonated alkalies; and that digestion on the water bath for fifteen minutes with 5 per cent solution of sodium carbonate is the only way to secure exact separation of unignited precipitated silica from quartz, and then only when the finest flour has been removed by levigation. The authors say:

If, however, no more of such flour is present than is produced in the ordinary operations of powdering and sifting through cloth of the finest mesh, the error arising from the above-mentioned treatment is so slight that it can generally be neglected; it reaches 0.1 to, at the most, 0.2 per cent of the total silica, by which amount the quartz will appear too low, the amorphous silica too high.

The above authors also show, however, that the solvent action of the caustic alkalies on quartz becomes very apparent only when the material has been reduced to such an utterly impalpable degree of fineness as is practically never reached in the preparation of samples for rock analysis. For this reason I have no hesitation in recommending the employment of a dilute solution of sodium hydroxide when the silica separated by acid from one of several mineral constituents of a rock is to be estimated. Even when dilution is considerable, solution is almost immediate, and as soon as this is accomplished-the point being known by the change in appearance of the residue-the solution should be diluted with cold water and filtered at once. The difficulty met with in filtration may often be overcome by faintly acidifying, which has the added advantage of at once arresting any further action of the alkali. If the dilution is sufficient no separation of silica results from so doing. Very dilute acid should also be used for washing. Lunge, when using sodium carbonate, washes with hot carbonate solution to which alcohol has been added, thus obtaining clear filtrates.

B. Sjollema ${ }^{b}$ regards diethylamine as suitable for the separation of amorphous silica from quartz and silicates in soils. He found that a 33 per cent boiling aqueous solution of the reagent dissolved 0.65 gram of amorphous silica practically wholly after eight hours' action and a 16 per cent solution within 1 per cent, whereas the solubility of quartz powder was only 1.2 per cent after two days.

a Zeitschr. angew. Chemie, 1897, pp. 393, 425.

a Jour. landw. Chemie, vol. 50, 1902, p. 371; abstracts in Jour. Chem. Soc., vol. 84, 1903, p. 241; Analyst, vol. 28, 1903, p. 123; Chem. Zeit., Rep., 1903, p. 21. 


\section{CARBONATE ROCK ANALYSIS.}

\section{PART I.-INTRODUCTION.}

29. QUALITATIVE COMPARISON OF CARBONATE AND SILICATE ROCKS.

The analysis of an ideally pure carbonate of any one metal is of great simplicity. Occasionally the carbonates met with in nature approach but probably never fully reach this condition of purity, as when we find them in the form of well-crystallized mineral species, like calcite, magnesite, siderite, cerussite, etc. Even in such cases it is usually true that the crystals either contain included foreign matter to a slight extent or are composed of more than one carbonate in isomorphous mixture, and then the separation of the different metals may demand considerable skill and time. The great carbonate formations of the earth's crust are never of this simple character, however, even the most dazzling white marble contains other ingredients than calcium carbonate, and the degree of admixture may vary from almost nothing up to that indefinite point beyond which the rocks cease to be classified as limestones or dolomites, but rather as calcareous shales, sandstones, etc.

The matters other than carbonates which these rocks may contain are in the main original constituents, having been introduced at the time the carbonates were themselves deposited. They may consist of the detritus of earlier geologic formations in the form of sand or clay that has undergone little or no alteration in the subsequent passage of time, besides carbonaceous matter derived from the marine life existing during their formation. But besides these there may be found secondary minerals that have resulted from chemical transformations in the mass of the accumulated sediments, either with or without the powerful aid of dynamic forces like long-continued movements of the crust or the intrusion of hot igneous rocks. The metamorphism produced by these last agencies is often of a most profound character and may result in the production of a great variety of minerals altogether foreign to the original sediments. The metamorphism may have been accompanied by the introduction and incorporation of new material, but even in the contrary case all the chemical constituents of the original detrital foreign matter have necessarily become a part of the carbonate rocks. These constituents are furthermore necessarily those common to silicate rocks of the earth's crust, hence the carbonate rocks differ qualitatively in no essential respect from their silicate associates except as to the more common occurrence of carbonaceous 
matter; and a complete analysis of one of them involves most of the determinations and separations described in the first part of this work. The analytical procedure differs, however, in some respects, owing to the great preponderance in most cases of components easily soluble in the common mineral acids, which either renders it unnecessary to use an alkaline flux or enables the analyst to reduce its amount very materially, thereby simplifying subsequent operations. The analysis is further simplified by the occurrence in reduced amounts, as a rule, of those elements which most complicate the analysis of a silicate rock. Only in the very purest carbonate rocks are some of them nearly or entirely lacking, as silicon, aluminum, and the alkalies, but the minor constituents zirconium, barium, and strontium are often not present in readily determinable amount.

In the following pages the descriptions will apply especially to those large deposits of carbonate materials denominated limestones, magnesian or dolomitic limestones, and dolomites, many of which are of great and growing economic importance. For the purpose of the analyst an exact definition of these terms is of minor significance. Indeed, among geologists there has been no uniform rule for their delimitation, and from the necessity of the case any rule must be purely arbitrary, since there is absolutely no break in the series between limestones and dolomites. The last name strictly applies only to the equiatomic double salt of calcium and magnesium, $\mathrm{CaMg}\left(\mathrm{CO}_{3}\right)_{2}$, and by some it is held that this should be its only application. On the other hand, the opposite extreme has been approached in contending that all limestones with as much as 1 or 2 per cent of magnesium carbonate should be classed as dolomites. According to H. Ries ${ }^{a}$. the most prevalent practice among geologists seems to be to call all limestones with from 5 to 18 per cent of magnesium carbonate magnesian limestones and the rest limestones or dolomites, according as their magnesium-carbonate contents lie below or above these limits. Some, however, while subscribing to the above definition of magnesian limestones, would and do call those rocks with from 20 per cent of magnesium carbonate up to the dolomitic ratio dolomitic limestones. F. W. Pfaff ${ }^{b}$ calls limestones with 11 to 46 per cent of $\mathrm{MgCO}_{3}$ dolomites, since these are common, while those with 7 to 11 per cent he says are very rare, if indeed they occur at all.

It has seldom been considered necessary to analyze the carbonate rocks with any great attention to detail. The majority of analyses have been made with reference to their technical utilization and with determination of only the more important constituents. In such cases it has been a common practice to omit direct determination of the carbon dioxide and either to calculate its supposed amount on 
the basis of the total lime and magnesia found or to regard the loss on ignition as representing the carbon dioxide. The portion insoluble in hydrochloric acid has been frequently stated as silica. Any iron that may have been determined has been reported as $\mathrm{Fe}_{2} \mathrm{O}_{3}$, water and carbonaceous matter have been entirely overlooked or neglected, as also titanium, phosphorus, and the rarer constituents, and sulphur has been almost uniformly reported as $\mathrm{SO}_{3}$. Such practice has repeatedly involved very gross errors of omission as well as of commission, which, though of little moment in many cases from the commercial point of view, detract much from the value of all these analyses in the eyes of the geologist intent on gaining an exact knowledge of an important formation and of the possible sources of metallic ore deposits of economic value, whether these last are residual soils resulting from the subaerial decomposition of limestone formations or whether they are deposits of the ores of lead, zinc, vanadium, etc., that have been formed beneath the surface by the agency of circulating waters.

\section{MINERAL COMPOSITION OF CARBONATE ROCKS.}

The chief mineral compounds entering into the make-up of the carbonate rocks are of course calcium carbonate for the limestones proper, with the double carbonate, the mineral dolomite, in increasing proportions through the magnesian limestones and dolomites; but it is the exception to find them free from other carbonates as essential components, chiefly those of iron and manganese, which are usually in isomorphous combination with the constituents of one or the other of the above minerals. The proportion of these is far less than that of the others, but sometimes aggregates several per cent. When they predominate the rock is no longer a limestone, but becomes an ore of iron or manganese.

Neglect to take account of these minor carbonate constituents is a frequent source of error in the statement of analyses, where it is very common to find reported as carbonates only calcium and magnesium, and perhaps a considerable amount of iron as $\mathrm{Fe}_{2} \mathrm{O}_{3}$ and manganese as one of its higher oxides, although it is practically certain that these must have existed in large part as ferrous and manganous carbonate. A careful determination of the carbon dioxide will often tell whether this is so or not, for it will perhaps be found in excess of that required for lime and magnesia. A negative result, however, is not to be taken as proof of the absence of iron or manganese carbonate or both, for very often a small portion of the magnesia may be in silicate combination, or in some formations there may be a little gypsum to claim a portion of the lime. This last is not likely to be the case in the average hard rock that is used for the manufacture of cement. In such the sulphur often reported as $\mathrm{SO}_{3}$ is likely to come from iron pyrites and perhaps to a limited extent at 
times from carbonaceous matter. It is hence easy to see that except in absence of iron and manganese it is not possible to ascertain the exact proportions of calcium and magnesium carbonates, and in the excepted cases it is sometimes possible only after separate analyses of the parts soluble and insoluble in dilute acid have been made.

The commonest sulphide in limestone formations is pyrite, though its presence if often obscured by its extremely fine state of division or by carbonaceous matter. In the metamorphosed limestones, especially those altered by intruded igneous bodies, other sulphides, as sphalerite and galena, are not uncommon, and these and still other sulphides may form important ore bodies at times or again give rise by oxidation to equally or even more valuable ores of a different sort. The strongly metamorphosed siliceous limestones are, however, seldom subjected to chemical analysis, especially for commercial purposes.

The siliceous components may be of the most varied character. Their names alone might fill a page. Suffice it to mention for the metamorphosed rocks garnet, vesuvianite, wollastonite, tremolite, diopside, the scapolites, tourmaline, apatite, and silica in the form of chert. Cherty limestones are extremely common and their silica was doubtless introduced during metamorphism. The chief siliceous components of unmetamorphosed limestone rocks are, as said before, sand and a clay. The latter particularly, since it is a clay, carries water, and this fact is usually overlooked in ordinary analyses. The analytical statements may show no water, though clayey matter is present to a large extent. The sand may be largely or altogether. quartz, or it may be composed in part of such other minerals as are commonly found in sands, like mica, garnet, zircon, etc. These latter may be often detected, even in the less siliceous and clayey limestones, by dissolving large amounts in hydrochloric or acetic acid and subjecting the residue to microscopic examination, after burning off obscuring carbonaceous matter, if necessary. The unignited residue will, needless to say, contain the pyrite of the rock and any other sulphides not attacked by dilute acid. In the case of some nonsiliceous rocks it may even consist to a considerable extent of iron oxide, probably in most cases one of the hydrated oxides, but perhaps often hematite. The presence of such a constituent in a limestone is occasionally indicated by a reddish cast of color in the fresh rock, provided there is not enough carbonaceous matter to obscure it. The existence of a ferric oxide is not always incompatible with the simultaneous presence of this carbonaceous matter.

A purple color may be due, as in some slates, to admixture of a ferric oxide or hydroxide with carbonaceous matter, while to the latter component are due the gray and black colors often encountered. The color of greenish sedimentary limestones may be caused by certain minerals of the mica family, as the chlorites. 
The condition of phosphorus when it occurs in only minute amounts is not determinable with certainty. It may sometimes doubtless be found in traces in the form of heavy phosphates like monazite and xenotime in the insoluble residues, and at others it is held by iron and aluminum, but in the highly phosphatic rocks its usual form is overwhelmingly that of phosphorite or apatite, which may, in fact, become the predominating constituent.

It is known from my analyses that vanadium is a regular though very minor constituent of limestones as well as of sandstones and igneous rocks generally. Recent tests on 100-gram lots of a great limestone formation in northwestern Mexico show that it at least carries molybdenum as well as vanadium, and also nickel. Nothing is known with certainty regarding the mode of combination of these elements in limestones, though it is quite possible, as to the vanadium at least, that it was an original ingredient of the aluminous silicates of the clayey matter, and that nickel, zinc, and lead when present are mainly in the form of carbonate or sulphide.

Carbonaceous matter has been frequently alluded to as a common constituent of secondary importance. It is absent from the white marbles and some colored decorative stones, but forms an appreciable percentage of other limestones in extreme cases.

Some kinds of bituminous limestone are characterized by a fetid odor when struck or abraded. This is perhaps due in most cases to hydrogen sulphide, either original or resulting from decomposition of organic matter. The presence of hydrogen sulphide is sometimes unmistakable, and Frof. B. J. Harrington determined its amount at about 0.02 per cent in the form of liquid inclusions in a fetid calcite from Canada.

The above remarks do not exhaust the subject of the mineral composition of limestone rocks, this term being used here and elsewhere in its generic sense. to include the magnesian varieties. They may serve as a sufficient guide, however, to those undertaking their analysis. Ignorance of the mineral composition in respect to the minor components has been a prolific source of error on the part of those analyzing these rocks.

\section{BEHAVIOR TOWARD DIFFERENT REAGENTS AS A MEANS OF DISTIN- GUISHING DIFFERENT CARBONATES.}

Although for the analyst provided with laboratory facilities it is usually of little importance to possess other means for identifying qualitatively the different varieties of carbonate rocks than the conventional methods of qualitative analysis, some of the rapid methods for so doing may serve a most useful purpose at times, especially in the field, where transport of bulky apparatus and quantities of liquid reagents is difficult or impossible.

20091-Bull. 422-10-14 
a. DIFFERING SOLUBILITY IN TARTARIC AND CITRIC ACIDS AND IN ACID POTASSIUM SULPHATE.

This subject, touched on now and then by earlier writers, was first studied by H. C. Bolton, ${ }^{a}$ and many of his results were long afterwards confirmed by J. W. Richards and N. S. Powell. ${ }^{b}$ Solid organic acids like citric and tartaric were found to be well adapted for distinguishing between many carbonate minerals by the difference in the degree of action on them. Potassium acid sulphate had earlier been used to some extent by E. Jannettaz, ${ }^{c}$ and Richards and Powell give data as to this reagent for carbonates. It will suffice here to refer to the originals, especially Bolton's papers, where will be found data as to the solubility of a great variety of minerals in the abovenamed solvents as well as in others of inorganic nature.

For the present purpose it is more important to present in brief the methods proposed for distinguishing between calcite and aragonite and between relatively pure limestones and those high in magnesium.

\section{b. THE MEIGEN REACTION FOR DISTINGUISHING CALCITE FROM ARAGONITE.}

According to W. Meigen, ${ }^{d}$ calcite powder when boiled with a dilute solution of cobalt nitrate remains white or, in presence of organic matter, becomes yellowish, while aragonite turns lilac red from formation of a basic cobalt carbonate. G. Panebianco ${ }^{e}$ obtained different results. Calcite after a minute's boiling with the cobalt nitrate reagent became sky blue and after four minutes lavender blue. Aragonite became at once lilac and later violet, the difference in the colors being supposedly due to different basic carbonates. Panebianco also observed that the reaction was useless for proving absence of calcite when mixed with aragonite, for the color characteristic of the latter still appeared when 19 parts of calcite were mixed with but 1 of aragonite. Freshly precipitated gelatinous calcium carbonate, as well as that from warm solutions, gives the aragonite reaction, but that formed at $0^{\circ}$ affords the calcite reaction.

A. Hutchinson ${ }^{f}$ seems to have shown that the disagreement between the results of these investigators is to be attributed to the presence of iron in the cobalt nitrate used by Meigen.

According to Meigen, barium and strontium carbonates, but not that of magnesium, behave like aragonite, and calcium phosphate produces a blue powder.

a Ann. New York Acad. Sci., vol. 1, 1877-1880, pp. 1, 153; vol. 2, p. 1; Proc. Am. Assoc. Adv. Sci., vol. 31, 1883, p. 271; Chem. News, vols. 36, 37, 38, 43, 47, 1877-1883; Rept. Brit. Assoc. Adv. Sci,, 1880, p. 506; Min. Mag., vol. 4, 1880-81, p. 181; Ber. Deutsch. chem. Gesell., vol. 12, 1880, p. 726.

$b$ Jour. Am. Chem. Soc., vol. 22, 1900, p. 117.

c Compt. Rend., vol. 77, 1873, p. 838; vol. 78, 1874, p. 852.

$d$ Centralbl. Min., 1901, p. 577.

e Rivista min. crist. ital., vol. 28,1902 , p. 5.

finin. Mag., vol. 13, 1903, p. xxviii. 
c. DISTINGGUSHING BETWEEN CALCITE AND DOLOMITE.

It must be understood that magnesian and dolomitic limestones represent, as a rule, mixtures of the double carbonate dolomite with calcite, and not of calcite with magnesite. Calcite is easily soluble in dilute hydrochloric and acetic acids; dolomite and magnesite are nearly insoluble. Hence calcite effervesces strongly with these acids, even in lump form, while dolomite shows little or no effervescence. An easy means is thus afforded for distinguishing the two. Dolomitic limestones are also but feebly attacked, according to their content in calcite, so that in powder form it is not difficult to make a fair separation of calcite from dolomite and magnesite, but not from magnesian hydrocarbonates, which last, however, are never essential components of the great carbonate formations.

$J$. Lemberg ${ }^{a}$ found that calcite responds by the formation of a violet color in its powder when treated with a solution of aluminum chloride and hematoxylin (extract of logwood), but that dolomite remains unaffected. The solution is prepared by dissolving 4 parts of dry aluminum chloride in 60 parts of water, adding 6 parts of logwood, and boiling for twenty-five minutes with replacement of the escaping water. The deep-violet solution is then filtered.

Coarsely powdered Iceland spar and Carrara marble became violet after five to ten minutes immersion and subsequent removal of the adhering solution with water. In the powder of a dolomitic limestone the calcite grains were easily distinguishable from those of dolomite by this means. It is not advisable to prolong the treatment unnecessarily, since after twenty minutes the dolomite surfaces may show occasional pale-blue spots. The reaction is caused by precipitation upon the calcite of aluminum hydroxide, which forms, with the coloring matter, a lake.

F. Hinden ${ }^{b}$ finds that limestone (calcite) at room temperature gives carbon dioxide with 10 per cent solution of ferric chloride, but dolomite does so only on warming. A second reagent mentioned by him is copper sulphate solution, from which basic copper carbonate is separated by calcite, but is not by dolomite.

The behavior toward heat alone is sometimes useful as a distinguishing test, for dolomite loses its carbon dioxide so easily that the powder is violently projected from the crucible if the heat is not very cautiously applied, whereas calcite can be strongly heated at the start without danger of loss of solid matter. 


\section{PART II.-REFINED METHODS OF ANALYSIS.}

32. SILICA, ITS SEPARATION FROM ALUMINA, ETC.

\section{A. METHODS OF DECOMPOSING THE ROCK.}

Few limestones are so pure that they leave no residue on treatment with hydrochloric acid. The residue may consist of quartz, clay, or other silicates, carbonaceous matter, pyrite, etc., and the mode of attack employed will depend on the amount of insoluble matter, and whether or not it is desired to know its composition separately from that of the soluble portion.

a. WHEN THE INORGANIC RESIDUE IS TO BE SEPARATELY ANALYZED.

A gram of the rock powder is moistened with water and dissolved in dilute hydrochloric acid (or acetic, if the former acid is likely to act appreciably on the silicates) in a covered beaker till all effervescence ceases. Moderate heat is needed if the effervescence is so weak as to indicate a rock of dolomitic character. The solution is filtered through a $7 \mathrm{~cm}$. filter and the residue washed with water, or hot dilute hydrochloric acid should gypsum be present and the silicates resistant. . The paper, with its contents, is ignited moist in platinum (blast needed only if the amount is considerable), and after weighing it is fused with sodium carbonate and analyzed like a silicate rock (d, p. 87 et seq.). If it contains hydrous minerals the original water content of the residue is best determined by a separate test on a fresh portion of the sample, and not by drying and weighing on a counterpoised filter and then igniting. The weight of water found should be added to that of the ignited residue in order to get the true weight of the insoluble matter.

If the ignited residue is wholly quartz, or is very insignificant in amount, it may be treated at once with a drop of sulphuric acid and a few drops of hydrofluoric acid, and the acids removed in the radiator shown in fig. 2 (p. 31). If then a slight residue still is visible, it is well to repeat the treatment with acids and evaporation, for it can not be too insistently pointed out that quartz resists the action of hydrofluoric acid far more than many silicates, and several evaporations may be needed to volatilize the crystallized mineral unless it has first been reduced to a most impalpable state of division. When the weight after exposure for a few minutes to the full burner heat no longer changes, the loss represents silica. If a slight residue still shows (it is most likely to be mainly alumina), the subsequently obtained precipitate of alumina, etc., is added to it and ignited in the same crucible. The filtrate is best treated as in 33. A. a, page 216 .

If the original residue is small, and accurate knowledge of its composition is desired, several grams of the rock may be dissolved and the 
whole filtrate best treated for dissolved iron, aluminum, and manganese as in 33. A. a, page 216, but only an aliquot part of the subsequent filtrate should then be used for the determination of calcium and magnesium.

In case the filtrate from the residue is perceptibly colored by dissolved organic matter, a condition that may occasionally arise, this organic matter must be effectually destroyed before proceeding to the precipitation of iron and aluminum, for otherwise incomplete precipitation of one or both will be the result. The complete removal of this disturbing material can not always be effected by ordinary oxidizing agents, but only by evaporation and heating to its carbonization point. In doing this it is necessary, in order to avoid possible loss of iron as chloride, to evaporate with nitric acid to dryness a couple of times and then to heat over a free flame gently till the desired result is achieved. If this seems undesirable for any reason, it may be better to start with a fresh portion of the limestone and to treat it by one of the methods under $b$, below, for the bulk analysis.

b. WHEN THE INORGANIC RESIDUE IS NOT TO BE SEPARATELY ANALYZED.

This will be the usual case. As said above, the residue may consist of clay or other silicates, carbonaceous matter, pyrite, etc., with or without quartz. There are two ways open to render the noncombustible part of this soluble.

$\alpha$. By solution in acid after strong ignition.-This is the best method to employ in all cases where the ratio of insoluble to soluble compounds is not less than is found in an argillaceous limestone that is directly suited for burning to Portland cement-that is to say, when the silica does not much exceed 15 per cent and the oxides of iron aluminum, and titanium together are not in excess of 6 per cent. The exact allowable limits have not yet been determined, nor is it known what the proportions may be in dolomites and dolomitic limestones. This is a subject for further investigation.

Limestones, however, in which the above percentages of silica, alumina, etc., are not exceeded may be converted in ten to fifteen minutes by a good blast capable of giving an effective temperature of $1,100^{\circ}$ to $1,200^{\circ}$ to a product that is wholly soluble in hydrochloric acid, provided the rock was first reduced to a very fine powder.

A gram of the powder is heated in a covered platinum crucible by an inclined blast. If a limestone, the flame may be applied at once, as a rule, without fear of loss, or after short exposure to a full Bunsen flame. Highly magnesian limestones, if this method is applicable to them, must, however, be heated with the greatest caution, for their temperature of decomposition is far below that of limestones, and violent projection of material often begins far short of visible redness. 
The strong heating is usually stopped after ten or fifteen minutes, when the shrunken product may have the appearance of a sintered or even clinkered mass that detaches for the most part readily from the crucible. $^{a}$ It is transferred to a beaker or evaporating dish and moistened with water. The crucible is then cleaned with hydrochloric acid $(1: 1)$ and the contents are poured into the beaker or dish. By gentle heat and cautious pressure with the flattened tip of a rod the lump or lumps are caused to disintegrate and pass largely into solution in a few minutes. A certain amount of silica may remain undissolved in a flocculent state, but this is of no moment. When all grit has disappeared, the liquid, if in a beaker, is washed into a dish and evaporated to dryness. If solution was made directly in the dish the volume of liquid need not exceed a few cubic centimeters and the evaporation takes but a short time. A much smaller dish is allowable than in the case of wholly siliceous minerals, because of the small bulk of solution and the entire absence of added.fixed salts.

$\beta$. By solution in acid after heating with sodium carbonate.-When the siliceous components are in such amount as not to permit the formation of a wholly soluble product by strong ignition, it is necessary to mix sodium carbonate with the power and decompose the silicates by its aid over the blast lamp. This procedure may be adopted with all siliceous limestones if desired. The amount of flux to be used is but a fraction of that needed for a silicate analysis. One-fourth to one-half gram for 1 gram of limestone suffices, ${ }^{b}$ for the lime formed by ignition is itself a powerful flux and it is quite unnecessary to have here a liquid fusion. A sintering suffices. This proportion holds also for highly magnesian limestones and for cement rocks containing as little as 50 per cent of lime. ${ }^{c}$

\section{B. SEPARATION OF SILICA.}

In this connection the remarks under $\beta$, page 92 , should be carefully read.

Because of the absence of large amounts of alkali salt and of much silica the evaporation of the hydrochloric acid solution obtained in A. a and b. $\alpha$ and $\beta$, takes little time as compared with a silicate analysis. When dry, or nearly so, on the steam bath, the dish may be placed in an air bath or (covered) on a platinum triangle resting on a hot plate, and heated to $200^{\circ}$ for an hour if a limestone is under

\footnotetext{
$a$ The changes that take place during the ignition comprise loss of all carbon dioxide, water, and carbonaceous matter; oxidation of all pyrite, with retention of the whole of the sulphur as calcium sulphate. Prolonged heating will gradually expel all the sulphur trioxide from the calcium sulphate and later the alkalies, which can be wholly volatilized in an hour or fess by a powerful blast. With an inclined blast the alkalies condense in part on the under side of the lid as a soluble and powerfully alkaline deposit, sometimes weighing several milligrams, but in the time above set no loss of alkali appears to occur.

$b$ Shimer, P. W., in Meade, R. K., The Chemist's Pocket Manual, p. 162.

$c$ Personal communication from R. K. Meade.
} 
treatment, but for a highly magnesian limestone the temperature should not exceed $120^{\circ}$, according to J. P. Gilbert, ${ }^{a}$ because of recombination of silica and magnesia, with subsequent re-solution of the silica when acid is added.

Bertram Blount claims ${ }^{b}$ that by heating to 200 in this manner the silica is completely separated by a single treatment, but I have not found it so, though the amounts going into solution on subsequent addition of acid are small, seldom over 2 or 3 milligrams. Therefore, for exact work with silica present to the amount of 2 to 4 per cent and over, that already rendered insoluble should be filtered off and the solution again evaporated. With this end in view the dry mass is drenched with strong hydrochloric acid and allowed to stand for a few minutes, then as much water is added and the dish covered and placed on the bath for ten minutes. Instead of strong acid that of half strength may be used and the heating begun at once. The silica is then separated by filtration on a filter of suitable size, washed thoroughly with dilute acid and then twice with cold water. The filtrate is evaporated again to dryness, the residue extracted with hydrochloric acid as before, but with allowance of only a few minutes' time, and the solution filtered once more through a second and smaller paper. The two papers with their contents are slowly dried, charred, and ignited in platinum, finally over the blast for ten minutes. The weighed silica is to be corrected for foreign matter by evaporating with $5 \mathrm{~cm}^{3}$ of hydrofluoric acid and one or two drops of sulphuric acid, as directed in c (p. 95). The residue obtained after ignition is almost always considerably less than that found in analyzing a silicate rock and an ignition of a minute or two over a full burner, after expulsion of the sulphuric acid, is in almost all cases sufficiont. The composition of this residue is similar qualitatively to that derived from silicate rocks. It should never contain calcium or magnesium, but consists mainly of alumina, with a little ferric, titanic, and phosphoric oxides. It is preserved and the subsequently obtained precipitate of these oxides is added to it and ignited in the same crucible.

33. ALUMINUM, TOTAL IRON, TITANIUM (SILICA, MANGANESE); PRECIPITATION IN COMPANY WITH PHOSPHORUS, ETC.c.

The next steps differ widely according to the relative amounts of iron, aluminum, etc., taken collectively, and according as it is the intention to precipitate manganese with these or after separation from them. The individual preferences of experienced analysts will often differ as to the selection of the particular method, and it is not intended here to prescribe definitely the course that anyone should follow. Nor in the following are all methods of separation that may

a Technology Quart., vol. 3, 1890, p. 61; abstract in Zeitschr. anal. Chemie, vol. 29, 1890, p. 688 .

b Jour. Soc. Chem. Ind., vol. 21, 1902, p. 1217.

c See also 8, p. 98 . 
be capable of affording good results noticed. There are some that seem promising but that have not yet been studied sufficiently in this laboratory, nor in fact elsewhere, to justify description. A method that may be well adapted to a certain combination of elements may be valueless if to that combination a single other element is added; or it may be that the possible disturbing influence of that element has not been as yet ascertained.

\section{A. PRECIPITATION OF ALUMINUM, IRON, ETC.}

a. Collective precipitation of aluminum, iron, manganese, titanium, and PhosPHORUS BY AMMONIUM SULPHIDE.

If these elements collectively represent 2 or 3 per cent or less of the rock, the filtrate from the silica (32. B, p. 214), reduced in bulk if need be, is put into a flask of $150 \mathrm{~cm} .^{3}$ capacity with enough hydrochloric acid to prevent precipitation of magnesium when made ammoniacal. This last is a most important point. Ammonia free from carbonate is then added to strong alkalinity and hydrogen sulphide is introduced to saturation, followed by a fresh addition of ammonia equal to the excess first used. Boiled water is then poured in till well up in the neck, the cork is inserted. and the flask is set aside for twelve to twenty-four hours. Its contents are then collected on a filter of small size $(7 \mathrm{~cm}$.) and washed with water containing a little ammonium sulphide and nitrate. Precipitation is not absolute as to one or more of the constituents of the separated mixture, but the amounts recoverable from the filtrate are usually very minute. (For treatment of the filtrate see 36, p. 221.)

The paper and its contents, having been washed with ammonium nitrate, may be safely charred and ignited as they are in the crucible containing the residue left after volatilizing the silica. The final weight after ignition in full contact with air, followed by application of the inclined blast for not over five minutes, represents $\mathrm{Al}_{2} \mathrm{O}_{3}$, $\mathrm{Fe}_{2} \mathrm{O}_{3}, \mathrm{Mn}_{3} \mathrm{O}_{4}, \mathrm{TiO}_{2}$, and $\mathrm{P}_{2} \mathrm{O}_{5}$. (For the separation of these, see $\mathrm{C}$, p. 218.)

This method is inapplicable for phosphatic limestones containing insufficient aluminum to more than combine with the phosphorus, for then calcium phosphate would be precipitated also. In such case method $\mathrm{b}$ or $\mathrm{c}$ would apply if the iron and aluminum together more than sufficed to carry down the phosphorus, but in general it would be necessary to add a known amount of pure iron oxidized to the ferric state, then to precipitate by ammonia or the basic-acetate process, and to deduct the added iron oxide from the weight obtained.

b. precipitation of aluminum, iron, titanium, and phosphorus by ammonia.

The filtrate from silica is boiled in a beaker of good glass, with addition of a few drops of bromine water or two or three of strong nitric acid, till all trace of bromine or chlorine is gone. Then hydro- 
chloric acid is added, if not already present, in amount sufficient to prevent precipitation of magnesium when rendered ammoniacal. Arnmonia is added to the boiling solution (in platinum if possible), 1.00 to $200 \mathrm{~cm}^{3}$ in volume, in slight excess, and the boiling is contirued for two or three minutes. The precipitate is allowed to settle in the covered vessel, collected quickly on a filter, washed with hot water two or three times, and sucked dry. It is then redissolved in hct and moderately strong hydrochloric acid, the solution is boiled to drive out the trace of chlorine derived from solution of any precipitated manganese dioxide, and the precipitation is repeated just as described once, twice, or even three times, according to the amount of manganese in the rock. Macerated filter paper is added before the last precipitation if the oxides are in large amount. The final precipitate is washed with water containing a little ammonium nitrate. (Fior treatment of the filtrate, see B, below.)

The precipitate is ignited moist in the crucible containing the residue from volatilization of the silica by hydrofluoric acid, and the combined weights increased by traces subsequently recovered ( $\mathrm{B}$, below) represent all iron as $\mathrm{Fe}_{2} \mathrm{O}_{3}, \mathrm{Al}_{2} \mathrm{O}_{3}, \mathrm{TiO}_{2}$, and $\mathrm{P}_{2} \mathrm{O}_{5}$. (For separation of the ignited oxides, see C, p. 218.)

c. precipitation of aluminum, iron, titaniUm, aNd phosphorus by the basicACETATE METHOD.

This method of separation is less employed in limestone analysis than in that of silicate rocks, by reason of the relatively smaller amounts of those elements that precipitate in the basic-acetate process, and hence of their more ready separation by ammonia from appreciable quantities of manganese. (For its application, see A. b. $\beta$; p. 100 , giving due regard to the smaller amounts of iron, etc., involved in the case of limestones.)

\section{B. TREATMENT OF THE FILTRATES FROM ALUMINA, ETC.}

The combined filtrates obtained in A. b (or c), above, which, besides all the calcium and magnesium, will contain the manganese and usually traces of aluminum and even iron, are evaporated to a small bulk in platinum with the addition of a few drops of ammonia from time to time, and any precipitate that separates is collected. This is washed a little, redissolved in hydrochloric acid (with addition of a drop or two of sulphurous acid if its color indicates precipitation of some manganese), and reprecipitated by ammonia at boiling heat in a very small beaker. If need be the precipitation is to be again repeated, but this will seldom be the case. A first precipitation is, however, called for in order to separate a little calcium carbonate that may have precipitated during the evaporation of the original ammo- 
niacal filtrate. The alumina and perhaps trace of iron oxide thus obtained are added to that already found (A. b or c, pp. 216-217). (For treatment of the filtrate, see 34, p. 219.)

\section{SOLUTION AND SEPARATION OF THE OXIDES OBTAINED IN A AND B.}

The same course is followed, whether method a, b, or c under A, pages 216-217, has been employed for precipitating aluminum, iron, etc. According to the weight of the ignited oxides, these are fused with from one to several grams of potassium or preferably sodium pyrosulphate ${ }^{a}$ in the covered crucible over a low flame. By using pyrosulphate instead of acid sulphate the time of fusion is greatly shortened, especially when the oxides are in a finely divided state, as after precipitation in presence of macerated filter paper.

When decomposition is complete the cooled melt is dissolved in hot dilute sulphuric acid, the solution is transferred to a capacious platinum dish and evaporated as far as practicable on the bath. Strong sulphuric acid is cautiously added, and evaporation is continued over a radiator, or on a sand bath, or by aid of a free flame applied with care to prevent spattering, till fumes of acid appear freely. When cool, the melt should be rather pasty and not hard and dry. It is dissolved by the aid of heat in a moderate amount of water, and the solution is allowed to digest for ten or fifteen minutes on the bath. A few flocks of silica will then generally be apparent at the bottom of the dish. Whether visible or not, the solution is passed through a $7-\mathrm{cm}$. filter, which is then washed with hot water till free from salts.

$$
\text { a. SILICA (BARIUM). }
$$

The paper with the silica is ignited, weighed, and then treated.with a drop of sulphuric acid and a few drops of hydrofluoric acid and the crucible, after ignition, is again weighed. The loss represents silica that escaped earlier separation, and its amount is to be added to that already found.

If a final residue is reddish, it is to be brought into solution by a little pyrosulphate and its solution, if clear, added to the main filtrate containing the mass of the iron. If not clear, the turbidity may be due to a trace of barium sulphate, which should of course be separated and deducted from the weight of the alumina plus other oxides.

$$
\text { b. IRON. }
$$

In this solution the iron is to be determined by potassium permanganate after reduction by hydrogen sulphide, exactly as detailed under 8. B.a, page 107. The amount of vanadium present is, so far as known, always so small as to make it unnecessary to follow the procedure under 8. B. b, page 109. 
c. TITANIUM.

After titration of the iron the solution is treated exactly as prescribed under 8 . B. a. $r$, page 109 , and the titanium is determined colorimetrically (p. 128).

\section{d. ALUMinum.}

The aluminum is determined by difference after subtracting the weights of iron and titanium oxides found as above, and also the phosphoric oxide found in a separate portion of the sample (p. 222), and further the manganoso-manganic oxide, if this had intentionally been precipitated along with the iron and aluminum as in $A$. a, page 216. (For the discussion of the relative merits of this indirect and the direct methods for arriving at the aluminum, see 8. A. a, p. 98, and C, p. 111.)

\section{MANGANESE.}

A. IN THE FILTRATES FROM 33. B (p. 217).

The filtrates containing the calcium, magnesium, and manganese are caught in a flask of about $150 \mathrm{~cm}^{3}$ capacity, $2 \mathrm{~cm} .^{3}$ of ammonia are added, and hydrogen-sulphide gas is introduced to saturation, then additional ammonia is added to equal that just prescribed.' The flask is set aside, stoppered for twelve to twenty-four hours, the precipitate (perhaps colored dark by a little platinum, iron, or other heavy-metal sulphide ${ }^{a}$ ) is collected on a $7-\mathrm{cm}$. filter and washed with a dilute solution of ammonium chloride carrying a little ammonium sulphide. $^{b} \quad$ (For treatment of the filtrate, see 35, p. 220.)

If the manganese is not to be determined in a separate portion of the rock, the flask is then cleaned from possibly adhering sulphide with a solution of half-strength hydrochloric acid diluted with five times its volume of hydrogen sulphide water, which is then passed through the filter in order to dissolve the manganese sulphide. The fiitrate is treated exactly as under 9 . B. b, page 115, if the gravimetric method of determination is chosen. In view of the relatively enormous errors affecting this method when small amounts are in question (see 9. A, p. 113), it is far safer to evaporate the hydrochloric acid solution with nitric or sulphuric acid, till no trace of chlorine is left, and to apply the colorimetric method (9. C. b, p. 117).

If the manganese is to be determined in a separate portion, the sulphide precipitate is discarded; indeed, if it is known that but a few

a More than a negligible trace of iron should not appear at this point. If, however, the organic matter of the limestone is at all soluble in acids and the method of direct solution has been employed, it may very well happen that some iron (aluminum likewise) will fail of precipitation by ammonia. The former will thın appear at this stage. Fence, prior ignition of the limestone is imperative in such cases.

1. This is best prepared by considerably overneutralizing a little hydrochloric acid in a small beaker, and then adding hydrogen sulphide water or introducing hydrogen sulphide gas for a few moments, auo. further diluting if need be. 
hundredths of a per cent are present, its separation at this stage may be altogether omitted.

\section{B. SEPARATE DETERMINATION OF MANGANESE.}

Small amounts are always best determined by colorimetry, and then with greatest accuracy in a separate portion of the sample. This, if wholly soluble in acid, is to be dissolved by dilute nitric acid free from hydrochloric acid, the solution filtered if necessary, and further treated, in whole or in an aliquot part, as in 9. C. b, page 117. If not altogether soluble in acid or if the solution is colored by organic matter, it is best to heat the powder with half its weight of sodium carbonate (itself free from manganese) over a strong blast, to digest the cooled mass with nitric acid till any residue that may remain is entirely colorless, to filter into a suitable-sized flask, and to determine as in 9 . C. b, page 117 .

35. COPPER, NICKEL, COBALT, LEAD, ZINC, RARE EARTHS, CHROMIUM, VANADIUM, MOLYBDENUM.

It is useless, as a rule, to look for any one of these constituents in the amount of sample taken for the general analysis. From 50 to 500 grams of material will be required. This is dissolved in hydrochloric acid and the residue separated by filtration. If it is desired to examine this residue it is best done by analyzing it separately, after fusion with sodium carbonate and leaching with water. Vanadium and molybdenum will then be found in the filtrate, the other metals in the residue. The former can be determined according to 18, page 148; the latter by the usual methods.

For the hydrochloric acid solution; which will contain the above elements mainly or wholly, the treatment given under 33 . A. a, page 216 , is probably best adapted, and the following outline may serve as a guide:

The precipitate produced by ammonium sulphide is to be dissolved, the copper and perhaps some molybdenum precipitated by hydrogen sulphide, the filtrate oxidized and evaporated to dryness, and the residue boiled with a solution of oxalic acid. The rare earths, perhaps contaminated by silica, will be thrown out and are to be washed with dilute oxalic acid solution, ignited, treated with sulphuric and hydrofluoric acids, redissolved and reprecipitated in neutral or nearly neutral solution by oxalic acid, ignited, and weighed. They can then be tested as to their character by methods to be found in special treatises on the rare earths. The filtrates are to be freed from oxalic acid by evaporation and gentle ignition and the residue redissolved and tested for nickel, cobalt, zinc, chromium, vanadium, and molybdenum by approved methods. It must not be forgotten 
that the filtrate from the original precipitaticn by ammonium sulphide may hold vanadium, molybdenum, and perhaps nickel, which ere to be recovered by acidifying with dilute acetic acid.

Vanadium may usually be found and determined with sufficient Eccuracy in 5 grams of the limestone by fusing with sodium carbonate snd further treating as in 18. C, page 150 .

\section{CALCIUM, STRONTIUM, BARIUM, MAGNESIUM (MANGANESE).}

The filtrate obtained in 33. A. a, page 216 , or 34 . A, page 219 , is ireated for the most part as in silicate analysis (pp. 118-128).

With dolomitic limestones and dolomites it will be usually impossible to obtain a correction by the method given in C. b, $\beta$, page 127 , for the small amount of calcium with the magnesium pyrophosphate. 'The first of the methods there given $(\alpha)$ is to be followed.

The accurate determination of barium in a limestone is more difficult than in a silicate rock. It is perhaps best to recover it, if present, from the filtrates from the calcium oxalate, after evaporating them and expelling ammonium salts, by taking up the residue in as little hydrochloric acid as possible and, without filtering, adding a few drops of sulphuric acid and allowing to stand for several hours. The washed and ignited precipitate is evaporated with a drop of sulphuric acid and a few drops of hydrofluoric acid to remove silica, the final residue is dissolved by a little hot strong sulphuric acid, and the resulting solution poured into a few cubic centimeters of cold water, the crucible being well rinsed with cold water by the aid of a feather or "policeman." If a precipitate now appears it is barium sulphate, which is collected and weighed in the customary manner.

For the utmost refinement in the determination of the magnesium it is probably best to evaporate the filtrates from the calcium oxalate to dryness, expel the ammonium salts by ignition, add hydrochloric acid and reevaporate, take up again with as little acid as possible, and filter through the smallest filter. The filter is preserved for later use. To the filtrate, which should be very small in volume, a few drops of sulphuric acid are added, and if after some hours a precipitate of barium sulphate has appeared it is collected and burned in platinum with the previous filter and its contents. The ignited matter is treated as in the preceding paragraph.

To the filtrate is added enough hydrochloric acid to prevent precipitation of magnesium when rendered ammoniacal. Ammonia in slight excess is added and the solution is boiled for some minutes in a platinum dish. There will probably appear a very slight precipitate containing aluminumhydroxide, which is to be redissolved and reprecipitated. In the filtrate the magnesium is precipitated, together with any manganese that may be present, in the manner elsewhere detailed (p. 124). 


\section{PHOSPHORUS.}

The rock is dissolved in dilute nitric acid, the solution filtered, the residue fused with a little sodium carbonate, and its nitric acid solution added to the other.

Or, if the rock is rather argillaceous, the powder may be mixed with half its weight of sodium carbonate and strongly blasted. The nitric acid solution of the mass is then evaporated to dryness to remove most of the silica, which is collected on a filter, washed, ignited, and evaporated with hydrofluoric and nitric acids. The evaporation with nitric acid alone is repeated once or twice to expel the fluorine, and the residue is dissolved finally in nitric acid and its solution added to the former.

To the solution obtained in either of the above ways ammonium molybdate solution is added and the precipitate treated by one of the approved methods.

\section{FERROUS IRON.}

The presence of carbonaceous matter in limestones renders the exact or even approximate determination of ferrous iron often impossible. Nevertheless, even in its presence acceptable results are sometimes obtainable if there is not much of such matter and if it does not give with acid a colored solution. But even then it is only the soluble iron, existing chiefly if not wholly'as carbonate, that is determinable. Occasionally limestones show films of manganese peroxide, which likewise interferes with the determination.

\section{A. IN ABSENCE OF CARBONACEOUS MATTER.}

a. FERROUS IRON SOLUBLE IN SULPHURIC ACID.

The powder, one to several grams, is introduced into a stout flask of about 200 to $250 \mathrm{~cm}^{3}$. capacity and boiled with a little water till all air is expelled. While still boiling, dilute sulphuric acid is added, a little at a time, till effervescence ceases, and then a further amount. Calcium sulphate precipitates, but the iron will remain in solution. The flame is then removed and a stopper tightly inserted, through which passes a small stopcock funnel. When cool or nearly so, cold water is poured into the funnel, the cock cautiously opened, and the water drawn into the flask, more water being poured into the funnel as fast as it empties, till the solution amounts to 100 or $150 \mathrm{~cm} .{ }^{3}$ Such precaution to exclude air is hardly necessary in most cases, however, for in presence of sulphuric acid the oxidation of ferrous iron is exceedingly slow. It is ordinarily quite sufficient to equalize the internal and external pressures by opening the cock, removing the stopper, and pouring in cold water. The flask is then brought under a burette containing dilute permanganate solution and the iron titrated without 
delay. With a carbonate which is wholly decomposable without the aid of heat, solution may be accomplished in a flask filled with carbon dicxide, using cold acid, whereby the danger of attack of silicates is lesisened if these are present.' If the preference is for the potassiumbichromate method of titration, hydrochloric acid may be used instead of sulphuric, in absence of manganese peroxide. In this case there is, of course, no separation of an insoluble calcium salt, a fact which renders easier the subsequent determination of the iron in any insoluble residue the rock may yield. The ferrous iron thus found is mostly, if not altogether, that existing as carbonate.

\section{b. FERROUS IRON IN THE INSOLUBLE RESIDUE.}

The titrated solution is filtered through a medium-sized hardened filter, the insoluble matter collected thereon and washed with water. The contents of the paper are rinsed into a capacious platinum crucible, the water mostly evaporated, and in the residue the ferrous iron is detiermined as in 19. D. d, page 167.

'The total ferrous oxide found in a and $b$, when calculated to ferric oxide and subtracted from the total iron in the same state, gives the ferric oxide in the rock. If separate analyses have been made of the parts soluble and insoluble in dilute acid, similar calculations applied to the iron derived from the two portions will give the ferric oxide in each.

C. TOTAL FERROUS IRON.

Jf separate determinations are not desired, water is poured upon the powder in a capacious platinum crucible, dilute sulphuric acid is slowly added till effervescence ceases, then the crucible is placed in one of the forms of apparatus described in 19. D. d, page 167, together with $5 \mathrm{~cm}^{3}$ of hydrofluoric acid, the decomposition of the silicates is effected in an atmosphere of carbon dioxide, and the titration is made as there directed.

\section{B. IN PRESENCE OF CARBONACEOUS MATTER.}

Jecomposition is effected in a flask by dilute sulphuric acid in an atroosphere of carbon dioxide. With limestones and active agitation no heat need be used, but with dolomites it will be necessary. The solution is then quickly filtered through asbestos (in an atmosphere of carbon dioxide if much iron is present), the residue and filter washed a few times, and the filtrate titrated at once with permanganate. If it is colored by organic matter, the result may be in error.

As in A. a, page 222, hydrochloric acid and titration by dichromate may be used in absence of manganese peroxide. In either case it is important to allow the acid to act no longer than is necessary and to filter quickly, A determination of the ferrous iron in the insoluble 
matter is usually not worth attempting, because of the admixed organic matter.

\section{ALKALIES.}

The alkalies in carbonate rocks are, of course, constituents of the siliceous components. Since these are usually subordinate, it is advisable to operate on not less than 1 gram of the powder, and the J. Lawrence Smith method is to be followed in nearly all its details (p. 171). Because of the presence of a large amount of calcium carbonate in the rock it might seem that addition of precipitated carbonate would be needless. That it is not, however, appears to have been shown by experiments made with and without its addition. A little more alkali was obtained in the former case, a result that is probably due to more effective reaction of the finely divided artificial carbonate than of the crystalline natural carbonate with the ammonium chloride. One-half the usual amount of precipitated carbonate will suffice.

\section{CARBON DIOXIDE, CARBON (WATER).}

\section{A. DETERMINATION OF CARBON DIOXIDE.}

This determination is made precisely as under $21 . \mathrm{B}$, page 179 . If the absorption tubes have a height of about $10 \mathrm{~cm}$. and a diameter of $1.2 \mathrm{~cm}$. the first one will need refilling after every second analysis. For this reason Liebig or Geissler bulbs containing potash solution may be preferred.

\section{B. DETERMINATION OF CARBON OF CARBONACEOUS MATTER.}

The character of the carbonaceous matter in limestones has not been made the subject of study, so far as known to me, but it seems reasonable to assume that it resembles coaly matter rather than the indefinite humus. Although on solution of the rock in dilute acids the odor of the escaping gas is sometimes indicative of volatile organic substances, the amount thus escaping can never be more than a small fraction of the total. If the rocks are boiled with acid the filtrate may now and then be somewhat colored, but if care is taken to use dilute acid and to filter as soon as its action on the carbonates is over, little or no organic matter is likely to pass into solution. Hence, in all such cases the following method for the determination of its carbon is indicated, provided sulphides are practically absent:

Treat 1 to 10 grams of the rock with dilute hydrochloric acid till the carbonate constituents are surely decomposed, filter through an asbestos plug filling a constriction in a glass combustion tube, wash thoroughly with water, dry, and burn the carbonaceous matter over an ordinary burner in an air or oxygen current, collecting the escaping water in a calcium-chloride tube and the carbon dioxide in one filled 
with soda-lime. The combustion need occupy but a few minutes, as a rule.

If the rock is free from hydrous minerals, if the asbestos likewise yields no water on ignition, and if the asbestos and its load have been thoroughly dried, from the gain in weight of the calcium chloride tube the hydrogen of the organic matter may be calculated. These conditions will, however, seldom be fulfilled, so that only the carbon has to be considered. In fact, it is quite useless, ordinarily, to attempt to determine the organic matter as such, either directly or by calculation. One must perforce be content with knowing its contained carbon.

C. SIMULTANEOUS DETERMINATION OF WATER AND OF TOTAL CARBON IN BOTH FORMS.

In presence of sulphides or soluble organic matter the following method will sometimes answer for the simultaneous determination of the first two or even all three of the above-enumerated constituents.

Ignite in a current of air $1 \mathrm{gram}$ of the rock in a tube of very hard glass, or in the tubulated crucible of Gooch (fig. 16, p. 78), collecting the water and the carbon dioxide as in B. The sulphur of sulphides will be retained as calcium sulphate. It is necessary for the success of this operation that the temperature be sufficient to drive out all carbon dioxide from the carbonates, but insufficient to decompose calcium sulphate. The water found represents that in the minerals of the rock as well as that derived from the hydrogen of the organic matter.

A separate determination of the carbon dioxide (see A, p. 224) gives the data for calculating the carbon of the organic matter.

According to G. T. Morgan ${ }^{a}$ the two conditions of carbon can be successively determined on the same portion of sample by first finding the carbon dioxide as in $\mathrm{A}$, but with substitution of orthophosphoric for hydrochloric acid, and then, after weighing and replacing the absorption tubes, adding chromic acid and renewing the boiling to oxidize the organic matter.

41. CHLORINE.

Several grams of the rock are dissolved in the cold, or with as low a heat as may be, in dilute nitric acid free from chlorine, the solution is filtered, silver nitrate added, and the precipitate collected and treated as usual. (See 22. A, B, and C, pp. 182-184.)

If the rock is likely to contain chlorine-bearing silicates, it is decomposed with its own weight or less of chlorine-free sodium carbonate in a platinum crucible over the burner or moderate blast without undue prolongation of the heating. The mass is extracted with hot

a Jour. Chem. Soc., vol. 85, 1904, p. 1001.

20091-Bull. $422-10-15$ 
water and the filtrate acidified with cold dilute nitric acid, or it may be decomposed directly with the acid and the solution filtered. The further treatment is as above given.

\section{FLUORINE.}

As with silicate rocks, an exact determination of fluorine in carbonate rocks is difficult if not impossible with our present methods, and little or nothing has been done along this line.

Because of the great preponderance of lime, direct fusion with sodium potassium carbonate and silica is of somewhat doubtful practicability. Probably the following treatment will afford the best results:

Dissolve the powder in dilute acetic acid, as far as this may be possible without boiling, and filter. To the filtrate add solution of sodium carbonate till in some slight excess, boil, and treat the precipitate of calcium carbonate and possible fluoride as on page 186. Most of the fluorine that may have been dissolved by the acetic acid will thus be found. The siliceous matter insoluble in acetic acid is fused with sodium-potassium carbonate and further treated as in 23. B. a, page 184 .

\section{SULPHUR.}

\section{A. TESTS AS TO ITS CONDITION; DETERMINATION OF SULPHATE} SULPHUR.

In very many, perhaps most, carbonate rocks any sulphur present is chiefly if not wholly in the sulphide condition, and then usually as pyrite. In what condition it may be is readily ascertainable. If moistened lead-acetate paper is blackened when held over the mouth of a test tube in which some of the rock powder is being boiled with dilute hydrochloric acid, a soluble sulphide is indicated, but it is seldom more than a negligible trace. If the filtrate after approximate neutralization with ammonia gives a precipitate with barium chloride there is sulphate present. If the total sulphur obtained as in $\mathrm{B}$, below, is in excess of that in the sulphate and soluble-sulphide states, the difference may safely be calculated to iron disulphide.

In the presence of much sulphide and also of sulphate sulphur the extraction of the latter should be made in an atmosphere of carbon dioxide, but usually this precaution is quite unnecessary.

\section{B. DETERMINATION OF TOTAL SULPHUR.}

a. METHODS OF CONVERTING SULPHIDES TO SULPHATES.

$\alpha$. By ignition without flux.-In a rock that is not heavily charged with sulphides the following method of my own has afforded excellent results, even in the presence of 1 per cent of organic matter, though its range of application has not been ascertained: 
The platinum crucible containing 1 or 2 grams of the rock powder is placed in the perforated disk (fig. 3, p. 32) and exposed to the heat of a burner for fifteen minutes or to that of an inclined and moderate blast for ten minutes. So far as present experience teaches, it is not at all necessary to begin with a gentle heat. All sulphide sulphur is oxidized and retained by the lime as sulphate without any loss by volatilization, all organic matter is removed, and the silicates are rendered soluble in acid if they are not in excess. (See 32. A. b. $\alpha$, p. 213.)

$\beta$. By ignition with sodium carbonate.-One to 2 grams of powder are mixed with half the weight of sulphur-free sodium carbonate in a platinum crucible and heated in the perforated disk, as in $\alpha$. It is not probable that the addition of niter is called for, even when a good deal of carbonaceous matter is present. Since not enough flux is used to produce more than a sintering, the air entering the crucible after the bulk of the carbon dioxide has passed off effects very speedy oxidation in the porous mass.

With highly impure limestones it may be necessary at times to increase the amount of flux and to use a little niter as well.

\section{b. TREATMENT AFTER IGNITION.}

The ignited mass obtained in a, $\alpha$ or $\beta$, will usually separate easily from the crucible. It is transferred to a small beaker and covered with water. The crucible is cleansed with dilute hydrochloric acid and the solution poured into the beaker. More acid is added till decomposition is complete in the cold or on gently warming. The solution is then filtered, diluted if need be to 150 or $200 \mathrm{~cm}^{3}$, brought to boiling or simply placed on the steam bath, barium chloride added, and the precipitated sulphate collected in due time and weighed as usual.

Evaporation to dryness to separate the dissolved silica is quite unnecessary. After decomposition with acid the solution should never be permitted to gelatinize, and it needs but little practice on the part of the operator to insure unfailing success in preventing this. If it should happen, however, it is generally best to begin over, but the difficulty may be overcome if but little free acid is present by dissolving the gelatinized silica in sulphur-free fixed alkali and reacidifying in the cold with hydrochloric acid.

44. WATER.

\section{A. HYGROSCOPIC WATER.}

From one to several grams of the air-dry powder are heated in a current of air dried by calcium chloride in a glass tube at 100 to $105^{\circ}$ and the escaping water collected in a calcium chloride tube, or the air may be dried by sulphuric acid and the water collected in sulphuric 
acid. This direct determination of the water is certain to give a higher and more correct result than that obtained by drying in a crucible at the above temperature.

\section{B. COMBINED WATER.}

This may be ordinarily determined on the air-dry sample by heating in a tube of combustion glass and collecting the water in a calcium chloride tube with the above-mentioned precautions. Or the powder may be mixed with dry sodium carbonate and heated in the apparatus of Gooch (5. C. e, p. 78) or with anhydrous borax according to Jannasch (5. C. g, p. 82). From the result thus found the separately determined hygroscopic water is to be deducted. It is to be remembered that the result will be in error by the amount of water afforded by the hydrogen of any organic matter there may be in the limestone.

\section{PART III.-CONDENSED ANALYSIS.}

For many purposes, especially those with a technical end in view, a highly refined or detailed analysis is not called for. The analyses of this kind that are now made vastly exceed in number those demanding the kind of work provided for in many of the sections of Part II. To meet the needs of those having work of this kind to do, a procedure is here given in brief that covers the important constituents of limestones. The operations are generally given without explanations. Those who seek reasons for one or another procedure or details of manipulations are referred to the corresponding sections of Part II.

\section{DECOMPOSITION AND SOLUTION.}

If the substance is convertible by blasting into a condition wholly decomposable by hydrochloric acid, one-half to 1 gram of the fine powder is strongly. ignited in a covered platinum crucible over the blast for fifteen minutes, or longer if the blast is not very powerful.

If the material is very impure, one-half to 1 gram is to be mixed with half its weight of pure sodium carbonate and ignited over the burner and blast till well sintered together.

The ignited material obtained in either of the above ways is transferred to an evaporating dish, preferably of platinum, and moistened with a few cubic centimeters of water. The crucible is cleaned with dilute hydrochloric acid, the solution poured into the dish, which is to be kept covered if sodium carbonate was used, and more acid added. Solution of the mass may be aided by gentle pressure with the flattened end of a glass rod. The solution is then evaporated to dryness on the steam bath. It will do no harm with limestones low in magnesium to hasten dehydration of the silica by placing the dish with its dry contents in an air bath at an elevated temperature or on a triangle resting on a hot plate at $200^{\circ}$ for an hour or less. 
46. SILICA.

The residue is treated with 5 to $10 \mathrm{~cm} .^{3}$ of strong hydrochloric acid, which is then diluted to half strength, or upon the residue may be poured at once a larger volume of acid of half strength. The dish is then covered and digestion allowed to go on for ten minutes on the bath, after which the solution is passed through a $7-\mathrm{cm}$. paper and the silica washed with water, cold at first and hot later, or with hot dilute hydrochloric acid at first, followed by a little hot water. The filtrate is again evaporated to dryness, this time only on the steam bath, after which the residue is digested with acid as before, but in smaller amount, and the second portion of usually dark-colored silica is collected on another small paper. The filter papers with their contents are slowly charred in a platinum crucible, then ignited, first over the burner, finally over the blast for five to fifteen minutes, according to the amount of the silica. If the blast is weak, a second application of it should follow the first weighing: The finally weighed silica is moistened with water and two drops of sulphuric acid and 5 to $10 \mathrm{~cm} .^{3}$ of pure hydrofluoric acid are added, the crucible is placed in a radiator (fig. 2, p. 31), and the liquid wholly evaporated. The residue is blasted for a minute and in due time weighed. It is better not to make this correction if it is not intended to separate and determine also the silica always accompanying the iron and aluminum oxides, but it should never be omitted if this latter correction is contemplated.

47. ALUMINUM, IRON, ETC.

The filtrate, which need not exceed $100 \mathrm{~cm} \cdot{ }^{3}$ in bulk with fairly pure limestones, is brought to boiling in a beaker, or preferably in platinum, and made alkaline with ammonia after adding a few cubic centimeters of bromine water, and if need be enough hydrochloric acid to insure a total of 10 to $15 \mathrm{~cm} .^{3}$ of strong acid. The ammonia should not be in such excess as to require long boiling to expel the most of it, nor is the expulsion of the whole of it necessary or indeed desirable. As soon as the precipitate settles the solution is passed through a filter of suitable size and the precipitate is washed a few times with hot water and sucked fairly dry. It is redissolved in hot hydrochloric acid and precipitated again by ammonia in presence of bromine, but with less ammonium chloride and in a smaller bulk of solution. If the filter is still in good condition, it is used also for this precipitate, which is washed with hot water and sucked dry at the pump. With high iron and magnesium the precipitation may well be repeated.

The re-solution of the first precipitate may be made with nitric acid if thought desirable. Its use involves very little washing of the second precipitate. To reduce the amount of aluminum passing into 
the filtrate, the precipitated oxides may be washed with water containing in the liter $20 \mathrm{~cm}^{3}$ of nitric acid that has been neutralized with ammonia.

The paper containing the final precipitate is placed in a platinum crucible, slowly charged, then ignited over a gradually increased flame, and finally over the blast for two to five minutes. The weight found represents the oxides of ferric iron, aluminum, titanium, phosphorus, and manganese, the last presumably $\mathrm{Mn}_{3} \mathrm{O}_{4}$.

The iron, titanium, and silica in the ignited oxides are determined, after fusion with potassium or sodium pyrosulphate, exactly as detailed in 33. C, p. 218. The phosphorus and manganese having been determined in different portions (37, p. 222, and 34. B, p. 220), the aluminum oxide is found by subtracting all these oxides from the original weight of the mixture. Often the percentage reported as alumina includes in reality the titanium, phophorus, and some of the manganese, where no attempt has been made to separately determine these.

\section{CALCIUM.}

The combined filtrates from the iron, aluminum, etc., are concentrated if need be to a bulk of 200 to $250 \mathrm{~cm}^{3}$, with occasional addition of a few drops of ammonia. If a slight precipitate separates, it is collected on a small filter, washed with water, redissolved in hydrochloric acid, and reprecipitated by ammonia. It is added to and treated with the main quantity of iron and aluminum oxides, and the filtrates are combined. To them is added while boiling $20 \mathrm{~cm}^{3}$ of a saturated solution of ammonium oxalate, and the boiling is continued until the precipitated calcium oxalate assumes a. well-defined granular form. It is then allowed to stand for an hour, filtered, and washed a few times with hot water. The precipitate and filter are ignited in platinum till the oxalate is converted to oxide, which is redissolved in hydrochloric acid and the solution made up to $100 \mathrm{~cm}^{3}$. Ammonia is added in slight excess to the boiling solution and also ammonium oxalate to reprecipitate the lime. After an hour the oxalate is collected, washed a few times with small amounts of hot water, ignited over the blast, and weighed as the oxide.

Instead of igniting and weighing as oxide it is permissible to dissolve the oxalate in sulphuric acid, taking care to extract the filter thoroughly with hot dilute acid, and to titrate the solution with permanganate.

\section{MAGNESIUM.}

To the combined filtrates from the calcium precipitations are added sodium ammonium phosphate solution in excess and, when the precipitate has. formed after vigorous stirring, ammonia in con- 
siderable excess. After several hours, twelve or fifteen if the precipitate is very slight, the solution is filtered, the precipitate redissolved in hydrochloric acid and reprecipitated by ammonia in moderate excess and a drop of the phosphate precipitant in the usual manner. After two hours the precipitate is filtered on paper or on a Gooch filter, ignited, and weighed as pyrophosphate.

50. ALKALIES.

See 39 , p. 224.

51. CARBON DIOXIDE.

See 40, p. 224.

52. SULPHUR.

See 43, p. 226.

53. WATER.

See 44, p. 227 .

54. IGNITION LOSS.

This determination has little significance, though it affords a more or less correct indication of the proportion of carbonates in the rock. Since it is a determination commonly made in commercial laboratories, some space will be devoted to it.

Loss on ignition with carbonate rocks represents, as with those of a silicate nature, the algebraic sum of a number of chemical changes involving both losses and gains, and its amount will depend largely on the temperature employed. At the temperature of a moderate blast, with crucible covered, carbon dioxide, water, and carbonaceous matter escape wholly. Sulphides are oxidized to sulphates and all the sulphur is retained as sulphate by the calcium. With a powerful blast the sulphate is decomposed gradually, with eventual loss of the entire sulphur. At this point the alkalies begin to escape as oxides, the potassium relatively faster than the sodium, and they may be found in part condensed on the lid of the crucible. If the heating is long continued they can be wholly volatilized. As a small offset to these losses the iron of pyrite and the iron and manganese of ferrous and manganous carbonates take up oxygen and 
unite with the lime. By proper regulation of the temperature it is not difficult to prevent loss of sulphur and alkalies, and this is the object to be sought in making the determination.

One-half to 1 gram of the powder is placed in a platinum crucible of 20 to 25 grams weight, the crucible is inserted to three-fifths of its depth in a perforated platinum disk or asbestos board (fig. 3, p. 32), and an inclined blast flame of considerable power is caused to play against the bottom of the covered crucible for fifteen or twenty minutes. A repetition of the heating for five minutes will usually cause little or no further loss. Duplicate determinations should agree within 0.2 per cent, and after the operator becomes skilled probably within 0.1 per cent. A muffle can be substituted for the blast. In my experiments the crucibles occupied a position 2 inches inside the closed door of the muffle. It is, however, important that each operator should ascertain just what the proper conditions may be for his particular blast or muffle. They are right if after the ignition he finds exactly the whole of the sulphur in the ignited powder in the sulphate state. 


\section{IN D E X.}

A. Page.

Abrasion of crusher of mortar.

Absorption tubes, use of, in water determination .................. 73-83

Acids, quality of . ................... 39

Acknowledgments to those aiding......... 15

Alkalies, determination of ............. 171-17s determination of, apparatus for, figure showing.

172

Allen, E. T., cited .................. 56, 113, 196

Allen, E. T., and Day, A. L., cited...... 48-49, 68 pycnometer method of . . . . . . . . . . . . . 48-49

Allen, E. T., and Johnston, I., cited. 194 (footnote), $195,196,197$

Aluminum, concealment of, by manganese. 114 direct determination of ............ 111-113 indirect determination of. . . 98-110, 215-219, 229 ignition of precipitate of oxide of...... 104-105 precipitation of . . . 99-103,112-113,216-217, 229 recovery of, from filtrates. . . . . 103-104, 217-218 separation of from iron............ 111-113

Aluminum chloride, loss of 99

Ammonia, occurrence of. in rocks........ 202-203

Ammonia reagent, preparation of......... 40

Ammonium chloride reagent, quality of..... 40 preparation of . . . . . . . . . . . . . . 99,171

Ammonium oxalate reagent, quality of ...... 40

Ammonium persulphate reagent, purification of ............. 102 (footnote)

Analysis, allowable error in............... 28 completeness and thoroughness of, importance of . . ............. 16-22, 42

crushing of rock for . ................ $50-52$

diversity of, for same rock............ 18-19

grinding rock for $\ldots \ldots \ldots \ldots \ldots \ldots \ldots \ldots . .52-56$

methods of.

14

neglect of certain factors in, consequences of......................... 19-21

preparation of sample for. . . . . . . . . . 50-57 quantity of rock required for.... 22, 42, 50, 56-57 statement of.

time required for $\ldots \ldots \ldots \ldots \ldots \ldots \ldots \ldots$

See also Carbonate rocks; Silicate rocks.

Analysis, qualitative, usefulness of..........

Appliances and apparatus, descriptions and figures of . ................. 30-3s

Aragonite, tests for .................. 210 Atterberg, A., cited.............. 174 (footnote)

Austin, M., and Gooch, F. A., cited. 116 (footnote), $123-124,145$

Autenrieth, W., cited
B.

Page.

Bailey, G. F., cited................ 141, 142

Barium, determination of . 119-121, 138-140, 218, 221 ignited silica free from $\ldots \ldots \ldots \ldots \ldots \ldots . \quad 97$ precipitability of, by ammonium persulphate................... 102 occurrence of, in rocks........... 20,25, 198 recovery of, from alumina precipitate... 107 removal of, from magnesium pyrophosphate.................... 126 separation of, from strontium........ 119-120 Barium sulphate, spectroscopic testing of.... 140 Barus, Carl, cited......................... 61 Baskerville, C., method of, in determination of titanium................ 137

method of, in determination of zirconium..................... 142

Baubigny, $\mathrm{H}$., method of, in determination of chromium .............. 146

Bayly, P. G. W., cited ................ 71

Bemmelen, J. M. van, cited. ..........57,62

Berger, C.W., and Fulett, G. A., cited. 94 (footnote) Berzelius, J. J., method of, or fluorine...... 185

Bibliography of rock analysis............ 13

Bisbee, H., Richards, T. W., and McCaffrey,

C. T., cited................ 119

Blair, A. A., crucible tongs of............ 30 Blast. temperature of, test of....... 95 (footnote) Bloor, W. R., cited.................... 97 Blount, Bertram, cited.............. 94, 215 Blum, William, cited . . . . . . . . . . . ...... 192 Bolm, F., cited............... 173 (footnote) Bolton, H. C., cited ..................... 210 Boric oxide, fusion of . ................ 86 Boric oxide reagent, preparation of ........ 86 Boron, determination of ............... 199-200 occurrence of, in rocks............... 27 Bornemann, E., cited............74 (footnote) Bravo, José J., cited................. $\quad 25$ Bray, W. C., cited..................... 38 Broockmann, K., cited............... 126 Browning, P. E., cited ................. 120 Brühl, E., and Friedheim, C., cited..... 115,147 Brunck, O., cited................... 121,123 basic-acetate method of, in separation of iron, manganese, etc. 101-102 (footnote)

Brush, G. J., method of, in water determination

Buckley, E. R., method of, for determination of porosity............. 45-46 Bunsen, R., cited.........65,91,174 (footnote) 
Calcite, tests for ................ 179, 210-211 Calcium, determination of . 118-119, 121-123, 221, 229 ignition of oxalate of. precipitability of, by ammonium persulphate....................... rèmoval of, from magnesium pyrophosphate...................... 126-127

in alkali determination............ 173 separation of strontium from.......... 119-120 Calcium chloride reagent, deterioration of ... 74 Calcium fluoride, testing of precipitate of.. 186-187 Calcium oxalate, solubility of.............. $\quad 119$ Cameron, A., cited ..................... 91 Campbell, E. D., and Hess, W. H., cited.... 112 Carbon, determination of ............ 182,224-225 effect of, on ferrous-iron determination... 165, $166,222,224$ occurrence of, in rocks $165.182,209$

Carbonate rocks, analysis of analysis of defects of, as usually made . . 206-207 condensed forms of . . . . . . . . . . 228-232

detailed methods of.............. 212-228 causes of color of .................... 208 ignition loss of ...................., 231-232 importance of........................ 14 impurities in ....................... 209 methods of decomposing......... 212-214,228 mineral composition of............... 207-209 qualitative comparison of silicate rocks and $\ldots . . . . . . . . . . . . . . . . .205$ qualitative tests of.................. 209-211

Carbonates, qualitative tests of.......... 209-211 Carbon dioxide, direct determination of...... 179-

direct determination of, apparatus for, $181,182,224$ figure showing............... 180 expulsion of, by heat............... 200-201 indirect determination of ............ 181-182 Carbon monoxide, evolution of, on heating rocks...................... 201

Carnot, A., cited....................... 110

Cathrein, A., cited.... 111 (footnote), 135 (footnote)

Cerium, detection of.

occurrence of, in piedmontite... 100 (footnote)

Chalcopyrite, occurrence of, in rocks........ 26

Chapin, W. H., cited.................... 200

Chatard, T. M., cited................135, 145-146 drying oven of, description and figure of.. 76-77

Chlorine, condition of, in rocks............. 182 determination of................ 183-184, 225 occurrence of, in rocks................. 26

Chromium, colorimetric determination of.... 138$139,147-148,150-154,220$ colorimetric determination of, comparison of gravimetric method and... 148

effect of vanadium on................ 146 gravimetric determination of............ 146 occurrence of, in rocks................. 24,154

Citricacid, relative solubility of carbonates in. 210

Claasen, Edo, cited..................... 150

Clarke, F. W., cited ..................... 20

Classen, A., cited......................... 40

Cloedt, E. v., and Jannasch, P., cited...... 115,147

Cobalt, detection of....................... 115 determination of................. 116,220 occurrence of , in rocks................. 25

Coblentz, W. W., cited.................. 63
Colorimeters, description of and figures showing..................... 33-38

Colorimeteric methods, for determination of chromium................. 147-148

for determination of chromium, comparison of gravimetric method and $\ldots \ldots \ldots \ldots \ldots \ldots \ldots \ldots, 148$

for determination of manganese . . . ... 116-118 of titanium..................... 128-134 of vanadium................... 154 Columbium, occurrence of, in rocks......... $\quad 27$ Contamination of sample by agate.......... 56 by steel............................. 51

Cooke, J. P., method of, for determination of ferrous iron.......... 161, 168-170 method of, apparatus for, figure showing. $\quad 170$ Coppadoro, A., cited............. 108 (footnote) Copper, determination of ................116, 220

occurrence of, in rocks................ 25

Cramer, E., cited ....................... $\quad 46$ Cross, W., and others, cited.............. . 42 Crucible; disk for, description and figure of . . 22-23

tongs for, description and figure of...... 30 Crucibles, platinum, loss of weight of ........ 94-95 (footnote), 105 (footnote)

Crushing, methods of ................... 50-52 Crystal water. See Water.

Curtis, J. S., cited ........................ 201

Czudnowitz, C., cited................... 149

D.

Daniel, K., cited. ............... 184 (footnote)

Davis, J. F., cited....................... 142

Davy, Humphrey, cited . .......... 86 (footnote)

Day, A. L., and Allen, E. T., cited....... 48-49, 60 pycnometer method of . .............. 48-49

Delesse, A., cited...................... 202

Desiccators, use of .................. $68,69,70$

Determinations, importance of order of..... 41-42 Deussen, E., cited ................ 105 (footnote), 161 (footnote), 162

Dieulafait, L., cited.......... 20, 22 (footnote), 201 Dittrich, Max, cited......... 13, 28, 65, 83, 88, 100 ,

$105,112,138,143$ (footnote), 176

Dittrich, Max, and Freund, S., methods of, in separation of titanium from iron .................. 112, 136-138

methods of, in separation of zirconium from iron......... 138 (footnote), 142

Dolomite, definition of . . . . . . . . . . . . . . . 206

tests for ........................ 211

Drying before analysis, uselessness of ...... 65-66 Drying for hygroscopic tests, process of . . . . 67-70 Dunn, J. T., and Pattinson, I., cited. . . ... 195 Dunnington, F. P., cited................ 129 Duschak, L. H., and Hulett, G. A., cited... 194, E. 196,197

Eakins, L. G., cited................... 47-48 Eddy, E. A., and Gooch, F. A., cited...... 176 Electrification of glass tubes, influence of, on weight. .................. 74

Ellis, C. W. H., mortar of, description and figure of

Erdmann, H., cited..................... 202

Error in analysis, allowable limits of....... 28 
Errors due to abrasion of crushing and grind. ing implements . . . . . . . . . 51,56 grinding of sample...... 52-54,64-65, 154-157 variation of blast temperature... 95 (footnote)

\section{F.}

Faber, P., cited.................... 129,134

Feldspars, error in calculation of.......... $\quad 20$

Ferric iron, calculation of ............... 110 See also Iron.

Ferrous iron, determination of . . . 154-171, 222-224 determination of, hydrofluoric acid method of ................. 161-171

sealed tube method of .......... 157-161

oxidation of, by grinding.......... 53, 154-157

by free oxygen in presence of hydro-

fluoric acid or a fluoride......... 163

preparation of sample for determination of...................... 157

See also Iron.

Filter paper, macerated, use of, for precipitations.

Fischer, T., cited...................... 153

Fluid inclusions, volatilization of......... 201

Fluorine, determination of.......... 184-193, 226 influence of, in aluminum determination................. 99 (footnote)

in silica determination.... 84,86 (footnote) occurrence of, in rocks................ 26-27 qualitative tests for................ 184

Folin, Otto, cited............ 194 (footnote), 195

Foote, H. W. and Penfield, S. L., cited .... 200

Forchhamuner, J. G., cited................. 201

Ford, W. E., cited ..................... 200

Foullon, H. von, cited................. 201

Franz, B., and Streit, G., cited........... 142

Fresenius, R., cited.............. 119 (footnote)

Freund, S., and Dittrich, Max., methods of, in separation of titanium from iron.................112,136-138

zirconium from iron....... 138 (footnote), 142 Friedel, G., cited....................... 69, 70

Friedheim, C., and Brühl, E.,cited...... 115, 147

Friedheim, C., and Pinagel, A., cited..... 92

Funk, W., basic-acetate method of, for separation of iron, manganese, etc.............. 101-102 (footnote)

G.

Gage, R. B., cited............... 163 (footnote)

Galena, occurrence of, in carbonate rocks.... 209

Gases, evolution of, on heating rocks . . . . . 201-203

Gautier, A., cited ..................... 202

Gibbs, Wolcott, cited................... 124

Gilbert, J. P., cited . . . . . . . . . . . . . 92, 215

Glassware, inferiority of ................. $\quad 39$

Glucinum, occurrence of, in rocks.......... 27

Gold, occurrence of, in rocks . . ........... $\quad 27$

Gooch, F. A., apparatus of, for water determination, description and figures of ..................... $78-81$ cited.................. 111,199 (footnote) method of, in determination of lithium. . 175 In determination of titanium..... 134-136 Gooch, F.A., and Austin, M., cited. 116 (footnote), $123-124,145$

Gooch, F. A., and Eddy, E. A.. cited....... 176
Page.

Gooch, F. A., and Jones, L.C., cited.. 199 (footnote) Gooch, F. A., and Newton, H. D., cited .... 109

(footnote)

Grinder, mechanical...................... 55

Grinding, effect of, on composition of sam-

ple............... 52-54,64,154-157

methods of ....................... $52-56$

\section{$\mathrm{H}$.}

Hall, R. W., cited ............... 94 (footnote)

Hamberg, A., cited..................... $\quad 62$

Harper, D. N., and Penfield, S. L., cited.... 99

Harrington, B. J., cited.................... 209

Harrison, J. B., cited.................. 25, 27

Hauynite, evidence as to presence of........ 197 occurrence of, in rocks................ 26

Hayes, A. A., cited..................... 20

Heidenreich, O., and Jannasch, P., boricoxide method of, for decomposing silicates.................... 86

Hempel, w., cited...................... 56

Hewett, Foster, cited................... 25

Hess, W. H., and Campbell, E. D., cited.... 112

Hinden, F., cited..................... 211

Hinrichsen, F. W., cited............... 84, 99

Hintz, E., and Weber, H., cited.... 194 (footnote),

195,196

Elolthof, C., cited...................... 88,90

Horn, D. W., cited .............. 148 (footnote)

Hulett, G. A., and Berger, C. W., cited..... 94

(footnote)

Hulett, G. A., and Duschek, L. H., cited.... 194,

196,197

Hutchinson, A., cited ................... 210

Hüttner, K., cited...................... 202

Hydrofluoric acid reagent, quality of ......... 39

Fydrofluoric acid method of determining

ferrous iron............... 161-171

Hydrogen, evolution of, on heating rocks.... 201 discriminating between conditions of, in

minerals................... 58-63

rôle of; in minerals................. $57-63$

system of classification of, in minerals... 63

Hydrogen peroxide reagent, cuality of...... 40

Hydrogen sulphide, as impurity in carbon dioxide.............. 108 (footnote)

occurrence of, in carbonate rocks........ 209

Hygroscopic water, determination of...... $67-70$,

73-76, 227

See alsn Water.

I.

Iddings, J. P., and others, cited ........... 42

Ignition, loss by carbonate rocks on ...... 231-232

loss on, inaccuracy of water determina-

tion by ................... 71

temperature of, variations in..... 95 (footnote)

Iron, correction of titanium determination

for ...................... 132-134

determination of ................. 107-110,

154-171, 215-218, 222-223, 229

effect of carbonaceous matter on ..... 165 ,

$166,222,223$

effect of sulphides on.. 158-159, 165-166, 199

effect of vanadium on......... 109-110, 166 ignition of precipitate of oxide of...... 104-105 occurrence of, in carbonate rocks..... 207-208 
Iron, oxidizability of divalent, by free oxygen,

in presence of a fluoride........ 163 precipitation of........ 99-103, 216-217, 229-230 recovery of, from filtrate............ 103-104 reduction of ....................... 107-108

separation of, from aluminum ......... 111-113

in titanium determination......... 134-135

solution of oxide of, by alkali fusion..... 111 titration of ........ 108-110, 167-170, 218, 222-223 See also Ferric iron; Ferrous iron.

Iron oxides, error in determination of....... 21, $110,154-157,162-163,165-166.171$

\section{J.}

Jannasch, P., cited . 102 (footnote) nickel-becher of .................... 31 silicate decomposition methods of...... $85-86$ water determinations of, apparatus for, figure of................... $82-83$

method of ...................... 84 work of .......................... 84

Jannasch, P., and Cloedt, E. v., cited . .... 115, 147 Jannasch $P$., and Heidenreich, O., boricoxide method of ............... 86

Jannasch, P., and Weber H., cited.......... 86-87 Jannettaz, E., cited .................... 210 Järvinen, K. K., cited.................. 124, 126 Jones, L. C., and Gooch, F. A., cited. 199 (footnote) Johnston, J., and Allen, E. T., cited. 194 (footnote), $195,196,197$

Jordis, E., and Kanter, E. H., cited... 95 (footnote) Jordis, E., and Ludewig, W., cited... 93 (footnote)

\section{K.}

Kanter, E. H., and Jordis, E., cited... 95 (footnote) Kobell, F, von, cited .................... 59 Knop, A., cited................. 107 (footnote) Koch, A. A., and Tread well, F. P., cited.. 186, 187 Koninck, L. L. de, cited.............. 126, 158 Koningh, L. de, cited............. 147 (footnote) Kortright, F. L., cited.................... 93 Krauch, C., cited........................ $\quad 39$

Kreider, J. L., apparatus of, for determination of volatile substances, description and figure of. 181-182

L.

Lang, W. R., and Manning, R. J., cited .... 200 (footnote)

Lazurite, evidence of presence of, in rocks... 197 occurrence of, in rocks................ 26

Lead, determination of................... 220 occurrence of, in rocks.............. 27, 208

Le Chatelier, H., cited ................... $\quad 57$

Lemberg, J., cited...................... 211

Limestones, causes of color in............. 208 minor constituents of ................. 207

Limestones, dolomitic, definition of ......... 206

Limestones, magnesian, definition of....... 206

Lindemann, O., cited.................. 149

Lindo, D., cited ......................... 92

Literature of analysis..................... 13

Lithium, determination of.............. 174-175 occurrence of, in rocks.............. 26

Low, W. H., cited............... 200 (footnote)

Löwe, J., clay disks of ........................
Page.

Löwenstein, E., cited................... 57, 62 Ludewig, W., and Jordis, E., cited.......... 93 Ludwig, E., cited....................... 91 Luedeking, C., and Wheeler, H. A., cited.... 202 Lunge, G., cited..................... 33, 204 Lunge, G., and Millberg, C., cited........ 95, 204 Lunge, G., and Stierlin, R., cited .......... 194

(footnote), 195

- M.

Macerated filter paper, use of, for precipitatation.................... 103,104

McBride, R. S., cited.............. 108 (footnote)

McCaffrey, C. T., Bisbee, H., and Richards, T. W., cited..................

McKenna Brothers Brass Co., ore grinder of, description and figure of..... $55-56$

Magnesium, determination of. . 123-126, 221, 229-230 occurrence of, in carbonate rocks........ 206 precipitate of contaminants of, removal of ...................... 126-128

ignition of $\ldots \ldots \ldots \ldots \ldots \ldots \ldots \ldots \ldots \ldots, 120,127$ precipitation of . . . . . . . . . . . . . . . . 124-125 essentials of ................. 123-124 separation of, in alkali determination.. 176-177 Maillard, L., cited....................... 154 Manasse, O., cited .............. 151 (footnote) Manganese, determination of........... 113-118, $127-128,219-220,221,230$ occurrence of, in rocks............. 25, 207 oxidizability of divalent, in presence of hydrofluoric acid . . . . . . . . . . . 162-163 precipitation of . ..... 102-103, 114-115, 216,217 removal of, from magnesium pyrophosphate.................. 127-128

Manning, R. J., and Lang, W. R., cited, 200 (footnote)

Mar, F: W., cited...................... 140 Marble, contamination of, by sulphides,

108 (footnote)

Mauzelius, R., cited.......... 53,64 (footnote), 154 (footnote)

Meade, R. K., cited............. 214 (footnote)

Mechanical separation, literature of......... 14

Meigen, $W$., cited..................... 210

Meineke, C., cited....................... 91

Merwin, H. E., cited................ 129, 130, 134 method of, for fluorine............... 191-193

Meteorites, analysis of.................. 14

Microscopic examination, value of........ 17-18

Millberg, C., and Lunge, G., cited........ . 95, 204

Minerals, specific gravity of fragments of.... 46-47 Mingaye, J. C. H., cited................... 21

Minor, J. C., and Penfield, S. L., cited....... 186 Mitchell, W. L., and Wells, H. L., cited, 108 (footnote)

Mitscherlich method of ferrous iron deter- mination............... 157, 159-161 objections to.......... 157, 158, 159 (footnote) Mittasch, A. , basic acetate method of, for separation of manganese..... 101 (footnote)

Moissan, H., cited.............. 200 (footnote) Molybdenum, determination of....... 150-151, 220 occurrence of. in rocks......... 21,27, 149, 209

Moore, G. E., device of, to remove air from mineral powders, description and figure of . ................. 48-49 
Page.

Morgan, G. T., cited

182,225

Morley, E. W., cited

Morosewicz, J., cited. 79,172 (footnote)

Mortar, for crushing. 174 (footnote)

sloureu, C., cited. 202 (footnote)

\section{$\mathrm{N}$.}

Nepheline, detection of, in presence of olivine. 203 Neubauer, H., cited............... 123-124, 125, 126 Neustadl, L., and Skrabal, A., cited........ 120 Newton, H. D., cited............... 109 (footnote) Newton, H. D., and Gooch, F. A., cited, 109 (footnote)

Nickel, determination of............... 116, 220 occurrence of, in rocks................ 25 precipitation of .................... 114-115

Nickel-becher, description of.............. 31

Nitrogen, occurrence of, in rocks........... 202

Noselite, evidence as to presence of.......... 197 occurrence of, in rocks................. $\quad 26$

Noyes, A. A., and others, cited............ 38

Noyes, W. A., cited........................ 133

\section{0.}

Organic matter, removal of ...............

Oxidation, incompleteness of, on ignition of ferrous compounds.

$$
P \text {. }
$$

Panebianco, G., cited...

Pattinson, $\mathrm{T}$, and Dunn, $\mathrm{T}$ T , cited.....

Penfield, S. L., cited............. 46,79 (footnote) method of, for water determination..... 72,

apparatus for, figures showing. . . $72,73,75$

Penfield, S. L., and Foote, H. W., cited...... 200 Penfield, S. L., and Harper, D. N., cited...... 99 Penfield, S. L., and Minor, J. C., cited....... 186 Penfield, S. L., and Sperry, E. S., cited ..... 199

(footnote)

Peters, R., cited. 163 Pfaft, F. W., cited...................... 206

Phenylhydrazine reagent, contamination of. 113 (footnote)

Phosphoric acid, decolorant for ferric solutions....................... 134 effect of, on color of titanium peroxide.... 129

Phosphorus, determination of...... 144-146, 222, 230 loss of by volatization ................ 111 occurrence of in rocks.............. 26,209 precipitation of........... 99-103, 145, 216-217

Phosphorus salt reagent, quality of........ 40 Piedmontite, rare earths in......... 100 (footnote) Pinagel, A., and Friedheim, C., cited........ 92 Pirsson, L. V., cited .................... 29,203 Pirsson, I. V., and others, cited............. 42 Platinum in filtrates .................... 97

Platinum, occurrence of, in rocks........... 27 precipitation of ........... 98, 107 (footnote) reduction of, from solution....... 174 (footnote)

Platinum crucibles, loss of weight in........ 9495 (footnote), 105 (footnote)

Platinum ware, iron in ......... 41,95 (footnote) Porosity, determination of .............43, 45-46

Potassium, determination of ....... 173-174,177-178 as chlorplatinate.................. 174,178 as perchlorate...................... 178 separation of, by sodium cobaltinitrite. 177-178
Potassium bisulphate reagent, conversion of, to pyrosulphate............ 40

objections to use of ............ 105 (footnote) quality of ......................... 40

relative solubility of carbonates in.... 210

Potassium pyrosulphate reagent, advantages in use of ......... 105 (footnote)

Powell, N. S., and Richards, J. W., cited..... 210 Pratt, J. H., method of, in ferrous iron determination........... 164-165, 167-168 Precht, H., cited............... 174 (footnote) Pycnometer method of specific gravity determinations................... 47-49

Pyrite, effect of, on ferrous iron determinetion....................... 165-166

occurrence of, in rocks................ 26, 209 Pyrrhotite, evidence as to presence of, in rocks.................. 26, 197,199

R.

Radiators for evaporating solutions........ 31-32 Raffa, E., cited....................... 124 Ransome, F.L.,cited.. 24 (footnote), 149 (footnote) Rare earths, caution against overlooking of ...............100 (footnote), 114 determination of........... 138-139, 143-144, 220 occurrence of, in rocks. . . . . . . . . . . 23,27 Reagents, quality of .................... $39-41$

testing of........................... 40-41

Richards, J. W., and Powell, N. S., cited.... 210 Richards, T. W., McCaffrey, C. 'T., and Bisbee, H., cited................ 119 Ridsdale, C. F., cited................ 110,152 Ries, H., cited....................... 206 Riggs, R. B., method of, for separation of magnesium from alkalies....... 177

Robertson, J. D., cited ................. 201 Rose, II., cited... 111 (footnote), 185 (footnote), 202 Rosenbladt, Th., cited............ 199 (footnote)

\section{$\mathrm{S}$}

Sample, contamination of, by agate....... 56 contamination of, by steel....... 51 (footnote) fineness of ........................ 52,53 preparation of ................... 50-56,157 weight of ....................... 56 Sandberger, F., cited........... 22 (footnote), 201 Sargent, G. W., cited................... 200 Schaffgotsch's solution, character of. 176 (footnote) use of . . ............. 176-177, 185 (footnote) Schaller, W. T., cited............. 173 (footnote) Schreiner, Oswald, colorimeter of, descrip-

tion and figure of.............. 37-38

Scoop. Sec Weighing, scoop for.

Seemann, F., cited.............. 185 (footnote), 187 Seger, H., cited........................ 46 Separation, mechanical, literature of........ 14 Shimer, P. W., cited.............. 214 (footnote) Sieves, metal, unavailability of............. 51 Silica, adherence of, to dish............... 93 dehydration of....................... 91-94' determination of .................... 83-97 $105-107,188,206-214,218,229$

accuracy of ................ 96,106-107 impurities in, correction for............ 95-96 precipitate of, ignition of............. 94-95 recovery of, from alumina precipitate.. 105-107 
Silica, removal of in fluorine determination T'age. in sulphur determination.......... 198 residue from, composition of . . . . . . . . 96-97 separation of, from alumina, etc...... . 83-97,

$214-215,230$

fluxes used in .. $84-90$

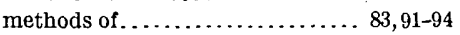

Silica, soluble, determination of . . . . . . . . 203-204

Silicate rocks, analysis of ............... 16-204

analysis of, methods of . . . . . . . . . . . . 41-204

constituents in. . . . . . . . . . . . . . . . 22-27

decomposition of, by hydrofluoric and sulphuric acids........... 83-84, 131

literature of $\ldots \ldots \ldots \ldots \ldots \ldots \ldots \ldots \ldots .13$

methods of decomposition of.......... 83-90

Siliceous limestones, character of . . . ...... 208

Silver, occurrence of, in rocks............ 27

Silvestri, O., cited....................... 203

Sjollema, B., cited........................ 204

Skrebal, A., and Neustadl, L., cited....... 120

Smith, J. Lawrence, cited . . . . . . . 105 (footnote) method of, for alkali determination... 171-173 apparatus for, figure showing...... 172

Sodium, determination of . . . . . . . . . . . . 174-175

Sodium bisulphate, objections to.... 105 (footnote) quality of

Sodium carbonate fusion . . . . . . . . . . . $87-90$

Sodium carbonate melt, effect of leaching of, with water................ 139

Sodium carbonate reagent, quality of ..... 40-41, 88 cobaltinitrite, reagent.............. 177

Sodium hydroxide reagent, quality. of ...... 41

Sodium potassium carbonate, comparison of sodium carbonate and ........ $87-88$

Sodium pyrosulphate reagent, preparation

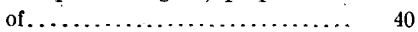
use of, advantages............ 105 (footnote)

Solutions, heavy, unavailability of, in specific gravity determinations of rocks.

Sonstadt, E., cited ............. 174 (footnote)

Spear, F. B., cited.................. 38

Specific gravity, determination of ......... 43-50

Sperry, E. S., and Penfield, S. L., cited..... 199

(footnote)

Sphalerite, occurrence of, in carbonate rocks.. 209 Steiger, George, cited .......... 114 (footnote), $129,163,173$ (footnote) calorimeter of, description and figures of . 35-37 drying apparatus of, description and fig -

ures of . . . . . . . . . . . . $75-76$ method of, for fluorine.............. 188-190

Steinlen, R. L., cited ............ 172 (footnote) Stierlin, R., and Lunge, G., cited ... 194 (footnote),

Stoddart, C. W., cited............ 198 (footnote) Stokes, H. N., cited.... 141,158, 159 (footnote), 165 Streit, G., and Franz, B., cited............ 142 Strontium, determination of......... 119-120,221 occurrence of, in rocks............. 20, 25 separation of, from calcium .......... 119-120

from magnesium.............. 118-119

Strontium sulphate, spectroscopic testing of .. $\quad 140$ Sulphides, conversion of, to sulphates in limestones................. 227 influence of, on determination of ferrous Iron. $158,165-166$
Page.

Sulphur, condition of, determination of ....... 197-

198,226

determination of...... 138-139, 198-199, 226-227 errors in determination of............ 193-197 failure to determine, error arising from ... 21 occurrence of, in rocks................ 21,26 removal of, in alkali determination....... 173 Sulphur dioxide, reagent; quality of. 151 (footnote)

\section{T.}

Tammann, G., cited.................... 57,62

Tantalum, occurrence of, in rocks........... 27

Tartaric acid, relative solubility of carbonates in......................... 210

removal of, in titanium determination... 135

Thörner, T. W., cited..................... 46 Thorpe, T. E., cited..................... 20 Thugutt, St. J., cited ............ 59 (footnotè) Tin, occurrence of, in rocks............... 27 Titanic cxide, solubility of, in alkalies .... 111-112 Titanium, colorimetric determination of ..... 128134,219

colorimetric determination of, superiority of ..................... 138 gravimetric determination of. . 111, 134-138, 230 loss of ....................... 131 (footnote) occurrence of, in rocks.............. 23-24 precipitation of.. 98, 107 (footnote), 112, 216-217 Titanium sulphate reagent, preparation of .. 130 T'oluene bath, description and use of...... 75-76,79 Trace, definition of ....................... 29 Travers, M. W., cited ................... 202 Tread well, F. P., cited . ................ 95, 185 method of, for ferrous iron determination................... 161,170-171

apparatus for, figure showing....... 170 Treadwell, F. P., and Koch., A. A., cited . 186, 187 Tschermak, G., cited .................... 57 Tungsten, occurrence of, in rocks........... 27 Tyrolite, drying of, experiment on........... 68,69 U.

Ulbricht, R., cited. 126 Ullmann, C., cited .............. 160 (footnote) Uranium, occurrence of, in rocks.......... 27

V.

Vanadium, colorimetric determination of .... 154 effect of, on iron determination. 109-110, 149, 166 failure to determine, error from ......... 21 gravimetric determination of . . 150-154, 220-221 occurrence of, in rocks.......... 20,24, 149, 209 Veitch, F. P., cited..................... 84,99 Vogt, J. H. L., cited................... 158 Volatile substances, determination of, apparatus for, description and fig. ure of...................... 181 Volhard, J., cited................ 116 (footnote) w.

Walters, H. E., cited............... 117 (footnote) Walton, J. H., jr., cited . . ................ 134 Warth, H., cited ................ 71, (footnote) Washington, H. S., cited ........ 13, 17 (footnote), 18 (footnote), 22 (footnote), $23,25,28$, 29, 52,108 (footnote), 114 (footnote) 
Washington, H. S., method of, in determi-

Page. nation of phosphorus. 144-145

Washington, H. S.; and others, cited........ 42 Water, direct determination of... 72-83, 225, 227-228 direct determination of, apparatus for, description and figures of....... 72-83 discriminating between conditions of, in minerals.

indirect determination of .............. $67-71$

methods of determination of........... 67-83

rôle of, in minerals.................. 57-63

suspension in, determination of specific gravity by................. 43-46

system of classification of, in minerals... 63

Water lost by grinding........... 59 (footnote), 64 taken up by grinding... 53,59 (footnote), 64,65

Weber, H., and Hintz, E., cited.... 194 (footnote),

Weber, H., and Jannasch, P.; boric oxide, method of, for decomposing silicates..................... 86,87

Weighing scoop, description and figure of....

Weller, A., method of, for determination of titanium.
Wells, $\mathrm{H}, \mathrm{A}$, and Mitchell; W. $\mathrm{L}$, citech....

(footnote)

Wells, R. C., cited ................. 105 (footnote)

Wheeler, H. A., and Luedeking, C., cited.... 202

Wherry, E. T., cited.................... 200

Williamson; J. A., cited .................. 39 Wülfing, E. A., cited........... 158, 176 (footnote)

\section{Z.}

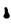

Zambonini, F., cited.............. 57,60,61, 62 Zeolitic minerals, dehydration of..... $60-61,69-70$ occurrence of, in rocks.......... 44 (footnote)

Zimmerman, C., method of, for separation of magnesia.

Zinc, determination of................. 115, 220 occurrence of, in rocks............... 27, 208 precipitation of .................... 114-116

Zinc oxide solution, preparation and use of.. 185 Zirconium, determination of............ 111-112, $137,138-139,140-142$ difficulty with, in gravimetric titanlum determination............... 130

128 occurrence of, in rocks................. 24 